\title{
Engineering Secure Two-Party Computation Protocols
}

Advances in Design, Optimization, and Applications of Efficient Secure Function Evaluation

\section{Thomas Schneider}

\begin{abstract}
Dissertation
zur Erlangung des Grades eines Doktor-Ingenieurs der Fakultät für Elektrotechnik und Informationstechnik an der Ruhr-Universität Bochum.
\end{abstract}

\section{PhD Committee:}

Prof. Dr.-Ing. Thomas Musch (chair), Prof. Dr. Benny Pinkas ( $2^{\text {nd }}$ supervisor), Prof. Dr.-Ing. Ahmad-Reza Sadeghi ( $1^{\text {st }}$ supervisor), Prof. Dr. Jörg Schwenk, Prof. Dr.-Ing. York Tüchelmann

Bochum, February 9

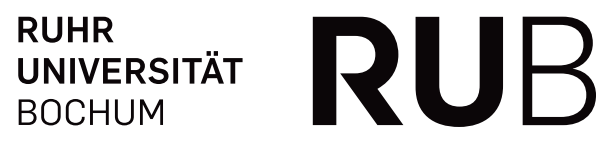





\section{Summary}

Secure two-party computation, called Secure Function Evaluation (SFE), enables two mutually mistrusting parties (client \& server) to evaluate an arbitrary function $f$ on their respective private inputs $x, y$ while revealing nothing but the result $z=f(x, y)$. Although such generic techniques were widely believed to be inefficient, the rapidly growing speed of computers and communication networks, algorithmic improvements, automatic generation and optimizations of SFE protocols have made them usable in practical application scenarios.

This thesis summarizes the state of the art in efficient techniques for SFE and presents the following advances in the design, optimization and applications of efficient SFE protocols.

Circuit Optimizations and Constructions. The complexity of today's most efficient SFE protocols depends linearly on the size of the boolean circuit representation of the evaluated function. Further, recent techniques for SFE based on improved Garbled Circuits allow for very efficient secure evaluation of XOR gates.

We give transformations that substantially reduce the size of boolean circuits if the costs for evaluating XOR gates are lower than for other types of gates. Our optimizations provide more efficient circuits for standard functionalities such as integer comparison and fast multiplication.

Applications that benefit from our improvements are secure first-price auctions.

Parts of these results are published in:

- Section 3 of Benny Pinkas, Thomas Schneider, Nigel P. Smart, Stephen C. Williams. Secure Two-Party Computation is Practical. In $15^{\text {th }}$ Advances in Cryptology - ASIACRYPT'09.

- Annika Paus, Ahmad-Reza Sadeghi, Thomas Schneider. Practical Secure Evaluation of SemiPrivate Functions. In $7^{\text {th }}$ International Conference on Applied Cryptography and Network Security (ACNS'O9).

- Vladimir Kolesnikov, Ahmad-Reza Sadeghi, Thomas Schneider. Improved Garbled Circuit Building Blocks and Applications to Auctions and Computing Minima. In $8^{\text {th }}$ International Conference on Cryptology And Network Security (CANS'09).

Hardware-Assisted Garbled Circuit Protocols. We improve the deployability of SFE protocols by using tamper-proof hardware (HW) tokens.

In particular, Garbled Circuits (GCs) can be generated by a tamper-proof HW token which is provided by the server to a client but not trusted by the client. The 
presented $\mathrm{HW}$-assisted SFE protocol makes the communication between client and server independent of the size of the evaluated function. Further, we show how GCs can be evaluated in $\mathrm{HW}$ in a leakage resilient way, so-called One-Time Programs

As application we show how the combination of GCs and tamper-proof $\mathrm{HW}$ allows to securely outsource data to an untrusted cloud service provider such that arbitrary functions can be computed securely on the data with low latency.

Parts of these results are published in:

- Kimmo Järvinen, Vladimir Kolesnikov, Ahmad-Reza Sadeghi, Thomas Schneider. Embedded SFE: Offloading Server and Network using Hardware Tokens. In $14^{\text {th }}$ International Conference on Financial Cryptography and Data Security (FC'10).

- Kimmo Järvinen, Vladimir Kolesnikov, Ahmad-Reza Sadeghi, Thomas Schneider. Garbled Circuits for Leakage-Resilience: Hardware Implementation and Evaluation of One-Time Programs. In 12 ${ }^{\text {th }}$ International Workshop on Cryptographic Hardware and Embedded Systems (CHES'10).

- Ahmad-Reza Sadeghi, Thomas Schneider, Marcel Winandy. Token-based Cloud Computing Secure Outsourcing of Data and Arbitrary Computations with Lower Latency. In $3^{\text {rd }}$ International Conference on Trust and Trustworthy Computing (TRUST'10) - Workshop on Trust in the Cloud.

Modular Design of Efficient SFE Protocols. Automatic generation of SFE protocols from high-level specifications makes SFE usable for application programmers and yields less error-prone implementations.

We present a framework which enables to modularly design efficient SFE protocols as sequence of operations on encrypted data. In our framework, efficient SFE protocols based on Homomorphic Encryption and Garbled Circuits can be combined while abstracting from the underlying cryptographic details. Our corresponding language and tool, called Tool for Automating Secure Two-partY computations (TASTY), allow to describe, automatically generate, execute, and benchmark such modular and efficient SFE protocols.

As application we show efficient protocols for privacy-preserving face recognition.

Parts of these results are published in:

- Wilko Henecka, Stefan Kögl, Ahmad-Reza Sadeghi, Thomas Schneider, Immo Wehrenberg. TASTY: Tool for Automating Secure Two-partY computations. In $17^{\text {th }}$ ACM Conference on Computer and Communications Security (CCS'10).

- Mauro Barni, Pierluigi Failla, Vladimir Kolesnikov, Riccardo Lazzeretti, Ahmad-Reza Sadeghi, Thomas Schneider. Secure Evaluation of Private Linear Branching Programs with Medical Applications. In $14^{\text {th }}$ European Symposium on Research in Computer Security (ESORICS'09).

- Ahmad-Reza Sadeghi, Thomas Schneider, Immo Wehrenberg. Efficient Privacy-Preserving Face Recognition. In $12^{\text {th }}$ International Conference on Information Security and Cryptology (ICISC'09). 


\section{Acknowledgements}

First and foremost I would like to thank my thesis advisors Ahmad-Reza Sadeghi and Benny Pinkas. I am thankful to Ahmad for his outstanding supervision and the opportunities he opened for me. His mentorship in form of a mixture of giving initial directions and topics to look into, the freedom to develop my own research ideas, and a lot of valuable feedback was just perfect. He showed me that our research community is like a big family and gave me lots of chances to talk to, learn from, work with, and spend a great time together with many outstanding fellow researchers. I am very honored to have Benny as external advisor and thank him a lot for our productive discussions on deep technical details and his hospitality.

Thanks also to all my co-authors beyond Ahmad and Benny with whom I had the pleasure to collaborate in different areas of cryptography and security: I learned a lot from experienced senior researchers in secure computation, efficient implementations, zero-knowledge, signal processing, and formal verification (Endre Bangerter, Manuel Barbosa, Mauro Barni, Jan Camenisch, Marc Fischlin, Kimmo Järvinen, Vladimir Kolesnikov, Nigel P. Smart, Joe-Kai Tsay, Ivan Visconti). Thanks to my fellow Ph.D. students (José Bacelar Almeida, Stefania Barzan, Thomas Briner, Pierluigi Failla, Stephan Krenn, Riccardo Lazzeretti, Stephen C. Williams, Marcel Winandy) for the splendid exchange of mutually stimulating ideas we had. Special thanks also to the undergraduate students whom I was pleased to supervise and work with (Wilko Henecka, Stefan Kögl, Annika Paus, Immo Wehrenberg).

My research and travels were in part funded by EU projects Computer Aided Cryptography Engineering (CACE), Signal Processing in the EncryptEd Domain (SPEED), and the $2^{\text {nd }}$ European Network of Excellence in Cryptology (ECRYPT II). At the project meetings and other events I enjoyed a lot to meet, chat with, and get very helpful input from many other people, including Frederik Armknecht, Christian Cachin, Ivan Damgård, Juan A. Garay, Martin Geisler, Yuval Ishai, Jonathan Katz, Stefan Katzenbeisser, Yehuda Lindell, Mark Manulis, Jesper Buus Nielsen, Claudio Orlandi, Berry Schoenmakers, and Gene Tsudik.

Many thanks also to all my colleagues in the System Security Group and the Horst Görtz Institute for IT-Security in Bochum for their support and advice in all kinds of administrative issues and lots of insightful discussions during lunches and coffee breaks we enjoyed together.

Thanks as well to Wilko Henecka, Kimmo Järvinen, Berry Schoenmakers, and my family for proof-reading and commenting on drafts of this thesis.

Last but not least, I'd like to thank my girlfriend and future wife Karolin for all her support and understanding during the past three years in Bochum, our families and old friends for staying in touch, and those we made here for a great time.

Everything that can be counted does not necessarily count; everything that counts cannot necessarily be counted. (Albert Einstein) 


\section{Short Contents}

Summary

\begin{tabular}{|ll}
\hline Acknowledgements & iii
\end{tabular}

Structure iv

Short Contents . . . . . . . . . . . . . . . . . iv

Contents . . . . . . . . . . . . . . . . . $\mathrm{v}$

Lists . . . . . . . . . . . . . . . . . . . . ix

\begin{tabular}{lll}
\hline & Introduction & 1
\end{tabular}

1.1 Outline . . . . . . . . . . . . . . . . . . . . . . . . . . 2

1.2 Summary of Main Results . . . . . . . . . . . . . . . . . . . . . . . . . 3

2 Secure Function Evaluation Basics $\quad 5$

2.1 Common Notation and Definitions . . . . . . . . . . . . . . . . 5

$2.2 \quad$ Cryptographic Primitives for Secure Two-Party Computation . . . . . . 10

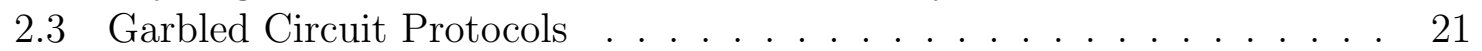

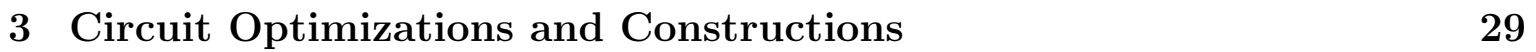

3.1 Motivation . . . . . . . . . . . . . . . . . . . . . . 29

$3.2 \quad$ Circuit Optimizations $\ldots \ldots \ldots \ldots$. . . . . . . . . . . . . . . . . . . 32

3.3 Efficient Circuit Constructions . . . . . . . . . . . . . . . . . . . . . . . . 39

3.4 Applications: Secure Comparison and Auctions . . . . . . . . . . . . 46

4 Hardware-Assisted Garbled Circuit Protocols 53

4.1 Creating Garbled Circuits with Hardware Token . . . . . . . . . . . . . 53

4.2 One-Time Programs $\ldots \ldots \ldots \ldots \ldots \ldots$. . . . . . . . . . . . . 64

$4.3 \quad$ Application: Privacy-Preserving Cloud Computing. . . . . . . . . . . . . 80

\begin{tabular}{|lll}
5 & Modular Design of Efficient SFE Protocols & 91
\end{tabular}

5.1 Framework for Modular SFE Protocols . . . . . . . . . . . . . . . . . . 92

5.2 Compiling Modular SFE Protocols . . . . . . . . . . . . . . . . . 96

5.3 Application: Privacy-Preserving Face Recognition . . . . . . . . . . . . 113

$\begin{array}{llr}6 & \text { Conclusion } & 129\end{array}$

6.1 Summary . . . . . . . . . . . . . . . . . . . . . . . . . . . 129

6.2 Directions for Future Research . . . . . . . . . . . . . . . . . . . . . . . 130

\begin{tabular}{lc}
\hline Bibliography & 133
\end{tabular}

\begin{tabular}{ll}
\hline About the Author & 149
\end{tabular} 


\section{Contents}

Summary i i

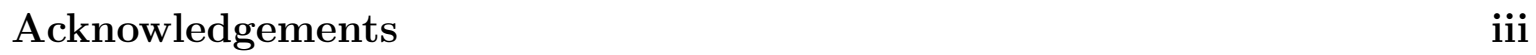

Structure iv

Short Contents . . . . . . . . . . . . . . . . . . iv

Contents . . . . . . . . . . . . . . . . . . . . . . v

Lists . . . . . . . . . . . . . . . . . . . . . . ix

List of Figures . . . . . . . . . . . . . . . . . . ix

List of Tables . . . . . . . . . . . . . . . . . . . . . . $\mathrm{x}$

List of Algorithms. . . . . . . . . . . . . . . . . . . . . $\mathrm{x}$

List of Acronyms . . . . . . . . . . . . . . . . . . . $\mathrm{x}$

$\begin{array}{lll}1 & \text { Introduction } & 1\end{array}$

1.1 Outline. . . . . . . . . . . . . . . . . . . . . . . . . . . 2

1.2 Summary of Main Results . . . . . . . . . . . . . . . . . . . . . . 3

2 Secure Function Evaluation Basics 5

2.1 Common Notation and Definitions . . . . . . . . . . . . . 5

2.1 .1 Notation . . . . . . . . . . . . . . . . . . 5

$2.1 .1 .1 \quad$ Basics $\ldots \ldots \ldots \ldots \ldots$

2.1.1.2 Security and Correctness Parameters . . . . . . . . 6

2.1 .2 Cryptographic Primitives . . . . . . . . . . . . . . 6

$2.1 .3 \quad$ Function Representations . . . . . . . . . . . . . . . . . . . . . . 7

2.1.3.1 Boolean Circuits . . . . . . . . . . . . . . . . . . . . 7

2.1.3.2 Arithmetic Circuits . . . . . . . . . . . . . . . . . 8

$2.1 .4 \quad$ Adversary Model . . . . . . . . . . . . . . . . . . . . . 8

2.1 .5 Random Oracle Model . . . . . . . . . . . . . . . . . . . . . . 9

$2.2 \quad$ Cryptographic Primitives for Secure Two-Party Computation . . . . . . 10

2.2.1 Homomorphic Encryption (HE) . . . . . . . . . . . . . . . 10

2.2.1.1 Additively Homomorphic Encryption . . . . . . . . . . 10

2.2.1.2 $\quad$ Fully Homomorphic Encryption . . . . . . . . . . . . . 11

2.2.1.3 Computing on Encrypted Data . . . . . . . . . . . . . 12

2.2 .2 Garbled Circuit (GC) Constructions . . . . . . . . . . . . 13

2.2.2.1 Components of GC Constructions . . . . . . . . . . . 13

2.2.2.2 Interfaces and Structure of GC Constructions . . . . . 15

$2.2 .2 .3 \quad$ Efficient GC Constructions . . . . . . . . . . . . . 16

2.2.2.4 Complexity of Efficient GC Constructions . . . . . . . 19

2.2 .3 Oblivious Transfer (OT) $\ldots \ldots \ldots \ldots$

2.2 .3 .1 Efficient OT Protocols . . . . . . . . . . . . 20 
$2.2 .3 .2 \quad$ Extending OT Efficiently $\ldots \ldots \ldots \ldots$

$2.2 .3 .3 \quad$ Pre-Computing OT. . . . . . . . . . . . . . . . . . . . . . . . 21

2.2 .3 .4 OT Complexity . . . . . . . . . . . . . . . . . 21

2.3 Garbled Circuit Protocols . . . . . . . . . . . . . . . . . . . . . . . . . 21

$2.3 .1 \quad$ Two-party Secure Function Evaluation (SFE) . . . . . . . . . . 22

2.3.1.1 SFE with Semi-honest Adversaries (Yao's Protocol)] . . 23

2.3 .1 .2 SFE with Stronger Adversaries . . . . . . . . . . 24

$2.3 .1 .3 \quad$ SFE with Private Functions . . . . . . . . . . . . 25

2.3 .2 Garbled Circuit Protocols with Multiple Parties . . . . . . . . . 25

2.3.2.1 Secure Multi-Party Computation with Two Servers . . 26

$2.3 .2 .2 \quad$ Secure Mobile Agents . . . . . . . . . . . 26

$\begin{array}{lll}3 & \text { Circuit Optimizations and Constructions } & 29\end{array}$

3.1 Motivation . . . . . . . . . . . . . . . . . . . . . . . . 29

3.1 .1 Protocols with Free XOR. . . . . . . . . . . . . . . . . . . . . 29

3.1 .2 Related Work . . . . . . . . . . . . . . . . . . . . 30

3.1 .3 Preliminaries and Notation . . . . . . . . . . . . . . . . . . . . . 31

3.2 Circuit Optimizations . . . . . . . . . . . . . . . . . . . . . . . . . . . 32

3.2 .1 Minimizing Circuits . . . . . . . . . . . . . . . . . . . . . . 32

3.2.1.1 $\quad$ Replacing Trivial Gates . . . . . . . . . . . . . . . . . 32

3.2 .1 .2 Removing Gates . . . . . . . . . . . . . . . . . . . . 34

3.2 .2 Minimizing Circuits with Free XOR . . . . . . . . . . . . . . 37

3.3 Efficient Circuit Constructions . . . . . . . . . . . . . . . . . . . . . . . 39

3.3 .1 Addition and Subtraction . . . . . . . . . . . . . . . . . . 39

3.3.1.1 $\quad$ Addition (ADD) . . . . . . . . . . . . . . . . . 39

$3.3 .1 .2 \quad$ Subtraction (SUB) $\ldots \ldots \ldots$. . . . . . . . . . . . . 40

3.3.1.3 Controlled Addition/Subtraction (ADDSUB) . . . . . . 40

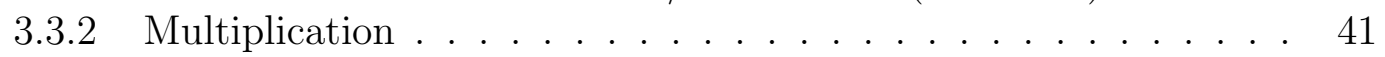

3.3.2.1 Textbook Multiplication . . . . . . . . . . . . . . . . . . . . . . . 41

3.3.2.2 $\quad$ Fast Multiplication . . . . . . . . . . . . . . . . . . . . 42

3.3.2.3 Multiplication Circuit Complexity . . . . . . . . . . . 43

3.3 .3 Comparison, Minima and Maxima . . . . . . . . . . . . . . . . . . . . 44

3.3.3.1 Comparison . . . . . . . . . . . . . . . . . . . . . . . . . . . 44

3.3.3.2 $\quad$ Multiplexer . . . . . . . . . . . . . . . . . . . . . . . 45

3.3.3.3 Minimum/Maximum Value and Index . . . . . . . . 45

3.4 Applications: Secure Comparison and Auctions . . . . . . . . . . . 46

3.4.1 Comparison (Millionaires Problem) . . . . . . . . . . . . . . . 46

3.4.1.1 Comparison with Pre-Computations . . . . . . . . . . 47

3.4.1.2 Complexity Evaluation . . . . . . . . . . . . . . . . . . . . . . . . . 47

3.4 .2 Auctions . . . . . . . . . . . . . . . . . . . . . . . . . . . . . . 49

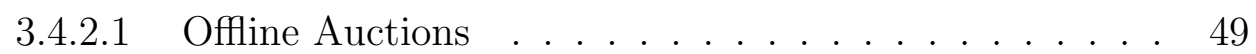

3.4 .2 .2 Online Auctions . . . . . . . . . . . . . . . 50 
4 Hardware-Assisted Garbled Circuit Protocols 53

$4.1 \quad$ Creating Garbled Circuits with Hardware Token . . . . . . . . . . . . . 53

$4.1 .1 \quad$ Motivation and Setting . . . . . . . . . . . . . . . . 54

$4.1 .2 \quad$ Related Work . . . . . . . . . . . . . . . . . . . . . . . 55

$4.1 .3 \quad$ Architecture, System and Trust Model . . . . . . . . . . . . . . 56

4.1 .4 Token-Assisted SFE $\ldots \ldots \ldots \ldots \ldots$

4.1 .4 .1 Protocols Overview . . . . . . . . . . . . . . . . . . . . 57

$4.1 .4 .2 \quad$ Circuit Representation . . . . . . . . . . . . . . 60

4.1.4.3 GC Creation with Stateful Token Using Secure Counter 61

4.1.4.4 GC Creation with Stateless Token (no Counter) . . . . 62

4.1.4.5 Hardware Implementation . . . . . . . . . . . . . . 63

4.2 One-Time Programs $\ldots \ldots \ldots \ldots \ldots$. . . . . . . . . . . . . . . . . . . . . . . . . . . . . . . 64

4.2 .1 Motivation $\ldots \ldots \ldots \ldots$. . . . . . . . . . . . . . . 64

4.2.1.1 Side-Channels and Protection . . . . . . . . . . . . . 64

4.2.1.2 SFE in Hardware and Leakage-Resilience. . . . . . . . 65

$4.2 .1 .3 \quad$ Our Objectives . . . . . . . . . . . . . . . . . 66

4.2 .2 Non-interactive GCs and One-Time Programs . . . . . . . . . . 66

4.2.2.1 $\quad$ Previous Works . . . . . . . . . . . . . . . . . 67

$4.2 .2 .2 \quad$ Extending One-Time Programs . . . . . . . . . . . . 68

$4.2 .2 .3 \quad$ Architecture for Using One-Time Programs . . . . . . 69

4.2.2.4 Fully Homomorphic Encryption against Leakage. . . . 72

$4.2 .3 \quad$ Evaluating GCs in HW . . . . . . . . . . . . . . . . . . . 72

4.2.3.1 GC Evaluation with Low Memory . . . . . . . . . . . 73

$4.2 .3 .2 \quad$ Architecture for Evaluating GCs in HW . . . . . . . . 73

4.2.3.3 Optimizations for Memory-Constrained Devices . . . . 76

$4.2 .3 .4 \quad$ Hardware Implementation . . . . . . . . . . . . . . . 77

4.3 Application: Privacy-Preserving Cloud Computing. . . . . . . . . . . . 80

4.3 .1 Motivation . . . . . . . . . . . . . . . 80

4.3 .2 Model for Privacy-Preserving Cloud Computing . . . . . . . . . 83

4.3.2.1 Tamper-Proof Hardware Token T . . . . . . . . . . . . 83

4.3 .2 .2 Preliminaries and Notation . . . . . . . . . . . . 84

$4.3 .3 \quad$ Architectures for Privacy-Preserving Cloud Computing . . . . . 85

4.3.3.1 $\quad$ Token Computes . . . . . . . . . . . . . . . . . 85

4.3 .3 .2 Cloud Computes . . . . . . . . . . . . . . . . . . . 86

$4.3 .3 .3 \quad$ Token Sets Up and Cloud Computes . . . . . . . . . . 87

4.3 .4 Performance Comparison . . . . . . . . . . . . . . . 88

\begin{tabular}{|lll}
5 & Modular Design of Efficient SFE Protocols & 91
\end{tabular}

5.1 Framework for Modular SFE Protocols . . . . . . . . . . . . . . . . . . 92

$5.1 .1 \quad$ Function Representations . . . . . . . . . . . . . . . . . . . . . . 92

5.1 .2 Modular SFE . . . . . . . . . . . . . . . . . . . . . . . . 93

5.1.2.1 Garbled Values and Conversions . . . . . . . . . . . . 93 
5.1.2.2 Homomorphic Values and Conversions . . . . . . . . . 94

5.1 .3 Conversion between Encrypted Values . . . . . . . . . . . . . . 95

5.1.3.1 Garbled Values to Homomorphic Values . . . . . . . . 95

5.1.3.2 Homomorphic Values to Garbled Values . . . . . . . . 96

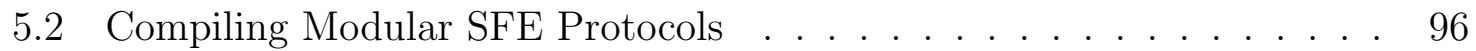

5.2 .1 Introduction . . . . . . . . . . . . . . . . . . . . . . 97

5.2 .1 .1 Motivation . . . . . . . . . . . . . . . . 97

5.2 .1 .2 Outline and Contribution . . . . . . . . . . . . . . . . 98

5.2.1.3 Existing Tools for Two-Party SFE . . . . . . . . . . . . 99

5.2.2 Tool for Automating Secure Two-partY computations (TASTY) 102

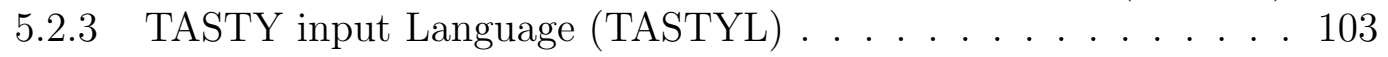

5.2 .3 .1 TASTYL Types and Operators . . . . . . . . . . . . 103

$5.2 .3 .2 \quad$ TASTYL Syntax and Example . . . . . . . . . . . . 105

5.2 .4 Primitives and Optimizations . . . . . . . . . . . . 107

5.2 .5 Performance Measurements . . . . . . . . . . . . . . . . . . . 108

5.2.5.1 Multiplication Protocols . . . . . . . . . . . . . . . 109

5.2.5.2 Evaluation of Fairplay Circuits and AES . . . . . . . . 112

5.3 Application: Privacy-Preserving Face Recognition . . . . . . . . . . . . 113

5.3 .1 Motivation . . . . . . . . . . . . . . . 113

$5.3 .2 \quad$ Face Recognition using Eigenfaces . . . . . . . . . . . . . . . . 115

$5.3 .3 \quad$ Privacy-Preserving Face Recognition . . . . . . . . . . . . . 116

5.3.3.1 Privacy-Preserving Face Recognition using Eigenfaces. 117

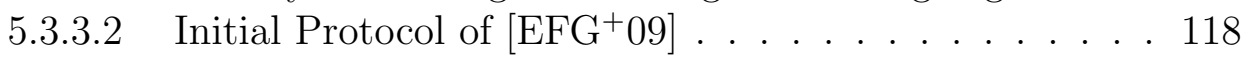

5.3.3.3 Improved Minimum Protocol of [SSW09] . . . . . . . . 120

5.3.3.4 Alternative Minimum Protocol of $\left.\left[\mathrm{BBC}^{+} 10\right]\right]$. . . . . . 120

$5.3 .4 \quad$ A More Improved Hybrid Minimum Protocol . . . . . . . . . . . 122

$5.3 .4 .1 \quad$ Protocol Complexity . . . . . . . . . . . . . . . . 124

5.3.4.2 Performance Comparison . . . . . . . . . . . . . . 124

6 Conclusion 129

6.1 Summary $\ldots \ldots \ldots \ldots$

6.2 Directions for Future Research . . . . . . . . . . . . . . . . . . . . . . . 130

6.2 .1 SFE of Large Functionalities . . . . . . . . . . . . . . . . . . 131

6.2 .2 Automatic Partitioning into Hybrid SFE Protocols . . . . . . . 132

\begin{tabular}{lc}
\hline Bibliography & 133
\end{tabular}

\begin{tabular}{ll}
\hline About the Author & 149
\end{tabular} 


\section{List of Figures}

2.1 Function Representations . . . . . . . . . . . . . . . . . . . . . . . . . . 7

2.2 Interface of GC Constructions . . . . . . . . . . . . . . . . . . . . 16

2.3 Parallel 1-out-of-2 Oblivious Transfer . . . . . . . . . . . . . . . . . . . 20

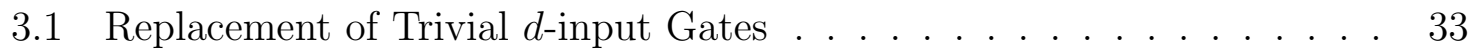

3.2 Replacement of XOR-Trivial 3-input Gates . . . . . . . . . . . . . . . . 38

3.3 Circuit: Addition (ADD) . . . . . . . . . . . . . . . . . . . . . . 40

3.4 Circuit: Subtraction (SUB) . . . . . . . . . . . . . . . . . . . . . . 41

3.5 Circuit: Controlled Addition or Subtraction (ADDSUB) . . . . . . . . . 41

3.6 Size: Multiplication Circuits . . . . . . . . . . . . . . . . . . 43

3.7 Circuit: Comparison (CMP) . . . . . . . . . . . . . . . . . . . . . . . . . . . . . . . 44

3.8 Circuit: Multiplexer (MUX) . . . . . . . . . . . . . . . . . . . . . . . . . . . . . 45

3.9 Circuit: Minimum (MIN) $\ldots \ldots \ldots$. . . . . . . . . . . . . . . . . . . 45

3.10 Block: Minimum $(\min ) \ldots \ldots \ldots \ldots \ldots$

4.1 Model Overview: Token-assisted SFE . . . . . . . . . . . . . . . . . . . 56

4.2 Protocols Overview: Token-Assisted SFE . . . . . . . . . . . . . . . . 58

4.3 Example: Circuit Representation . . . . . . . . . . . . . . . . . . . . 60

4.4 Evaluating a Functionality without Leakage . . . . . . . . . . . . . . 70

4.5 Circuit of $k$ Gates which requires $\Theta(k)$ Memory for Evaluation . . . . . 74

4.6 Architecture: GC Evaluation on Memory-Constrained Devices . . . . . 74

4.7 Example: Circuit and Instruction Sequence. . . . . . . . . . . . . . . . 75

4.8 System Architectures: Hardware-Assisted GC Evaluation . . . . . . . . . 77

4.9 Model: Secure Outsourcing of Data and Arbitrary Computations. . . . . 84

4.10 Architecture: Token Computes [JSM01] . . . . . . . . . . . . . . . . 85

4.11 Architecture: Cloud Computes [GGP10] . . . . . . . . . . . . . . . . . 86

4.12 Our Architecture: Token Sets Up and Cloud Computes . . . . . . . . . 88

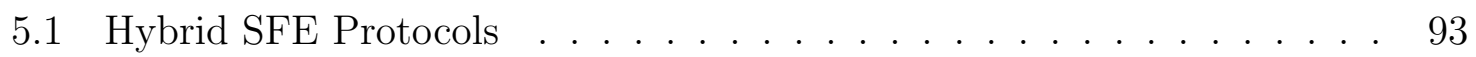

5.2 Architecture and Workflow of TASTY . . . . . . . . . . . . . . . . . 103

5.3 TASTYL: Types and Operators . . . . . . . . . . . . . . . . . . . 104

5.4 TASTYL: Example Program . . . . . . . . . . . . . . . . . . . 106

5.5 Secure Multiplication Protocols: Communication . . . . . . . . . . . . 109

$5.6 \quad$ Secure Multiplication Protocols: Timings . . . . . . . . . . . . . . . . . 111

5.7 Protocol Structure: Secure Face Recognition using Eigenfaces . . . . . 118

5.8 TASTYL: Improved Privacy-Preserving Face Recognition of [SSW09] . 121

5.9 TASTYL: More Improved Privacy-Preserving Face Recognition . . . . . . 123

5.10 Minimum: Communication Complexity (Face-Recognition) . . . . . . . 126

5.11 Minimum: Communication Complexity (Fingerprint Matching) . . . . . 127 


\section{List of Tables}

2.1 Security and Correctness Parameters . . . . . . . . . . . . . . . . 6

2.2 Security Parameters: Recommended Sizes [ECR10] . . . . . . . . . . . 6

2.3 Additively Homomorphic Encryption Schemes . . . . . . . . . . . . . . 11

2.4 Efficient GC Constructions for $d$-input Gates . . . . . . . . . . . . . . . 19

2.5 Complexity of $\mathrm{OT}_{t}^{n}$ in the Random Oracle Model . . . . . . . . . . . . 22

$3.1 \quad$ Number of Trivial $d$-input Gates . . . . . . . . . . . . . . . . . . . . . . 34

3.2 Size: Efficient Circuit Constructions . . . . . . . . . . . . . . . . . . . . 40

3.3 Size: Multiplication Circuits . . . . . . . . . . . . . . . . . . . . 44

3.4 Communication Complexity: Comparison Protocols . . . . . . . . . . . 49

4.1 Secure Protocols using Hardware Tokens . . . . . . . . . . . . . . . . . 55

4.2 Communication Complexity: SW-based vs. Token-assisted SFE . . . . 59

$4.3 \quad$ AES Circuit Optimized for Low Memory . . . . . . . . . . . . . . . . 77

4.4 Timings: Instructions on Prototypes . . . . . . . . . . . . . . . . . . . 78

4.5 Timings: GC Evaluation of AES on Prototypes $\ldots \ldots$. . . . . . . . . . . 79

4.6 Complexity: Architectures for Privacy-Preserving Cloud Computing . . 89

5.1 Abstraction Levels: Automatic Generation of SFE Protocols . . . . . . 99

$5.2 \quad$ Performance Comparison: GC Evaluation of AES . . . . . . . . . . . . 112

$5.3 \quad$ Parameters and Sizes: Privacy-Preserving Face Recognition . . . . . . . 116

\section{List of Algorithms}

$1 \quad$ Optimize Circuit by Removing Constant and 1-input Gates . . . . . . . 35

$2 \quad$ Karatsuba Multiplication . . . . . . . . . . . . . . . . . 42

$3 \quad$ Face Recognition with Eigenfaces [TP91b, TP91a] . . . . . . . . . . . . 117

\section{List of Acronyms}

AE Algorithm Engineering.

AES Advanced Encryption Standard.

DAG Directed Acyclic Graph. 
EC Elliptic Curve.

FPGA Field-Programmable Gate Array.

GC Garbled Circuit.

HE Homomorphic Encryption.

HW hardware.

MAC Message Authentication Code.

OBDD Ordered Binary Decision Diagram.

OT Oblivious Transfer.

OTM One-Time Memory.

OTP One-Time Program.

PRF Pseudo-Random Function.

RAM Random Access Memory.

RO Random Oracle.

SFE Secure Function Evaluation.

SHA Secure Hash Algorithm.

SMPC Secure Multi-Party Computation.

SOPC System On a Programmable Chip.

SW software.

TASTY Tool for Automating Secure Two-partY computations.

TASTYL TASTY input Language.

TPM Trusted Platform Module.

TTP Trusted Third Party.

UC Universal Composability.

UCi Universal Circuit.

VHDL Very high speed integrated circuit Hardware Description Language. 



\section{Chapter 1}

\section{Introduction}

Secure Function Evaluation (SFE), The concept of two-party Secure Function Evaluation (SFE) was introduced in 1982 by Yao [Yao82. The goal of SFE is to let two mutually mistrusting parties compute an arbitrary function $f$ on their private inputs $x, y$ without the help of a Trusted Third Party (TTP) while revealing no information about their inputs beyond the result $f(x, y)$.

Using Garbled Circuits (GCs) [Yao86], an arbitrary function can be computed securely in a constant number of rounds with computation and communication linear in the size of the function. The basic idea is that one party "encrypts" the circuit (using symmetric keys), the other party obliviously obtains the keys corresponding to both parties' inputs and the GC, and is able to decrypt the corresponding output.

An alternative approach is based on Homomorphic Encryption (HE). Here, one party sends its encrypted inputs to the other party, who then computes the desired function under encryption using the homomorphic properties of the cryptosystem. In the end, the encrypted result is sent back and decrypted. Popular homomorphic cryptosystems are the additively homomorphic cryptosystems of Paillier [Pai99] and Damgård-Jurik [DJ01] which require interaction for multiplication. The recently proposed fully homomorphic schemes [Gen09a, Gen09b, SV10, DGHV10] allow both, addition and multiplication under encryption, but still need to be improved to become usable in practical applications.

For many years, these two approaches for SFE, GC and $\mathrm{HE}$, have co-existed with their respective advantages and drawbacks: e.g., GC is highly efficient as mostly based on symmetric key cryptography, but requires linear computation; $\mathrm{HE}$ can result in less communication overhead, but requires expensive public-key operations.

In the last years, SFE was used as enabling technology for a large number of security- and privacy-critical applications (e.g., electronic auctions [NPS99], data mining [LP09b], remote diagnostics [BPSW07, medical diagnostics [BFK ${ }^{+}$09b], or face recognition $\left.\left[\mathrm{EFG}^{+} 09\right]\right)$. To bring such protocols closer to deployment in real-world applications, we apply several ideas from Algorithm Engineering to SFE. 
Algorithm Engineering (AE), 'Algorithm Engineering (AE) is concerned with the design, analysis, implementation, tuning, debugging and experimental evaluation of computer programs for solving algorithmic problems. It provides methodologies and tools for developing and engineering efficient algorithmic codes and aims at integrating and reinforcing traditional theoretical approaches for the design and analysis of algorithms and data structures." DFI03]

The major goals of $\mathrm{AE}$ are to bridge the gap between theory and practice, accelerate the transfer of algorithmic results into applications, and keep the advantages of theoretical treatment such as the generality of the solutions. In particular, AE tries to find algorithms that work well in practice, i.e., are simple, re-usable, and also take constant factors into account. SSan09]

\subsection{Outline}

In this thesis we apply Algorithm Engineering (AE) to Secure Function Evaluation (SFE) in order to engineer efficient SFE protocols for several practical applications. We focus on the following aspects presented in the respective chapters:

Basic Techniques ( $(2)$. We start with a detailed review of state-of-the-art techniques and optimizations for SFE with special focus on their practicability. In particular we are concerned about their performance including constant factors and possibilities for pre-computation. For Garbled Circuits (GCs) we show how most of the complexity can be shifted into a setup phase.

Efficiency $(\S 3)$. As the efficiency of SFE protocols depends on the size of the evaluated function, a small representation of the function should be chosen. We show how to reduce the function size and provide efficient building blocks for standard functionalities.

Deployability $(\S 4)$. When SFE protocols are deployed in real-world systems, available hardware (HW) resources can be used to improve the SFE protocols. Besides using the available $[\mathrm{HW}$ as accelerator for more efficient computation, we also show how secure $\mathrm{HW}$ can be used to make the communication of GC-based SFE protocols independent of the size of the evaluated function.

Usability $(\S 5)$. In order to make cryptographic protocols usable also for non-experts, compilers have been developed that automatically generate protocols from high-level descriptions, e.g., within the CACE project ${ }^{1}\left[\mathrm{BBB}^{+} 09\right]$. Examples for protocols that can be generated automatically include Zero-Knowledge Proofs [Bri04, CRS05, $\mathrm{BBK}^{+} 09$,

\footnotetext{
${ }^{1}$ Computer-Aided Cryptography Engineering http://cace-project.eu
} 
$\mathrm{BBH}^{+}$09, $\mathrm{BCK}^{+} 09, \widehat{\mathrm{BBK}^{+} 09}, \mathrm{ABB}^{+} 10, \mathrm{MEK}^{+} 10$, BKSS10, and Secure Computation [MOR03, MNPS04, NSY04, Sil04, BLW08, KS08b, BLW08, BDNP08, DGKN09, PSS09. While previous compilers for secure computation have been restricted to use either Homomorphic Encryption (HE) or GCs, we present a framework to modularly combine both approaches in a modular and secure way, and provide a novel tool and language for the specification and automatic generation of such hybrid SFE protocols, called Tool for Automating Secure Two-partY computations (TASTY).

\subsection{Summary of Main Results}

The main results of this thesis, presented in the respective chapters, are:

Exactly half of the 2- and 3-input gates can be improved using free XORs.

We show that all 2- and 3-input gates that have an even number of 1-entries in their gate table, can be replaced by a smaller $(d-1)$-input gate and a small number of XOR gates as stated in the following theorem (\$3.2):

Theorem 3 (XOR-trivial gates). For $d \in\{2,3\}$, the XOR-trivial d-input gates are exactly the even gates. Each of them can be replaced by at most one $(d-1)$ input non-XOR gate and at most 3 XOR gates.

As even 3-input gates occur in many common circuit constructions, e.g., addition, subtraction, comparison, or multiplication, we obtain more efficient circuit constructions for these functionalities when XOR gates can be evaluated substantially more efficient than non-XOR gates $(\$ 3.3)$. This results in corresponding performance improvements of protocols that securely evaluate such circuits, e.g., secure comparison and privacy-preserving first-price auctions (\$3.4).

\$4: The amount of data sent between both parties in Garbled Circuit-based Secure Function Evaluation protocols can be made independent of the size of the evaluated function using a resource-limited, tamper-proof hardware token.

We show how Garbled Circuits (GCs) can be generated gate-by-gate using symmetric cryptography and constant memory only. This allows us to implement GC generation within a tamper-proof hardware (HW) token with limited HW resources. Based on this, we develop a token-based Secure Function Evaluation (SFE) protocol where the server issues to the client a tamper-proof $\mathrm{HW}$ token that generates GCs on behalf of the server, but is not trusted by the client (\$4.1). We further show that similar HW tokens can be used to generate One-Time Programs, a method for provably leakage-resilient computation $(\$ 4.2)$, as well as for secure outsourcing of data and arbitrary computations thereon in a cloud computing scenario $(\$ 4.3)$. 
$\S 5$.

Secure Function Evaluation protocols based on (possibly combinations of) both paradigms, Garbled Circuits and Homomorphic Encryption, can be expressed as a sequence of operations on correspondingly encrypted data.

We give a modular framework that combines today's most efficient techniques for SFE of functions in different representations based on Garbled Circuits and Homomorphic Encryption. In our framework, SFE protocols are expressed as a sequence of operations on correspondingly encrypted data: encryption, computation under encryption, possibly conversion into a different type of encryption, and decryption (\$5.1). We develop a specification language for describing such hybrid SFE protocols in our framework and a tool, called Tool for Automating Secure Two-partY computations (TASTY), that automatically generates executable code from such specifications $(\$ 5.2)$. The combination of different SFE techniques allows us to construct efficient protocols for privacy-preserving face recognition based on the Eigenface recognition algorithm (\$5.3). 


\section{Chapter 2}

\section{Secure Function Evaluation Basics}

We start this chapter with common notation and definitions used in this thesis (\$2.1). Afterwards, we summarize efficient cryptographic primitives which are used in stateof-the-art protocols for efficient Secure Function Evaluation (SFE) in $\S 2.2$. Finally, we show how these primitives, in particular Garbled Circuits (GCs), can be used for efficient SFE protocols in the two- and multi-party setting (\$2.3).

Publication Info: A preliminary version of parts of this chapter is included in [KSS10].

\subsection{Common Notation and Definitions}

In this section we introduce common notation (\$2.1.1), cryptographic primitives (\$2.1.2), function representations ( $\$ 2.1 .3$ ), the adversary model (\$2.1.4), and the Random Oracle (RO) model (\$2.1.5) used in this thesis.

\subsubsection{Notation}

We use the following standard notations.

\subsubsection{Basics}

Bitstrings. $\{0,1\}^{\ell}$ denotes the space of binary strings of length $\ell$. $a \| b$ denotes the concatenation of strings $a$ and $b .\langle a, b\rangle$ is a vector with two components $a$ and $b$, and its representation as bit string is $a \| b$.

For strings $s, t \in\{0,1\}^{\ell}, s \oplus t$ denotes their bitwise exclusive-or (XOR).

Random Choice. Uniform random choice is denoted by the $\in_{R}$ operator, e.g., $r \in_{R}$ $D$ reads "draw $r$ uniformly at random from $D$ ". 
Protocol Participants. We call the two Secure Function Evaluation (SFE) participants client $\mathcal{C}$ (Alice) and server $\mathcal{S}(\mathrm{Bob})$. This naming choice is influenced by the asymmetry in the SFE protocols, which fits into the client-server model. We want to point out that we do not limit ourselves to this setting even though this client-server relationship in fact exists in most real-life two-party SFE scenarios.

\subsubsection{Security and Correctness Parameters}

Our security and correctness parameters are named as shown in Table 2.1.

Table 2.1: Security and Correctness Parameters

\begin{tabular}{|c|l|}
\hline Symbol & Name \\
\hline$t$ & symmetric security parameter (bit length of symmetric keys) \\
$T$ & asymmetric security parameter (bit length of RSA moduli) \\
$\sigma$ & statistical security parameter \\
$\kappa$ & correctness parameter \\
\hline
\end{tabular}

Table 2.2 contains current recommendations by ECRYPT II [ECR10] for the size of the symmetric security parameter $t$ and the asymmetric security parameter $T$. An overview and comparison of different recommendations is available at GQ10.

In implementations, the statistical security parameter $\sigma$ and the correctness parameter $\kappa$ can be chosen as $\sigma=\kappa=40$.

Table 2.2: Security Parameters: Recommended Sizes [ECR10]

\begin{tabular}{|l|ccc|}
\hline Security Level & Recommended use until & $t$ & $T$ \\
\hline ultra-short & 2012 & $80 \mathrm{bit}$ & $1,248 \mathrm{bit}$ \\
short & 2020 & $96 \mathrm{bit}$ & $1,776 \mathrm{bit}$ \\
medium & 2030 & $112 \mathrm{bit}$ & $2,432 \mathrm{bit}$ \\
long & 2040 & $128 \mathrm{bit}$ & $3,248 \mathrm{bit}$ \\
\hline
\end{tabular}

\subsubsection{Cryptographic Primitives}

Pseudo-Random Function (PRF) keyed with $k$ and evaluated on $x$ is denoted by $\mathrm{PRF}_{k}(x)$. PRF can be instantiated with a block cipher, e.g., AES, or a cryptographic hash function, e.g., SHA-256. AES is preferable if $\overline{\mathrm{PRF}}$ is run repeatedly with the same key $k$ as in this case the key schedule of AES needs to be run only once and hence amortizes. 
Message Authentication Code (MAC) keyed with $k$ and evaluated on message $m$ is denoted by $\operatorname{MAC}_{k}(m)$. In our token-based protocols in $\$ 4$ we use a MACalgorithm that does not need to store the entire message, but can operate "online" on small blocks, e.g., AES-CMAC [SPLI06] or HMAC KBC97].

\subsubsection{Function Representations}

We use several standard representations for functions which are particularly useful for SFE protocols as shown in Fig. 2.1; boolean circuits (\$2.1.3.1) and arithmetic circuits (\$2.1.3.2.

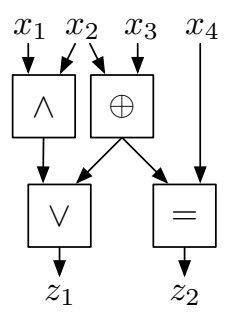

(a) Boolean Circuit

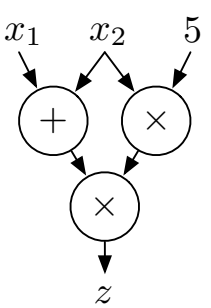

(b) Arithmetic Circuit

Figure 2.1: Function Representations

\subsubsection{Boolean Circuits}

Boolean circuits are a classical representation of functions in engineering and computer science.

Definition 1 (Boolean Circuit). A boolean circuit with $u$ inputs, $v$ outputs and $n$ gates is a Directed Acyclic Graph (DAG) with $|V|=u+v+n$ vertices (nodes) and $|E|$ edges. Each node corresponds to either a gate, an input or an output. The edges are called wires.

For simplicity, the input and output nodes are often omitted in the graphical representation of a boolean circuit as shown in Fig. 2.1(a). For a more detailed definition see Vol99.

Definition 2 (Gate). A d-input gate $G_{d}$ is a boolean function which maps $d \geq 0$ input bits to one output bit, i.e., $G_{d}:\left(\mathrm{in}_{1}, \ldots, \mathrm{in}_{d}\right) \in\{0,1\}^{d} \rightarrow\{0,1\}$.

Typical gates are XOR $(\oplus), \operatorname{XNOR}(=), \operatorname{AND}(\wedge)$, OR $(\vee)$. 
Topologic Order. Gates of a boolean circuit can be evaluated in any order, as long as all of the current gate's inputs are known. This property is ensured by sorting (and evaluating) the gates topologically, which can be done efficiently in $O(|V|+|E|)$ CLRS01, Topological sort, pp. 549-552]. The topologic order of a boolean circuit indexes the gates with labels $G_{1}, \ldots, G_{n}$ and ensures that the $i$-th gate $G_{i}$ has no inputs that are outputs of a successive gate $G_{j>i}$. In complexity theory, a circuit with such a topologic order is called a straight-line program [ALR99]. Given the values of the inputs, the output of the boolean circuit can be evaluated by evaluating the gates one-by-one in topologic order. A valid topologic order for the example boolean circuit in Fig. 2.1(a) would be $\wedge, \oplus, \vee,=$. The topologic order is not necessarily unique, e.g., $\oplus, \wedge,=, \vee$ would be possible as well.

Throughout this thesis we assume that boolean circuits are ordered topologically.

\subsubsection{Arithmetic Circuits}

Arithmetic circuits are a more compact function representation than boolean circuits.

An arithmetic circuit over a ring $R$ and the set of variables $x_{1}, \ldots, x_{n}$ is a DAG. Fig. 2.1(b) illustrates an example. Each node with in-degree zero is called an input gate labeled by either a variable $x_{i}$ or an element in $R$. Every other node is called a gate and labeled by either + or $\times$ denoting addition or multiplication in $R$.

Any boolean circuit can be expressed as an arithmetic circuit over $R=\mathbb{Z}_{2}$. However, if we use $R=\mathbb{Z}_{m}$ for sufficiently large modulus $m$, the arithmetic circuit can be much smaller than its corresponding boolean circuit, as integer addition and multiplication can be expressed as single operations in $\mathbb{Z}_{m}$.

Number Representation. We note that arithmetic circuits can simulate computations on both positive and negative integers $x$ by mapping them into elements of $\mathbb{Z}_{m}$ : $x \mapsto x \bmod m$. As with all fixed precision arithmetics, over- and underflows must be avoided.

\subsubsection{Adversary Model}

The standard approach for formalizing and proving security of cryptographic protocols is to consider adversaries with different capabilities. In the following we give an intuition for the capabilities of malicious, covert, and semi-honest adversaries. We refer to Gol04] for formal definitions and to [LP09b, KSS10] for more detailed discussions.

Malicious adversaries, also called active adversaries, are the strongest type of adversaries and are allowed to arbitrarily deviate from the protocol, aiming to learn private inputs of the other parties and/or to influence the outcome of the computation. Not surprisingly, protection against such attacks is relatively expensive, as discussed in 32.3 .1 .2 . 
Covert adversaries are similar to malicious adversaries, but with the restriction that they must avoid being caught cheating. That is, a protocol in which an active attacker may gain advantage may still be considered secure if attacks are discovered with certain fixed probability (e.g., 1/2). It is reasonable to assume that in many social, political and business scenarios the consequences of being caught overweight the gain from cheating. At the same time, protocols secure against covert adversaries can be substantially more efficient than those secure against malicious players, as shown in $\$ 2.3 .1 .2$

Semi-honest adversaries, also called passive adversaries, do not deviate from the protocol but try to infer additional information from the transcript of messages seen in the protocol. Far from trivial, this model covers many typical practical settings such as protection against insider attacks. Further, designing and evaluating the performance of protocols in the semi-honest model is a first step towards protocols with stronger security guarantees (cf. \$2.3.1.2). Indeed, most protocols and implementations of protocols for practical privacy-preserving applications focus on the semi-honest model [NPS99, $\mathrm{BFK}^{+}$09b, $\mathrm{EFG}^{+}$09, SSW09, OPJM10].

\subsubsection{Random Oracle Model}

Some of our constructions in this thesis make use of Random Oracles (ROs) [BR93, a relatively strong assumption. In fact, it has been shown in [CGH04] that some (contrived) uses of $\mathrm{RO}$ cannot be securely instantiated with any hash function. Therefore, proofs in the $\mathrm{RO}$ model cannot be seen as proofs in the strictest mathematical sense. However, we believe that modeling cryptographic hash functions, such as SHA-256, as $\mathrm{RO}$ is well-justified in many practical settings because of the following reasons:

Firstly, to date, no attacks exploiting the $\mathrm{RO}$ assumption are known on practical systems. This holds true even in the academic context: Important attacks on SHA-1 WYY05] that exploit the structure of the functions were far from being practical, and simply accelerated migration to stronger primitives, which are believed secure today. While some attacks, such as the attack on MD5 [SSA+09], are in fact practical, the use of MD5 had long been considered unsafe, and [SSA $\left.{ }^{+} 09\right]$ broke poorly managed systems. Thus we do not consider [SSA${ }^{+}$09] an attack on properly implemented protocols. In fact, [SSA${ }^{+}$09], and the preliminary works that led to it only support the historic fact that users of hash functions do receive weakness warnings years ahead of possible real breaks.

Further, even in well-understood and deployed real-life systems, the crypto core (which includes the employed hash functions) is almost never targeted for attacks, in favor of much easier to exploit implementation flaws.

In sum, we believe that making the $\mathrm{RO}$ assumption on the employed hash function is practically justified in our and many other settings. 


\subsection{Cryptographic Primitives for Secure Two-Party Computation}

In this section we describe essential building blocks used in Secure Function Evaluation (SFE) protocols: Homomorphic Encryption (HE) in \$2.2.1. Garbled Circuits (GCs) in \$2.2.2, and Oblivious Transfer (OT) in \$2.2.3.

\subsubsection{Homomorphic Encryption (HE)}

Homomorphic Encryption (HE) schemes are semantically secure encryption schemes which allow to compute specific operations on ciphertexts and hence can be used for secure evaluation of arithmetic circuits as described next.

Let (Gen, Enc, Dec) be a semantically secure encryption scheme with plaintext space $P$ ciphertext space $C$, and algorithms for key generation Gen, encryption Enc and decryption Dec. We write $\llbracket m \rrbracket$ for $\operatorname{Enc}(m, r)$, where $r$ is random.

\subsubsection{Additively Homomorphic Encryption}

An additively $\mathrm{HE}$ scheme allows addition under encryption as follows. It defines an operation + on plaintexts and a corresponding operation $\boxplus$ on ciphertexts, satisfying $\forall x, y \in P: \llbracket x \rrbracket \boxplus \llbracket y \rrbracket=\llbracket x+y \rrbracket$. This naturally allows for multiplication with a plaintext constant $a$ using repeated doubling and adding: $\forall a \in \mathbb{N}, x \in P: a \llbracket x \rrbracket=\llbracket a x \rrbracket$.

Popular instantiations for additively HE schemes are summarized in Table 2.3. The Paillier cryptosystem [Pai99] provides a $T$-bit plaintext space and $2 T$-bit ciphertexts, where $T$ is the size of the RSA modulus $N$, and is sufficient for most applications (details see below). The Damgård-Jurik cryptosystem [DJ01] is a generalization of the Paillier cryptosystem which provides a larger plaintext space of size $s T$-bit for $(s+1) T$ bit ciphertexts for arbitrary $s \geq 1$. The Damgård-Geisler-Krøigaard cryptosystem [DGK07, DGK08b, DGK08a] has smaller ciphertexts of size $T$-bit, but can be used with a small plaintext space $\mathbb{Z}_{u}$ only, where $u$ is a small prime, as decryption requires to compute a discrete logarithm. Finally, the lifted ElGamal Gam85] cryptosystem has additively homomorphic properties and very small ciphertexts. However, decryption is only possible if the plaintext is known to be in a small subset of the plaintext space, as the discrete logarithm of a generator with large order has to be brute-forced. Lifted ElGamal implemented over an Elliptic Curve (EC) group (Lifted EC-ElGamal) provides a $2 t$-bit plaintext space and very small ciphertexts of size $2(2 t+1)$ bits $\sim 4 t$ bits when using point compression.

The Paillier Cryptosystem. The most widely used additively $\mathrm{HE}$ system is that of Paillier [Pai99] where the public key is an RSA modulus $N$ and the secret key is the factorization of $N$. The extension of [DJ01, Sect. 6] allows to pre-compute expensive modular exponentiations of the form $r^{N} \bmod N^{2}$ in a setup phase, s.t. only two 
modular multiplications per encryption are needed in the time-critical online phase. The party which knows the factorization of $N$ (i.e., the secret key), can use Chinese remaindering to efficiently pre-compute these exponentiations and to decrypt.

Table 2.3: Additively Homomorphic Encryption Schemes ( $N$ : RSA modulus; $s \geq 1 ; u$ : small prime; $p$ : $2 t$-bit prime; $g, h$ : generators)

\begin{tabular}{|l||c|c|c|}
\hline Scheme & $P$ & Ciphertext size & Enc $(m, r)$ \\
\hline \hline Paillier [Pai99] & $\mathbb{Z}_{N}$ & $2 T$ & $g^{m} r^{N} \bmod N^{2}$ \\
Damgård-Jurik [DJ01] & $\mathbb{Z}_{N^{s}}$ & $(s+1) T$ & $g^{m} r^{N^{s}} \bmod N^{s+1}$ \\
Damgård-Geisler-Krøigaard [DGK07] & $\mathbb{Z}_{u}$ & $T$ & $g^{m} h^{r} \bmod N$ \\
Lifted EC-ElGamal [Gam85] & $\mathbb{Z}_{p}$ & $4 t+2$ & $\left(g^{r}, g^{m} h^{r}\right)$ \\
\hline
\end{tabular}

\subsubsection{Fully Homomorphic Encryption}

Some $\mathrm{HE}$ systems allow both addition and multiplication under encryption. For this, a separate operation $\times$ for multiplication of plaintexts and a corresponding operation $\bigotimes$ on ciphertexts is defined satisfying $\forall x, y \in P: \llbracket x \rrbracket \bigotimes \llbracket y \rrbracket=\llbracket x \times y \rrbracket$. Cryptosystems with such a property are called fully homomorphic.

Until recently, it was widely believed that such cryptosystems do not exist. Several works provided partial solutions: [BGN05, GHV10] allow for polynomially many additions and one multiplication, and ciphertexts of [SYY99] grow exponentially in the number of multiplications. Recent schemes [Gen09a, Gen09b, SV10, DGHV10] are fully homomorphic. Even if the size of the ciphertexts of these fully HE schemes is independent of the number of multiplications, the size and computational cost of fully $\mathrm{HE}$ schemes are substantially larger than those of additively $\mathrm{HE}$ schemes. First implementation results of [SV10] show that even for almost fully HE schemes with conservatively chosen security parameters that allow for multiplicative depth $d=2.5$ of the evaluated circuit, i.e., at most two multiplications, encrypting a single bit takes $3.7 \mathrm{~s}$ on a $2.4 \mathrm{GHz}$ Intel Core2 (6600) CPU. Recently, Craig Gentry and Shai Halevi presented the first working implementation of fully $\mathrm{HE}$ - i.e., an implementation of the entire bootstrapping procedure - together with performance numbers in the order of Gigabytes of communication and minutes of computation on high-end IBM System x3500 servers [GH10.

Although significant effort is underway in the theoretical community to improve its performance, it seems unlikely that fully $\mathrm{HE}$ would reach the efficiency of current public-key encryption schemes. Intuitively, this is because a fully HE cryptosystem must provide both the same strong security guarantees (semantic security) and extra algebraic structure to allow for homomorphic operations. The extra structure weakens security, and countermeasures (costing performance) are necessary. 


\subsubsection{Computing on Encrypted Data}

Homomorphic encryption naturally allows for secure evaluation of arithmetic circuits over $P$, called computing on encrypted data, as follows. The client $\mathcal{C}$ generates a key pair for a $\mathrm{HE}$ cryptosystem and sends her inputs encrypted under the public key to the server $\mathcal{S}$ together with the public key. With a fully $\mathrm{HE}$ scheme, $\mathcal{S}$ can simply evaluate the arithmetic circuit by computing on the encrypted data and send back the (encrypted) result to $\mathcal{C}$, who then decrypts it to obtain the output? 1 If the HE scheme only supports addition, one round of interaction between $\mathcal{C}$ and $\mathcal{S}$ is needed to evaluate each multiplication gate (or a layer of multiplication gates) as described below. Today, using additively $\mathrm{HE}$ and interaction for multiplication results in much faster SFE protocols than using fully $\mathrm{HE}$ schemes.

Packing. Often the plaintext space $P$ of the HE scheme is substantially larger than the size of encrypted numbers. This allows to pack multiple numbers into one ciphertext (before or after additive blinding) and send back only a single ciphertext from $\mathcal{S}$ to $\mathcal{C}$. This optimization substantially decreases the size of the messages sent from $\mathcal{S}$ to $\mathcal{C}$ as well as the number of decryptions performed by $\mathcal{C}$. The computational overhead for $\mathcal{S}$ is small as packing the ciphertexts $\llbracket x_{1} \rrbracket, \ldots, \llbracket x_{n} \rrbracket$ into one ciphertext $\llbracket X \rrbracket=\llbracket x_{n}\|\ldots\| x_{1} \rrbracket$ costs less than one full-range modular exponentiation by using Horner's scheme: $\llbracket X \rrbracket=\llbracket x_{n} \rrbracket$; for $i=n-1$ downto $1: \llbracket X \rrbracket=2^{\left|x_{i+1}\right|} \llbracket X \rrbracket \boxplus \llbracket x_{i} \rrbracket$.

Interactive Multiplication with Additively Homomorphic Encryption. To multiply two $\ell$-bit values encrypted under additively $\mathrm{HE}$ and held by $\mathcal{S}, \llbracket x \rrbracket$ and $\llbracket y \rrbracket$, the following standard protocol requires one single round of interaction between $\mathcal{S}$ and $\mathcal{C}: \mathcal{S}$ randomly chooses $r_{x}, r_{y} \in_{R}\{0,1\}^{\ell+\sigma}$, where $\sigma$ is the statistical security

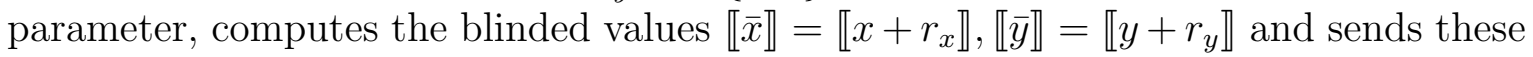
to $\mathcal{C}$. $\mathcal{C}$ decrypts, multiplies and sends back $\llbracket z \rrbracket=\llbracket \bar{x} \bar{y} \rrbracket$. $\mathcal{S}$ obtains $\llbracket x y \rrbracket$ by computing $\llbracket x y \rrbracket=\llbracket z \rrbracket \boxplus\left(-r_{x}\right) \llbracket y \rrbracket \boxplus\left(-r_{y}\right) \llbracket x \rrbracket \boxplus \llbracket-r_{x} r_{y} \rrbracket$.

Efficiency of single or multiple multiplications in parallel can be improved by packing the blinded ciphertexts together instead of sending them to $\mathcal{C}$ separately.

Security. We note that in $\mathrm{SFE}$ protocols based on $\mathrm{HE}$, the security of the party who knows the secret key ( $\mathcal{C}$ in our setting) is computational as it is computationally hard for the other party to break the semantic security of the $\mathrm{HE}$ scheme. The security of the party who computes under $\mathrm{HE}$ ( $\mathcal{S}$ in our setting) is statistical as this party always statistically blinds all intermediate values before sending them back.

${ }^{1}$ If $\mathcal{S}$ is malicious, it must additionally be ensured that he indeed computed the intended functionality by means of verifiable computing (cf. 4 4.3.3.2). 
Efficiency. As SFE based on additively $\mathrm{HE}$ requires interaction for multiplying two ciphertexts, the round complexity of such protocols is determined by the multiplicative depth of the evaluated function, i.e., the number of successive multiplications.

When the public key is known to both parties, encryptions and re-randomization values can be pre-computed already in a setup phase.

Still, the online phase requires computationally expensive public key operations such as modular exponentiations for multiplying with constants, or decryptions.

\subsubsection{Garbled Circuit (GC) Constructions}

Another efficient method for computing under encryption is based on Garbled Circuits (GCs). The fundamental idea of GCs going back to Yao [Yao86, is to represent the function $f$ to be evaluated as boolean circuit $C$ and encrypt (garble) each wire with a symmetric encryption scheme. In contrast to Homomorphic Encryption (HE) (cf. \$2.2.1), the encryptions here cannot be operated on directly, but require helper information which is generated and exchanged in a setup phase in form of a garbled table for each gate.

In this section we summarize existing schemes for constructing and evaluating GCs and give applications in $\$ 2.3 .1$. We give an algorithmic description of GCs and refer to the original papers on GC constructions and [LP09a] for details and proofs of security.

\subsubsection{Components of GC Constructions}

We start with briefly introducing the main components of GC constructions: garbled values and garbled tables to compute thereon.

Garbled Values. Computations in a $\mathrm{GC}$ are not performed on plain values 0 or 1 , but on random-looking secrets, called garbled values. During construction of the $\mathrm{GC}$, two random-looking garbled values $\widetilde{w}_{i}^{0}, \widetilde{w}_{i}^{1}$ are assigned to each wire $w_{i}$ of $C$. The garbled value $\widetilde{w}_{i}^{j}$ corresponds to the plain value $j$, but, as it looks random, does not reveal its corresponding plain value $j$.

In efficient GC constructions, each garbled value is composed of a symmetric $t$-bit key and a random-looking permutation bit (see Point-and-Permute below):

$$
\widetilde{w}_{i}^{0}=\left\langle k_{i}^{0}, \pi_{i}^{0}\right\rangle, \widetilde{w}_{i}^{1}=\left\langle k_{i}^{1}, \pi_{i}^{1}\right\rangle \text { with } k_{i}^{0}, k_{i}^{1} \in\{0,1\}^{t}, \pi_{i}^{0} \in\{0,1\}
$$

and

$$
\pi_{i}^{1}=1-\pi_{i}^{0}
$$

The exact method for choosing the values $k_{i}^{0}, k_{i}^{1}, \pi_{i}^{0}$ is determined by the specific GC construction (cf. \$2.2.2.3). 
Garbled Tables. To allow computations on garbled values, for each gate $G_{i}$ ( $i=$ $1, \ldots, n)$ of the circuit $C$, a garbled table $\widetilde{G}_{i}$ is constructed. Given the garbled values corresponding to $G_{i}$ 's input wires, $\widetilde{G}_{i}$ allows to decrypt only the corresponding garbled value of $G_{i}$ 's output wire. Formally, let $\mathrm{in}_{1}, \ldots, \mathrm{in}_{d}$ be the input wires of gate $G$ and out be its output wire. Then, for any input combination $b_{j} \in\{0,1\}(j=1, \ldots, d)$, given the corresponding garbled inputs $\tilde{\mathrm{in}}_{1}^{b_{1}}, \ldots, \widetilde{\mathrm{in}}_{d}^{b_{d}}$, the garbled table $\widetilde{G}$ allows to decrypt only $\widetilde{\text { out }}\left(b_{1}, \ldots, b_{d}\right)$. In particular, no information about the other garbled output value, the plain input bits $b_{j}$, or the plain output bit $G\left(b_{1}, \ldots, b_{d}\right)$ is revealed.

The general idea for constructing garbled tables is to use for all possible input combinations $b_{j}$ the garbled input keys $\widetilde{\sim}_{j}^{b_{j}}$ to symmetrically encrypt the corresponding output key $\widetilde{\text { out }}\left(b_{1}, \ldots, b_{d}\right)$. The entries of the garbled table are the ciphertexts for all possible input combinations. The position of the entries in the garbled table must be such that it does not reveal any information about the corresponding plain input values $b_{j}$.

To achieve this, the original GC construction proposed by Yao [Yao86] randomly permutes the entries in the garbled table. In order to find the right entry to decrypt, the symmetric encryption function requires an efficiently verifiable range to determine which entry was decrypted successfully as described in LP09a. However, this method has a large overhead as multiple trial-decryption need to be performed and ciphertext size increases.

In the following we briefly discuss the state of the art for efficiently instantiating the used symmetric encryption function.

Point-and-Permute. The point-and-permute technique, as described in [NPS99], allows to immediately find the right entry for decryption in the garbled table as follows: The entries of the garbled table are permuted such that the permutation bits of the garbled input wires $\pi_{1}, \ldots, \pi_{d}$ are used to point directly to the entry of the garbled table which needs to be decrypted. As the permutation bits look random, the position of the entries in the garbled table appears random as well and hence reveals no information about the input bits $b_{1}, \ldots, b_{d}$.

By applying the point-and-permute technique, the employed symmetric encryption scheme no longer needs to have an efficiently verifiable range.

Encryption Function. The encryption function for encrypting garbled table entries is $E_{k_{1}, \ldots, k_{d}}^{s}(m)$ with inputs $d$ keys of length $t$, a message $m$, and some additional information $s$. The additional information $s$ must be unique per invocation of the encryption function, i.e., it is used only once for any choice of keys. Indeed, it is crucial that in the GC constructions $s$ contains a unique and independent gate id (cf. [TX03b]).

As proposed in [LPS08, PSSW09], $E$ can be instantiated efficiently with a Key 
Derivation Function $\mathrm{KDF}^{\ell}\left(k_{1}, \ldots, k_{d}, s\right)$ whose $\ell$ bits of output are independent of the input keys $k_{1}, \ldots, k_{d}$ in isolation, and which depends on the value of $s$ :

$$
E_{k_{1}, \ldots, k_{d}}^{s}(m)=m \oplus \mathrm{KDF}^{|m|}\left(k_{1}, \ldots, k_{d}, s\right) .
$$

KDF can be instantiated using a suitably chosen cryptographic hash function $H$, e.g., from the SHAffamily:

- When $H$ is modeled as Random Oracle (RO), KDF can be instantiated as

$$
\operatorname{KDF}^{\ell}\left(k_{1}, \ldots, k_{d}, s\right)=H\left(k_{1}\|\ldots\| k_{d} \| s\right)_{1 \ldots \ell} .
$$

- Otherwise, KDF requires $d$ separate calls to $H$ as

$$
\operatorname{KDF}^{\ell}\left(k_{1}, \ldots, k_{d}, s\right)=H\left(k_{1}|| s\right)_{1 \ldots \ell} \oplus \cdots \oplus H\left(k_{d} \| s\right)_{1 \ldots \ell} .
$$

In this case, depending on whether the GC construction supports "free XOR" gates or not, KDF needs to be modeled to be correlation robust or a pseudorandom function as described in $\$ 2.2 .2 .3$.

\subsubsection{Interfaces and Structure of GC Constructions}

GC constructions can be seen as algorithms with clean interfaces and a common general structure as described next.

Interface of GC Constructions. Each GC construction consists of two randomized algorithms: createGC generates a $\mathrm{GC}$ and evalGC evaluates it as shown in Fig. 2.2.

- createGC takes a boolean circuit $C$ as input and outputs the corresponding GC $\widetilde{C}$ (consisting of a garbled table for each of its gates), and pairs of garbled values for each of $C$ 's input and output wires.

- evalGC gets as inputs $C, \widetilde{C}$ and one garbled value for each of $C$ 's inputs, $\tilde{\mathrm{in}}_{1}, \ldots, \tilde{\mathrm{in}}_{u}$ and returns the corresponding garbled output values $\widetilde{\text { out }}_{1}, \ldots$, out $_{v}$.

We note that the inputs and outputs of both algorithms can be streams of data, i.e., given piece-by-piece without ever storing the entire objects.

Completeness and Correctness. Each GC construction must be complete and correct. Completeness requires that for all boolean circuits $C$, createGC creates a $\mathrm{GC} \widetilde{C}$, pairs of garbled inputs and garbled outputs. Correctness requires that afterwards for all possible input bits $x_{i} \in\{0,1\}, i=1, \ldots, u$, given the corresponding garbled values $\widetilde{\mathrm{in}}_{i}=\widetilde{\mathrm{in}}_{i}^{x_{i}}$ as inputs, evalGC outputs the garbled values $\widetilde{\mathrm{out}_{j}}=\widetilde{\mathrm{out}}_{j}^{z_{j}}, j=1, \ldots, v$ which correspond to $C$ evaluated on the input values: $\left(z_{1}, \ldots, z_{v}\right)=C\left(x_{1}, \ldots, x_{u}\right)$.

One-time use. We stress that for security reasons, $\widetilde{C}$ cannot be evaluated more than once (otherwise, multiple runs of evalGC can leak information about input or output values). evalGC must always be run on freshly generated outputs of createGC. 


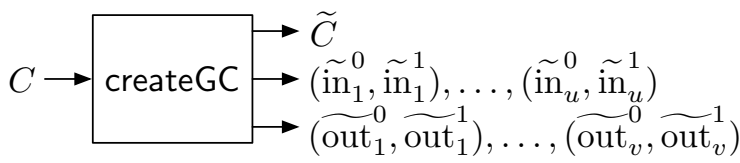

(a) create

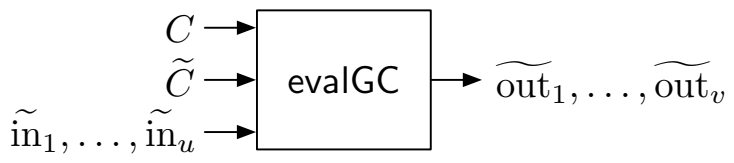

(b) evaluate

Figure 2.2: Interface of GC Constructions

General Structure of GC Constructions. The efficient GC constructions presented next have the following general structure:

- createGC starts with assigning random-looking garbled values $\left(\widetilde{\mathrm{in}}_{i}^{0}, \widetilde{\mathrm{in}}_{i}^{1}\right)$ to all input wires of $C$ and outputs these. Afterwards, for each gate $G_{i}$ of $C$ in topologic order (cf. assumption in 2.1.3.1), two random-looking garbled values are assigned to the gate's output wire and afterwards its garbled table $\widetilde{G}_{i}$ is created and output as part of $\widetilde{C}$. Finally, the garbled outputs $\left(\widetilde{\text { out }_{j}}{ }^{\text {out }}{ }_{j}\right)$ for each of $C$ 's output wires are output.

- evalGC evaluates the $\mathrm{GC} \widetilde{C}$ on the garbled inputs $\tilde{\mathrm{in}}_{i}$ by evaluating each garbled gate $\widetilde{G}_{i}$ of $\widetilde{C}$ in the topologic order determined by $C$. Finally, the garbled values of $C$ 's outputs wires out ${ }_{j}$ are output.

\subsubsection{Efficient GC Constructions}

In the following we describe efficient GC constructions which are suited well for efficient implementation [PSSW09].

All GC constructions presented next start with choosing the garbled input values. The garbled zero values ${\widetilde{\mathrm{in}_{i}}}_{i}^{0}$ are chosen randomly, i.e., $k_{i}^{0} \in_{R}\{0,1\}^{t}$ and $\pi_{i}^{0} \in_{R}\{0,1\}$ in Equation 2.1). The corresponding garbled one values $\widetilde{\mathrm{in}}_{i}^{1}$ are chosen randomly as $k_{i}^{1} \in_{R}\{0,1\}^{t}$, or according to Equation (2.8) in case of "free XOR" as described below.

The following GC techniques successively fix the garbled output values of each gate in order to decrease the size of the garbled tables.

Point-and-Permute. The point-and-permute GC construction was first described in [NPS99], implemented in Fairplay [MNPS04], and also used in [KS08a, LPS08] $]^{2}$.

\footnotetext{
${ }^{2}$ We note that the $\mathrm{GC}$ construction of [YLP05, Sect. 3.3] is less efficient as garbled tables are larger and require slightly more computation.
} 
This technique chooses both garbled output values of a $d$-input gate $G_{i}$ at random and results in a garbled table with $2^{d}$ table entries. For each of the $2^{d}$ possible input combinations $b_{1}, \ldots, b_{d}$, the garbled table entry at position $\pi_{1}, \ldots, \pi_{d}$ is constructed by using the keys of $G_{i}$ 's garbled inputs to encrypt the corresponding garbled output:

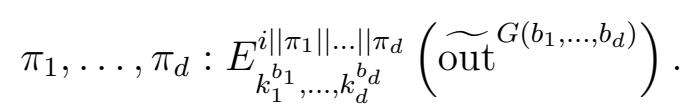

Garbled Row Reduction. The GC construction of [NP01, called Garbled Row Reduction, extends the point-and-permute GC construction by fixing one of the garbled output values resulting in a garbled table of $2^{d}-1$ table entries. The first entry of each garbled table is forced to be zero and hence does not need to be transferred. By substituting into Equation (2.6), this fixes one of the two garbled output values to be pseudo-randomly derived from the garbled input values. The other garbled output value is chosen at random satisfying Equation (2.2). For details we refer to the description in [PSSW09, Sect. 4].

Secret-Sharing. The GC construction of [PSSW09] uses Shamir's secret-sharing Sha79] to fix both garbled output values resulting in a garbled table with $2^{d}-2$ entries. In the following we summarize the general idea of this construction and refer to [PSSW09, Sect. 5] for details.

The construction exploits that both keys of a gate's garbled output values can be chosen independently and pseudo-randomly. The basic idea is to pseudo-randomly derive keys $K_{r} \in\{0,1\}^{t}$ and bit masks $M_{r} \in\{0,1\}$ for all combinations of garbled inputs as

$$
K_{r} \| M_{r}=\mathrm{KDF}^{t+1}\left(k_{1}^{b_{1}}\|\ldots\| k_{d}^{b_{d}} \| s\right) .
$$

The keys $K_{r}$ are interpreted as elements in $\mathbb{F}_{2^{t}}$ and used as supporting points of two polynomials $P(X), Q(X)$ of the same degree: $P(X)$ is defined by those keys which should map to the garbled output value out $\widetilde{o u}^{1}:=P(0)$. Similarly, $Q(X)$ maps to the garbled output value out $\widetilde{o t}^{0}:=Q(0)$. Overall, $2^{d}-2$ points are stored as part of the garbled table, where some points are on both and some on only one of the polynomials. The bits $M_{r}$ are used to encrypt the permutation bits of the garbled outputs as in the point-and-permute GC construction resulting in additional $2^{d}$ encrypted bits in the garbled table.

During evaluation of the garbled gate, the garbled inputs are used to derive $K_{r}, M_{r}$ according to Equation 2.7). Then, $M_{r}$ is used to decrypt the output permutation bit which defines through which of the supporting points in the garbled table to interpolate the polynomial. Finally, the garbled output key is determined by evaluating the polynomial at $X=0$. 
Generalization to arbitrary d. We note that the Secret-Sharing GC construction can be generalized from $d=2$ (as described in [PSSW09, Sect. 5]) to arbitrary $d$ input gates as follows: Assume that $n_{1}$ of the $2^{d}$ entries in the gate's function table equal one and the remaining $n_{0}:=2^{d}-n_{1}$ entries equal zero. In the following we assume that $n_{1} \geq n_{0}$ (otherwise we invert the role of zero and one). The polynomial $P$, interpolated through those keys $K_{r}$ that should map to the garbled output value for one, has degree $n_{1}$. We store $n_{1}-1$ extra points $P\left(2^{d}+1\right), \ldots, P\left(2^{d}+n_{1}-1\right)$ in the garbled table. Afterwards, we interpolate polynomial $Q$ of degree $n_{1}$ through the $n_{0}$ keys $K_{r}$ that should map to the zero garbled output value and the common $n_{1}-n_{0}$ extra points $P\left(2^{d}+1\right), \ldots, P\left(2^{d}+n_{1}-n_{0}\right)$. Now, we create $n_{0}-1$ extra points $Q\left(2^{d}+n_{1}-n_{0}+1\right), \ldots, Q\left(2^{d}+n_{1}-1\right)$. The order of the extra points on $P$ and $Q$ in the garbled table is such that the output permutation bit can be used to obliviously index which extra points to use for interpolation. The garbled table consists of $n_{1}-n_{0}$ common extra points and $n_{0}-1$ extra points on $P$ resp. $Q$, in total $n_{1}-n_{0}+2\left(n_{0}-1\right)=n_{1}+n_{0}-2=2^{d}-2$ keys. The overall size of the garbled table hence is $\left(2^{d}-2\right) t+2^{d}$ bits.

Free XOR. As observed in [KS08a], a fixed distance between corresponding garbled values allows "free" evaluation of XOR gates, i.e., garbled XOR gates require no garbled table and allow very efficient creation and evaluation (XOR of the garbled values). The main idea is to choose a fixed relation between the two garbled values for each garbled wire:

$$
k_{i}^{1}:=k_{i}^{0} \oplus \Delta
$$

where $\Delta \in_{R}\{0,1\}^{t}$ is the randomly chosen global key distance. During creation of

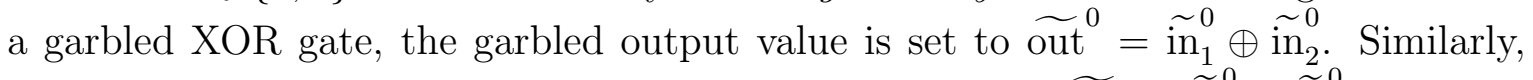
evaluation of a garbled XOR gates is done by computing out $=\widetilde{\mathrm{in}_{1}} \oplus \underset{\tilde{\mathrm{in}}_{2}}{0}$. Garbled non-XOR gates can be constructed with any GC construction which fixes at most one of the garbled outputs of a gate, i.e., from the GC techniques described above Pointand-Permute and Garbled Row Reduction allow combination with "free XORs", but not the Secret-Sharing technique (cf. Table 2.4).

Security. For "free XOR" the keys of all pairs of garbled values are related according to Equation (2.8). Therefore, it is no longer sufficient to model KDF as pseudo-random function. Instead, one has to resort to the stronger notion of correlation robustness of [IKNP03]. For a detailed discussion on how to model KDF we refer to [PSSW09]. A less efficient instantiation of the encryption function which is provably secure in the standard model is given in AHI11. 


\subsubsection{Complexity of Efficient GC Constructions}

The complexity of the GC constructions presented in $\$ 2.2 .2 .3$ is summarized in Table 2.4. When using free XORs, XOR gates require no communication and only negligible computation (XOR of bitstrings). We compare the complexity for other gates next.

Table 2.4: Efficient GC Constructions for $d$-input Gates ( $t$ : symmetric security parameter)

\begin{tabular}{|l||c|c|}
\hline GC Technique & $\begin{array}{c}\text { Size of garbled table } \\
{[\mathrm{bits}]}\end{array}$ & $\begin{array}{c}\text { Free XOR } \\
{[\text { KS08a] }}\end{array}$ \\
\hline Point-and-Permute [NPS99] & $2^{d} t+2^{d}$ & $\checkmark$ \\
Garbled Row Reduction [NPS99] & $\left(2^{d}-1\right) t+\left(2^{d}-1\right)$ & $\sqrt{ }$ \\
Secret-Sharing [PSSW09] & $\left(2^{d}-2\right) t+2^{d}$ & $\boldsymbol{X}$ \\
\hline
\end{tabular}

Computation Complexity. Interestingly, all GC constructions have almost the same computation complexity, which is dominated by invocations of a cryptographic hash function $H$ : for each $d$-input gate, createGC requires $2^{d}$ invocations of KDF and evalGC requires one invocation. As described in $\$ 2.2 .2 .1$, each invocation of KDF needs one or $d$ invocations of $H$ depending on whether $H$ is modeled as $\mathrm{RO}$ or not.

The Secret-Sharing GC construction requires slightly more computations as it also requires to interpolate two polynomials of degree at most $2^{d}-1$ over $\mathbb{F}_{2^{t}}$.

On the other hand, the computation complexity to randomly choose the garbled output values of the gates decreases as follows: Point-and-Permute chooses both garbled values (one with free XOR), Garbled Row Reduction one (none with free XOR), and Secret-Sharing none.

Communication Complexity. As shown in Table 2.4 the size of each garbled table decreases by approximately $t$ bits per gate from Point-and-Permute to Garbled Row Reduction and from there to Secret-Sharing. Especially for gates with low degree $d$ these savings can be quite significant, i.e., up to $-25 \%$ for Garbled Row Reduction and $-50 \%$ for Secret-Sharing for the common case of $d=2$.

However, the Secret-Sharing construction, which cannot be combined with Free XOR, results only in better communication complexity than Garbled Row Reduction if evaluated circuits do not have many XOR gates. Indeed, we show in $\$ 3$ that most commonly used circuit building blocks can be transformed such that most of the gates are XOR gates and hence Garbled Row Reduction is more efficient than Secret-Sharing w.r.t. both, computation and communication. 


\subsubsection{Oblivious Transfer (OT)}

Parallel 1-out-of-2 OT of $n t^{\prime}$-bit strings, denoted as $\mathrm{OT}_{t^{\prime}}^{n}$, is a two-party protocol run between a chooser (client $\mathcal{C}$ ) and a sender (server $\mathcal{S}$ ) as shown in Fig. 2.3. For $i=1, \ldots, n, \mathcal{S}$ inputs $n$ pairs of $t^{\prime}$-bit strings $s_{i}^{0}, s_{i}^{1} \in\{0,1\}^{t^{\prime}}$ and $\mathcal{C}$ inputs $n$ choice bits $b_{i} \in\{0,1\}$. At the end of the protocol, $\mathcal{C}$ learns the chosen strings $s_{i}^{b_{i}}$, but nothing about the other strings $s_{i}^{1-b_{i}}$, while $\mathcal{S}$ learns nothing about $\mathcal{C}$ 's choices $b_{i}$.

In the following, we assume that $\mathrm{OT}$ is used in the context of SFE protocols (as described later in $\$ 2.3 .1$, i.e., the transferred strings are garbled values with length $t^{\prime}=t+1 \sim t$ where $t$ is the symmetric security parameter.

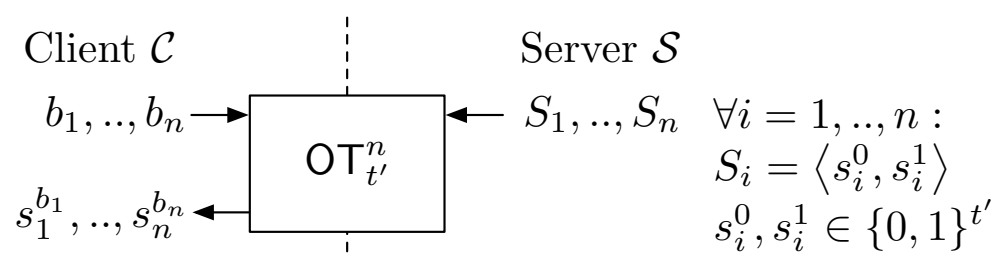

Figure 2.3: Parallel 1-out-of-2 Oblivious Transfer of $n t^{\prime}$-bit Strings $\left(\mathrm{OT}_{t^{\prime}}^{n}\right)$

We describe techniques to efficiently implement OT next.

\subsubsection{Efficient $\mathrm{OT}$ Protocols}

$\mathrm{OT}_{t^{\prime}}^{n}$ can be instantiated efficiently with different protocols, e.g., [NP01, AIR01].

For example the protocol of [NP01 implemented over a suitably chosen EC consists of three messages $(\mathcal{S} \rightarrow \mathcal{C} \rightarrow \mathcal{S} \rightarrow \mathcal{C})$ in which $2 n+1$ EC points and $2 n t^{\prime}$ encrypted bits are sent. Using point compression, each point can be represented with $2 t+1$ bits and hence the overall communication complexity of this protocol is $(2 n+1) \cdot(2 t+1)+$ $2 n t^{\prime}$ bits $\approx 6 n t$ bits. As computation, $\mathcal{S}$ performs $2 n+1$ point multiplications and $2 n$ invocations of a cryptographic hash function $H$, modeled as $\mathrm{RO}$, and $\mathcal{C}$ performs $2 n$ point multiplications and $n$ invocations of $H$. This protocol is provably secure against malicious $\mathcal{C}$ and semi-honest $\mathcal{S}$ in the $\mathrm{RO}$ model.

Similarly, the protocol of [AIR01] implemented over a suitably chosen EC using point compression has communication complexity $n(6(2 t+1))+(2 t+1)$ bits $\sim 12 n t$ bits and is secure against malicious $\mathcal{C}$ and semi-honest $\mathcal{S}$ in the standard model as described in [KSS09].

\subsubsection{Extending OT Efficiently}

The extensions of [IKNP03] can be used to reduce the number of computationally expensive public-key operations of $\mathrm{OT}_{t^{\prime}}^{n}$ to be independent of $n:^{3}$ The transformation

\footnotetext{
${ }^{3}$ This is the reason for our choice of notation $\mathrm{OT}_{t^{\prime}}^{n}$ instead of $n \times \mathrm{OT}^{t^{\prime}}$.
} 
for semi-honest $\mathcal{C}$ reduces $\mathrm{OT}_{t^{\prime}}^{n}$ to $\mathrm{OT}_{t}^{t}$ (with roles of $\mathcal{C}$ and $\mathcal{S}$ swapped) and a small additional overhead: one additional message, $2 n\left(t^{\prime}+t\right)$ bits additional communication, and $\mathcal{O}(n)$ invocations of a correlation robust hash function $H(2 n$ for $\mathcal{S}$ and $n$ for $\mathcal{C}$ ) which is substantially cheaper than $\mathcal{O}(n)$ public-key operations. A slightly less efficient $\mathrm{OT}$ extension for malicious $\mathcal{C}$ is given in [KNP03] and improved in [Nie07].

\subsubsection{Pre-Computing $\mathrm{OT}$}

All computationally expensive operations for OT can be shifted into a setup phase by pre-computing $\mathrm{OT}$ as described in [Bea95]: In the setup phase, the parallel OT protocol is run on randomly chosen values $r_{i} \in_{R}\{0,1\}$ by $\mathcal{C}$ and $m_{i}^{j} \in\{0,1\}^{t^{\prime}}$ by $\mathcal{S}$. In the online phase, $\mathcal{C}$ uses her random bits $r_{i}$ to mask her private inputs $b_{i}$, and sends the masked bits to $\mathcal{S}$. $\mathcal{S}$ replies with encryptions of his private inputs $s_{i}^{j}$ using his random masks $m_{i}^{j}$ from the setup phase. Which input of $\mathcal{S}$ is masked with which random value is determined by $\mathcal{C}$ 's message. Finally, $\mathcal{C}$ applies the masks $m_{i}$ she received from the OT protocol in the setup phase to decrypt the correct output values $s_{i}^{b_{i}}$.

More precisely, the setup phase works as follows: For $i=1, \ldots, n, \mathcal{C}$ chooses random bits $r_{i} \in_{R}\{0,1\}$ and $\mathcal{S}$ chooses random masks $m_{i}^{0}, m_{i}^{1} \in_{R}\{0,1\}^{t^{\prime}}$. Both parties run a $\mathrm{OT}_{t^{\prime}}^{n}$ protocol on these randomly chosen values, where $\mathcal{S}$ inputs the pairs $\left\langle m_{i}^{0}, m_{i}^{1}\right\rangle$ and $\mathcal{C}$ inputs $r_{i}$ and obtains the masks $m_{i}=m_{i}^{r_{i}}$ as output. In the online phase, for each $i=1, \ldots, n, \mathcal{C}$ masks its input bits $b_{i}$ with $r_{i}$ as $\bar{b}_{i}=b_{i} \oplus r_{i}$ and sends these masked bits to $\mathcal{S}$. $\mathcal{S}$ responds with the masked pair of $t^{\prime}$-bit strings $\left\langle\bar{s}_{i}^{0}, \bar{s}_{i}^{1}\right\rangle=\left\langle m_{i}^{0} \oplus s_{i}^{0}, m_{i}^{1} \oplus s_{i}^{1}\right\rangle$ if $\bar{b}_{i}=0$ or $\left\langle\bar{s}_{i}^{0}, \bar{s}_{i}^{1}\right\rangle=\left\langle m_{i}^{0} \oplus s_{i}^{1}, m_{i}^{1} \oplus s_{i}^{0}\right\rangle$ otherwise. $\mathcal{C}$ obtains $\left\langle\bar{s}_{i}^{0}, \bar{s}_{i}^{1}\right\rangle$ and decrypts $s_{i}^{b_{i}}=\bar{s}_{i}^{r_{i}} \oplus m_{i}$. Overall, the online phase consists of two messages of size $n$ bits and $2 n t^{\prime}$ bits and negligible computation (XOR of bitstrings).

\subsubsection{OT Complexity}

Combining the previously described improvements for pre-computing and extending OT with the efficient OT protocol of [NP01] yields a highly efficient implementation of $\mathrm{OT}_{t}^{n}$ in the $\mathrm{RO}$ model as summarized in Table 2.5. Similarly, an efficient implementation in the standard model using correlation robust hashing can be obtained by combining with the protocol of [AIR01] instead.

\subsection{GC Protocols}

In this section we show how Garbled Circuits (GCs) are used in several protocols for secure computation in the two-party (\$2.3.1) and multi-party $(2.3 .2)$ setting. Further applications of GCs such as One-Time Programs (OTPs) (\$4.2) or verifiable computing (\$4.3) are described later in this thesis. 
Table 2.5: Complexity of $\mathrm{OT}_{t}^{n}$ in the Random Oracle Model

\begin{tabular}{|c|c|c|c|}
\hline \multicolumn{2}{|l|}{ Complexity } & Setup Phase & Online Phase \\
\hline \multicolumn{4}{|c|}{ For $n \leq t:$ [Bea95]+[NP01] } \\
\hline \multirow[t]{2}{*}{ Communication } & Moves & 3 & 2 \\
\hline & Data [bits] & $6 n t$ & $2 n t$ \\
\hline \multirow[t]{2}{*}{ Computation Client $\mathcal{C}$} & $\mathrm{H}$ & $n$ & \\
\hline & EC mult & $2 n$ & \\
\hline \multirow[t]{2}{*}{ Server $\mathcal{S}$} & $\overline{\mathrm{H}}$ & $2 n$ & \\
\hline & EC mult & $2 n+1$ & \\
\hline \multicolumn{4}{|c|}{ For $n>t:$ [Bea95 + [IKNP03 + [NP01 } \\
\hline \multirow[t]{2}{*}{ Communication } & Moves & 4 & 2 \\
\hline & Data [bits] & $4 n t+6 t^{2}$ & $2 n t$ \\
\hline \multirow{4}{*}{ Computation Client $\mathcal{C}$} & $\mathrm{H}$ & $n+2 t$ & \\
\hline & EC mult & $2 t+1$ & \\
\hline & $\overline{\mathrm{H}}$ & $2 n+t$ & \\
\hline & EC mult & $2 t$ & \\
\hline
\end{tabular}

\subsubsection{Two-party Secure Function Evaluation (SFE)}

SFE allows two parties to implement a joint computation without using a Trusted Third Party (TTP), One classical example is the millionaires' problem [Yao86] where two millionaires want to know who is richer, without any of them revealing their net worth to the other or a TTP.

More formally, SFE is a cryptographic protocol that allows two players, client $\mathcal{C}$ with private input $\operatorname{in}_{\mathcal{C}}$ and server $\mathcal{S}$ with private input $\operatorname{in}_{\mathcal{S}}$, to evaluate a function $f$ on their private inputs:

$$
\left(\text { out }_{\mathcal{C}}, \text { out }_{\mathcal{S}}\right)=f\left(\text { in }_{\mathcal{C}}, \text { in }_{\mathcal{S}}\right) .
$$

The SFE protocol ensures that both parties learn only their respective output, i.e., $\mathcal{C}$ learns out ${ }_{\mathcal{C}}$ and $\mathcal{S}$ learns out ${ }_{\mathcal{S}}$, but nothing else about the other party's private input. In SFE, the function $f$ is known to both parties.4

Intuitively, according to the real/ideal world paradigm (e.g., Can01]), a SFE protocol executed in the real world is secure if and only if an adversary with defined capabilities can do no more harm to the protocol executed in the real world than in an ideal world where each party submits its input to a TTP which computes the results according to Equation (2.9) and returns them to the respective party.

In $\$ 2.3 .1 .1$ we start with the description of the classical SFE protocol of Yao [Yao86]

\footnotetext{
${ }^{4}$ If needed, SFE can be extended s.t. the function is known to only one of the parties and hidden
} from the other as described in $\$ 2.3 .1 .3$. 
which is secure against semi-honest adversaries and summarize how this protocol can be secured against more powerful covert and malicious adversaries in \$2.3.1.2. Afterwards, we show how the evaluated function itself can be hidden in $\$ 2.3 .1 .3$.

\subsubsection{SFE with Semi-honest Adversaries (Yao's Protocol)}

Yao's protocol [Yao86, LP09a, KSS10] for SFE of a function $f$ represented as boolean circuit (cf. 2.1.3.1) works as follows:

1. Create $G C$ : In the setup phase, the constructor (server $\mathcal{S}$ ) generates a $\mathrm{GC} \tilde{f}$ using algorithm createGC as described in $\$ 2.2 .2$ and sends $\tilde{f}$ to the evaluator (client $\mathcal{C}$ ).

2. Encrypt Inputs: Afterwards, in the online phase, the inputs of the two parties $\operatorname{in}_{\mathcal{C}}$, in $_{\mathcal{S}}$ are converted into the corresponding garbled input $\widetilde{\text { in }}=\left\{\widetilde{\text { in }}_{\mathcal{C}}, \widetilde{i n}_{\mathcal{S}}\right\}$ provided to $\mathcal{C}$ : For $\mathcal{S}$ 's inputs in $\mathcal{S}_{\mathcal{S}} \mathcal{S}$ simply sends the garbled values corresponding to his inputs to $\mathcal{C}$, i.e., $\widetilde{\mathrm{in}}_{\mathcal{S}, i}=\widetilde{\mathrm{in}}_{\mathcal{S}, i}$. Similarly, $\mathcal{C}$ must obtain the garbled values $\widetilde{\mathrm{in}}_{\mathcal{C}, i}$ corresponding to her inputs $\widetilde{\mathrm{in}}_{\mathcal{C}, i}$, but without $\mathcal{S}$ learning $\operatorname{in}_{\mathcal{C}, i}$. This can be achieved by running (in parallel for each bit $\operatorname{in}_{\mathcal{C}, i}$ of $\mathrm{in}_{\mathcal{C}}$ ) a 1-out-of-2 Oblivious Transfer (OT) protocol as described in $\$ 2.2 .3$.

3. Evaluate Function Under Encryption: Now, $\mathcal{C}$ can evaluate the GC $\tilde{f}$ on the garbled inputs in using algorithm evalGC as described in $\$ 2.2 .2$ and obtains the garbled outputs out $=\left\{{\widetilde{\text { out }_{\mathcal{C}}}}, \widetilde{\text { out }_{\mathcal{S}}}\right\}$.

4. Decrypt Outputs: Finally, the garbled outputs are converted into plain outputs for the respective party: For $\mathcal{C}$ 's outputs out $\mathcal{C}, \mathcal{S}$ reveals their permutation bits to $\mathcal{C}$ (this can be done already in the setup phase). For $\mathcal{S}$ 's outputs $\widetilde{o u t}_{\mathcal{S}}, \mathcal{C}$ sends the obtained permutation bits to $\mathcal{S}$.

Security. As proven in detail in [LP09b], Yao's protocol is secure against semi-honest adversaries.

We observe that in Yao's protocol the security of GC constructor $\mathcal{S}$ is computational as $\mathrm{GC}$ evaluator $\mathcal{C}$ can break the $\mathrm{GC}$ by guessing garbled input values, verify if they decrypt correctly and match them with the garbled inputs provided by $\mathcal{S}$. When instantiating $\mathrm{OT}$ with a protocol which provides statistical security for receiver $\mathcal{C}$ (e.g., using the $\mathrm{OT}$ protocol of [NP01]), the security of $\mathrm{GC}$ evaluator $\mathcal{C}$ is statistical.

Efficiency. The efficiency of Yao's protocol is dominated by the efficiency of the GC construction and OT for each input bit of $\mathcal{C}$.

As described in $\$ 2.2 .3$, OT requires only a constant number of public-key operations and allows to shift most communication and computation into the setup phase. The 
resulting setup phase requires to pre-compute $\mid$ in $_{\mathcal{C}} \mid \mathrm{OTs}$ (cf. \$2.2.3.4), create the GC $\tilde{f}$ (cf. $\$ 2.2 .2 .4$ ), and transfer $\tilde{f}$ to $\mathcal{C}$ (cf. Table 2.4).

The online phase is highly efficient as it requires only symmetric-key operations for evaluating $\widetilde{f}$ (cf. $\$ 2.2 .2 .4$ ), and three moves (two for the online phase of pre-computed $\mathrm{OT}$ and one for sending the output to $\mathcal{S})$ with about $t\left(2\left|\mathrm{in}_{\mathcal{C}}\right|+\left|\mathrm{in}_{\mathcal{S}}\right|\right)+\mid$ out $_{\mathcal{S}} \mid$ bits of communication in total.

\subsubsection{SFE with Stronger Adversaries}

GC-based SFE protocols can easily be protected against covert or malicious client $\mathcal{C}$ by using an $\mathrm{OT}$ protocol with corresponding security properties.

Efficient SFE protocols based on GCs which additionally protect against covert [AL07, GMS08] or malicious [LP07] server $\mathcal{S}$ rely on the following cut-and-choose technique: $\mathcal{S}$ creates multiple GCs, deterministically derived from random seeds $s_{i}$, and commits to each, e.g., by sending $\widetilde{f}_{i}$ or Hash $\left(\widetilde{f}_{i}\right)$ to $\mathcal{C}$. In the covert case, $\mathcal{C}$ asks $\mathcal{S}$ to open all but one $\mathrm{GC} \widetilde{f}_{I}$ by revealing the corresponding seeds $s_{i \neq I}$. For all opened functions, $\mathcal{C}$ computes $f_{i}$ and checks that they match the commitments. The malicious case is similar, but $\mathcal{C}$ asks $\mathcal{S}$ to open half of the functions, evaluates the remaining ones and chooses the majority of their results. Additionally, it must be guaranteed that $\mathcal{S}$ 's input into $\mathrm{OT}$ is consistent with the $\mathrm{GCs}$ as pointed out in [KS06], e.g., using committed or committing OT. The most recent construction of [LP10] improves over previous protocols (smaller number of GCs, completely removing the commitments, and also removing the need to increase the size of the inputs) by using a new primitive called cut-and-choose OT, an extension of parallel 1-out-of-2 OT with a cut-and-choose functionality.

The practical performance of cut-and-choose-based GC protocols has been investigated experimentally in [LPS08, [PSSW09]: Secure evaluation of the AES functionality (a boolean circuit with 33, 880 gates) between two Intel Core 2 Duo's running at $3.0 \mathrm{GHz}$, with $4 \mathrm{~GB}$ of RAM connected by gigabit ethernet takes approximately $0.5 \mathrm{MB}$ data transfer and $7 \mathrm{~s}$ for semi-honest, 8.7 MB / 1 min for covert, and $400 \mathrm{MB} / 19 \mathrm{~min}$ for malicious adversaries [PSSW09]. This shows that protecting GC protocols against stronger adversaries comes at a relatively high prize.

For completeness, note that cut-and-choose may be avoided with SFE schemes such as [JS07] which prove in zero-knowledge that the GC was computed correctly and the inputs are consistent with committed inputs [GMY04]. However, their elementary steps involve public-key operations. As estimated in [PSSW09], such protocols which apply public-key operations per gate [JS07, NO09] often require substantially more computation than cut-and-choose-based protocols.

We further note that there are yet other approaches to malicious security such as the approach of [IPS08] which achieves malicious security by simulating a Secure Multi-Party Computation (SMPC) protocol inside a secure two-party computation protocol with semi-honest security. Their precise performance comparison is a desired 
but complicated undertaking, since there are several performance measures, and some schemes may work well only for certain classes of functions.

\subsubsection{SFE with Private Functions}

In some application scenarios of SFE, the evaluated function itself needs to be hidden, e.g., as it represents intellectual property of a service provider. This can be achieved by securely evaluating a Universal Circuit (UCi) which can be programmed to simulate any circuit $C$ and hence entirely hides $C$ (besides an upper bound on the number of inputs, number of gates and number of outputs). Efficient UCi constructions to simulate circuits consisting of up to $k$ two-input gates are given in [Val76, KS08b]. Generalized UCis of [SS08] can simulate circuits consisting of $d$-input gates. Which UCi construction is favorable depends on the size of the simulated functionality: Small circuits can be simulated with the UCi construction of [Sch08, SS08] with overhead $\mathcal{O}\left(k^{2}\right)$ gates, medium-size circuits benefit from the construction of [KS08b] with overhead $\mathcal{O}\left(k \log ^{2} k\right)$ gates and for very large circuits the construction of [Val76] with overhead $\mathcal{O}(k \log k)$ gates is most efficient. Explicit sizes and a detailed analysis of the breakeven points between these constructions are given in [SS08]. The alternative approach of [KM10] for evaluating private functions without using UCis has complexity linear in $k$, but requires $\mathcal{O}(k)$ public-key operations.

While UCis entirely hide the structure of the evaluated functionality $f$, it is sometimes sufficient to hide $f$ only within a class of topologically equivalent functionalities $\mathcal{F}$, called secure evaluation of a semi-private function $f \in \mathcal{F}$ [PSS09]. The circuits for many standard functionalities are topologically equivalent and differ only in the specific function tables, e.g., comparison $(<\rangle,,=, \ldots)$ or addition/subtraction as described later in 3.3 . When no cut-and-choose is used for GCs, it is possible to directly evaluate the circuit and avoid the overhead of a UCi for semi-private functions, as GC constructions of MNPS04 and [NPS99] (cf. \$2.2.2.3) completely hide the type of the gates from GC evaluator. These techniques were used for example in [FAL04, FAZ05, FLA06, FAL06, PSS09].

\subsubsection{Garbled Circuit Protocols with Multiple Parties}

Garbled Circuits (GCs) can also be used for SMPC, i.e., secure computation with more than two parties. In the following we describe applications of GCs to SMPC in 2.3.2.1 and secure mobile agents in $\$ 2.3 .2 .2$.

In the multi-party setting, one party, $\mathrm{GC}$ creator, which is assumed to behave correctly, creates the GC (cf. algorithm createGC in \$2.2.2.2); another party, GC evaluator, obliviously obtains the corresponding garbled inputs and evaluates the GC (cf. algorithm evalGC in $\$ 2.2 .2 .2$ ). The other parties provide inputs to or obtain outputs from the protocol. 
We will show later in $\$ 4$ that the GC creator can be implemented with constant-size memory, e.g., within a tamper-proof hardware (HW) token.

Verifiability of GCs. As discussed in detail in $\$ 4$, the GC evaluator, who evaluates the GC on the garbled inputs, needs not to be trusted at all. Indeed, GC evaluation can be performed by one or more untrusted parties as the garbled outputs allow to verify that the GC evaluation was done correctly [NPS99]: For each garbled output $\widetilde{z}_{i}$, the $\mathrm{GC}$ creator provides the output decryption information $\left\langle 0, G\left(\widetilde{z}_{i}^{0}\right)\right\rangle,\left\langle 1, G\left(\widetilde{z}_{i}^{1}\right)\right\rangle$, where $G$ is a one-way function (e.g., a cryptographic hash function). This allows to check whether $\widetilde{z}_{i}$ is correct, i.e., either $\widetilde{z}_{i}=\widetilde{z}_{i}^{0}$ or $\widetilde{z}_{i}=\widetilde{z}_{i}^{1}$, and which is the corresponding plain value without revealing the values $\widetilde{z}_{i}^{0}$ and $\widetilde{z}_{i}^{1}$. As the GC evaluator is unable to guess a correct $\widetilde{z}_{i}$ (except with negligible probability), she must have obtained it by honestly evaluating the $\mathrm{GC}$.

\subsubsection{Secure Multi-Party Computation with Two Servers}

As proposed in [NPS99], Yao's GC protocol (cf. \$2.3.1.1) can be turned into a Secure Multi-Party Computation (SMPC) protocol with multiple input players, multiple output players, and two non-colluding computation players who perform the secure computation: $\mathrm{GC}$ creator is trusted by the output players to behave semi-honestly and GC evaluator can even be malicious.

For multiple input players, the parallel 1-out-of-2 OT protocol (cf. 32.2 .3 ) is replaced with a parallel 1-out-of-2 proxy OT protocol. The proxy OT protocol splits the role of the chooser in the $\mathrm{OT}$ protocol into two parties: the chooser (input player) provides the secret input bit $b$, and the proxy (GC evaluator) learns the chosen output string $s^{b}$, but neither $b$ nor $s^{1-b}$. As described in [NPS99, Appendix A], efficient OT protocols (e.g., the protocols of [NP01, AIR01] described in \$2.2.3) can be naturally converted into a proxy OT protocol as follows: The chooser sends the two public keys, of which she knows the trapdoor to exactly one, to the sender. The sender applies an errorcorrecting code to each of the two strings $s^{0}, s^{1}$ and sends their encryptions under the respective public key to the proxy. The proxy uses the trapdoor obtained by the chooser to decrypt both ciphertexts obtained from the sender and uses the error correcting code to compute $s^{b}$.

For multiple output players, the GC evaluator forwards the garbled outputs to the respective output player who can decrypt and verify the correctness of the output using the output decryption information obtained from the $\mathrm{GC}$ creator.

\subsubsection{Secure Mobile Agents}

In the mobile agents scenario, the originator creates software agents that can perform tasks on behalf of the originator. After creating the agents for some specific purpose, the originator sends them out to visit various remote hosts, where the agents perform 
computations on behalf of the originator. When the agents return home, the originator retrieves the results of these computations from the agents. The utility of this paradigm is based on the ability of the originator to go offline after sending the agents out, and, ideally, no further interaction between the agent and the originator or the host should be required. A possible application would be an agent which travels through the web to select, depending on a policy of the originator, an offer for the most suited product at the lowest price.

Secure mobile agents extend the mobile agents scenario with security features. Here, the visited hosts are not trusted by the originator and the other way around. When an agent visits a host, it carries along some state from previous computations and uses this together with input from the host to compute the new agent state possibly along with an output provided to the host. The agent state (both old and new) is "owned" by the agent, and should be protected from potentially malicious hosts, whereas the host input and output are "owned" by the host and should likewise be protected from potentially malicious agents. The code evaluated by the agent (policy) can be hidden as well by evaluating a UCi (cf. \$2.3.1.3).

The concept of secure mobile agents was introduced in [ST98] who give partial solutions based on Homomorphic Encryption (HE) 2.2.1). More practical constructions for secure mobile agents proposed afterwards are based on GCs. An agent can securely migrate from one host to the next by running a (slightly modified) GC-based SFE protocol (cf. \$2.3.1) between the two hosts as described in [CCKM00. To protect against malicious hosts, a TTP can be used to generate the GCs, similarly to the GC constructor in the construction of [NPS99] (cf. \$2.3.2.1), as proposed in [ACCK01]. The assumption of the TTP was later on removed in [TX03a] and a construction which achieves universal composability is given in [XT04]. Finally, non-interactive OT based on trusted $\mathrm{HW}$ removes the communication overhead to the essential minimum where the agent is sent from one host to the next in a single message [GT08]. 
Chapter 2 Secure Function Evaluation Basics 


\section{Chapter 3}

\section{Circuit Optimizations and Constructions}

The complexity of many cryptographic protocols, including today's most efficient Secure Function Evaluation (SFE) protocols, depends linearly on the size of the boolean circuit representation of the function to be evaluated. In this chapter we show how to optimize boolean circuits and give efficient circuit constructions.

\subsection{Motivation}

Outline. In the beginning of this chapter we motivate protocols that allow "free" evaluation of XOR gates (\$3.1.1), related work (\$3.1.2), and preliminaries and notation used in this chapter $(3.1 .3)$.

Afterwards, we give optimization algorithms that reduce the size of boolean circuits in particular when XOR gates can be evaluated "for free" in $\$ 3.2$.

In $\$ 3.3$ we show how our optimizations allow to construct more efficient circuits for standard functionalities such as integer comparison. We also give efficient circuit constructions for computing minima (or maxima) and for fast multiplication.

As applications we consider efficient protocols for secure comparison and first-price auctions in 3.4 .

\subsubsection{Protocols with Free XOR}

The following cryptographic protocols allow to evaluate XOR gates (addition modulo 2) at a substantially lower cost than non-XOR gates such as AND gates (multiplication modulo 2). All these applications benefit from our circuit optimizations of \$3.2.2 which reduce the number of costly non-XOR gates.

Zero-Knowledge. Zero-knowledge interactive proofs (ZKIP) for SAT, i.e., to prove satisfiability of a formula represented as boolean circuit in zero-knowledge, can be constructed based on the quadratic residuosity assumption [BC86]. This protocol allows 
non-interactive processing of NOT and XOR gates and requires one round of interaction for other gates. Extensions of these proof techniques of [BBP91, BLP93, BBP95, BP96, BDP00 inherit and rely on the property of cheap non-interactive processing of $\mathrm{XOR}$ gates.

Secure Function Evaluation (SFE), Gate evaluation secret sharing [Kol05] allows information theoretically secure $\mathrm{SFE}$ where the size of shares remains constant for $\mathrm{XOR}$ gates and increases (doubles) for non-XOR gates. Also the Garbled Circuit (GC) construction of [KS08a] provides "free", i.e., non-interactive and computationally inexpensive, garbled XOR gates (cf. \$2.2.2.3).

Homomorphic Encryption (HE), The homomorphic cryptosystems of [BGN05, GHV10] allow to evaluate 2-DNF formulas, i.e., polynomially many additions and one multiplication under encryption (cf. $\$ 2.2 .1 .2$ ). When instantiated over $\mathbb{F}_{2}$, such schemes allow to evaluate many XOR gates but only one AND gate.

Recent fully HE systems [Gen09a, Gen09b, SV10, DGHV10, SS10b] support evaluation of arbitrarily many additions and multiplications under encryption (cf. \$2.2.1.2). However, addition (evaluation of an XOR gate) is more efficient than multiplication (evaluation of an AND gate) as it has less impact on the "error" of the ciphertext and hence less "refresh" (re-encrypt) operations are needed (cf. [Gen09a]).

\subsubsection{Related Work}

Existing frameworks for automatic generation of SFE protocols MNPS04, BDNP08, YLP06 allow to specify the function to be computed in a function description language and automatically transform this description into a functionally equivalent boolean circuit. Alternatively, existing tools from electronic design automation such as compilers for Very high speed integrated circuit Hardware Description Language (VHDL) or Verilog could be used for generating boolean circuits. The open-source Fairplay compiler suite MNPS04, BDNP08 performs optimizations on the high-level function description language, e.g., using techniques such as peek-hole optimization, duplicate code removal, or dead code elimination. Our optimizations are on the lower abstraction level of circuits and can also be applied to further optimize the output of circuits generated with the Fairplay compiler (e.g., as used in [PSSW09, Sect. 3] to minimize the AES circuit).

A special case of our algorithm for propagating 1-input gates into their predecessor or successor gates was mentioned for NOT gates in YLP05]. Further, they give explicit circuit sizes for standard functionalities such as addition, subtraction, multiplication, multiplexer, and comparison of unsigned integers when circuits are decomposed into 2-input gates. However, when XOR gates are not "for free", the case they consider, $\mathrm{GC}$ constructions are more efficient when not decomposing 3-input gates into more than two 2-input gates [LPS08]. 
Gates with many inputs can be decomposed into smaller gates using Shannon's expansion theorem [Sha49] which replaces a $(d+1)$-input gate by two $d$-input gates and one bit-multiplexer (one 3-input gate or three 2-input gates). For decomposition into 2-input gates, the Quine-McCluskey algorithm can be extended to also handle XOR gates [Tur96]. However, these algorithms use a monotonic cost metric for optimization which does not consider "free" XOR gates.

For optimizations in the presence of "free" XOR gates, Boyar et al. BP96, BPP00, BDP00 considered multiplicative complexity ${ }^{1}$ of symmetric functions, i.e., functions that only dependent on the hamming weight of their inputs and hence the inputs of such functions can be permuted arbitrarily. As a corollary, Boyar et al. describe an efficient instantiation for full adders (cf. \$3.3.1.1). In [BP06] they show that the multiplicative complexity for computing the Hamming weight of an $n$-bit string is exactly $n-H^{\mathbb{N}}(n)$ for $n \geq 1$, where $H^{\mathbb{N}}(n)$ is the Hamming weight of $n$. Recently, Boyar and Peralta [BP10] proposed a heuristic for optimizing the multiplicative complexity of circuits in a two-step process. The first step reduces the non-linearity, i.e., the number of AND gates, of the circuit; the second step reduces the number of XOR gates in the linear component. This optimization technique is used to construct a compact S-box for the Advanced Encryption Standard (AES) [NIS01] with 32 AND gates and $83 \mathrm{X}(\mathrm{N}) \mathrm{OR}$ gates [BP10].

\subsubsection{Preliminaries and Notation}

Recall, a $d$-input gate is a boolean function which maps from $d$ input bits to one output bit (cf. Definition 2).

Notation 1 (d-input gate). We write gates as $\left(\mathrm{in}_{1}, \ldots, \mathrm{in}_{d}\right)\left[T_{d}\right]$ consisting of the list of inputs $\mathrm{in}_{1}, \ldots, \mathrm{in}_{d}$ and the gate table $T_{d}$, a string of $2^{d}$ bits. The $i$-th element of $T_{d}$ is equal to the value of the function evaluated by the gate $G_{d}\left(\mathrm{in}_{1}, \ldots, \mathrm{in}_{d}\right)$ for $i=\sum_{j=1}^{d} 2^{j-1} \mathrm{in}_{j}$. We write 0 as shortcut for the constant (0-input) gate ()[0] and analogously 1 for ()[1].

Example 1. The gate $(a, b)[0010]$ computes the function $a, b \mapsto(\neg a) \wedge b$.

Definition 3 (Class and number of $d$-input gates). We denote the class of d-input gates with $\mathcal{G}_{d}:=\left\{\left(\mathrm{in}_{1}, \ldots, \mathrm{in}_{d}\right)\left[T_{d}\right]: T_{d} \in\{0,1\}^{2^{d}}\right\}$. The number of $d$-input gates is

$$
\# \mathcal{G}_{d}=2^{2^{d}}
$$

Example 2. The class of two-input gates is $\mathcal{G}_{2}=\left\{(a, b)\left[T_{2}\right]: T_{2} \in\{0,1\}^{4}\right\}$ and there exist $\# \mathcal{G}_{2}=2^{2^{2}}=16$ two-input gates.

\footnotetext{
${ }^{1}$ Multiplicative complexity of a function measures the number of AND gates in its circuit (and gives NOT and XOR gates for free).
} 
Definition 4 (Trivial $d$-input gates). A trivial d-input gate $G_{d}$ can be expressed as a wire or an equivalent gate with $d^{\prime}<d$ inputs. A non-trivial gate is not trivial. The class of d-input gates can be partitioned into the class of trivial d-input gates $\mathcal{G}_{d}^{\text {triv }}$ and the class of non-trivial d-input gates $\mathcal{G}_{d}^{\text {nontriv }}$, i.e., $\mathcal{G}_{d}=\mathcal{G}_{d}^{\text {triv }} \uplus \mathcal{G}_{d}^{\text {nontriv }}$. From this we have

$$
\# \mathcal{G}_{d}=\# \mathcal{G}_{d}^{\text {triv }}+\# \mathcal{G}_{d}^{\text {nontriv }}
$$

Notation 2 (Costs of boolean gates and circuits). We denote the costs of (non-trivial) $d$-input gates with $\left|\mathcal{G}_{d}\right|$. Similarly, the costs of a boolean circuit $C$, denoted as $|C|$, are the sum of the costs of its gates.

Example 3. In the GC construction of [MNPS04] the garbled table of a non-trivial $d$-input gate has size $\left|\mathcal{G}_{d}\right|=2^{d} \cdot(t+1)$ bits (cf. \$2.2.2).

Assumption 1 (Monotonic costs of non-trivial gates). We assume that the costs of non-trivial gates are monotonic, i.e., non-trivial d-input gates have at most the same cost as non-trivial $(d+1)$-input gates: $\left|\mathcal{G}_{d}\right| \leq\left|\mathcal{G}_{d+1}\right|$.

This assumption is fulfilled for the computation and communication costs of all GC constructions presented in $\$ 2.2 .2$.

\subsection{Circuit Optimizations}

Publication Info: Parts of the following results are published in [PSS09, Sect. 8] (\$3.2.1) and [PSSW09, Sect. 3] (\$3.2.2).

\subsubsection{Minimizing Circuits}

In the following we show how the costs of a given boolean circuit can be minimized. Under Assumption 1, the costs of a boolean circuit can be minimized by 1) replacing trivial gates with wires or an equivalent gate with less inputs, or 2) removing gates.

We concentrate on gates with a small number of inputs, i.e., $d \in\{0,1,2,3\}$, as these are the gates from which circuit building blocks for fundamental functionalities such as operations on integer values are composed (cf. \$3.3).

\subsubsection{Replacing Trivial Gates}

Trivial gates can be replaced with a wire or a gate with less inputs using the replacement rules shown in Fig. 3.1: The first column $(\mathrm{T})$ of the replacement tables contains the gate table and the second column (To) contains the functionally equivalent replacement in postfix notation. For example the gate $(a, b, c)[T]$ with gate table $T=00001010$ represents the function $(\neg a) \wedge c$ and hence can be replaced by the 


\begin{tabular}{|c|c|c|c|c|c|c|c|c|}
\hline \multicolumn{2}{|c|}{$d=0:()[\mathbf{T}]$} & \multicolumn{3}{|c|}{$d=1:(\mathbf{a})[\mathbf{T}]$} & \multicolumn{4}{|c|}{$d=2:(\mathbf{a}, \mathbf{b})[\mathbf{T}]$} \\
\hline $\mathrm{T}$ & To & $\mathrm{T}$ & To & & $\mathrm{T}$ & To & $\mathrm{T}$ & To \\
\hline 0 & - & 00 & 0 & & 0000 & 0 & 1111 & 1 \\
\hline 1 & - & 01 & $\mathrm{a}$ & & 0011 & $\mathrm{~b}$ & 1100 & (b) $[10]$ \\
\hline & & 11 & 1 & & 0101 & a & 1010 & (a) $[10]$ \\
\hline & & & & $d=3:$ & $a, b, c)[T]$ & & & \\
\hline & $\mathrm{T}$ & To & & $\mathrm{T}$ & To & & $\mathrm{T}$ & To \\
\hline & 00000000 & 0 & & 01000100 & $(a, b)[0100]$ & & 1111111 & 1 \\
\hline & 00000011 & $(b, c)[00$ & & 01010000 & $(\mathrm{a}, \mathrm{c})[0100]$ & & 1111100 & $(b, c)[1110]$ \\
\hline & 00000101 & $(\mathrm{a}, \mathrm{c})[00$ & & 01010101 & a & & 1111010 & $(\mathrm{a}, \mathrm{c})[1110]$ \\
\hline & 00001010 & $(\mathrm{a}, \mathrm{c})[00$ & & 01011010 & $(\mathrm{a}, \mathrm{c})[0110]$ & & 1110101 & $(\mathrm{a}, \mathrm{c})[1101]$ \\
\hline & 00001100 & $(\mathrm{~b}, \mathrm{c})[00$ & & 01011111 & $(\mathrm{a}, \mathrm{c})[0111]$ & & 1110011 & $(\mathrm{~b}, \mathrm{c})[1101]$ \\
\hline & 00001111 & c & & 01100110 & $(a, b)[0110]$ & & 1110000 & (c) $[10]$ \\
\hline & 00010001 & $(\mathrm{a}, \mathrm{b})[0 \mathrm{c}$ & & 01110111 & $(a, b)[0111]$ & & 1101110 & $(\mathrm{a}, \mathrm{b})[1110]$ \\
\hline & 00100010 & $(a, b)[00$ & & 10001000 & $(a, b)[1000]$ & & 1011101 & $(\mathrm{a}, \mathrm{b})[1101]$ \\
\hline & 00110000 & $(\mathrm{~b}, \mathrm{c})[01$ & & 10011001 & $(a, b)[1001]$ & & 1001111 & $(b, c)[1011]$ \\
\hline & 00110011 & $\mathrm{~b}$ & & 10100000 & $(\mathrm{a}, \mathrm{c})[1000]$ & & 1001100 & (b) $[10]$ \\
\hline & 00111100 & $(b, c)[01$ & & 10100101 & $(\mathrm{a}, \mathrm{c})[1001]$ & & 1000011 & $(\mathrm{~b}, \mathrm{c})[1001]$ \\
\hline & 00111111 & $(b, c)[01$ & & 10101010 & (a) $[10]$ & & 1000000 & $(\mathrm{~b}, \mathrm{c})[1000]$ \\
\hline & & & & 10101111 & $(\mathrm{a}, \mathrm{c})[1011]$ & & & \\
\hline & & & & 10111011 & $(a, b)[1011]$ & & & \\
\hline
\end{tabular}

Figure 3.1: Replacement of Trivial $d$-input Gates

functionally equivalent 2-input gate $(a, c)[0010]$. Non-trivial gates which cannot be replaced are not listed or marked with - .

The following theorem describes how many of the $d$-input gates are trivial and hence can be replaced by such rules:

Theorem 1 (Number of trivial $d$-input gates).

$$
\# \mathcal{G}_{d}^{\text {triv }}= \begin{cases}0 & \text { for } d=0 \\
d+\sum_{i=0}^{d-1}\left(\begin{array}{l}
d \\
i
\end{array}\right) \# \mathcal{G}_{i}^{\text {nontriv }} & \text { for } d>0\end{cases}
$$

Proof of Theorem 1. As both constant gates are non-trivial we have $\# \mathcal{G}_{0}^{\text {triv }}=0$. For $d$-input gates we have as first $d$ trivial gates the wires $\mathrm{in}_{1}, \ldots, \mathrm{in}_{d}$. Additionally, for each subset $S_{i}$ of $0 \leq i<d$ inputs, each non-trivial $i$-input gate with inputs $S_{i}$ can be augmented into a trivial $d$-input gate $G_{d}$ where all inputs of $G_{d}$ which are not in $S_{i}$ are ignored. 
Using equations (3.1), (3.2) and (3.3), the number of trivial and non-trivial $d$-input gates can be computed efficiently via dynamic programming. As shown in Table 3.1 . the fraction of trivial gates decreases rapidly with $d$, so for increasing $d$ most gates are non-trivial and cannot be replaced.

Table 3.1: Number of Trivial $d$-input Gates

\begin{tabular}{c||c|c|c|c}
$d$ & $\# \mathcal{G}_{d}^{\text {nontriv }}$ & $\# \mathcal{G}_{d}^{\text {triv }}$ & $\# \mathcal{G}_{d}$ & $\# \mathcal{G}_{d}^{\text {triv }} / \# \mathcal{G}_{d}$ \\
\hline 0 & 2 & 0 & 2 & $(0 \%)$ \\
1 & 1 & 3 & 4 & $75 \%$ \\
2 & 10 & 6 & 16 & $37.5 \%$ \\
3 & 218 & 38 & 256 & $14.8 \%$ \\
4 & 64,594 & 942 & 65,536 & $1.44 \%$
\end{tabular}

\subsubsection{Removing Gates}

To further reduce the circuit size, we describe an optimization algorithm that tries to eliminate constant gates (i.e., 0-input gates) and 1-input gates in a circuit $C$. The algorithm reduces the number of gates respectively their degree. Besides the well known propagation of constant gates (Step 1), our algorithm additionally eliminates resulting gates with one input by incorporating them into surrounding gates (Step 2 and Step 3), which results in a smaller circuit size.

Terminology. As described in $\$ 2.1 .3 .1$, we assume that the gates within a circuit are ordered topologically. An output gate is a gate whose output is also an output of $C$. Similarly, an input gate is a gate, which has at least one input that is also an input of $C$. For gate $G_{i}$, $\operatorname{pred}\left(G_{i}\right)$ denotes the set of its predecessors, i.e., gates whose output is an input into $G_{i}$. Analogously, $\operatorname{succ}\left(G_{i}\right)$ denotes the set of $G_{i}$ 's successors, i.e., gates having the output of $G_{i}$ as input. The fan-out of a gate $G_{i}$ is the number of its successors, i.e., fanout $\left(G_{i}\right)=\# \operatorname{succ}\left(G_{i}\right)$.

Optimization. Algorithm 1 optimizes a circuit $C$ by removing constant and 1-input gates as described in the following:

Step 1: Eliminate constant gates. The first step of Algorithm 1 eliminates all constant gates $G_{j}=()\left[v_{j}\right]$ of circuit $C$ with respective constant value $v_{j} \in\{0,1\}$. For all gates $G_{i}$ with degree $d_{i}$ having the output of $G_{j}$ as $k_{i}$-th input, the function eliminateConstInput $\left(G_{i}, k_{i}, v_{j}\right)$ is invoked that eliminates the corresponding input of $G_{i}$. Only the lines of the function table of $G_{i}$ with value $v_{j}$ in the $k_{i}$-th position are used while the other entries are eliminated, i.e., the modified gate $G_{i}^{\prime}$ 


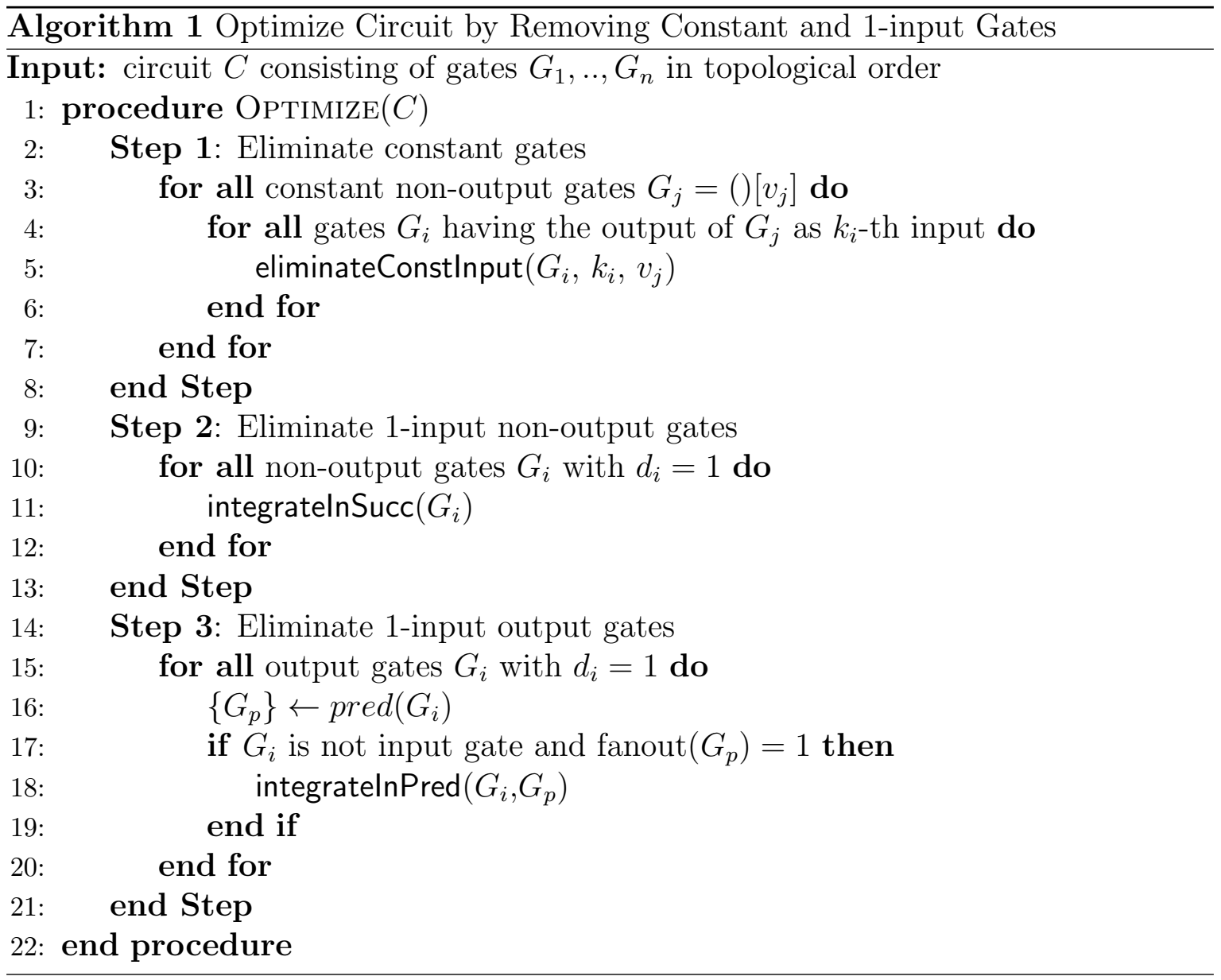

computes $G_{i}^{\prime}\left(\operatorname{in}_{1}, . ., \operatorname{in}_{k_{i}-1}, \operatorname{in}_{k_{i}+1}, . ., \operatorname{in}_{d_{i}}\right)=G_{i}\left(\operatorname{in}_{1}, . ., \operatorname{in}_{k_{i}-1}, v_{j}, \operatorname{in}_{k_{i}+1}, . ., \operatorname{in}_{d_{i}}\right)$. For an efficient implementation of Algorithm 1 it is crucial that eliminateConstInput() does not copy the entire function table of a gate $G_{i}$ with degree $d_{i}$ for each elimination of a constant input as this would result in runtime $\mathcal{O}\left(\# c_{i} \cdot 2^{d_{i}}\right)$ for each gate. Instead, the constant gates are marked in runtime $\mathcal{O}\left(\# c_{i}\right)$ and afterwards all constant gates are eliminated simultaneously in runtime $\mathcal{O}\left(2^{d_{i}}\right)$ by copying the corresponding elements of the function table. This results in runtime $\mathcal{O}\left(2^{d_{i}}\right)$ per gate. Resulting constant gates $G_{i}^{\prime}$ with $d_{i}^{\prime}=0$ are propagated into their successors by recursively calling eliminateConstlnput $\left(G_{s}, k_{s}, G_{i}\left(v_{j}\right)\right)$ for all $G_{s} \in \operatorname{succ}\left(G_{i}^{\prime}\right)$ having $G_{i}^{\prime}$ as $k_{s}$-th input. If the constant gate $G_{i}^{\prime}$ is not an output gate it is eliminated afterwards.

After termination of Step 1 there might be 1-input gates $G_{i}$ left. The next two steps of Algorithm 1 try to remove these by incorporating their functionalities into their successors (Step 2) or predecessors (Step 3).

Step 2: Eliminate non-output gates with one input. The second step of Al- 
gorithm 1 eliminates 1 -input gates $G_{i}$ that are not output gates. The functionality of $G_{i}$ is incorporated into its successors $G_{s} \in \operatorname{succ}\left(G_{i}\right)$ by invoking the function integratelnSucc $\left(G_{i}\right)$. This function eliminates $G_{i}$ by replacing it with a wire and incorporating the functionality of $G_{i}$ into the function tables of all its successors $G_{s} \in \operatorname{succ}\left(G_{i}\right)$ : Let the output of $G_{i}$ be the $k$-th input of $G_{s}$ and $d$ the degree of $G_{s}$. Then, the modified gate $G_{s}^{\prime}$ computes $G_{s}^{\prime}\left(\operatorname{in}_{1}, . ., \operatorname{in}_{k}, . ., \mathrm{in}_{d}\right)=G_{s}\left(\mathrm{in}_{1}, . ., G_{i}\left(\mathrm{in}_{k}\right), . ., \mathrm{in}_{d}\right)$. Note that, independent of the functionality $G_{i}$, the resulting gate $G_{s}^{\prime}$ has the same number of inputs $d$ as $G_{s}$ but additionally incorporates the functionality of $G_{i}$. As in Step 1, for runtime $\mathcal{O}\left(2^{d_{i}}\right)$ per gate the modifications of the function tables are not applied directly but first marked and then done simultaneously.

Step 3: Eliminate output gates with one input. The third step of Algorithm 1 tries to eliminate 1-input gates $G_{i}$ that are output gates. The functionality of $G_{i}$ is incorporated into its predecessor $G_{p}$, if and only if $G_{i}$ is the only successor of $G_{p}$, i.e., fanout $\left(G_{p}\right)=1$. In this case, the function integratelnPred $\left(G_{i}, G_{p}\right)$ is invoked which eliminates gate $G_{i}$ by replacing it with a wire and incorporates its functionality into gate $G_{p}$ with $d$ inputs. The modified gate $G_{p}^{\prime}$ computes $G_{p}^{\prime}\left(\operatorname{in}_{1}, . ., \mathrm{in}_{d}\right)=G_{i}\left(G_{p}\left(\operatorname{in}_{1}, . ., \operatorname{in}_{d}\right)\right)$. As in Step 2, this optimization step is independent of the functionality $G_{i}$ and the resulting gate $G_{p}^{\prime}$ has the same degree as $G_{p}$ but additionally incorporates the functionality of $G_{i}$.

The following theorem summarizes the correctness and efficiency of Algorithm 1 .

Theorem 2. Algorithm 1 efficiently eliminates all $d \in\{0,1\}$-input gates that are not output gates of circuit $C$ in runtime $\mathcal{O}(|C|)$. The optimized circuit $C^{\prime}$ has at most the same size and computes the same functionality as $C$.

Proof. Let $n$ denote the number of gates of $C$ in the following.

1. Termination: Algorithm 1 always terminates as all loops are upper bounded and the recursive call of eliminateConstInput() in Step 1 terminates if $G_{i}$ has no successors.

2. Efficiency: Step 1 of Algorithm 1 first marks all constant gates in runtime $\mathcal{O}(|C|)$. Afterwards, the marked constant gates are eliminated in $\mathcal{O}(|C|)$. Step 2 also needs at most $\mathcal{O}(|C|)$ operations for elimination of gate $G_{i}$, marking and incorporating the functionality of $G_{i}$ into the succeeding gates, and analogously Step 3 runs in $\mathcal{O}(|C|)$ as well. Hence, the overall runtime of Algorithm 1 is in $\mathcal{O}(|C|)$.

3. All constant gates that are not outputs are eliminated: Step 1 of Algorithm 1 eliminates all constant gates that are not output gates by incorporating them into their successor gates $G_{i}$ in eliminateConstlnput(). 
4. All 1-input gates that are not outputs are eliminated: Step 2 of Algorithm 1 eliminates all 2-input gates that are not output gates by incorporating them into their successor gates $G_{i}$ in integratelnSucc().

5. Size is not increased: All steps do not increase the size of the circuit: $\left|C^{\prime}\right| \leq|C|$.

6. Functional equivalence: None of the optimizations performed in Algorithm 1 changes the functionality of $C$ as they incorporate the values of constant gates that are not output gates (Step 1) respectively 1-input gates (Step 2 and Step 3) into the functionality of surrounding gates. The functionality $f^{\prime}$ computed by the optimized circuit $C^{\prime}$ with $u$ inputs is identical to the functionality $f$ computed by the original circuit $C: \forall\left(\operatorname{in}_{1}, . ., \operatorname{in}_{u}\right) \in\{0,1\}^{u}: f^{\prime}\left(\operatorname{in}_{1}, . ., \mathrm{in}_{u}\right)=f\left(\mathrm{in}_{1}, . ., \mathrm{in}_{u}\right)$.

This concludes the proof of Theorem 2 .

\subsubsection{Minimizing Circuits with Free XOR}

As motivated in 3.1 .1 , some cryptographic constructions allow evaluation of XOR gates "for free". In the following we show how to optimize circuits when XOR gates have negligible costs compared to non-XOR gates. In this case it is beneficial to minimize the number of non-trivial non-XOR gates while potentially increasing the number of ("free") XOR gates.

Definition 5 (XOR-trivial $d$-input gates). An XOR-trivial d-input gate $G_{d}$ is either a trivial d-input gate ( $c f$. Definition (4), or it can be replaced by arbitrary many XOR gates and at most one gate with $d^{\prime}<d$ inputs. XOR-non-trivial gates are not XORtrivial.

When XOR gates are "for free", an XOR-trivial $d$ input gate has less costs than an XOR-non-trivial $d$-input gate: $\left|\mathcal{G}_{d}^{\text {XORtriv }}\right|<\left|\mathcal{G}_{d}^{\text {XORnontriv }}\right|$.

XOR-trivial 2-input gates. For $d=2$ we have as XOR-trivial gates the 6 trivial 2-input gates shown in Fig. 3.1, and additionally the XOR gate with gate table [0110] and the XNOR gate with gate table [1001] which can be replaced by an XOR gate and a NOT gate as $(a, b)[1001]=((a, b)[0110])[10]$. Overall, the number of XORtrivial 2-input gates is $\# \mathcal{G}_{2}^{\text {XORtriv }}=6+2=8$ and the fraction of XOR-trivial gates is $\# \mathcal{G}_{2}^{\text {XORtriv }} / \# \mathcal{G}_{2}=8 / 16=50 \%$.

XOR-trivial 3-input gates. In the following we show that also half of the 3-input gates are XOR-trivial and can be replaced by at most one 2-input non-XOR gate and at most three XOR gates.

It is easy to see that the optimal topology for replacing a non-trivial 3-input gate (written in postfix notation) is

$$
(((\lambda) X O R,(\mu) X O R)[\tau]), \nu) X O R
$$


where $\lambda, \mu, \nu \subseteq\{a, b, c\}$, and $\tau$ is the gate table of a 2-input gate. Based on this structure we computed by brute-force enumeration of all $2^{3} \cdot 2^{3} \cdot 2^{2^{2}} \cdot 2^{3}=8,192$ possibilities for $\lambda, \mu, \tau, \nu$ the optimal replacement for XOR-non-trivial 3-input gates.

The resulting 90 replacements for XOR-trivial 3-input gates which are not already listed in the replacement table of 38 trivial gates in Fig. 3.1 are given in Fig. 3.2 . Overall, the number of XOR-trivial 3-input gates is $\# \mathcal{G}_{3}^{\text {XORtriv }}=90+38=128$ and the fraction of XOR-trivial 3 -input gates is $\# \mathcal{G}_{3}^{\text {XORtriv }} / \# \mathcal{G}_{3}=128 / 256=50 \%$.

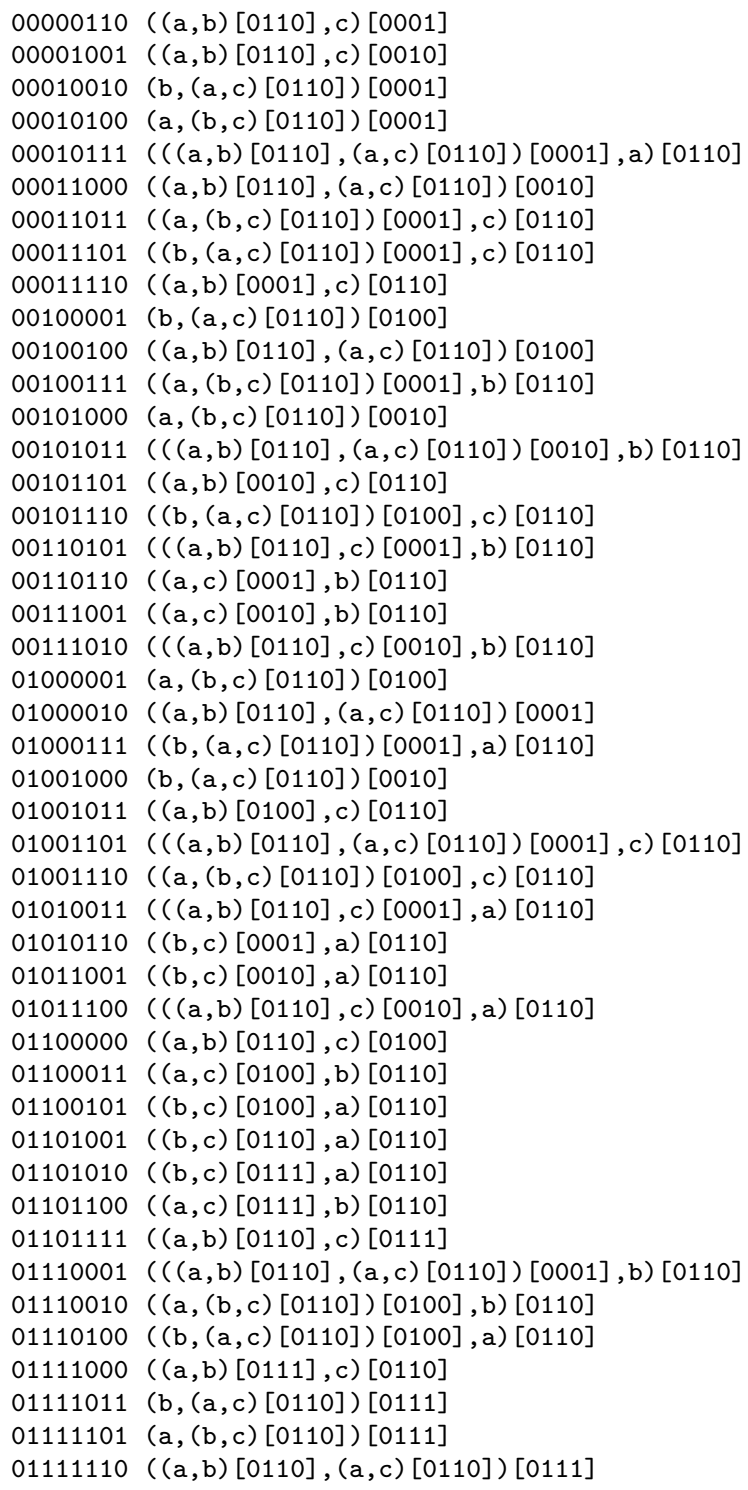

Figure 3.2: Replacement of XOR-Trivial 3-input Gates (not already listed in Fig. 3.1)

Before summarizing our main theorem for XOR-trivial gates we introduce the fol- 
lowing notation:

Notation 3 (Even and Odd Gates). An even gate is a gate whose truth table T has even parity, i.e., the Hamming weight $H^{\mathbb{N}}(T)$ is even. Otherwise the gate is called odd.

Example 4. The XOR gate with gate table [0110] and the 3-input gate with gate table [00010111] are even, whereas the OR gate with gate table [0111] is odd.

With this notation we obtain the following theorem for XOR-triviality:

Theorem 3 (XOR-trivial gates). For $d \in\{2,3\}$, the XOR-trivial d-input gates are exactly the even gates. Each of them can be replaced by at most one $(d-1)$-input non-XOR gate and at most 3 XOR gates.

Proof. The proof of Theorem 3 follows from the observations on XOR-trivial 2- and 3 -input gates given above and the fact that all 3-input gates listed in Fig. 3.1 and Fig. 3.2 are even.

Further observations on XOR-trivial gates. We observe that for the considered cases $d \in\{0,1,2,3\}$ the fraction of XOR-trivial $d$-input gates is at least $50 \%$, i.e., at least half of all such $d$-input gates can be replaced by cheaper ones.

As all practical circuit constructions described in $\$ 3.3$ are composed of $d \leq 3$-input gates and most of the 3 -input gates in these constructions are XOR-trivial, we do not investigate XOR-triviality for $d>3$ but leave this as an open problem.

\subsection{Efficient Circuit Constructions}

In this section we present several frequently used circuit building blocks which benefit from the circuit optimizations of $\$ 3.2$ as all occurring 3-input gates are XOR-trivial (= even) and hence can be replaced by at most one 2 -input non-XOR gate. The resulting sizes of the circuit constructions described in the following are summarized in Table 3.2 .

Publication Info: Parts of the following results are published in [KSS09, Sect. 3] and $\left[\mathrm{HKS}^{+} 10\right.$, Sect. 5.1.1] (3.3.2).

\subsubsection{Addition and Subtraction}

\subsubsection{Addition (ADD)}

An addition circuit (ADD) for adding two unsigned $\ell$-bit integers $\boldsymbol{x}, \boldsymbol{y}$ can be efficiently composed from a chain of 1-bit adders $(+)$, often called full-adders, as shown in Fig. 3.3 . 
Table 3.2: Size: Efficient Circuit Constructions (for $n$ unsigned $\ell$-bit values)

\begin{tabular}{|ll||c|c||c|}
\hline \multicolumn{1}{ll||}{ Circuit } & \multicolumn{2}{c||}{ Standard } & Free XOR \\
\hline \#gates & & 2-input & 3-input & 2-input non-XOR \\
\hline \hline ADD, SUB & $(3.3 .1)$ & 2 & $2 \ell-2$ & $\ell$ \\
ADDSUB & $(3.3 .1 .3$ & $\ell+1$ & $2 \ell$ & $\ell$ \\
\hline MUL (Textbook) & $(3.3 .2 .1$ & $\ell^{2}+2 \ell-2$ & $2 \ell^{2}-4 \ell+2$ & $2 \ell^{2}-\ell$ \\
MUL (Karatsuba) & $(3.3 .2 .2$ & & & $\approx 9 \ell^{1.6}-13 \ell-34 \ell$ \\
\hline CMP & $(3.3 .3 .1$ & 1 & $\ell-1$ & $\ell$ \\
MUX & $(3.3 .3 .2$ & 0 & $\ell$ & $\ell$ \\
MIN, MAX & $(3.3 .3 .3$ & $n-1$ & $2 \ell(n-1)+2$ & $2 \ell(n-1)+n+1$ \\
\hline
\end{tabular}

The first 1-bit adder has constant input $c_{1}=0$ and can be replaced by a smaller halfadder with two inputs. Each 1-bit adder has as inputs the carry-in bit $c_{i}$ from the previous 1-bit adder and the two input bits $x_{i}, y_{i}$. The outputs are the carry-out bit $c_{i+1}=\left(x_{i} \wedge y_{i}\right) \vee\left(x_{i} \wedge c_{i}\right) \vee\left(y_{i} \wedge c_{i}\right)=\left(x_{i}, y_{i}, c_{i}\right)$ [00010111] and the sum bit $s_{i}=x_{i} \oplus y_{i} \oplus c_{i}=\left(x_{i}, y_{i}, c_{i}\right)[01101001]$. All occurring gates are even and can be optimized to a small number of XOR gates. An equivalent construction for computing $c_{i+1}$ with the same number of non-XOR gates was given in [BPP00, BDP00].

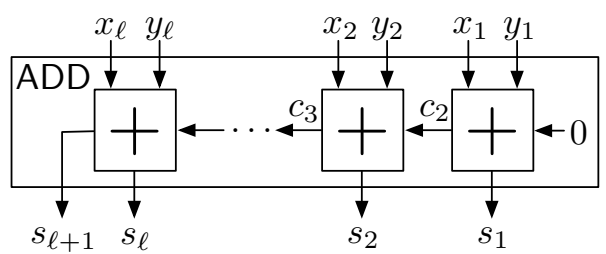

Figure 3.3: Circuit: Addition (ADD)

\subsubsection{Subtraction (SUB)}

Subtraction in two's complement representation is defined as $\boldsymbol{x}-\boldsymbol{y}=\boldsymbol{x}+\neg \boldsymbol{y}+1$. Hence, a subtraction circuit (SUB) can be constructed analogously to the addition circuit from 1-bit subtractors (-) as shown in Fig. 3.4. Each 1-bit subtractor computes the carryout bit $\left.c_{i+1}=\left(x_{i} \wedge \neg y_{i}\right) \vee\left(x_{i} \wedge c_{i}\right) \vee\left(\neg y_{i} \wedge c_{i}\right)=\left(x_{i}, y_{i}, c_{i}\right)\right)[01001101]$ and the difference bit $d_{i}=x_{i} \oplus \neg y_{i} \oplus c_{i}=\left(x_{i}, y_{i}, c_{i}\right)[10010110]$. The size of SUB is equal to that of ADD.

\subsubsection{Controlled Addition/Subtraction (ADDSUB)}

A controlled addition/subtraction circuit (ADDSUB) which can add or subtract two unsigned $\ell$-bit values $\boldsymbol{x}$ and $\boldsymbol{y}$ depending on a control input bit ctrl can be naturally constructed as combination of ADD, SUB, and controlled inversion (CNOT) which is 


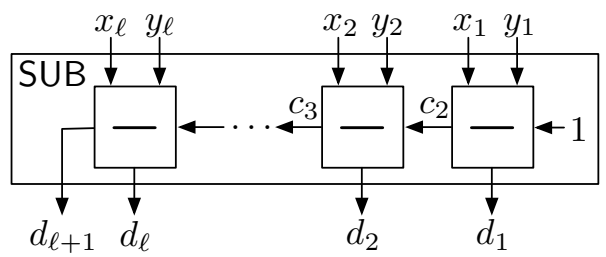

Figure 3.4: Circuit: Subtraction (SUB)

equal to (XOR): If $\operatorname{ctrl}=1, \boldsymbol{y}$ is subtracted from $\boldsymbol{x}$ by negating $\boldsymbol{y}$ and adding this to $\boldsymbol{x}+1$. Otherwise, $\boldsymbol{x}+\boldsymbol{y}+0$ is output. The resulting circuit is shown in Fig. 3.5 .

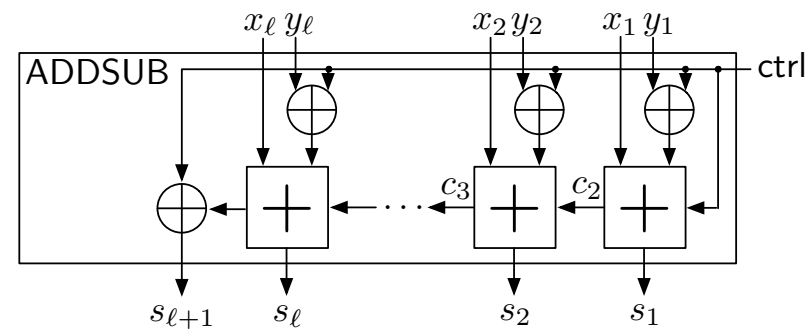

Figure 3.5: Circuit: Controlled Addition or Subtraction (ADDSUB)

Conversion. The ADDSUB circuit allows to convert back and forth between sign $/ \mathrm{mag}$ nitude and 2's complement representation as follows:

- sign/magnitude to 2 's complement: A signed integer value $\boldsymbol{x}$ given in sign/magnitude representation with $\ell$-bit magnitude can be converted into 2's complement by adding or subtracting the magnitude from 0 depending on the sign of $\boldsymbol{x}$.

- 2 's complement to sign/magnitude: An $(\ell+1)$-bit signed integer value $\boldsymbol{x}$ given in 2 's complement can be converted into sign/magnitude representation by adding or subtracting the least significant $\ell$ bits of $\boldsymbol{x}$ from 0 depending on the sign of $\boldsymbol{x}$, i.e., the most significant bit of $\boldsymbol{x}$.

\subsubsection{Multiplication}

We give two circuit constructions for multiplying two $\ell$-bit unsigned integer values based on textbook multiplication with size $\mathcal{O}\left(\ell^{2}\right)$ and based on Karatsuba-Ofman multiplication $\mathrm{KO} 62$ of size $\approx \mathcal{O}\left(\ell^{1.6}\right)$.

\subsubsection{Textbook Multiplication}

The "textbook method" for multiplying two $\ell$-bit unsigned integers $\boldsymbol{x}$ and $\boldsymbol{y}$ multiplies $\boldsymbol{x}$ with each bit of $\boldsymbol{y}$ and adds up all the properly shifted results according to the 
formula $\boldsymbol{x} \cdot \boldsymbol{y}=\sum_{i=1}^{\ell} 2^{i-1} y_{i} \cdot \boldsymbol{x}$. This results in a multiplication circuit (MUL) with $\ell^{2}$ of 1-bit multipliers (2-input AND gates) and $(\ell-1)$ of $\ell$-bit adders ADD.

\subsubsection{Fast Multiplication}

As observed by Karatsuba [KO62], multiplication of two large $\ell$-bit unsigned integers can be performed more efficiently using the following recursive method (details in Algorithm 2): $\boldsymbol{x}$ and $\boldsymbol{y}$ are split into two halves as $\boldsymbol{x}=\boldsymbol{x}_{h} 2^{\lceil\ell / 2\rceil}+\boldsymbol{x}_{\boldsymbol{l}}$ and $\boldsymbol{y}=$ $\boldsymbol{y}_{\boldsymbol{h}} 2^{\lceil\ell / 2\rceil}+\boldsymbol{y}_{\boldsymbol{l}}$. Then, the product can be computed as $\boldsymbol{x} \cdot \boldsymbol{y}=\left(\boldsymbol{x}_{\boldsymbol{h}} 2^{\lceil\ell / 2\rceil}+\boldsymbol{x}_{\boldsymbol{l}}\right)\left(\boldsymbol{y}_{\boldsymbol{h}} 2^{\lceil\ell / 2\rceil}+\right.$ $\left.\boldsymbol{y}_{\boldsymbol{l}}\right)=\boldsymbol{z}_{\boldsymbol{h}} 2^{2\lceil\ell / 2\rceil}+\boldsymbol{z}_{\boldsymbol{d}} 2^{\lceil\ell / 2\rceil}+\boldsymbol{z}_{\boldsymbol{l}}$. After computing $\boldsymbol{z}_{\boldsymbol{h}}=\boldsymbol{x}_{\boldsymbol{h}} \boldsymbol{y}_{\boldsymbol{h}}$ and $\boldsymbol{z}_{\boldsymbol{l}}=\boldsymbol{x}_{\boldsymbol{l}} \boldsymbol{y}_{\boldsymbol{l}}, \boldsymbol{z}_{\boldsymbol{d}}$ can be computed with only one multiplication as $\boldsymbol{z}_{\boldsymbol{d}}=\left(\boldsymbol{x}_{\boldsymbol{h}}+\boldsymbol{x}_{\boldsymbol{l}}\right)\left(\boldsymbol{y}_{\boldsymbol{h}}+\boldsymbol{y}_{\boldsymbol{l}}\right)-\boldsymbol{z}_{\boldsymbol{h}}-\boldsymbol{z}_{\boldsymbol{l}}$. This process is continued recursively until the numbers are smaller than some threshold $\theta$ $(\theta=19$ in case of free XOR as described below) and multiplied with the classical textbook method.

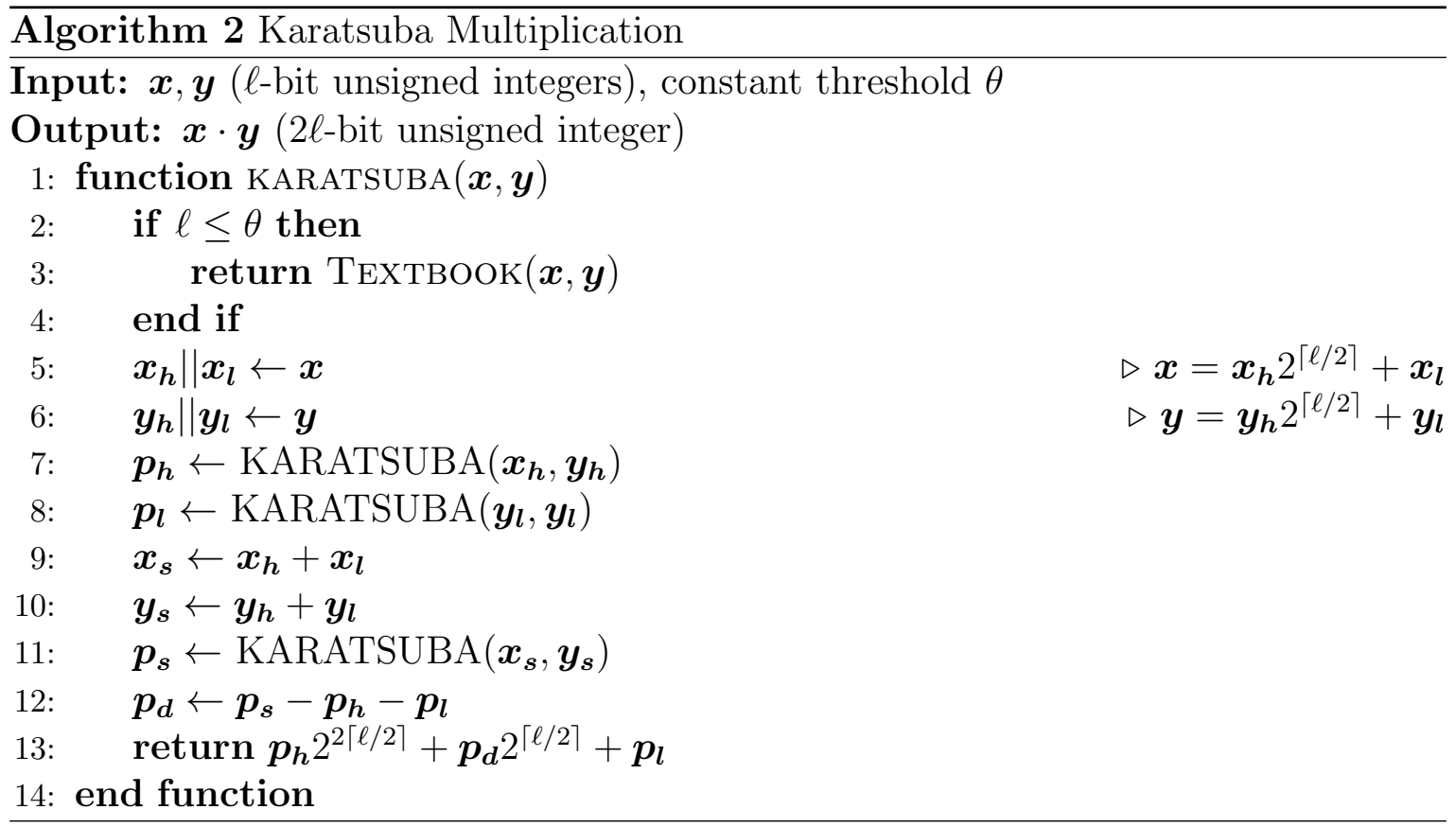

Overall, multiplying two $\ell$ bit numbers with Karatsuba's method requires three multiplications of $\ell / 2$ bit numbers and some additions and subtractions with linear bit complexity resulting in costs

$$
T_{\text {Kara }}(\ell)= \begin{cases}\mathcal{O}\left(\ell^{2}\right) & \text { if } \ell \leq \theta \\ 3 T_{\text {Kara }}(\ell / 2)+c \ell+d & \text { else }\end{cases}
$$

for constants $c$ and $d$. The master theorem [CLRS01, $\S 4.3 \mathrm{f}]$ yields asymptotic complexity $T_{\text {Kara }}(\ell) \in \mathcal{O}\left(\ell^{\log _{2} 3}\right) \approx \mathcal{O}\left(\ell^{1.585}\right)$. 


\subsubsection{Multiplication Circuit Complexity}

In TASTY (cf. \$5.2) we have implemented both methods for multiplication using the efficient addition and subtraction circuits of 3.3 .1 optimized for a small number of non-XOR gates. When XOR gates are "for free", we experimentally determined the optimal threshold to be $\theta=19$, i.e., Karatsuba multiplication is more efficient than textbook multiplication already for multiplication of 20 bit operands (cf. Fig. 3.6 and Table 3.3.

By interpolating through the points for bit length $\ell \in\{32,64,128\}$ and solving the resulting system of linear equations we obtain as approximation for the number of non-XOR gates

$$
T_{\text {Kara }}(\ell) \approx \begin{cases}2 \ell^{2}-\ell & \text { for } \ell \leq 19 \\ 9.0165 \ell^{1.585}-13.375 \ell-34 & \text { else. }\end{cases}
$$

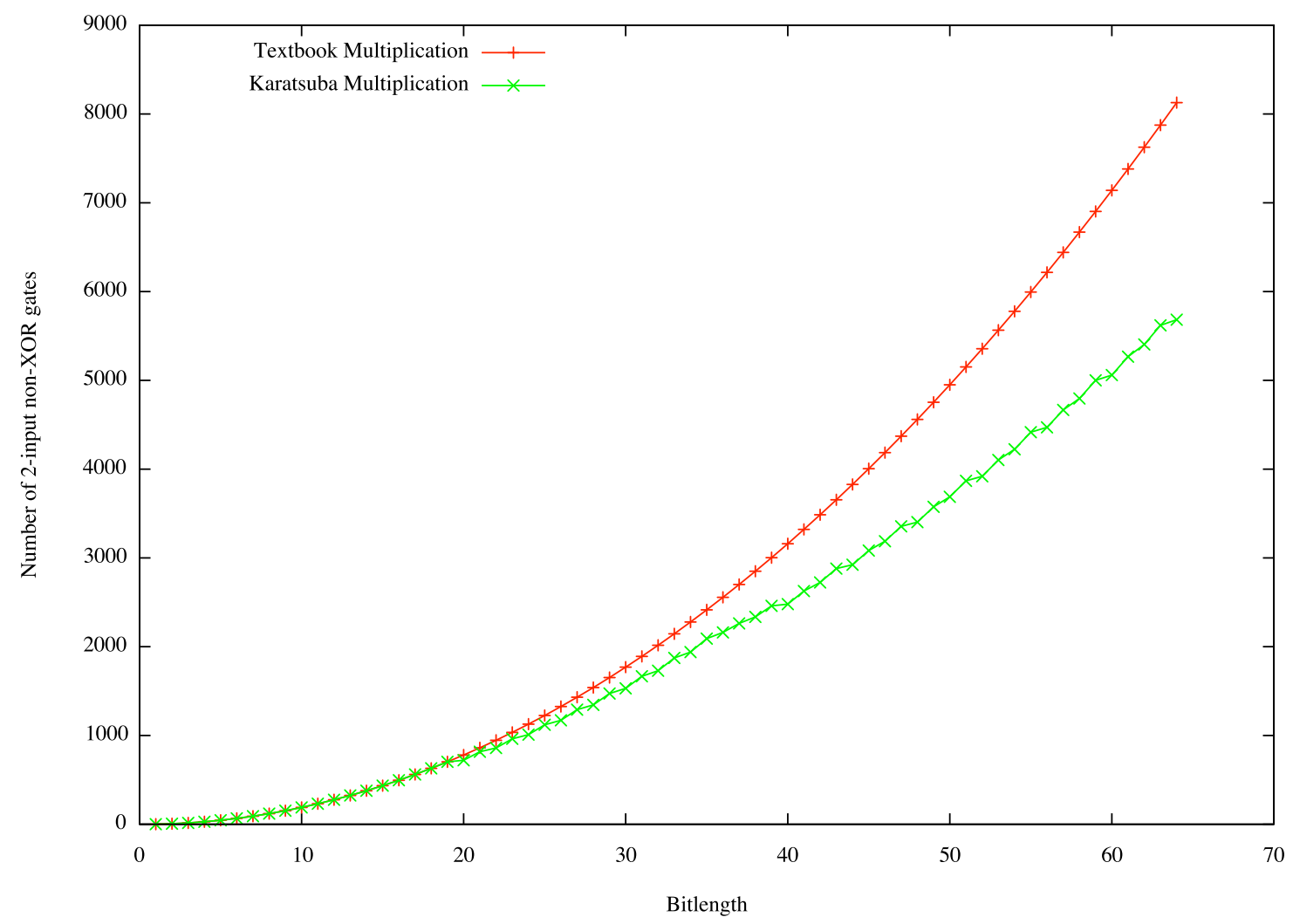

Figure 3.6: Size: Multiplication Circuits (with free XOR) 
Table 3.3: Size: Multiplication Circuits (in \# 2-input non-XOR gates)

\begin{tabular}{l|ccccc}
\hline Bitlength $\ell$ & 19 & 20 & 32 & 64 & 128 \\
\hline Textbook & 703 & 780 & 2,016 & 8,128 & 32,640 \\
Karatsuba & 703 & 721 & 1,729 & 5,683 & 17,973 \\
\hline Improvement & $0.0 \%$ & $7.6 \%$ & $14.2 \%$ & $30.1 \%$ & $44.9 \%$ \\
\hline
\end{tabular}

\subsubsection{Comparison, Minima and Maxima}

\subsubsection{Comparison}

Comparing two $\ell$-bit unsigned integers $\boldsymbol{x}$ and $\boldsymbol{y}$ is equivalent to computing the function

$$
z=[\boldsymbol{x}>\boldsymbol{y}]:= \begin{cases}1 & \text { if } \boldsymbol{x}>\boldsymbol{y} \\ 0 & \text { else }\end{cases}
$$

As shown in Fig. 3.7, a comparison circuit (CMP) can be composed from $\ell$ sequential 1-bit comparators $(>)$. The first 1-bit comparator has constant input $c_{1}=0$ and can be replaced by a 2-input gate. Each 1-bit comparator has as inputs the carry-in bit $c_{i}$ from the previous 1-bit comparator and the two input bits $x_{i}, y_{i}$. Its output is the carry-out bit $c_{i+1}=\left(x_{i} \wedge y_{i}\right) \vee\left(x_{i} \wedge c_{i}\right) \vee\left(y_{i} \wedge c_{i}\right)=\left(x_{i}, y_{i}, c_{i}\right)[01001101]$.

Note, this improved bit comparator computes the same function as the carry output of the subtraction circuit of $\S 3.3 .1 .2$ as $[\boldsymbol{x}>\boldsymbol{y}] \Leftrightarrow[\boldsymbol{x}-\boldsymbol{y}-1 \geq 0]$, this coincides with an underflow in the corresponding subtraction denoted by subtractor's most significant output bit $d_{\ell+1}$.

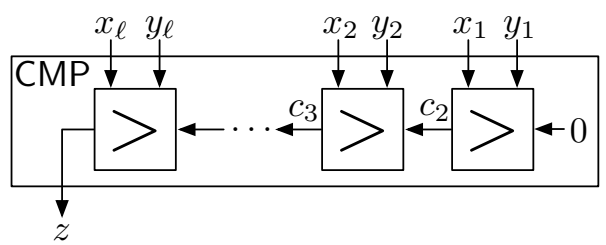

Figure 3.7: Circuit: Comparison (CMP)

Modifications. Comparison circuits for $[\boldsymbol{x}<\boldsymbol{y}],[\boldsymbol{x} \geq \boldsymbol{y}]$, or $[\boldsymbol{x} \leq \boldsymbol{y}]$ can be obtained from the comparison circuit for $[\boldsymbol{x}>\boldsymbol{y}]$ by interchanging $\boldsymbol{x}$ with $\boldsymbol{y}$ and/or setting the initial carry to $c_{1}=1$.

A circuit to check if two values are equal $([\boldsymbol{x}=\boldsymbol{y}])$, can be constructed analogously by computing $c_{i+1}=\left(x_{i}=y_{i}\right) \wedge c_{i}=\left(x_{i}, y_{i}, c_{i}\right)$ [00001001] with $c_{1}=1$. Similarly, a circuit for checking inequality $([\boldsymbol{x} \neq \boldsymbol{y}])$ computes $c_{i+1}=\left(x_{i} \neq y_{i}\right) \vee c_{i}=\left(x_{i}, y_{i}, c_{i}\right)[01101111]$ 
with $c_{1}=0$. These circuits for equality and inequality have the same size as the circuit constructions given in $\mathrm{KS} 08 \mathrm{a}$.

\subsubsection{Multiplexer}

An $\ell$-bit multiplexer circuit MUX selects its output $\boldsymbol{z}$ to be its left $\ell$-bit input $\boldsymbol{x}$ if the input selection bit $c$ is 0 , respectively its right $\ell$-bit input $\boldsymbol{y}$ otherwise. As shown in Fig. 3.8, this circuit can be composed from $\ell$ parallel 1-bit multiplexers $(Y)$, where $\mathrm{Y}$ computes $z_{i}=\left(x_{i}, y_{i}, c\right)[01010011]$. After optimization, this circuit has the same size as the circuit construction given in [KS08a].

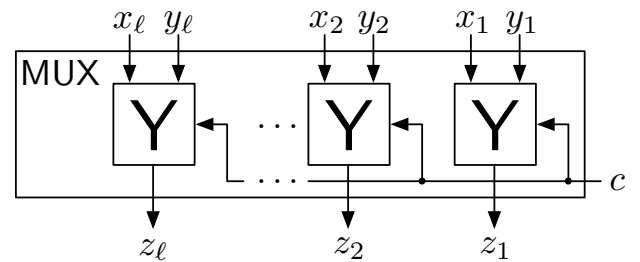

Figure 3.8: Circuit: Multiplexer (MUX)

\subsubsection{Minimum/Maximum Value and Index}

We show how to combine the blocks for comparison and multiplexing into a circuit MIN which selects the minimum value $\boldsymbol{m}$ and minimum index $\boldsymbol{i}$ of a list of $n$ unsigned $\ell$-bit integers $\boldsymbol{x}_{0}, \ldots, \boldsymbol{x}_{n-1}$, i.e., $\forall j \in\{0, \ldots, n-1\}:\left(\boldsymbol{m}<\boldsymbol{x}_{\boldsymbol{j}}\right) \vee\left(\boldsymbol{m}=\boldsymbol{x}_{j} \wedge \boldsymbol{i} \leq j\right)$.

Example 5. For the list 3,2,5,2 the output of MIN would be $\boldsymbol{m}=2$ and $\boldsymbol{i}=1$ as the leftmost minimum value of 2 is at position 1.

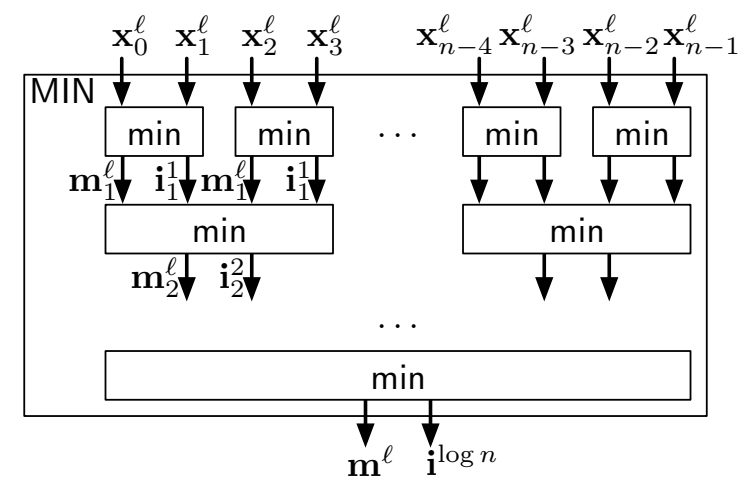

Figure 3.9: Circuit: Minimum (MIN)

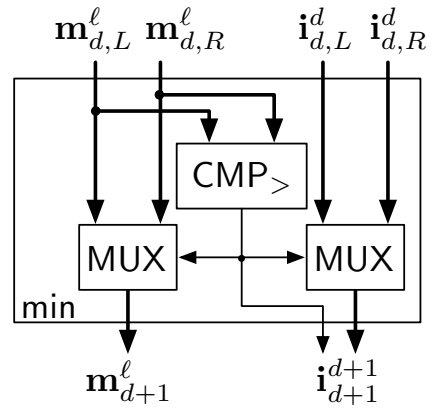

Figure 3.10: Block: Minimum (min)

To minimize circuit size we arrange a tournament-style circuit so that some of the index wires can be reused and eliminated. That is, at depth $d$ of the resulting tree we 
keep track of the $\ell$-bit minimum value $\boldsymbol{m}^{2}$ of the sub-tree containing $2^{d}$ values but store and propagate only the $d$ least significant bits $\boldsymbol{i}_{d}^{d}$ of the minimum index.

W.l.o.g. we assume that $n$ is a power of two in the following and hence the minimum index can be represented with exactly $\log n$ bits. (If $n$ is not a power of two, the circuit given in the following can be constructed for the next power of two and optimized afterwards.) More specifically, the minimum value and minimum index are selected pairwise in a tournament-like way using a tree of minimum blocks ( $\mathrm{min}$ ) as shown in Fig. 3.9. As shown in Fig. 3.10, each minimum block at depth $d$ gets as inputs the minimum $\ell$-bit values $\boldsymbol{m}_{d, L}^{\ell}$ and $\boldsymbol{m}_{d, R}^{\ell}$ of its left and right subtrees $T_{L}, T_{R}$ and the $d$ least significant bits of their minimum indices $\boldsymbol{i}_{d, L}^{d}$ and $\boldsymbol{i}_{d, R}^{d}$, and outputs the minimum $\ell$-bit value $\boldsymbol{m}_{d+1}^{\ell}$ and $(d+1)$-bit minimum index $\boldsymbol{i}_{d+1}^{d+1}$ of the tree. First, the two minimum values are compared with a comparison circuit (cf. \$3.3.3.1). If the minimum value of $T_{L}$ is bigger than that of $T_{R}$ (in this case, the comparison circuit outputs value 1 ), $\boldsymbol{m}_{d+1}^{\ell}$ is chosen to be the value of $T_{R}$ with an $\ell$-bit multiplexer block (cf. \$3.3.3.2). In this case, the minimum index $\boldsymbol{i}_{d+1}^{d+1}$ is set to 1 concatenated with the minimum index of $T_{R}$ using another $d$-bit multiplexer. Alternatively, if the comparison yields 0 , the minimum value of $T_{L}$ and the value 0 concatenated with the minimum index of $T_{L}$ are output.

Overall, the size of the efficient minimum circuit is $\left|\mathrm{MIN}^{\ell, n}\right|=(n-1) \cdot\left(\left|\mathrm{CMP}^{\ell}\right|+\right.$ $\left.\left|\mathrm{MUX}^{\ell}\right|\right)+\sum_{j=1}^{\log n} \frac{n}{2^{j}}\left|\mathrm{MUX}^{j-1}\right|$. When XOR gates are free, the resulting number of non$\mathrm{XOR}$ gates is $(n-1) \cdot(\ell+\ell)+n \sum_{j=1}^{\log n} \frac{j-1}{2^{j}}<2 \ell(n-1)+n\left(1+\frac{1}{n}\right)=2 \ell(n-1)+n+1$.

Modifications. A circuit MAX which computes the maximum value and index can be constructed similarly by constructing max blocks which use $\mathrm{CMP}_{<}$instead of $\mathrm{CMP}_{>}$. When only the minimum (maximum) value or only the minimum (maximum) index is needed, the corresponding multiplexer in the last min $(\max )$ block can be omitted.

\subsection{Applications: Secure Comparison and Auctions}

The efficient circuit building blocks described in $\$ 3.3$ with optimizations for free XORs can serve as basis for highly efficient protocols for secure comparison (3.4.1) and auction protocols $(\$ 3.4 .2$ as described next.

Publication Info: Parts of the following results are published in [KSS09, Sect. 5].

\subsubsection{Comparison (Millionaires Problem)}

The "Millionaires problem" was introduced by Yao in [Yao82] as motivation for secure computation: two millionaires want to securely compare their respective private input

\footnotetext{
${ }^{2}$ To simplify presentation we write the number of bits of a variable as superscript index.
} 
values (e.g., their amount of money) without revealing more information than the outcome of the comparison to the other party. More concretely, client $\mathcal{C}$ holds a private $\ell$-bit value $x$ and server $\mathcal{S}$ holds a private $\ell$-bit value $y$. The output bit $z=[x<y]$ should be revealed to both. In the semi-honest case it is sufficient to consider the case where $\mathcal{C}$ obtains the output and forwards it to $\mathcal{S}$.

An example application that can be reduced to multiple invocations of secure comparison is the secure computation of the $k^{\text {th }}$-ranked element of the union of two datasets held by two parties AMP04.

When instantiated efficiently, Yao's Garbled Circuit (GC) protocol (cf. \$2.3.1.1) is the most efficient solution to solve the Millionaires problem for computationally bounded players. Many other protocols for secure comparison based on Homomorphic Encryption (HE) were proposed which work in a stronger model, where one player is computationally unbounded [Fis01, BK04], or extended the Millionaires problem to other scenarios such as online auctions [DGK07, DGK08b, DGK08a] (cf. §3.4.2.2), or multiparty computation [GSV07].

In the following, we show that evaluating our improved comparison circuit of \$3.3.3.1, using an efficient GC-based Secure Function Evaluation (SFE) protocol with free $\mathrm{XORs}$, is more efficient than previous comparison protocols.

\subsubsection{Comparison with Pre-Computations}

In many practical application scenarios it is beneficial to shift as much of the computation and communication cost of a protocol into an interactive setup (pre-computation) phase, which is executed before the parties' inputs are known, while the parties' workload is low. In contrast to many protocols based on $\mathrm{HE}$, almost the entire cost of GC-based protocols can be shifted into the setup phase.

As proposed by Yao Yao86, an efficient solution for the Millionaires problem is obtained by evaluating a comparison circuit with an SFE protocol (cf. \$2.3.1), where $\mathcal{C}$ provides her $\ell$-bit input $x, \mathcal{S}$ provides his $\ell$-bit input $y$, and $\mathcal{C}$ obtains the output bit $z=[x<y]$. We instantiate this protocol with the following efficient primitives in the Random Oracle (RO) model: As comparison circuit we use our optimized comparison circuit of $\$ 3.3 .3 .1$ with $\ell$ 2-input non-XOR gates. As efficient GC protocol we use garbled row reduction [NPS99] with free XORs [KS08a] (cf. 2.2.2.3). Hence, the resulting GC has size $\ell \cdot 3(t+1)$ bits. For Oblivious Transfer (OT) we use the protocol of [NP01] with pre-computations of [Bea95] (cf. \$2.2.3).

\subsubsection{Complexity Evaluation}

In the following we show that the GC-based comparison protocol has a smaller complexity than previous HE-based protocols. 
Computation Complexity. As our improved circuit for integer comparison described in 3.3.3.1 consists of $\ell$ non-XOR 2-input gates, $\mathcal{C}$ needs to invoke the underlying cryptographic hash-function (e.g., SHA -256 for $t=128$ bit symmetric security) exactly $\ell$ times to evaluate the GC (cf. \$2.2.2.4). All other operations are negligible (XOR of bitstrings). Hence, the computational complexity of the online phase of our protocol is negligible compared to that of protocols based on HE. Even with an additional setup phase, those HE-based protocols need to invoke a few modular operations in the online phase for each input bit which are usually by several orders of magnitude more expensive than the evaluation of a cryptographic hash function used in our protocols. For large $\ell$ or parallel comparisons, our protocols have smaller overall computation complexity than comparison protocols based on $\mathrm{HE}$, as the number of public-key operations is independent of the bitlength $\ell$ when using the efficient OT extension of [IKNP03] as described in $\$ 2.2 .3$.

Communication Complexity. In Table 3.4 we compare the communication complexity of our GC-based comparison protocol with that of previous HE-based protocols as described in the following. The names and sizes of the security parameters are chosen according to $\$ 2.1 .1 .2$.

In our protocol, the communication complexity of the setup phase consists of $\ell \cdot 3(t+$ 1) bits for sending the GC, $6 \ell t$ bits for pre-computing OTs (using the OT protocol of [NP01] over Elliptic Curves (ECs), and $\ell$ bits for allowing $\mathcal{C}$ to decrypt the output, i.e., asymptotically $9 \ell t$ bits. The communication complexity of the online phase consists of $\ell(t+1)$ bits for sending $\mathcal{S}$ 's garbled inputs $\widetilde{y}$, and $\sim 2 \ell t$ bits for the online OT phase for $\mathcal{C}$ 's garbled inputs $\widetilde{y}$, i.e., asymptotically $3 \ell t$ bits. When pre-computations are not possible, the two phases can be combined and OT needs not to be pre-computed.

The comparison protocol of Fischlin [Fis01] uses the Goldwasser-Micali XOR HE scheme [GM84] and has asymptotic communication complexity $\ell T(\kappa+1)$, where $\kappa$ is the statistical correctness parameter (e.g., $\kappa=40$ ). The comparison protocol of Blake and Kolesnikov [BK04] requires to transfer $2 \ell$ ciphertexts of an additively HE scheme which can be instantiated with any of the schemes presented in \$2.2.1.1: Paillier [Pai99] (as proposed in [BK04]), Damgård-Geisler-Krøigaard [DGK07, DGK08b, DGK08a] (as used for example in [EFG $\left.{ }^{+} 09\right]$ ), or lifted EC-ElGamal [Gam85] (as used for example in $\left.\left[\mathrm{BBC}^{+} 10\right]\right)$.

Comparison with pre-computations. As shown in Table 3.4, the communication complexity of the online phase of our improved GC-based comparison protocol is substantially lower than that of previous HE-based protocols. The GC-based protocol improves over the online communication complexity of the best HE-based protocol $\left(\right.$ BK04] + EC-ElGamal Gam85]) by a factor of $\frac{8 \ell t}{3 \ell t} \approx 2.7$ times. 
Table 3.4: Communication Complexity: Comparison Protocols (on $\ell$-bit values)

\begin{tabular}{|c|c|c|c|c|c|}
\hline \multirow{2}{*}{ Protocol } & \multirow{2}{*}{$\begin{array}{l}\text { Asymptotic } \\
\text { Complexity }\end{array}$} & \multicolumn{4}{|c|}{ 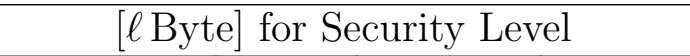 } \\
\hline & & ultra-short & short & medium & long \\
\hline [Fis01] & $\overline{(k+1) \ell T}$ & 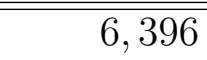 & 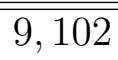 & $\overline{12,464}$ & $\overline{16,646}$ \\
\hline [BK04] + Pai99 & $4 \ell T$ & 624 & 888 & 1,216 & 1,624 \\
\hline BK04] DGK07 & $2 \ell T$ & 312 & 444 & 608 & 812 \\
\hline$[$ BK04] EC-Gam85 & $8 \ell t$ & 80 & 96 & 112 & 128 \\
\hline \multicolumn{6}{|l|}{ GC-based comparison } \\
\hline Setup Phase & $9 \ell t$ & 90 & 108 & 126 & 144 \\
\hline Online Phase & $3 \ell t$ & 30 & 36 & 42 & 48 \\
\hline Combined & $10 \ell t$ & 100 & 120 & 140 & 160 \\
\hline
\end{tabular}

Comparison in parallel and without pre-computations. We note that when many comparisons are performed in parallel, the communication complexity of our protocol is improved by $2 \ell t$ as OTs can be extended efficiently (cf. s2.2.3.4): the setup phase has asymptotic communication complexity $7 \ell t$; the combined protocol without pre-computations has $8 \ell t$ communication. In this case, even if the application scenario does not allow pre-computations, the communication complexity of our GCbased comparison protocol is similar to that of the best HE-based comparison protocol of [BK04] instantiated with EC-ElGamal Gam85].

\subsubsection{Auctions}

In standard auction systems such as Ebay, the auctioneer learns the inputs of all bidders and hence can deduce valuable information about the bidding behavior of unsuccessful bidders or cheat while computing the auction function depending on bidders' input values. To overcome this, a secure protocol can be used instead. Bidders provide their bids in "encrypted" form to the protocol which allows the auctioneer to compute the auction function without learning the bids.

Next, we show how our efficient circuit constructions can be used to improve two previously proposed secure auction systems: in offline auctions all bids are collected before the auction function is computed $(\$ 3.4 .2 .1)$, whereas in online auctions the bids are input dynamically and the current highest bid is published (\$3.4.2.2).

\subsubsection{Offline Auctions}

The offline auction system of [NPS99] evaluates the auction function in the Secure Multi-Party Computation (SMPC) setting with two servers as described in $\$ 2.3 .2 .1$; The auction issuer creates the $\mathrm{GC}$ and the auctioneer evaluates it on the garbled bids provided by the bidders. Finally, the auctioneer publishes the outcome of the auction 
which can be verified with the help of the auction issuer.

In order to run a first-price auction which outputs the maximum bid and the index of the maximum bidder, a circuit for the maximum value and its index (cf. \$3.3.3.3) needs to be evaluated. The GC for first-price auctions with $n$ bidders and $\ell$-bit bids used in [NPS99] requires approximately $15 n \ell$ table entries when using the GC method of [NPS99] without free XOR (cf. \$2.2.2.3). Instead, our improvements result in a $\mathrm{GC}$ with approximately $3 \cdot 2 \ell n=6 \ell n$ table entries, when evaluating our optimized maximum circuit presented in $\$ 3.3 .3 .3(\approx 2 \ell n$ non-XOR gates) with the GC method of [NPS99] and free XORs. The communication between the two computation servers for sending the GClas well as the auction issuer's time for creating the GClare improved by a factor of $\frac{15}{6}=2.5$. The time for evaluating the $\mathrm{GC}$ is reduced as well.

We note that also other types of auctions, such as second-price auctions, benefit from our improved circuit constructions as these auction functions are also composed from comparisons and multiplexers for which we give more efficient circuit constructions with free XORs.

\subsubsection{Online Auctions}

In an on-line auction system, each bidder submits a maximum bid to the system and goes offline afterwards. The system automatically bids for each bidder until their respectively specified maximum is exceeded. In contrast to offline auctions, new maximum bids can be submitted dynamically until the auction ends, e.g., at a specified time. Clearly, the maximum bid is confidential information as both the auctioneer and other bidders could exploit this information to their advantage.

The secure online auction system proposed in [DGK07, DGK08a, DGK08b] extends the idea of splitting the computation of the auction function between two servers, the auctioneer (called server) and another party (called assisting server) who are assumed not to collude. Each bidder secret-shares her bid and sends it to both servers over a secure channel. The two servers dynamically compare the secret-shared maximum bids with the public value of the current highest bid. The output of the comparison is public and determines whether the bidder is still in the game and wants to raise the bid, say by some fixed amount agreed in advance. The secure comparison guarantees that neither of the two servers learns any information on the maximum bid other than the comparison result. A detailed description of the scenario can be found in [DGK08b].

HE-based Online Auctions. The protocol proposed by Damgård, Geisler and Kroigård [DGK07, DGK08a, DGK08b] is a modification of a HE-based comparison protocol, where one input is bitwise secret-shared between the two parties. The communication complexity of this protocol is the same as for the HE-based comparison protocols of \$3.4.1. 
GC-based Online Auctions. We propose to use the efficient GC-based comparison protocol of $\$ 3.4 .1$ for the online auctions with inputs given in different forms: the maximum bid is secret-shared between both players (see below for a simple technique to use such inputs in GC) and the other input is publicly known to both parties (e.g., can be treated as a private input of circuit constructor $\mathcal{S}$ ). The resulting GC-based protocol for online auctions has exactly the same performance as our solution for the Millionaires problem described in $\$ 3.4 .1$.

Performance Comparison. As the complexity of the HE and GC-based online auction protocols is similar to the complexity of the corresponding comparison protocols of 33.4 .1 , the GC-based protocol outperforms the HE-based protocol for the same reasons. In particular, the possibility to move all expensive operations into the setup phase, which can be executed during idle times (whenever no new bids are received), is very beneficial for the online auctions scenario as this enables the bidders to instantly see if their current bid was successful or if another bidder meanwhile gave a higher bid. This feature is important especially towards the end of the auction, where the frequency of bids is usually very high.

Secret-Shared Inputs. As proposed in [FPRS04], a bit $b$ can be secret-shared between $\mathcal{C}$ holding share $b_{\mathcal{C}}$ and $\mathcal{S}$ holding share $b_{\mathcal{S}}$, with $b=b_{\mathcal{C}} \oplus b_{\mathcal{S}}$. A secret-shared input bit $b$ can be converted into a garbled input $\widetilde{b}$ for $\mathcal{C}$ using a 1-out-of-2 OT protocol (cf. $\$ 2.2 .3): \mathcal{C}$ inputs $b_{\mathcal{C}}$ and $\mathcal{S}$ inputs the two corresponding garbled values in the usual order $\vec{b}^{0}, \widetilde{b}^{1}$ if $b_{\mathcal{S}}=0$ or swaps them to $\widetilde{b}^{1} \widetilde{b}^{0}$ otherwise. It is easy to verify that $\mathcal{C}$ obliviously obtains the correct garbled value $\widetilde{b}$ for the shared bit $b$. 


\section{Chapter 4}

\section{Hardware-Assisted Garbled Circuit Protocols}

In this chapter we describe how protocols based on Garbled Circuits (GCs) can be enhanced by using hardware (HW). We show how a tamper-proof [HW token can generate GCs on-the-fly to remove the necessity of generating and transferring GCs over the network $(\$ 4.1)$. Afterwards, in $\$ 4.2$, we show how GCs can be evaluated efficiently in $\mathrm{HW}$, and extend and evaluate the practicality of One-Time Programs (OTPs) for protecting against arbitrary side-channel attacks. As application we show in 4.3 how a $\mathrm{HW}$ token can be used for secure outsourcing of data and arbitrary computations thereon in a cloud computing scenario.

\subsection{Creating Garbled Circuits with Hardware Token}

The techniques described in this section allow to make the communication complexity of Garbled Circuit (GC)-based Secure Function Evaluation (SFE) protocols independent of the size of the evaluated functionality. Instead of generating and transferring the GC over the network, we propose to use a low-cost tamper-proof hardware (HW) token which is issued and trusted by one party and generates the GC on behalf of her. The token is cheap as it executes only symmetric-key operations (e.g., SHA and AES) and has small constant-size RAM (much smaller than the size of the circuit), but we do not resort to implementing expensive secure external RAM. We provide two solutions; in one, $\mathcal{T}$ keeps state in secure non-volatile storage (a monotonic counter), while in the other, $\mathcal{T}$ maintains no long-term state.

Publication Info: Parts of the following results are published in [JKSS10a and JKSS10b. 


\subsubsection{Motivation and Setting}

The communication complexity of GC-based protocols is dominated by the transfer of the $\mathrm{GC} \widetilde{C}$, as for each (non-XOR) gate, a garbled table has to be sent (cf. \$2.2.2.4), e.g., the GC for AES has size 0.5 MB [PSSW09]. Further, if security against more powerful adversaries is required, the use of the standard cut-and-choose technique implies transfer of multiple GCs (cf. \$2.3.1.2). While transmission of this large amount of data is possible for exceptional occurrences, in most cases, the network will not be able to sustain the resulting traffic. This holds especially for larger-scale deployment of secure computations, e.g., by banks or service providers, with a large number of customers. Additional obstacles include energy consumption required to transmit/receive the data, and the resulting reduced battery life in mobile clients, such as smartphones ${ }^{1}$

In this section we show how to remove this expensive communication requirement by generating the $\mathrm{GC}$ locally with a secure $\mathrm{HW}$ token $\mathcal{T}$. The token is issued by GC creator $\mathcal{S}$ and given to $\mathrm{GC}$ evaluator $\mathcal{C}$. $\mathcal{C}$ communicates locally with $\mathcal{T}$, and remotely with $\mathcal{S}$, to obtain the GC. There is no direct channel between $\mathcal{T}$ and $\mathcal{S}$, but of course $\mathcal{C}$ can pass (and potentially interfere with) messages between $\mathcal{T}$ and $\mathcal{S}$. $\mathcal{T}$ is created by $\mathcal{S}$, so $\mathcal{S}$ trusts $\mathcal{T}$; however, as $\mathcal{C}$ does not trust $\mathcal{S}$, she also does not trust the token $\mathcal{T}$ to behave honestly $\left.\right|^{2}$

Hardware assumption. We assume $\mathcal{T}$ is tamper-proof. We argue that this assumption is reasonable. Indeed, while every token can likely be broken into, given sufficient resources (see for example the physical breach of the Trusted Platform Module (TPM) Sec10]), we are motivated by the scenarios where the payoff of the break is far below the cost of the break. This holds for relatively low-value transactions such as cell phone or TV service, where the potential benefit of the attack (e.g., free TV for one user) is not worth the investment of thousands or tens of thousands of dollars to break into the card. For higher-value applications one could raise the cost of the attack by using a high-end token $\mathcal{T}$, e.g., a smart card certified at FIPS 140-2, level 3 or 4 .

Hardware restrictions. As we assume the token to be produced in large quantities, we try to minimize its costs (e.g., chip surface) and make the assumptions on it as weak as possible. In particular our token requires only restricted computational capabilities (no public-key operations) and a small, constant amount of secure RAM. We consider $\mathcal{T}$ with and without a small, constant amount of secure non-volatile storage.

\footnotetext{
${ }^{1}$ In some cases, the impact can be mitigated by creating and transferring GCs in the setup phase. However, this is not fully satisfactory. Firstly, even more data needs to be transferred since demand cannot be perfectly predicted. Further, this creates other problems, such as requiring large long-term storage on client devices.

${ }^{2}$ Note, if $\mathcal{C}$ in fact trusts $\mathcal{T}$ to behave honestly, then there exists a trivial solution, where $\mathcal{C}$ would let $\mathcal{T}$ compute the function on her inputs [IS05.
} 


\subsubsection{Related Work}

Related work on using tokens for secure computations can be divided into the following three categories, summarized in Table 4.1.

Table 4.1: Secure Protocols using Hardware Tokens. Columns denote the number of tokens $N$, who trusts the token(s), if token(s) are stateful or stateless, and perform public-key (PK) operations. Properties more desired for practical applications in bold font.

\begin{tabular}{|c|c|c|c|c|c|c|}
\hline & Reference & Functionality & $N$ & Trust & Stateful & PK \\
\hline \multirow[t]{4}{*}{ A) } & HMU05 & \begin{tabular}{|l|l|l} 
UC & commitment
\end{tabular} & 2 & both & yes & yes \\
\hline & Kat07, DNW09] & UC commitment & 2 & issuer & yes & yes \\
\hline & CGS08 & $\overline{\mathrm{UC}}$ commitment & 2 & issuer & no & yes \\
\hline & MS08 & UC commitment & 1 & issuer & yes & no \\
\hline \multirow[t]{6}{*}{ B) } & HL08 & Set Intersection, ODBS & 1 & both & yes & no \\
\hline & GT08 & Non-Interactive OT & 1 & both & yes & yes \\
\hline & [TV09] & Verif. Enc., Fair Exch. & 1 & both & yes & yes \\
\hline & Kol10 & $\mathrm{OT}$ & 1 & issuer & no & no \\
\hline & $\mathrm{FPS}^{+} 11$ & Set Intersection & 1 & issuer & yes & no \\
\hline & [DSV10] & Non-Interactive OT & 1 & issuer & yes & no \\
\hline \multirow[t]{3}{*}{ C) } & $\mathrm{FFP}^{+} 06$ & SFE & 2 & both & yes & yes \\
\hline & [IS06, Ili09, IS10] & SFE & 1 & both & yes & yes \\
\hline & This Work & $\overline{\mathrm{SFE}}$ & 1 & issuer & yes $/$ no & no \\
\hline
\end{tabular}

A) Setup assumptions for the Universal Composability (UC) framework. As shown in [CLOS02, UC-secure SFE protocols can be constructed from UC commitments. In turn, $\mathrm{UC}$ commitments can be constructed from signature cards trusted by both parties [HMU05], or from tamper-proof tokens created and trusted only by the issuing party [Kat07, MS08, CGS08, DNW09]. Here, CGS08] consider stateless tokens, and [MS08] require only one party to issue a token. This line of research mainly addresses the feasibility of UC-secure computation based on tamper-proof $\mathrm{HW}$ and relies on expensive primitives such as generic zero-knowledge proofs. Our token-based SFE protocols do not achieve UC-security, but are far more practical.

B) Efficiency Improvements for Specific Functionalities. Efficient protocols with a tamper-proof token trusted by both players have been proposed for specific functionalities such as set intersection and oblivious database search (ODBS) [HL08], non-interactive Oblivious Transfer (OT) GT08, and verifiable encryption and fair exchange [TV09]. Protocols secure against covert adversaries with one token trusted by 
its issuer only were proposed for interactive OT [Kol10] (stateless token) and set intersection [FPS ${ }^{+} 11$ ] (stateful token). Non-interactive OT with security against malicious adversaries with a stateful token was proposed recently in [DSV10]. In contrast, we solve the general SFE problem.

C) Efficiency Improvements for Arbitrary Functionalities. Clearly, SFE is efficient if aided by one or multiple Trusted Third Partys (TTPs), that simply compute(s) the function: SFE aided by multiple smartcards as TTPS was considered in $\left[\mathrm{FFP}^{+} 06\right]$; the Faerieplay project uses a cryptographic co-processor as single TTP [IS06, Ili09, IS10]. The protocol of [HS10] provides SFE with aborts secure against malicious adversaries where only one party obtains the output. Their construction uses a TTP that generates the GC and guarantees its well-formedness with a signature. By adapting our techniques of \$4.1.4, the TTP can be implemented with constant memory within a $\mathrm{HW}$ token. In contrast to these protocols we do not use any TTP - our token is only trusted by its issuer.

\subsubsection{Architecture, System and Trust Model}

We present in detail our setting, players, and $\mathrm{HW}$ and trust assumptions.

As shown in Fig. 4.1, we extend the two-party SFE setting consisting of client $\mathcal{C}$ and server $\mathcal{S}$ with a third party, the tamper-proof token $\mathcal{T}$, issued and trusted by $\mathcal{S}$ and given to $\mathcal{C}$. Our goal is to let $\mathcal{C}$ and $\mathcal{S}$ evaluate the public function $f$ on their respective private inputs $x$ and $y$ with the help of $\mathcal{T}$.

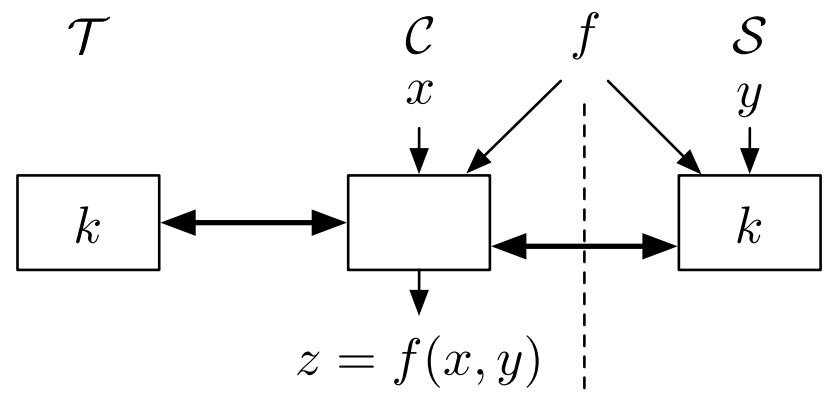

Figure 4.1: Model Overview: Token-assisted SFE

Communication. $\mathcal{C} \leftrightarrow \mathcal{S}$ : We view this as an expensive channel. Communication $\mathcal{C} \leftrightarrow \mathcal{S}$ flows over the Internet, and may include a wireless or cellular link. This implies small link bandwidth and power consumption concerns of mobile devices. We wish to minimize the utilization of this channel.

$\mathcal{T} \leftrightarrow \mathcal{C}$ : As $\mathcal{T}$ is held locally by $\mathcal{C}$, this is a cheap channel (both in terms of bandwidth and power consumption), suitable for transmission of data linear in the size of $f$. 
$\mathcal{T} \leftrightarrow \mathcal{S}$ : There is no direct channel between $\mathcal{T}$ and $\mathcal{S}$, but, of course, $\mathcal{C}$ can pass (and potentially interfere with) messages between $\mathcal{T}$ and $\mathcal{S}$.

Trust. $\mathcal{C} \leftrightarrow \mathcal{S}$ : As in the standard SFE scenario, $\mathcal{C}$ and $\mathcal{S}$ don't trust each other. We address semi-honest, covert, and malicious $\mathcal{C}$ and $\mathcal{S}$.

$\mathcal{S} \leftrightarrow \mathcal{T}: \mathcal{T}$ is fully trusted by $\mathcal{S}$, since $\mathcal{T}$ is tamper-proof. $\mathcal{S}$ and $\mathcal{T}$ share a secret key $k$, used to establish a secure channel and to derive joint randomness.

$\mathcal{T} \leftrightarrow \mathcal{C}: \mathcal{C}$ does not trust $\mathcal{T}$, as $\mathcal{T}$ is the agent of $\mathcal{S}$, and may communicate with $\mathcal{S}$ through covert channels.

Storage, computation and execution. $\mathcal{C}$ and $\mathcal{S}$ are computationally strong devices which can perform both symmetric- and asymmetric-key operations ${ }^{3}$ Both have sufficient memory, linear in the size of $f . \mathcal{C}$ has control over $\mathcal{T}$, and can reset it, e.g., by interrupting its power supply. As justified in $\$ 4.1 .1, \mathcal{T}$ is a cheap special purpose $\mathrm{HW}$ with minimum chip surface: $\mathcal{T}$ has circuitry only for evaluating symmetric-key primitives in HW (no public-key or true random number generator) and has a small secure RAM. It may (\$4.1.4.3) or may not (\$4.1.4.4) have small non-volatile secure storage $\epsilon^{4}$, unaffected by the resets by $\mathcal{C}$.

\subsubsection{Token-Assisted SFE}

We start with a high-level overview of our protocols (\$4.1.4.1) and present the technical details of our construction afterwards - efficient circuit representation (\$4.1.4.2), and $\mathrm{GC}$ generation by stateful (\$4.1.4.3) and stateless tokens (\$4.1.4.4). Finally, we give implementation results in $\$ 4.1 .4 .5$.

\subsubsection{Protocols Overview}

Our constructions are a natural (but technically involved) modification of the software $(\mathrm{SW})$-based SFE protocols described in $\$ 2.3 .1$ that split the actions of the server into two parts - now executed by $\mathcal{S}$ and $\mathcal{T}$ - while maintaining provable security. We offload most of the work (notably, GC generation) to $\mathcal{T}$, thus achieving important communication savings, and partially offloading $\mathcal{S}$ 's computation to $\mathcal{T}$.

We start our discussion with the solution in the semi-honest model. However, as our modifications of the basic algorithms for $\mathrm{GC}$ creation are secure against malicious actions, our protocols are easily and efficiently extendible to covert and malicious settings.

At the high level, our token-assisted glsSFE protocols work as shown in Fig. 4.2: $\mathcal{C}$ obtains the garbled inputs $\widetilde{x}, \widetilde{y}$ from $\mathcal{S}$, and the GC $\widetilde{f}$ corresponding to the function $f$ from $\mathcal{T}$. Then, $\mathcal{C}$ evaluates $\tilde{f}$ on $\widetilde{x}, \widetilde{y}$ and obtains the result $z=f(x, y)$.

\footnotetext{
${ }^{3}$ If needed, $\mathcal{C}$ 's capabilities may be enhanced by using a $\mathrm{HW}$ accelerator as described in $\$ 4.2 .3$

${ }^{4} \mathcal{T}$ 's key $k$ is a fixed part of its circuit, and is kept even without non-volatile storage.
} 


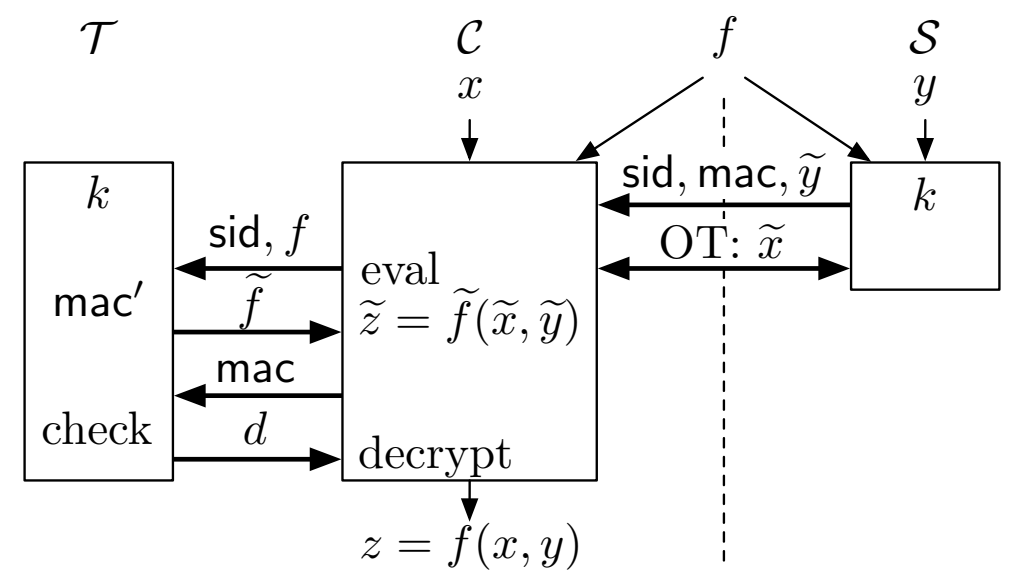

Figure 4.2: Protocols Overview: Token-Assisted SFE

It is easy to see that the introduction of $\mathcal{T}$ and offloading to it some of the computation does not strengthen $\mathcal{S}$, and thus does not bring security concerns for $\mathcal{C}$ (as compared to standard two-party SFE). On the other hand, separating the states of $\mathcal{S}$ and $\mathcal{T}$, placing $\mathcal{C}$ in control of their communication, and $\mathcal{C}$ 's ability to reset $\mathcal{T}$ introduces attack opportunities for $\mathcal{C}$. We show how to address these issues with the proper synchronization and checks performed by $\mathcal{S}$ and $\mathcal{T}$.

Our main tool is the use of a unique session id sid for each GC evaluation. From sid and the shared secret key $k, \mathcal{S}$ and $\mathcal{T}$ securely derive a session key $K$, which is then used to derive the randomness used in $\mathrm{GC}$ generation. We emphasize that each token shares a different random key $k$ with $\mathcal{S}$ to obtain unique session keys $K$ and to avoid break-one-break all behavior of multi-token systems. Jumping ahead (details in $\$ 4.1 .4 .3$, we note that sid uniqueness is easily achieved if $\mathcal{T}$ is stateful simply by setting sid equal to the value of a strictly monotonic session counter ctr maintained by $\mathcal{T}$. However, if $\mathcal{T}$ is stateless, $\mathcal{C}$ can always replay $\mathcal{S}$ 's messages. In $\$ 4.1 .4$.4 we show how to ensure that replays do not help $\mathcal{C}$.

Since $\mathcal{S}$ and $\mathcal{T}$ derive the same randomness for each session, the (same) GC $\tilde{f}$ can be generated by $\mathcal{T}$. Unfortunately, the weak $\mathcal{T}$ cannot store the entire function $f$. Instead, $\mathcal{C}$ provides the circuit corresponding to function $f$ gate-by-gate to $\mathcal{T}$, and obtains the corresponding garbled gate of $\widetilde{f}$. The garbled gate can immediately be evaluated by $\mathcal{C}$ and needs not to be stored. $\mathcal{C}$ is prevented from providing a wrong $f$ to $\mathcal{T}$, as follows. First, $\mathcal{S}$ issues a Message Authentication Code (MAC) of $f$, e.g., mac $=\operatorname{MAC}_{k}($ sid, $f)$, where $f$ is the agreed circuit representation of the evaluated function (cf. $\$ 4.1 .4 .2$ ). Further, $\mathcal{T}$ computes its version of the above MAC, $\mathrm{mac}^{\prime}$, as it answers $\mathcal{C}$ 's queries in computing $\tilde{f}$. Finally, $\mathcal{T}$ reveals the decryption information $d$ that allows $\mathcal{C}$ to decrypt the output wires only if $\mathcal{C}$ provides the matching mac. 
Garbled Inputs. The garbled input $\widetilde{y}$ of $\mathcal{S}$ can be computed by $\mathcal{S}$ and sent to $\mathcal{C}$, requiring $|y| \cdot(t+1)$ bits communication, where $t$ is the symmetric security parameter (cf. $\$ 2.1 .1 .2$ ). Alternatively, if $\mathcal{T}$ is stateful, $\mathcal{S}$ can establish a secure channel with $\mathcal{T}$, e.g., based on session key $K$, send $y$ over the channel, and have $\mathcal{T}$ output $\widetilde{y}$ to $\mathcal{C}$. This achieves the optimal communication between $\mathcal{S}$ and $\mathcal{C}$ of $\Theta(|y|)$ bits.

The garbled input $\widetilde{x}$ corresponding to $\mathcal{C}$ 's input $x$ can be transferred from $\mathcal{S}$ to $\mathcal{C}$ with a parallel OT protocol (cf. $\$ 2.2 .3$ ).

Extension to Covert and Malicious Parties. As described in \$2.3.1.2, standard $\mathrm{GC}$ protocols secure against covert or malicious adversaries employ cut-and-choose over multiple GCs $\widetilde{f}_{i}$ derived from seeds $s_{i}$.

These protocols similarly benefit from our separation of the server into $\mathcal{S}$ and $\mathcal{T}$. As in the semi-honest protocol, the GC generation can be naturally offloaded to $\mathcal{T}$, achieving corresponding computation and communication relief on the server and network resources. $\mathrm{GC}$ correctness verification is achieved by requesting $\mathcal{S}$ to reveal the generator seeds of the circuits to be opened. (Of course, these "opened" circuits are not evaluated.) Note that requirements on $\mathcal{T}$ are the same as in the semi-honest setting. Further, in both covert and malicious cases, the communication between $\mathcal{C}$ and $\mathcal{S}$ is independent of the size of $f$. The resulting communication complexity of these protocols is summarized in Table 4.2 .

Table 4.2: Communication Complexity: SW-based vs. Token-assisted SFE: Communication between server $\mathcal{S}$ and client $\mathcal{C}$ for secure evaluation of function $f$ with $n=|x|+|y|$ inputs, statistical security parameter $s$, and deterrence probability $1-1 / r$.

\begin{tabular}{|c|c|c|c|c|}
\hline Security & SW & based & SFE & Token-assisted SFE \\
\hline semi-honest & [Yao86] & $\overline{\overline{\mathcal{O}}(\mid f}$ & 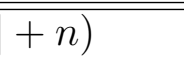 & $\overline{\mathcal{O}(n)}$ \\
\hline covert & GMS08 & $\mathcal{O}(\mid f$ & $+s n+r)$ & $\mathcal{O}(s n+r)$ \\
\hline malicious & [LP07] & $\mathcal{O}(s)$ & $\left.f \mid+s^{2} n\right)$ & $\mathcal{O}\left(s^{2} n\right)$ \\
\hline
\end{tabular}

Security. We present our protocols implicitly, by describing the modifications to the base protocols of [KS08a. We informally argue the security of the modifications as they are described. Formal proofs can be naturally built from proofs of [KS08a] and our security arguments. At the very high level, security against $\mathcal{S} / \mathcal{T}$ follows from the underlying $\mathrm{GC}$ protocols, since $\mathcal{S}$ is not stronger here than in the two-party SFE setting. The additional power of $\mathcal{C}$ to control the channel between $\mathcal{S}$ and stateful $\mathcal{T}$ is negated by establishing a secure channel $(4.1 .4 .3)$. C $\mathcal{C}$ 's power to reset stateless $\mathcal{T}$ is 
addressed by ensuring that by replaying old messages $\mathcal{C}$ gets either what she already knows, or completely unrelated data (\$4.1.4.4).

\subsubsection{Circuit Representation}

We now describe our circuit representation format. Our criteria are compactness, the ability to accommodate free XOR gates of [KS08a], and the ability of $\mathcal{T}$ to process the encoding "online", i.e., with small constant-size memory. Recall, $\mathcal{T}$ operates in request-response fashion: $\mathcal{C}$ incrementally, gate-by-gate, "feeds" the circuit description to $\mathcal{T}$ which responds with the corresponding garbled tables.

We consider circuits with two-input boolean gates. We note that our techniques can be naturally generalized to general circuits. A circuit can efficiently be transformed into our format as described below.

Token-friendly circuit format. Our format for representing the circuit in a tokenfriendly way is derived from standard circuit representations, such as that used in SFE tools like Fairplay [MNPS04 or TASTY (cf. \$5.2). For readability, we describe the format using a simple example circuit shown in Fig. 4.3. This circuit computes $z_{1}=x_{1} \wedge\left(y_{1} \oplus y_{2}\right)$, where $x_{1}$ is the input bit of $\mathcal{C}$ and $y_{1}, y_{2}$ are two input bits of $\mathcal{S}$. The corresponding circuit representation shown on the right is composed from the description of the inputs, gates, and outputs as follows.

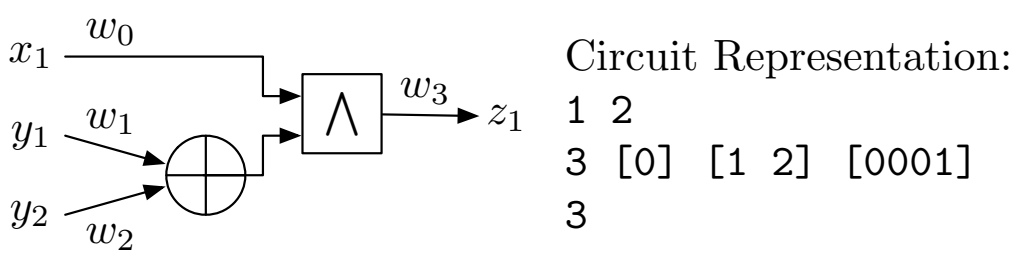

Figure 4.3: Example: Circuit Representation

Inputs and wires: The wires $w_{i}$ of the circuit are labeled with their index $i=$ $\{0,1, \ldots\}$. The first $u=|x|$ wires are associated with the input of $\mathcal{C}$, the following $v=|y|$ wires are associated with the input of $\mathcal{S}$, and the internal wires are labeled in topological order starting from index $u+v$ (output wires of XOR gates are not labeled, as XOR gates are incorporated into their successor gates as described in the next paragraph). The first line of the circuit description specifies $u$ and $v$ (Fig. 4.3. $u=1, v=2$ ).

Gates are labeled with the index of their outgoing wire; each gate description specifies its input wires. XOR gates do not have gate tables and are omitted from the description. Rather, non-XOR gates, instead of pointing to two input wires, include two input wire lists. If the input list contains more than one wire, these wire values are to be XORed to obtain the corresponding gate input. Gate's description concludes 
with its truth table. In Fig. 4.3, the second line describes the AND gate, which has index 3 , and inputs $w_{0}$ and $w_{1} \oplus w_{2}$.

Outputs: The circuit description concludes with $|z|$ lines which contain the indices of the output wires (Fig. 4.3 , the only $(|z|=1)$ output wire is $\left.w_{3}\right)$.

Large XOR sub-circuits. In our token-friendly circuit format, XOR gates with fan-out $>1$ occur multiple times in the description of their successor gates. In the worst case, this results in a quadratic increase of the circuit description. To avoid this cost, a (non-XOR) identity gate with arbitrary fanout can be inserted after trees of $\mathrm{XOR}$ gates.

Conversion into token-friendly circuit format. In order to convert a given circuit with $d$-input gates into our token-friendly circuit format described above, the gates are first decomposed into 2-input gates and afterwards the XOR gates are grouped together:

Decomposition into 2-input gates. Decomposing the $d$-input gates of a circuit into multiple 2-input gates can be done in a straight-forward way using Shannon's expansion theorem [Sha49] or the Quine-McCluskey algorithm which results in smaller circuits [Tur96]. For small $d$ (e.g., for the very common case of $d=3$ ), the optimal replacement can be found via brute-force enumeration of all possibilities (cf. \$3.2.2).

Grouping of XOR gates. The XOR gates can be grouped together as follows: To each input wire and each output wire of a non-XOR gate $i$ we assign the set $\{i\}$. Afterwards we transfer the gates of the circuit in topological order and annotate to the output wire of each XOR gate the following set which is computed from the sets of its input wires $S_{1}, S_{2}$ as $S=S_{1} \oplus S_{2}:=\left(S_{1} \cup S_{2}\right) \backslash\left(S_{1} \cap S_{2}\right)$. Finally, the remaining non-XOR gates are output in the token format using the sets associated to the input wires which contain those wires which need to be XORed together for the specific input. As merging two sets of size at most $|f|$ entries each can be done in $\mathcal{O}(|f|)$ operations, the overall complexity of this algorithm is in $\mathcal{O}\left(|f|^{2}\right)$.

\subsubsection{GC Creation with Stateful Token Using Secure Counter}

The main idea of our GC generation with small RAM footprint is having $\mathcal{T}$ generate garbled tables "on the fly". This is possible, since for each non-XOR gate, the garbled table can be generated given only the garbled values of the gate's input and output wires. In our implementation, we pseudorandomly derive these garbled values from the session key and their wire indices. The rest of this section contains further details.

Session Initialization. SFE proceeds in sessions, where one session is used to securely evaluate a function once. $\mathcal{T}$ has a secure monotonic session counter ctr which is (irreversibly) incremented at the beginning of each session. The session id sid is set to the incremented state of ctr. (We omit the discussion of re-synchronization of ctr between $\mathcal{T}$ and $\mathcal{S}$ which may be needed due to communication and other errors.) Then, 
the session key is computed by $\mathcal{S}$ and $\mathcal{T}$ as $K=\mathrm{PRF}_{k}($ sid) and subsequently used to provide fresh randomness to create the $\mathrm{GC}$.

As required by the construction of [KS08a] (cf. 2.2.2.3), the two garbled values of the same wire differ by a global difference offset $\Delta$. This offset is derived from $K$ at session initialization and kept in RAM throughout the session.

Subsequently, the garbled values for wire $w_{i}$ are derived on-the-fly from $K$ as

$$
\widetilde{w}_{i}^{0}=\operatorname{PRF}_{K}(i), \quad \widetilde{w}_{i}^{1}=\widetilde{w}_{i}^{0} \oplus \Delta .
$$

Garbled Gates. $\mathcal{T}$ receives the description of the circuit, line by line, in the format described in $\$ 4.1 .4 .2$, and generates and outputs to $\mathcal{C}$ corresponding garbled gates, using only small constant-size memory. $\mathcal{T}$ first verifies that the gate with the same label had not been processed before. (Otherwise, by submitting different gate tables for the same gate, $\mathcal{C}$ may learn the real wire values). This is achieved with the monotonically increasing processed gate counter gctr, verifying that gate's label glabel $>$ gctr, and setting gctr $=$ glabel. $\mathcal{T}$ then derives and stores the garbled values of the gate's input and output wires according to Equation 4.1). (For input lists, the wire's garbled value $\widetilde{w}^{0}$ is computed as the XOR of the garbled values of the listed wires, and $\widetilde{w}^{1}$ is set to $\widetilde{w}^{0} \oplus \Delta$. Note that this requires constant $\mathrm{RAM}$.) Finally, based on these garbled values, the garbled table of the gate is computed and output to $\mathcal{C}$.

Garbled Outputs. Recall, $\mathcal{T}$ must verify circuit correctness by checking mac generated by $\mathcal{S}$. Thus, $\mathcal{T}$ does not release the output decryption tables to $\mathcal{C}$ until after the successful check. At the same time, the check is not complete until the entire circuit had been fed to $\mathcal{T}$. To avoid having $\mathcal{T}$ store the output decryption tables or involving $\mathcal{S}$ at this stage, $\mathcal{T}$ simply encrypts the output tables using a fresh key $K^{\prime}$, and outputs the key only upon a successful MAC verification.

\subsubsection{GC Creation with Stateless Token (no Counter)}

As discussed above, while non-volatile secure storage (the session counter ctr) is essential in our protocol of $\$ 4.1 .4 .3$, in some cases, it may be desired to avoid its cost. We now discuss the protocol amendments required to maintain security of SFE with the support of a token whose state can be reset by $\mathcal{C}$, e.g., via power interruption.

First, we observe that $\mathcal{S}$ is still able to maintain state, and choose unique session ids sid. However, $\mathcal{T}$ can no longer be assured that sid claimed by $\mathcal{C}$ is indeed fresh. Further, $\mathcal{T}$ does not have a source of independent randomness, and thus cannot establish a secure channel with $\mathcal{S}$, e.g., by running a key exchange.

We begin with briefly describing a replay vulnerability of our protocol of \$4.1.4.3, when $\mathcal{T}$ is executed with the same sid. In one session, $\mathcal{C}$ properly executes SFE. In another session, $\mathcal{C}$ runs $\mathcal{T}$ with the same sid, but feeds $\mathcal{T}$ an incorrect circuit, receiving valid garbled tables for each of the gates, generated for the same garbled wire values. Now, even though $\mathcal{T}$ will not accept mac and will not decrypt the output wires, $\mathcal{C}$ had already received them in the first execution. It is easy to see that $\mathcal{C}$ "wins". 
Our solution is to ensure that $\mathcal{C}$ does not benefit from replaying the same session id sid twice to $\mathcal{T}$. To achieve this, we require that each garbled value depends on the (hash of the) entire circuit on which this wire depends, as described below. If $\mathcal{C}$ replays a previously used sid with a different circuit, she will not be able to relate the produced garbled table with a previous output of $\mathcal{T}$.

We associate with each wire $w_{i}$ a (revealed to $\mathcal{C}$ ) hash value $h_{i}$. For input wires, $h_{i}$ is the empty string. For each other wire $w_{i}, h_{i}$ is derived (e.g., via a cryptographic hash function $H$ such as $\mathrm{SHA}-256$ modeled as Random Oracle (RO) from the description of the gate $g_{i}$ (which includes its index $i$, truth table $T_{i}$, and list of inputs; cf. \$4.1.4.2) that emits that wire: $h_{i}=H(\langle$ gate_description $\rangle)$. The garbled value of wire $w_{i}$ now depends on its hash value $h_{i}: \widetilde{w}_{i}^{0}=\operatorname{PRF}_{K}\left(h_{i}\right)$ and $\widetilde{w}_{i}^{1}=\widetilde{w}_{i}^{0} \oplus \Delta$. Finally, to enable the computation of the garbled tables, $\mathcal{C}$ must feed back to $\mathcal{T}$ the hashes $h_{i}$ of the input wires, and receive from $\mathcal{T}$ and keep for future use the hash of the output wire. As noted above, $\mathcal{C}$ 's attempts to feed incorrect values result in the output of garbled tables that are unrelated to previous outputs of $\mathcal{T}$, and thus do not help $\mathcal{C}$.

We note that this construction for stateless tokens can be used together with the Garbled Row Reduction GC technique of [NP01] to remove one entry from each garbled table (cf. $\$ 2.2 .2 .3$ ).

\subsubsection{Hardware Implementation}

For the token-assisted GC creation method with stateful tokens described in 84.1 .4 .3 , a $\mathrm{HW}$ architecture for an FPGA has been designed and implemented in VHDL. As described in [JKSS10a], the $\mathrm{HW}$ requirements are moderate and dominated by AES cores to implement PRF and MAC, and a SHA-256 core to implement $H$. We summarize and compare the performance of the prototype implementation with previous SW based solutions next.

Overall, the latency of the $\mathrm{HW}$ implementation, determined with ModelSim, is \#clock_cycles $=158 G_{1}+312 G_{2}+154 O+150$, where $G_{1}, G_{2}$ is the number of 1 -input gates respectively 2 -input gates and $O$ is the number of outputs.

Example 6. The $H W$ implementation generates a $G C$ for our optimized 16-bit comparison circuit of $\$ 3.3 .3 .1\left(G_{1}=0, G_{2}=16, O=1\right)$ in 5,296 clock cycles $(\approx 80 \mu \mathrm{s}$ with $50 \mathrm{MHz}$ clock). In $S W$, this takes roughly $0.5 \mathrm{~s}$ on an Intel Core 26420 at $2.13 \mathrm{GHz}$ [LPS08].

Example 7. Generating a $G C$ for AES-128 encryption $\left(G_{1}=12614, G_{2}=11334\right.$, $O=128$ ) takes $5,549,082$ clock cycles ( $\approx 84 \mathrm{~ms}$ with $50 \mathrm{MHz}$ clock). In $S W$, this takes approximately $1 \mathrm{~s}$ on an Intel Core 2 Duo at $3.0 \mathrm{GHz}$ [PSSW09].

We note that the optimization for grouping large XOR sub-circuits described in the end of $\$ 4.1 .4 .2$ dramatically reduces the amount of communication between $\mathcal{C}$ and $\mathcal{T}$ : When using this optimization, the size of the AES circuit of Example 7 is $|f|=1.1 \mathrm{MB}$ 
and the garbled AES circuit has size $|\widetilde{f}|=1.1 \mathrm{MB}$. Without this optimization, the circuit has no more 1-input gates $\left(G_{1}=0\right)$ which results in a faster creation $(3,556,070$ clock cycles), evaluation, and smaller size of the $\mathrm{GC}(|\widetilde{f}| \approx 0.7 \mathrm{MB})$; however, as the lists of inputs to be XORed must be repeated for each use, the size of the circuit would be drastically larger $(|f|=94.5 \mathrm{MB})$ which might be a bottleneck if the communication between $\mathcal{C}$ and $\mathcal{T}$ is slow.

\subsection{One-Time Programs}

In this section we investigate the practicality of One-Time Programs (OTPs), a noninteractive version of Garbled Circuit (GC)-based Secure Function Evaluation (SFE) protocols which is able to provably protect against arbitrary side-channel leakage.

We propose an efficiency improvement for OTPs with multiple outputs (\$4.2.2.2) and describe a generic architecture for using OTPs in a modular way to protect against arbitrary side-channel attacks in $\$ 4.2 .2 .3$. Further, we present a hardware (HW) architecture (\$4.2.3.2) and optimizations (\$4.2.3.3) for efficient evaluation of GC/OTP on memory-constrained embedded systems. Implementation results of our architecture and optimizations on a Field-Programmable Gate Array (FPGA) (4.2.3.4) show that provable leakage-resilience via OTP comes at a relatively high cost, but its use might still be justified in high-security applications.

Publication Info: Parts of the following results are published in [JKSS10c.

\subsubsection{Motivation}

In the following we summarize side-channels and protection mechanisms (\$4.2.1.1), motivate how $\mathrm{HW}$-assisted SFE can protect against leakage $(\$ 4.2 .1 .2)$, and give the objectives of this section $(\$ 4.2 .1 .3$.

\subsubsection{Side-Channels and Protection}

For over a decade, we saw the power and elegance of side-channel attacks on a variety of cryptographic implementations and devices. These attacks refute the assumption of "black-box" execution of cryptographic algorithms, allow the adversary to obtain (unintended) internal state information, such as secret keys, and consequently cause catastrophic failures of the systems. Often the attacks are on the device in attacker's possession, and exploit physical side-channels such as observing power consumption [KJJ99], emitted radiation GMO01, QS01, AARR02, and even the memory cache [KSWH98, Pag02, OST06]. Moreover, even when no computation is performed, stored secrets may be leaked [Sko05] or read out from Random Access Memory (RAM), which is typically not erased at power-off, allowing, e.g., cold-boot attacks $\left[\mathrm{HSH}^{+} 08\right]$. Hence, 
from the $\mathrm{HW}$ perspective, security has been viewed as more than the algorithmic soundness in the black-box execution model (see, e.g., [Lem06, Wei00, VS07, Smi03]).

Today's practical countermeasures typically address known vulnerabilities, and thus target not all, but specific classes of side-channel attacks. The desire for a complete solution motivated the recent burst of theoretical research in leakage-resilient cryptography, the area that aims to define security models and frameworks that capture leakage aspects of computation or/and memory. Information leakage is typically modeled by allowing the adversary to learn (partial) memory or execution states. The exact information given to the adversary is specified by the (adversarially chosen) leakage function. Then, the assumption on the function (today, usually the bound on the output length) directly translates into a physical assumption on the underlying device and the adversary. Proving security against such an adversary implies security in the real-world with the real device, subject to corresponding assumption (see Pie09] for a survey on this strand of research). We note that many of the results of this new line of research (i.e., leakage assumptions and leakage-resilient constructions), although clearly stated, have not yet been evaluated by practitioners and side-channel community $!^{5}$ Further, efficiency is a major concern with today's solutions, since, e.g., embedded systems on an integrated circuit (IC) have very little cost tolerance. 6

\subsubsection{SFE in Hardware and Leakage-Resilience}

Efficient SFE in an untrusted environment is a longstanding objective of modern cryptography. Informally, the goal of two-party SFE is to let two mutually mistrusting (polynomially-bounded) parties compute an arbitrary function on their private inputs without revealing any information about the inputs, beyond the output of the function (cf. 2.3.1). SFE has a variety of applications, particularly in settings with strong security and privacy demands. Deployment of SFE was very limited and believed expensive until recent improvements in algorithms, code generation, computing platforms and networks.

Because of the execution flow of GC-based SFE (one party can non-interactively evaluate the function once the inputs have been fixed), the security guarantees of SFE are well-suited to prevent all side-channel leakage. Indeed, even GC evaluation in the open reveals no information other than the output. Clearly, it is safe to let the adversary see (as it turns out, even to modify) the entire GC evaluation process. The inputs-related stage of GC can also be made non-interactive with appropriate HW such as the (slightly extended) Trusted Platform Module (TPM) GT08. Goldwasser et al. GKR08] observed that very simple HW, called One-Time Memory (OTM), is sufficient, one that, hopefully, can be manufactured tamper-resistant at low cost.

\footnotetext{
${ }^{5}$ Indeed, ongoing work of $\mathrm{SPY}^{+} 09$ investigates the practical applicability and usability of theoretical leakage models and the constructions proven secure therein.

${ }^{6}$ At the same time, e.g., the size of private circuits in ISW03 grows quadratically with the number of wire probes by the adversary.
} 
They propose to use OTPs, a combination of GC and OTMs, for leakage-resilient computation. Indeed, one of our goals is to evaluate today's performance of OTP in HW.

\subsubsection{Our Objectives}

Practical efficiency of SFE and leakage-resilient computing is critical. Indeed, in most settings, the technology can only be adopted if its cost impact is acceptably low. We pursue the following two objectives.

First, we aim to mark this (practical efficiency) boundary by considering $H W$ accelerated state-of-the-art GC evaluation, and optimizing it for embedded systems. Implementing SFE (at least partially) in $\mathrm{HW}$ promises to significantly improve computation speed and reduce power consumption. We evaluate costs, benefits and trade-offs of HW support for GC evaluation.

Second, we use our GC HW-accelerator to implement OTP and evaluate the efficiency of this provably leakage-resilient protection. The envisioned applications for OTPs mentioned in [GKR08] are complex functionalities such as one-time proofs, E-cash, or extreme software protection (with features such as limited number of executions or temporary transfer of cryptographic abilities). However, the exact circuit sizes of these functions, and hence the OTP practicability for these applications, are not yet clear. We make a first step towards estimating the costs of such complex OTP applications by implementing OTP evaluation of the Advanced Encryption Standard (AES) function. We chose AES as it is relatively complex and allows comparison with existing (heuristic) leakage protection. OTP evaluation of AES can be used for sending a small number of messages securely over a completely untrusted platform (e.g., a computer in an Internet café) using a simple tamper-proof HW token (e.g., a USB token) and the same key for encrypting/authenticating multiple messages.

\subsubsection{Non-interactive GCs and One-Time Programs}

Using GCs for SFE, although traditionally considered in the interactive setting between server $\mathcal{S}$ and client $\mathcal{C}$ (cf. $\$ 2.3 .1 .1$ ), relies on interaction only as much as does the underlying Oblivious Transfer (OT) protocol. Consequently, as noted in [CCKM00, the round complexity and (non-)interactivity features of the $\mathrm{GC}$ protocol are exactly those of the underlying $\mathrm{OT}$. In the non-interactive setting, the server who generates the $\mathrm{GC}$ is called sender $\mathcal{S}$ and the client who non-interactively evaluates the GC is called receiver $\mathcal{R}$.

Related Work. The combination of GC with non-interactive OT in the semi-honest setting was proposed in GT08. For malicious receiver, OTP were introduced in GKR08 using minimal HW assumptions. A construction for non-interactive secure computation with information-theoretic security was given in [GIS ${ }^{+} 10$ ] using multiple 
HW tokens; [DKM11] give a construction with one HW token. [IKO ${ }^{+} 11$ ] consider non-interactive secure computation in the malicious model with black-box usage of a pseudorandom generator and a single round of parallel calls to an OT oracle.

We extend and implement OTP evaluation in HW. Our extension is in the computational model with Random Oracles (ROs), secure against malicious receiver, and more efficient than the constructions of [GKR08, $\left.\mathrm{GIS}^{+} 10\right]$.

\subsubsection{Previous Works}

In GT08, the authors suggested to extend the TPM [TCGT09] for implementing non-interactive OT, resulting in a non-interactive version of Yao's protocol in the semi-honest setting. Subsequently, One-Time Programs (OTPs) were introduced in GKR08]. This approach considers malicious receivers and can be viewed simply as Yao's GC protocol, where the OT function calls are implemented with OTM devices. An OTM $T_{i}$ is a simple tamper-proof $\mathrm{HW}$, which allows a single query of one of the two stored garbled values $\widetilde{x}_{i}^{0}, \widetilde{x}_{i}^{1}$ (GKR08] suggests using a tamper-proof one-timesettable bit $b_{i}$ which is set as soon as the OTM is queried).7 Further, OTM-based $\mathrm{GC}$ execution can be non-interactive, in the sense that the sender $\mathcal{S}$ can send the GC and corresponding OTMs to the receiver $\mathcal{R}$, who will be able to execute one instance of SFE on any input of her choice without further interaction with the sender. As also noted in [GIS ${ }^{+} 10$ ], the evaluated function can be fully hidden by evaluating a universal function instead. In practice, one would evaluate a garbled Universal Circuit that is programmed to compute the intended function (cf. \$2.3.1.3). Finally, GCs (and hence also OTPs) are inherently one-time execution objects (generalizable to $k$-time execution by repetition).

A subtle issue in this context, noted and addressed in GKR08], is the following. Previous GC-based solutions were either in the semi-honest model, or used interaction during protocol execution, which precluded the receiver $\mathcal{R}$ from choosing her inputs adaptively, based on the given (and even partially evaluated) GC. This possibility of adaptively chosen inputs results in possible real attacks by a malicious receiver $\mathcal{R}$ in the non-interactive setting 8 The solution of [GKR08] is to mask (each) output bit $z_{j}$ of the function with a random bit $m_{j}$, equal to the XOR of (additional) random bits $m_{i, j}$ contributed by each of the input OTMs $T_{i}$, i.e., $m_{j}=m_{1, j} \oplus m_{2, j} \oplus \ldots$ and $z_{j}^{\prime}=z_{j} \oplus m_{j}$. The real-world adversary does not learn the output of the function before it had queried all OTMs with its inputs, which precludes it from adaptively selecting the input? 9

\footnotetext{
${ }^{7}$ Indeed, this is one of the simplest functionalities possible, and one that is hopefully easier to protect against leakage and tampering (we refer the reader to GKR08 for extended discussion on such protection).

${ }^{8}$ From the mathematical perspective, the standard proof of security of $\mathrm{GC}$ now does not go through, since the simulator would have to output to $\mathcal{R}$ the simulated GC (i.e., its garbled tables and output wire decoding) before knowing the inputs of the malicious receiver.

${ }^{9}$ In the proof, the new simulator is able to produce an indistinguishable simulation, since it only
} 
In the following we present an efficiency improvement $(\$ 4.2 .2 .2$, and a generic architecture for leakage-resilient and tamper-proof computation derived from OTP $(\$ 4.2 \cdot 2.3)$.

\subsubsection{Extending One-Time Programs}

As mentioned in $\$ 4.2 .2 .1$, the solution in GKR08 requires each OTM token to additionally store a string of the size of the output. We propose a practical performance improvement to the technique proposed in GKR08, which is beneficial for OTP evaluation of functions with many output bits. In our solution each OTM token (in addition to the two garbled values) stores a random string $r_{i}$ of length of the symmetric security parameter $t$ (cf. $\$ 2.1 .1 .2$. Consequently, our construction results in smaller OTMs when the function to be evaluated has more outputs than $t$. As a trade off, our security proof utilizes ROs, as we do not immediately see how to avoid their use and have OTM size independent of the number of outputs (cf. \$2.1.5 for our discussion on the $\mathrm{ROs}$ model). To additionally allow $\mathcal{C}$ to check that the GC evaluation was performed correctly, we make use of the technique of [NPS99, Sect. 2.4] where $\mathcal{S}$ provides for each output wire a one-way image for each of the two possible garbled output values (cf. $\$ 2.3 .2$.

Our main idea is to insert a "hold off" gate into each output wire $W_{j}$ which can only be evaluated once all input OTMs had been queried, thus preventing malicious receiver $\mathcal{R}$ from choosing her inputs adaptively. It can be implemented by requiring a call to an ideal cipher $E$ (modeled as a $\mathrm{RO}$ and instantiated for example with AES) keyed with data from all OTMs ${ }^{10}$ To implement this, we secret-share a random $t$-bit value $r \in_{R}\{0,1\}^{t}$ over all OTMs, where $t$ is the symmetric security parameter (cf. 2.1.1.2). That is, OTM $T_{i}$ additionally stores a share $r_{i}$ (released to $\mathcal{R}$ with $\widetilde{x}_{i}$ upon the query), where $r=\bigoplus_{i} r_{i}$. Receiver $\mathcal{R}$ is able to recover $r$ if and only if she queried all OTMs.

Fig. 4.4(b) depicts our construction: Our version of OTM $T_{i}$, in addition to the two OT secrets $\widetilde{x}_{i}^{0}, \widetilde{x}_{i}^{1}$ and the tamper-proof bit $b_{i}$, contains a random share $r_{i} \in R\{0,1\}^{t}$ which is released together with $\widetilde{x}_{i}^{x_{i}}$ once $T_{i}$ is queried with input bit $x_{i}$. The GC is constructed as usual (e.g., as described in $\$ 2.2 .2$ ), with the following exception. On each output wire $W_{j}$ with garbled outputs $\widetilde{z}_{j}^{0}, \widetilde{z}_{j}^{1}$, we append a one-input, one-output OT-commit gate $G_{j}$, with no garbled table. We set the output wire secrets of $G_{j}$ to $\widehat{z}_{j}^{0}=E_{r}\left(\widetilde{z}_{j}^{0}\right), \widehat{z}_{j}^{1}=E_{r}\left(\widetilde{z}_{j}^{1}\right)$. To enable $\mathcal{R}$ to compute the wire output non-interactively, GC also specifies that $\widehat{z}_{j}^{b}$ corresponds to $b$.

Note that a malicious $\mathcal{R}$ is unable to complete the evaluation of any wire of GC until all the OTMs have been queried, and all her inputs have been specified. Further,

commits to the output values of the simulated GC when the last OTM is queried, the point at which the simulator knows the inputs of the malicious receiver.

${ }^{10}$ Alternatively, a hash function $H$ can be used which is less efficient [JKSS10c. 
she is not able to lie about the result of the computation, since she can only compute one of the two values $\widetilde{z}_{j}^{0}, \widetilde{z}_{j}^{1}$. Demonstration of knowledge of $\widetilde{z}_{j}^{i}$ serves as a proof for the corresponding output value (cf. $\$ 2.3 .2$ ).

Theorem 4. The above protocol is secure against a semi-honest sender $\mathcal{S}$, who generates the OTM tokens and the $G C$, and malicious receiver $\mathcal{R}$, in the $R O$ model.

Proof. Security against semi-honest $\mathcal{S}$ is trivial as $\mathcal{S}$ does not see $\mathcal{R}$ 's inputs (we consider OTMs a separate entity from $\mathcal{S}$ ).

We now describe the simulator Sim which will produce a view indistinguishable from the view of $\mathcal{R}$ in real execution. Sim will query the receiver $\mathcal{R}$ as a black box and answer all of $\mathcal{R}$ 's queries, including calls to (simulated) $\mathrm{RO} \mathcal{O}$. Our proof is based on the idea that Sim will "program" $\mathcal{O}$ such that the output of the "hold off" gates will match the output given by the trusted party of the ideal game.

Without loss of generality, we assume that $\mathcal{R}$ queries RO only once for each distinct input. Upon initialization, Sim constructs GC, as would an honest $\mathcal{S}$ in the construction described above, and sends the GC to $\mathcal{R}$ together with randomly chosen commitments $\widehat{z}_{j}^{0}, \widehat{z}_{j}^{1}$ for all output wires. Additionally, Sim generates a random key $r$ and a random secret sharing $r=\bigoplus_{i} r_{i}$ of it. For the wires corresponding to the input of $\mathcal{S}, \operatorname{Sim}$ sends secrets corresponding to 0 -values. Whenever $\mathcal{R}$ queries the $i$-th OTM with input bit $x_{i}$, Sim responds with the corresponding garbled value $\widetilde{x}_{i}^{x_{i}}$, constructed earlier as part of the GC construction, and the share $r_{i}$. Once $\mathcal{R}$ had queried the final OTM, Sim sends the input received from $\mathcal{R}$ to the trusted party, and receives the output $f(x, y)$ of the computation. Now Sim "programs" $\mathcal{O}$ to output certain values according to the received $f(x, y)$. That is, on input $\left(r, \widetilde{z}_{j}\right)$ (call associated with OT-commit gate $G_{i}$ and the $j$-th bit of the output), $\mathcal{O}$ will output $\widehat{z}_{j}^{f_{j}(x, y)}$, i.e., the commitment for the wire leaving $G_{j}$ that corresponds to the bit $f_{j}(x, y)$ of the output it received from the trusted party.

It is not hard to see that the above simulator generates a view indistinguishable from the view of $\mathcal{R}$ in the real execution. First, we note that the simulated GC and responses to RO and OTM queries are indistinguishable from the real execution. Thus, in particular, $\mathcal{R}$ "behaves normally" during the simulation, and would not be able to, e.g., substitute inputs in a special way, etc. Further, "programming" of $\mathcal{O}$ will not be noticed by $\mathcal{R}$, since she can query programmed values only with negligible probability prior to completing all OTM calls (since $r$ is random and unknown to $\mathcal{R}$ prior to completing all OTM calls).

\subsubsection{Architecture for Using One-Time Programs}

Most of today's countermeasures to side-channel attacks are specific to known attacks. Protecting HW implementations (e.g., of AES) usually proceeds as follows (e.g., see [AG01]). First, the inputs are hidden, typically by applying a random mask (this 
requires trusted operation, and often the corresponding assumption is introduced). Afterwards, the computation is performed on the masked data. To allow this, the functionality needs to be adapted (e.g., using amended AES S-boxes). Finally, the mask is taken off to reveal the output of the computation.

As shown in Fig. 4.4(a), we use a similar approach with similar assumptions to provably protect arbitrary functionalities against all attacks, both known and unknown:

1. The private data $x$ provided by $\mathcal{R}$ is masked in a trusted environment MASK. The masked data $\widetilde{x}$ does not reveal any information about the private data, but still allows to compute on it.

2. The computation on the masked data is performed in an untrusted environment where the adversary is able to arbitrarily interfere (passively and actively) with the computation. To compute on the masked data, the evaluation algorithm EVAL needs a specially masked version of the program $\widetilde{P}$. Additionally, $\mathcal{S}$ can provide masked inputs $\widetilde{y}$ to parametrize the program. The result of EVAL is the masked output $\widetilde{z}$.

3. Finally, $\widetilde{z}$ is unmasked into the plain output $z$. The procedure UNMASK allows to verify that $\widetilde{z}$ was computed correctly, i.e., no tampering happened in the EVAL phase in which case UNMASK outputs the failure symbol $\perp$. For correctness of this verification, UNMASK is executed in a trusted environment where the adversary can observe but not modify the computation.

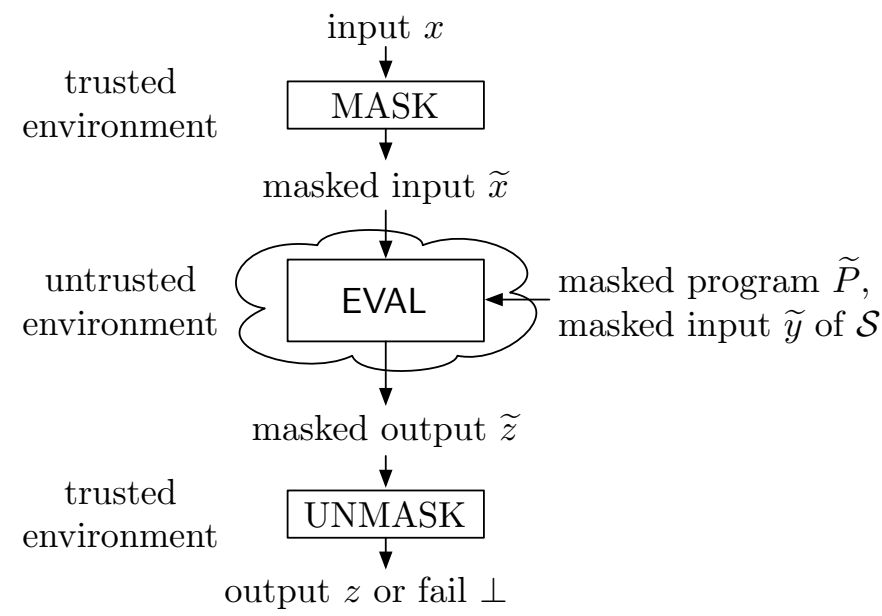

(a) Generic Architecture

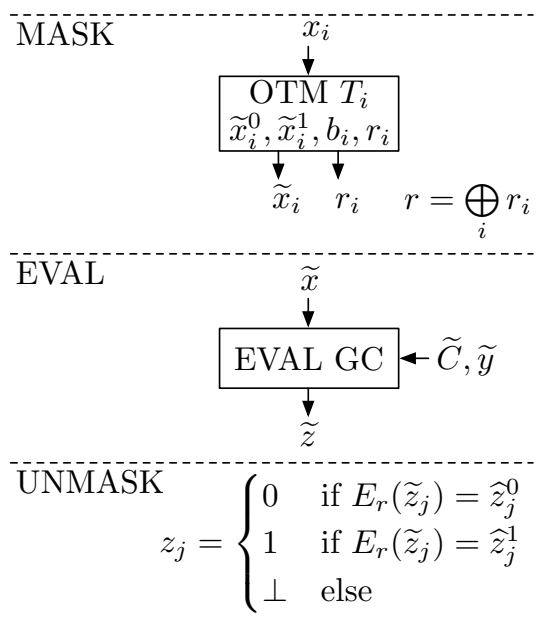

(b) Using One-Time Memory

Figure 4.4: Evaluating a Functionality without Leakage 
More specifically, the masked program $\widetilde{P}$ is a $\mathrm{GC} \widetilde{C}$, masked values $\widetilde{x}, \widetilde{y}, \widetilde{z}$ are garbled values and the algorithms MASK, EVAL and UNMASK can be implemented as described next.

MASK: Masking the input data $x$ of receiver $\mathcal{R}$ is performed by mapping each bit $x_{i}$ to its corresponding garbled value $\widetilde{x}_{i}=\widetilde{x}_{i}^{x_{i}}$. This can be provided externally (e.g., by interaction with a party on the network). We concentrate on on-board non-interactive masking which requires certain $\mathrm{HW}$ assumptions (see below). The following can be directly used as a (non-interactive) MASK procedure:

- OTMs GKR08: For small functionalities, we favor the very cheap One-Time Memorys (OTMs), as this seems to carry the weakest assumptions (cf. \$4.2). However, as OTMs can be used only once, a fresh OTM must be provided for each evaluation and each input bit. For practical applications, OTMs (together with their GC) could be implemented for example on a tamper-proof USB token for easy distribution.

- Non-interactive OT, Trusted Platform Modules (TPMs) are low-cost tamperproof cryptographic chips embedded in many of today's PCs [TCGT09]. TPM masking based on the non-interactive Oblivious Transfer (OT) protocol of [GT08] requires the (slightly extended) TPM to perform asymmetric cryptographic operations in form of a count-limited private key whose number of usages is restricted by the TPM chip. An interactive protocol allows re-initialization for future non-interactive OTs instead of shipping new HW. An alternative approach would be the recently proposed efficient non-interactive OT protocol based on cheap tamper-proof $\mathrm{HW}$ tokens that compute symmetric cryptographic operations only [DSV10].

- Smartcard: In our preferred solution for larger functionalities, masking could be performed by a tamper-proof smartcard. The smartcard would keep a secure monotonic counter to ensure a single query per input bit. Another advantage of this approach is that the same smartcard can be used to generate the GC as well, thus eliminating $\mathrm{GC}$ transfer over the network as described in $\$ 4.1$. Further, the smartcard can be naturally used for multiple OTP evaluations.

For non-interactive masking, the $\mathrm{HW}$ that masks the inputs must be trusted and the entire input must be specified before anything about the output $z$ is revealed to prevent adaptive input selection as discussed in 4.2 .2 .1 and $\$ 4.2 .2 .2$.

EVAL: The implementation of EVAL (of the masked program $\widetilde{P}$ on masked inputs $\widetilde{x}$ and $\widetilde{y}$ ) in embedded systems is presented in detail in $\$ 4.2 .3$. Here we note that $\widetilde{P}$ and $\widetilde{y}$ (masked input of $\mathcal{S}$ ) can be generated offline by the semi-honest sender $\mathcal{S}$ and 
provided to EVAL by convenient means (e.g., via a data network or a storage medium). This is the scenario advocated in [GKR08]; one of its advantages is that generation of $\widetilde{P}$ does not leak to EVAL. Alternatively, $\widetilde{P}$ and $\widetilde{y}$ could be generated "on-the-fly" using a cheap, simple, constant-memory, stateless, and tamper-proof token as shown in $\$ 4.1$. We reiterate that the masked program $\widetilde{P}$ can be evaluated exactly once.

UNMASK: Finally, the masked output $\widetilde{z}$ is checked for correctness and decoded non-interactively by $\mathcal{R}$ into the plain output $z$ as follows (cf. 4.2 .2 .2 and Fig. 4.4(b)) For each output wire, the masked program $\widetilde{P}$ specifies the correspondence $\widehat{z}_{j} \rightarrow z_{j}$ in form of the two valid encryptions $\widehat{z}_{j}^{0}$ and $\widehat{z}_{j}^{1}$. Even if EVAL is executed in a completely untrusted environment (e.g., processed on untrusted HW), its correct execution can be verified efficiently: when $E_{r}\left(\widetilde{z}_{j}\right)$ is neither $\widehat{z}_{j}^{0}$ nor $\widehat{z}_{j}^{1}$ the garbled output $\widetilde{z}_{j}$ is invalid and UNMASK outputs the failure symbol $\perp$. The reason for this verifiability property of $\mathrm{GC}$ is that a valid garbled output $\widetilde{z}_{j}$ can only be obtained by correctly evaluating the GC but cannot be guessed as observed in [NPS99, Sect. 2.4] (cf. \$2.3.2).

\subsubsection{Fully Homomorphic Encryption against Leakage}

At the first glance, the recently proposed fully Homomorphic Encryption (HE) schemes described in $\$ 2.2 .1 .2$ may seem as an attractive alternative solution for leakage-free computation. Indeed, fully $\mathrm{HE}$ allows to compute arbitrary functions on encrypted data without the need for helper data in form of a masked program. The MASK algorithm would homomorphically encrypt the input $x$ and the UNMASK algorithm could decrypt the result. Using verifiable computation [GGP10] (cf. \$4.3.3.2), fully $\mathrm{HE}$ can also be extended to allow verification that the computation was performed correctly.

However, we argue that fully $\mathrm{HE}$ is in fact not appropriate in our setting: Our first comment, which concerns any application of fully $\mathrm{HE}$, is that, in its state today, fully $\mathrm{HE}$ is extremely computationally intensive as discussed in $\$ 2.2 .1 .2$. Further, even assuming performance similar to that of RSA, this solution would be hundreds of times slower than our GC-based solution, as symmetric primitives used in GC are orders of magnitude faster. Finally, from the leakage-resilience perspective, the UNMASK algorithm will be problematic, as it would need to perform complicated decryptions based on the secret key. We would need to ensure nothing is leaked in these modules, which would bring us either to using much stronger assumptions or to a chicken-andegg problem.

\subsubsection{Evaluating GCs in $\mathrm{HW}$}

In this section we describe how GCs (and hence also OTPs) can be efficiently evaluated on embedded systems and memory-constrained devices. First, we discuss how GCs can 
be evaluated with low memory (\$4.2.3.1). Then, we describe an architecture for evaluating GCs efficiently in $\mathrm{HW}(\$ 4.2 .3 .2)$ and present compile-time optimizations for that architecture (\$4.2.3.3). Finally, we give timing results on a prototype implementation (\$4.2.3.4.

Running Example: SFE of AES. We stress that our designs and optimizations are generic. However, for concreteness and for meaningful comparison of our $[\mathrm{HW}] \mathrm{im}-$ plementation with prior software implementations of SFE we choose secure evaluation of the AES functionality as example. SFE of AES was considered in [PSSW09] as a useful and representative function, with applications such as Oblivious Pseudo-Random Function (OPRF), blind Message Authentication Codes (MACs) and encryption, and computation on encrypted data. In this setting, sender $\mathcal{S}$ provides AES key $k$ as input $y$ and receiver $\mathcal{R}$ provides a plaintext block $m$ as input $x$. $\mathcal{R}$ obtains the ciphertext $c$ as output $z$, where $c=\operatorname{AES}(k, m)$. Recall, during GC evaluation (EVAL), both key and message are masked and hence cannot be leaked.

\subsubsection{GC Evaluation with Low Memory ${ }^{11}$}

For evaluating the $\mathrm{GC}, \mathcal{C}$ starts with the garbled values of the inputs. Then, $\mathcal{C}$ obtains the garbled gates one-by-one and evaluates them. The obtained garbled output for the gate needs to be stored by $\mathcal{C}$ until it is used the last time as input into a garbled gate.

To reduce the memory footprint to store intermediate values during $\mathrm{GC}$ evaluation, a "good" topologic order can be chosen. As pointed out in Ber91, the problem of finding a topologic order of a circuit that minimizes the needed amount of memory to store intermediate values is equivalent to the register allocation problem. This problem is well-studied in the context of compilers. In fact, algorithms for register allocation (e.g., $\mathrm{CAC}^{+} 81$, SS02]) can be used to find a good topologic order of the circuit which reduces the amount of memory needed for its evaluation.

In the worst case, the memory needed to evaluate a circuit $C$ is linear in the circuit size as many intermediate values need to be stored. Fig. 4.5 shows an (artificially constructed) example circuit consisting of $k$ gates which requires $\Theta(k)$ memory for evaluation.

In our $\mathrm{HW}$ architecture presented in the following, we use only three registers for caching and leave the investigation of larger cache sizes as future work.

\subsubsection{Architecture for Evaluating GCs in $\mathrm{HW}$}

We describe our architecture for efficient evaluation of GC on memory-constrained devices, i.e., having a small amount of slow memory only.

To minimize overhead, we choose key length $t=127$; with a permutation bit, garbled values are thus 128 bits long (cf. \$2.2.2). In the following we assume that memory

\footnotetext{
${ }^{11}$ This subsection first appeared in the full version of [JKSS10a] and was published in JKSS10b].
} 


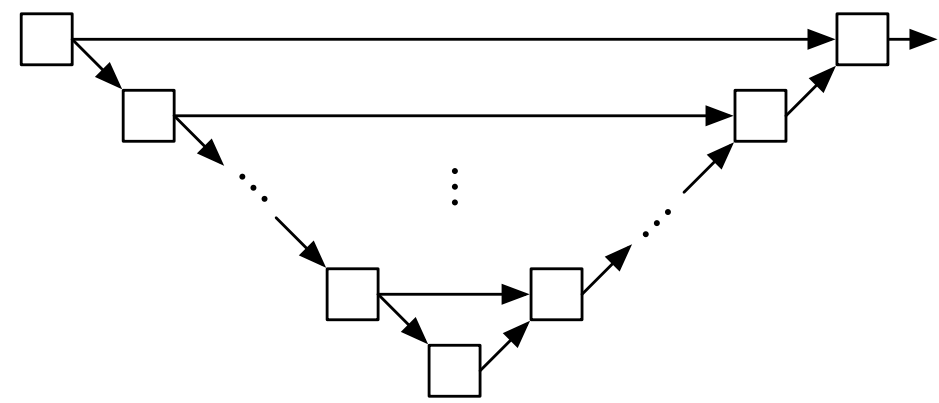

Figure 4.5: Circuit of $k$ Gates which requires $\Theta(k)$ Memory for Evaluation

cells and registers store 128 bit garbled values. This can be mapped to standard HW architectures by using multiple elements in parallel.

Fig. 4.6 shows a conceptual high-level overview of our architecture described next. At the high-level, EVAL, the process of evaluating GC, on our architecture consists of the following steps (cf. $\$ 4.2 .2 .3$ ). First, the garbled input values $\widetilde{x}, \widetilde{y}$ are stored in memory using the I/O interface. Then, GC gates are evaluated, using registers $\mathrm{A}, \mathrm{B}$, and $\mathrm{C}$ to cache the garbled inputs and outputs of a single garbled gate. Finally, the garbled output value $\widetilde{z}$ is output over the I/O interface.

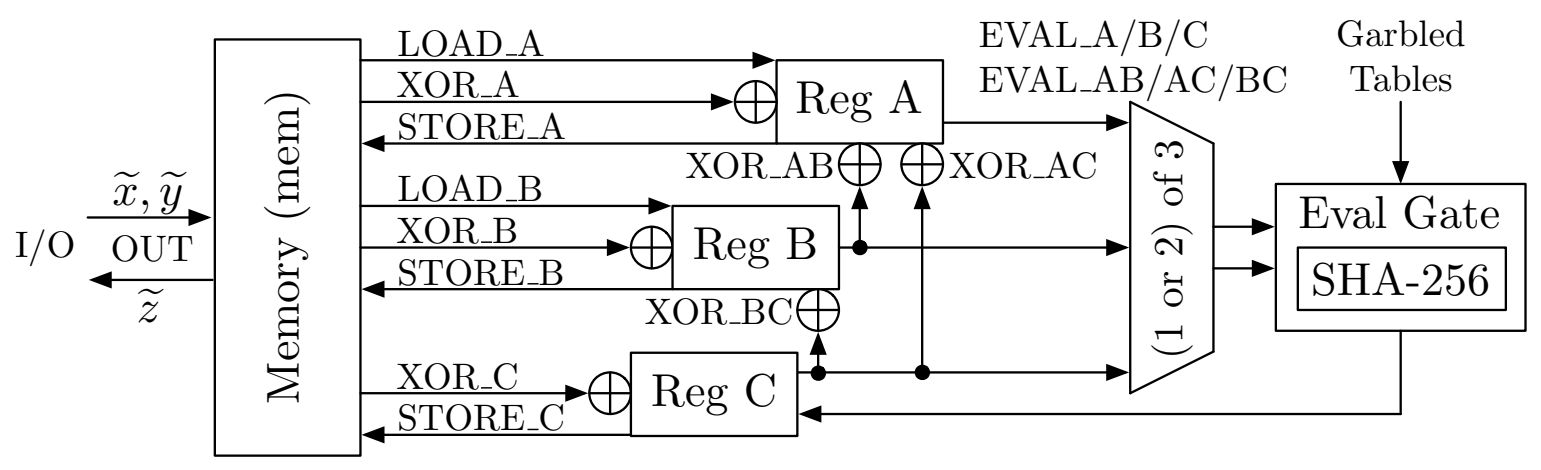

Figure 4.6: Architecture: GC Evaluation on Memory-Constrained Devices

As memory access is expensive (cf. $\$ 4.2 .3 .4$ ) we optimize the code to re-use values already in registers. Our instructions are one-address, i.e., each instruction consists of an operator and up to one memory address. Each of our instructions has length 32 bits: 5 bits to encode one of 18 instructions (described next) and 27 bits to encode an address in memory. 
LOAD/STORE: Registers can be loaded from memory using LOAD instructions LOAD_A and LOAD_B. Register C cannot be loaded as it will hold the output of evaluated non-XOR gates (see below). Values in registers can be stored back into memory using STORE instructions STORE_A, STORE_B, and STORE_C.

XOR: We evaluate XOR gates [KS08a as follows. XOR_A addr computes $A \leftarrow$ $A \oplus$ mem[addr]. Similarly, the other one-operand XOR operations (XOR1) XOR_B and XOR_C xor the value from memory with the value in the respective register. To compute XOR gates where both inputs are already in registers (XOR2), the instruction XOR_AB computes $A \leftarrow A \oplus B$. Similarly, XOR_AC computes $A \leftarrow A \oplus C$ and XOR_BC computes $B \leftarrow B \oplus C$.

EVAL: Non-XOR gates are evaluated with the Eval Gate block that contains a HW accelerator for SHA 256. As efficient GC technique we use Garbled Row Reduction of [NP01] as described in $\$ 2.2 .2 .3$. The garbled inputs are in one (EVAL1) or two registers (EVAL2), and the result is stored in register $\mathrm{C}$. The respective instructions for 1-input gates are EVAL_A, EVAL_B, EVAL_C and for 2-input gates EVAL_AB, EVAL_AC, EVAL_BC. The required garbled table entry for evaluating the garbled non-XOR gate is read from memory.

I/O: The garbled inputs are always stored at the first $|x|+|y|$ memory addresses. The garbled outputs are obtained from memory with OUT instructions.

Example 8. Fig. 4.7 shows an example circuit and a possible sequence of instructions to evaluate it on our architecture. First, register $A$ is loaded with $\widetilde{x}_{1}$ from memory address $0 x 0$, then $\widetilde{x}_{2} \oplus \widetilde{y}_{1}$ is computed in register $B$ and the AND gate is evaluated to obtain output $\widetilde{z}_{1}$ which is stored at address $0 x 0$ and overwrites $\widetilde{x}_{1}$, which is no longer needed. Then, the NOT gate is computed using register $B$ as input and stored at address $0 x 1$. The two outputs $\widetilde{z}_{1}, \widetilde{z}_{2}$ are at addresses $0 x 0$ and $0 x 1$.

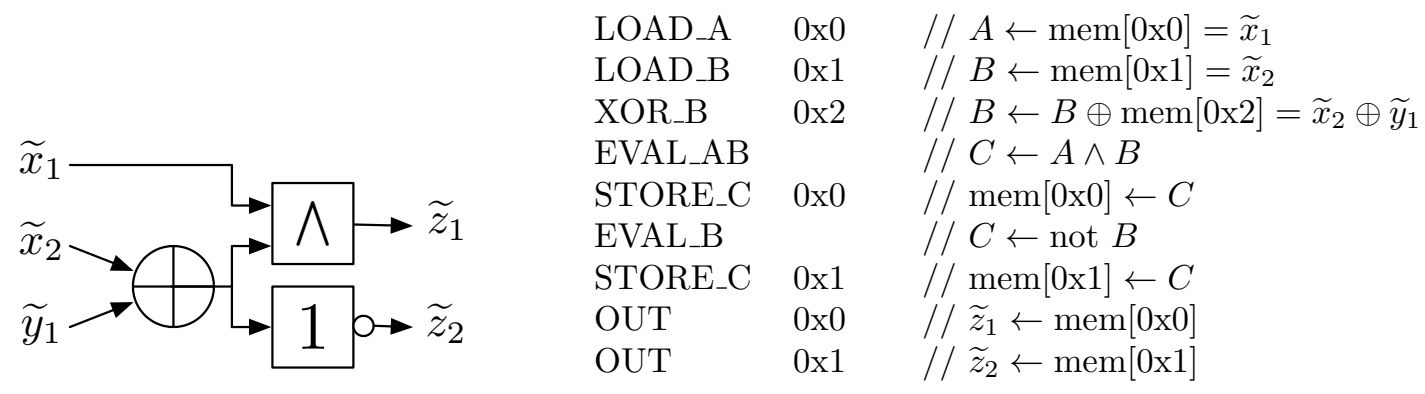

(a) Example Circuit

(b) Instruction Sequence

Figure 4.7: Example: Circuit and Instruction Sequence (Example 8) 


\subsubsection{Optimizations for Memory-Constrained Devices}

In this section, we present several compile-time optimizations to improve performance of $\mathrm{GC}$ evaluation (EVAL) on our $\mathrm{HW}$ architecture. We aim to reduce the size of GC (by minimizing the number of non-XOR gates), the size of the program (number of instructions), the number of memory accesses and memory size for storing intermediate garbled values. For concreteness, we use AES as representative functionality for the optimizations and performance measurements, but our techniques are generic.

Optimization a:Base. Our baseline is the AES circuit/code of [PSSW09], already optimized for a small number of non-XOR gates. Their circuit consists of 11, 286 twoinput non-XOR gates; thus, its $\mathrm{GC}$ has size $11,286 \cdot 3 \cdot 128$ bit $\approx 529$ kByte. Without considering any caching strategies, this results in 113,054 instructions, hence the program size is $113,054 \cdot 32$ bit $\approx 442 \mathrm{kByte}$, and the total amount of memory needed for EVAL is $34,136 \cdot 128 \mathrm{bit} \approx 533 \mathrm{kByte}$.

We start with further reduction of the size of the GC.

Optimization b:NoXNOR. First, we reduce the GC size by applying the optimization techniques described in \$3.2.1: We replace XNOR gates with XOR gates, and propagate the inverted output into the successor gates. Output XNOR gates are replaced with XOR and a 1-input inverter gate. The cost of this optimization is linear in the size of the circuit [PSS09]. Overall, this optimization results in the elimination of 4,086 XNOR gates and reduces the size of the AES GC to $(7,200 \cdot 3+40) \cdot 128 \mathrm{bit} \approx 338 \mathrm{kByte}($ improvement of $36 \%)$.

The remaining optimizations use b:NoXNOR.

Optimization c:Cache. We re-use values already in registers to reduce the number of LOADs. Values in registers are saved to memory only if needed later.

The remaining optimizations use c:Cache.

Optimization d:MaxFanout. We select a specific topologic order (traversing the circuit depth-first and following children in decreasing order of their fan-out).

Optimization e:Rand. We randomly consider several orders of evaluation, and select the most efficient one for EVAL. (This is a one-time compilation expense per function.) For present work, we considered several random topologic orders of the circuit, constructed by the traversal where the next gate is selected at random from the set of unprocessed gates with maximal fan-out. A more rigorous approach to this randomized technique can result in more substantial improvement, and is a possible direction for future work. 
Result. Using our optimizations we were able to substantially decrease the memory footprint of EVAL. As shown in Table 4.3, the smallest program was obtained with the non-deterministic optimization e:Rand. This is only slightly better than our best deterministic optimization d:MaxFanout and improves over a:Base as follows. The size of the AES program $P$ is only $73,583 \cdot 32$ bit $\approx 287$ kByte (improvement of $34.9 \%$ ). The amount of intermediate memory is $17,315 \cdot 128$ bit $\approx 271$ kByte (improvement of $49.3 \%$ ) and the number of memory accesses (read and write) is reduced by $\approx 35 \%$.

Table 4.3: AES Circuit Optimized for Low Memory (Sizes in kB)

\begin{tabular}{|c|c|c|c|c|c|c|c|c|}
\hline & \multicolumn{2}{|c|}{$\mathrm{GC} \widetilde{C}$} & \multicolumn{2}{|c|}{ Program $P$} & \multicolumn{4}{|c|}{ Memory for $\mathrm{GC}$ Evaluation } \\
\hline Optir & $n-\mathrm{XOR}$ & Size & Instr. & Size & Read & Write & Entries & Size \\
\hline$\overline{\mathrm{a}: \mathrm{Bas}}$ & L,286 & 529 & 13,054 & 442 & 67,760 & 33,880 & 34,136 & 533 \\
\hline & 7,240 & 338 & 10 & 426 & 0 & 20 & 34 & 34 \\
\hline & 7,240 & 338 & 95 , & 375 & 56,779 & 30 , & 21 & 332 \\
\hline & 7,240 & 338 & 74 & 289 & 42,469 & 23 & 18, & 292 \\
\hline e:Rand & 7,240 & 338 & 73,583 & 287 & 42,853 & 22,650 & 17,315 & 271 \\
\hline
\end{tabular}

\subsubsection{Hardware Implementation}

We have mapped the $\mathrm{HW}$ architecture for evaluating $\mathrm{GCS}$ of $\$ 4.2 .3 .2$ to two common system architectures as shown in Fig. 4.8, one for a System On a Programmable Chip (SOPC) with a HW accelerator for a cryptographic hash function (reflecting smartcard and future smartphone architectures) and another for a stand-alone unit (reflecting a custom-made $\mathrm{HW}$ accelerator for GC evaluation). In both architectures, the inputs (program $P, \mathrm{GC} \widetilde{C}$, and garbled inputs $\widetilde{x}, \widetilde{y}$ ) and the garbled outputs $\widetilde{z}$ are transferred between the host and the RAM of our $\mathrm{HW}$ accelerator over the I/O interface: in the beginning, the host writes the inputs to the RAM and, in the end, the outputs are written into specific addresses from which the host retrieves them.

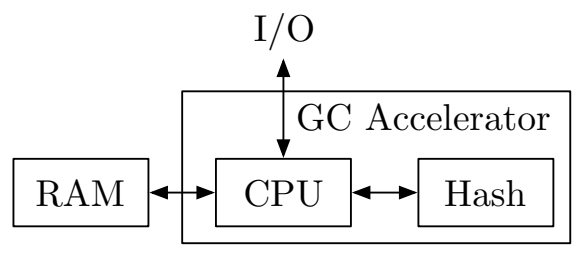

(a) System On a Programmable Chip

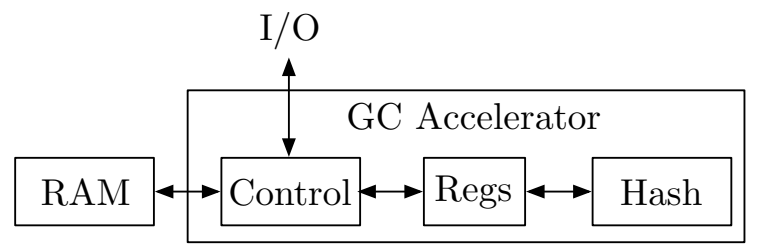

(b) Stand-Alone Unit

Figure 4.8: System Architectures: Hardware-Assisted GC Evaluation 
SOPC The SOPC architecture shown in Fig. 4.8(a) consists of a processor (CPU) with access to RAM and a $\mathrm{HW}$ accelerator for a cryptographic hash function (e.g., SHA -256) to speed up the most computational burden of the GC evaluation. This is a representative architecture for next generation smartphones or smartcards such as the STMicroelectronics ST33F1M smartcard which includes a 32-bit RISC processor, cryptographic peripherals, and memory comparable to our prototype system [STM08].

Stand-Alone Unit. The stand-alone architecture shown in Fig. 4.8(b) consists of a custom-made control state machine, registers (A, B, C), a Hash unit (e.g., SHA256), and RAM. This architecture could be used to design an Application Specific IC (ASIC) or a FPGA as high-speed $\mathrm{HW}$ accelerator for $\mathrm{GC}$ evaluation. When the host has written the inputs to the RAM, the stand-alone unit executes the program. The state machine parses the program and moves data between RAM and registers or evaluates the non-XOR gates using the Hash unit according to the instructions (cf. $\$ 4.2 .3 .2$ for details).

Performance evaluation. Both system architectures (using SHA-256 as cryptographic hash function) have been implemented and evaluated on an Altera/Terasic DE1 FPGA board comprising an Altera Cyclone II EP2C20F484C7 FPGA and $512 \mathrm{kB}$ SRAM and $8 \mathrm{MB}$ SDRAM running at $50 \mathrm{MHz}$. For details on the architecture and area requirements we refer to [JKSS10c] and concentrate on the timings in the following.

Table 4.4: Timings: Instructions on Prototypes (clock cycles, average)

\begin{tabular}{|l||r|r|}
\hline Instruction & SOPC & Stand-Alone Unit \\
\hline \hline LOAD & 291.43 & 87.63 \\
\hline XOR1 & 395.30 & 87.65 \\
XOR2 & 252.00 & 1.00 \\
\hline STORE & 242.00 & 27.15 \\
\hline EVAL1 & $1,282.30$ & 109.95 \\
EVAL2 & $1,491.68$ & 135.05 \\
\hline OUT & 581.48 & 135.09 \\
\hline
\end{tabular}

Instruction Timings. The timings of instructions are summarized in Table 4.4. They show the average number of clock cycles required to execute an instruction excluding the latency of fetching the instruction. Gate evaluations are expensive in the SOPC implementation, although the SHA-256 computations are fast, because they involve a lot of data movement (to/from the Hash unit and from the RAM) which is expensive. The dominating role of memory reads and writes is clear in the timings of the standalone implementation: the only instructions that do not require memory operations (XOR2) require only a single clock cycle. Due to the Garbled Row Reduction GC technique (cf. 22.2 .2 .3 which eliminates the first garbled table entry, EVAL1 is faster 
than EVAL2 because it accesses the memory on average every other time compared to three times out of four.

Table 4.5: Timings: GC Evaluation of AES on Prototypes

\begin{tabular}{|l||r|r||r|r|}
\hline \multicolumn{1}{|c||}{} & \multicolumn{2}{c||}{ SOPC } & \multicolumn{2}{c|}{ Stand-Alone Unit } \\
\hline \multicolumn{1}{|c||}{ Optimization } & Timings (ms) & \multicolumn{2}{c|}{ Timings (ms) } \\
& SHA & Total & SHA & Total \\
\hline \hline a:Base & 15 & 1,894 & 15 & 225 \\
\hline b:NoXNOR & 10 & 1,749 & 10 & 212 \\
c:Cache & 10 & 1,560 & 10 & 184 \\
d:MaxFanout & 10 & 1,259 & 10 & 144 \\
e:Rand & 10 & 1,253 & 10 & 144 \\
\hline
\end{tabular}

AES Timings. The timings to evaluate the optimized GCs for the AES functionality of $\$ 4.2 .3 .3$ on the prototype implementations are given in Table 4.5. These timings are for $\mathrm{GC}$ evaluation only; i.e, they neglect the time for transferring data to/from the system because interface timings are highly technology dependent. The SHA-256 computations take an equal amount of time for both implementations as the Hash unit is the same. The (major) difference in timings is caused by data movement, XORs, interface to the Hash unit, etc. The runtimes for both implementations are dominated by writing and reading the SDRAM; e.g., $84.3 \%$ for the stand-alone unit and our smallest AES circuit (optimization e:Rand). Hence, accelerating memory access, e.g., with burst reads and writes, is the key for further speedups.

Performance Comparison. A software implementation that securely evaluates the GCDTP of the unoptimized AES functionality a:Base required 2 seconds on an Intel Core 2 Duo 3.0 GHz with $4 \mathrm{~GB}$ RAM [PSSW09]. Our optimized circuit e:Rand evaluated on the stand-alone unit requires only $144 \mathrm{~ms}$ for the same operation and, therefore, provides a speedup by a factor of 10.4-17.4 (taking the lack of precision into account).

Clearly, GC/OTP evaluation is substantially slower than evaluation of the plain functionality in $\mathrm{HW}$ (a straight-forward iterative implementation of AES-128 takes only 10 clock cycles to encrypt one message block). Also the timing overhead when protecting against specific attacks only is substantially smaller (e.g., factor 3.88 for protection against differential power analysis $\left.\left[\mathrm{THH}^{+} 05\right]\right)$.

Still, using provably leakage-resistant evaluation based on GC OTP might be justified for applications which require high security guarantees and/or are not evaluated too frequently, 


\subsection{Application: Privacy-Preserving Cloud Computing}

In this section we show how the token for creating Garbled Circuits (GCs) of $\$ 4.1 .4$ can be extended and used for secure outsourcing of data and arbitrary computations thereon in a cloud computing scenario.

Secure outsourcing of computation to an untrusted (cloud) service provider is becoming more and more important. Pure cryptographic solutions based on fully homomorphic and verifiable encryption, recently proposed, are promising but suffer from very high latency. Other proposals perform the whole computation on tamper-proof hardware (HW) and usually suffer from the same problem.

In the following we focus on applications where the latency of the computation should be minimized, i.e., the time from submitting the query until receiving the result should be as small as possible. To achieve this, we show how to combine a trusted HW] token (e.g., a cryptographic coprocessor or provided by the customer) with Secure Function Evaluation (SFE) to compute arbitrary functions on secret (encrypted) data where the computation leaks no information and is verifiable. The token is used in the setup phase only whereas in the time-critical online phase the cloud computes the encrypted function on encrypted data using symmetric encryption primitives only and without any interaction with other entities.

Publication Info: Parts of the following results are published in [SSW10].

\subsubsection{Motivation}

Enterprises and other organizations often have to store and operate on a huge amount of data. Cloud computing offers infrastructure and computational services on demand for various customers on shared resources. Services that are offered range from infrastructure services such as Amazon EC2 (computation) [AmEa] or S3 (storage) [AmS], over platform services such as Google App Engine [GoA] or Microsoft's database service SQL Azure [MiA], to software (SW) services such as outsourced customer relationship management applications by Salesforce.com.

While sharing IT infrastructure in cloud computing is cost-efficient and provides more flexibility for the clients, it introduces security risks organizations have to deal with in order to isolate their data from other cloud clients and to fulfill confidentiality and integrity demands. Moreover, since the IT infrastructure is now under control of the cloud provider, the customer has not only to trust the security mechanisms and configuration of the cloud provider, but also the cloud provider itself. When data and computation is outsourced to the cloud, prominent security risks are: malicious code that is running on the cloud infrastructure could manipulate computation and force wrong results or steal data; personnel of the cloud provider could misuse their 
capabilities and leak data; and vulnerabilities in the shared resources could lead to data leakage or manipulated computation [Clo10]. In general, important requirements of cloud clients are that their data is processed in a confidential way (confidentiality), and that their data and computation was processed in the expected way and has not been tampered with (integrity and verifiability).

Verifiable Computing. Secure outsourcing of arbitrary computation and data storage is particularly difficult to fulfill if a cloud client does not trust the cloud provider at all. There are proposals for cryptographic methods which allow to perform specific computations on encrypted data APRS01, or to securely and verifiably outsource storage [KL10]. Arbitrary computation on confidential data can be achieved by means of verifiable computing. The approach of GGP10 combines fully Homomorphic Encryption (HE) for confidentiality (cf. \$2.2.1.2) with Garbled Circuits (GCs) (cf. \$2.2.2) for integrity and verifiability (cf. \$2.3.2). The improved scheme of [CKV10] avoids GCs, but is based on fully $\mathrm{HE}$ as well. While these cryptographic schemes can fulfill the aforementioned requirements, they are currently not usable in practice due to the low efficiency of fully $\mathrm{HE}$ (cf. \$2.2.1.2).

Trusted Computing. Another line of works tries to solve these problems by establishing trusted execution environments where the cloud client can verify the integrity of the $\mathrm{SW}$ and the configuration of the cloud provider's $\mathrm{HW}$ platform. This requires, however, secure SW] such as secure hypervisors for policy enforcement and attestation mechanisms for integrity verification. The use of trusted computing-based remote attestation in the cloud scenario was recently discussed in [CGJ $\left.{ }^{+} 09\right]$. Trusted Virtual Domains $\left[\mathrm{BGJ}^{+} 05, \mathrm{CDE}^{+} 10\right]$ are one approach that combines trusted computing, secure hypervisors, and policy enforcement of information flow within and between domains of virtual machines. However, those approaches require trust in a non-negligible amount of HW] (e.g., CPU and Trusted Platform Module (TPM) [TCGT09]) which are under the physical control of the cloud provider. According to the specification of the Trusted Computing Group, the TPM is not designed to protect against HW attacks, but provides a shielded location to protect keys. However, the TPM cannot perform arbitrary secure computations on data. It can protect cryptographic keys and perform only pre-defined cryptographic operations like encryption, decryption, and signature creation. In particular, if data should be encrypted it must be provided in plaintext to the TPM, and if data should be decrypted it will be given in plaintext as output. Unfortunately, the TPM cannot be instructed to decrypt data internally, perform computations on the data, and encrypt it again before returning the output. A virtualized TPM $\left[\mathrm{BCG}^{+} 06\right]$ that is executed in $\mathrm{SW}$ could be enhanced with additional functionality (see, e.g., SSW08]). However, such SW running on the CPU has access to unencrypted data at some point to compute on it. Hence, if the cloud provider is malicious and uses specifically manipulated $\mathrm{HW}$, confidentiality and 
verifiability cannot be guaranteed by using trusted computing.

Tamper-Proof Hardware. A HW token which is tamper-proof against physical attacks but can perform arbitrary computations would enable the cloud client to perform confidential and verifiable computation on the cloud provider's site, given that the client trusts the manufacturer of the token that it does not leak any information to the provider. For example, secure coprocessors [SW99, Yee94] are tamper-proof active programmable devices that are attached to an untrusted computer in order to perform security-critical operations or to allow to establish a trusted channel through untrusted networks and $\mathrm{HW}$ devices to a trusted $\mathrm{SW}$ program running inside the secure coprocessor. This can be used to protect sensitive computation from insider attacks at the cloud provider [JSM01]. If cloud providers offer such tokens produced by trustworthy third-party manufacturers, or offer interfaces to attach $\mathrm{HW}$ tokens provided by clients to their infrastructure (and by assuming $\mathrm{HW}$ is really tamper-proof), cloud clients could perform their sensitive computations inside those tokens. Data can be stored encrypted outside the token in cloud storage while decryption keys are stored in shielded locations of the trusted tokens.

The token based approach is reasonable because both, cryptographic coprocessors and standardized interfaces (e.g., smartcard readers or PCI extension boards) exist that can be used for such tokens. Of course, for trust reasons, the token vendor should not be the same as the cloud provider. However, the whole security-critical computation takes place in the token. Hence, such computation is not really outsourced to the cloud because the function is computed within the token. Some applications, however, require fast replies to queries which cannot be computed online within the tamperproof token. For example, queries in personal health records or payroll databases may occur not very frequently, but need to be processed very fast while privacy of the data should be preserved.

In the following, we focus on cloud application scenarios where private queries to the outsourced data have to be processed and answered with low latency.

Outline and Contribution. The remainder of this section is structured as follows: First, we introduce our model for secure verifiable outsourcing of data and arbitrary computations thereon in $\$ 4.3 .2$. In $\$ 4.3 .3$ we present architectures to instantiate our model: The first architecture computes the function within a tamper-proof HW token (\$4.3.3.1) and the second architecture is based on fully $\mathrm{HE}(\$ 4.3 .3 .2)$.

Our main technical contribution is a third architecture (\$4.3.3.3) that combines the advantages of the previous architectures and overcomes their disadvantages. Our solution uses a resource constrained tamper-proof $\mathrm{HW}$ token $\mathcal{T}$ in the setup pre-processing phase. Then, in the online phase only symmetric cryptographic operations are performed in parallel within the cloud without further interaction with $\mathcal{T}$. For this, we adopt the embedded SFE protocol of $\$ 4.1$ to the large-scale cloud-computing scenario. 
Finally, in $\$ 4.3 .4$ we compare the performance of all three proposed architectures and show that our scheme allows secure verifiable outsourcing of data and arbitrary computations thereon with low latency.

\subsubsection{Model for Privacy-Preserving Cloud Computing}

We consider the model shown in Fig. 4.9 that allows a client $\mathcal{C}$ to verifiably and securely outsource a database $D$ and computations thereon to an untrusted (cloud) service provider $\mathcal{S}$.

A client $\mathcal{C}$ (e.g., a company) wants to securely outsource data $D$ and computation of a function $f$ (represented as a boolean circuit) thereon to an untrusted service provider $\mathcal{S}$ who offers access to (cloud) storage services and to (cloud) computation services. Example applications include outsourcing of medical data, log files or payrolls and computing arbitrary statistics or searches on the outsourced data. In addition, the evaluation of $f$ can depend on a session-specific private query $x_{i}$ of $\mathcal{C}$ resulting in the response $y_{i}=f\left(x_{i}, D\right)$. However, $\mathcal{S}$ should be prevented from learning or modifying $D$ or $x_{i}$ (confidentiality and integrity) or to compute $f$ incorrectly (verifiability) ${ }^{12}$ Any cheating attempts of a malicious $\mathcal{S}$ who tries to deviate from the protocol should be detected by $\mathcal{C}$ with overwhelming probability where $\mathcal{C}$ outputs the special failure symbol $\perp 13$

While this scenario can easily be solved for a restricted class of functions (e.g., private search of a keyword $x_{i}$ using searchable encryption [KL10]), we consider the general case of arbitrary functions $f$. Due to the large size of $D$ (e.g., a database) and/or the computational complexity of $f$, it is not possible to securely outsource $D$ to $\mathcal{S}$ only and let $\mathcal{C}$ compute $f$ after retrieving $D$ from $\mathcal{S}$. Instead, the confidentiality and integrity of the outsourced data $D$ has to be protected while at the same time secure computations on $D$ need to be performed at $\mathcal{S}$ without interaction with $\mathcal{C}$.

\subsubsection{Tamper-Proof Hardware Token $\mathcal{T}$}

To improve the efficiency of the secure computation, our model additionally allows that $\mathcal{C}$ uses a tamper-proof $H W$ token $\mathcal{T}$, integrated into the infrastructure of $\mathcal{S}$, that is capable of performing computations on behalf of $\mathcal{C}$ within a shielded environment, i.e., $\mathcal{T}$ must be guaranteed not to leak any information to $\mathcal{S}$. As $\mathcal{T}$ needs to be built tamper-proof and cost-effective, it will have a restricted amount of memory only. In many cases the available memory within $\mathcal{T}$ will not be sufficient to store $D$ or intermediate values during evaluation of $f$. If needed, $\mathcal{T}$ might resort to additional (slow) secure external memory (e.g., GKM09]).

\footnotetext{
${ }^{12} \mathcal{S}$ might attempt to cheat to save storage/computing resources or manipulate the result.

${ }^{13}$ As detailed in GGP10 it must be guaranteed that $\mathcal{S}$ cannot learn whether $\mathcal{C}$ detected an error or not to avoid that $\mathcal{S}$ can use this single bit of information to construct a decryption or verification oracle.
} 


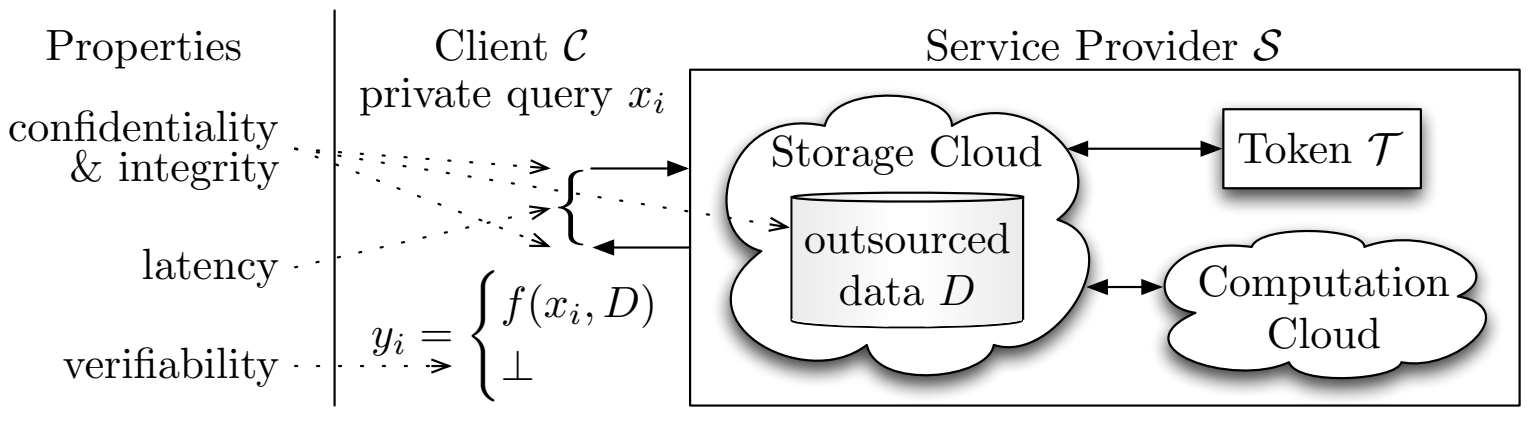

Figure 4.9: Model: Secure Outsourcing of Data and Arbitrary Computations

The token $\mathcal{T}$ could be instantiated with a cryptographic coprocessor built by a thirdparty manufacturer whom $\mathcal{C}$ trusts in a way that $\mathcal{T}$ does not leak any information to $\mathcal{S}$. A possible candidate would be the IBM Cryptographic Coprocessor 4758 or its successor 4764 which is certified under FIPS PUB 140-2 [SW99, IBM]. Such cryptographic coprocessors allow to generate secret keys internally and securely transport them to $\mathcal{C}$ or to another token for migration purposes, and authentication to verify that the intended $[\mathrm{SW}]$ is executed within the shielded environment. (For details on migrating a state (key) between two trusted environments (cryptographic coprocessors) we refer to [BCG ${ }^{+}$66, SSW08].) As such tokens based on cryptographic coprocessors can be used for multiple users in parallel, their costs amortize for service provider and users.

For extremely security critical applications where $\mathcal{C}$ does not want to trust the manufacturer of cryptographic coprocessors offered by $\mathcal{S}, \mathcal{C}$ can choose her own $\mathrm{HW}$ manufacturer to produce the tamper-proof HW] token $\mathcal{T}$ and ship this to $\mathcal{S}$ for integration into his infrastructure. We note that this approach is similar to "server hosting" which assumes outsourcing during long periods. However, this contradicts the highly dynamic cloud computing paradigm where service providers can be changed easily.

\subsubsection{Preliminaries and Notation}

In the following we make use of fully $\mathrm{HE}$ and $\mathrm{GCs}$ as defined in $\$ 2$.

Notation. $\llbracket x \rrbracket$ denotes the fully $\mathrm{HE}$ of a value $x$, and $\widetilde{x}$ the garbled value corresponding to $x$.

Encryption and Authentication. Confidentiality and authenticity of messages can be guaranteed either symmetrically (using one key) or asymmetrically (using two keys).

The symmetric case can be instantiated with a combination of symmetric encryption (e.g., AES [NIS01]) and a Message Authentication Code (MAC) (cf. 2.1.2). These 
schemes use a respective symmetric key for encryption/authentication and the same key for decryption/verification.

Alternatively, public-key cryptography (e.g., RSA or EC-based ElGamal/DSA) allows usage of separate keys for encryption/authentication and other keys for decryption/verification. This could be used for example to construct an outsourced database to which new entries can be appended by multiple parties without using shared symmetric keys (cf. Fig. 4.9).

Notation. $\widehat{x}$ denotes authenticated and $\bar{x}$ encrypted and authenticated data $x$.

\subsubsection{Architectures for Privacy-Preserving Cloud Computing}

In the following we present three architectures for instantiating our model of $\$ 4.3 .2$.

\subsubsection{Token Computes}

A first approach, also used in [JSM01], is to let the token $\mathcal{T}$ compute $f$ as shown in Fig. 4.10. For this, $\mathcal{C}$ and $\mathcal{T}$ share symmetric keys for encryption and verification. The encrypted and authenticated database $\bar{D}$ and the authenticated function $\widehat{f}$ is stored within the storage cloud of service provider $\mathcal{S}$. In the online phase, $\mathcal{C}$ sends the encrypted and authenticated query $\bar{x}_{i}$ to $\mathcal{T}$ and the storage cloud provides $\bar{D}$ and $\widehat{f}$ one-by-one. $\mathcal{T}$ decrypts and verifies these inputs and evaluates $y_{i}=f\left(x_{i}, D\right)$ using secure external memory to store intermediate values ${ }^{114}$ If $\mathcal{T}$ detects any inconsistencies, it continues evaluation substituting the inconsistent value with a random value, and sets $y_{i}$ to the failure symbol $\perp$. Finally, $\mathcal{T}$ sends the authenticated and encrypted response $\bar{y}_{i}$ back to $\mathcal{C}$ who verifies and decrypts $\bar{y}_{i}$ to obtain the output $y_{i}$.

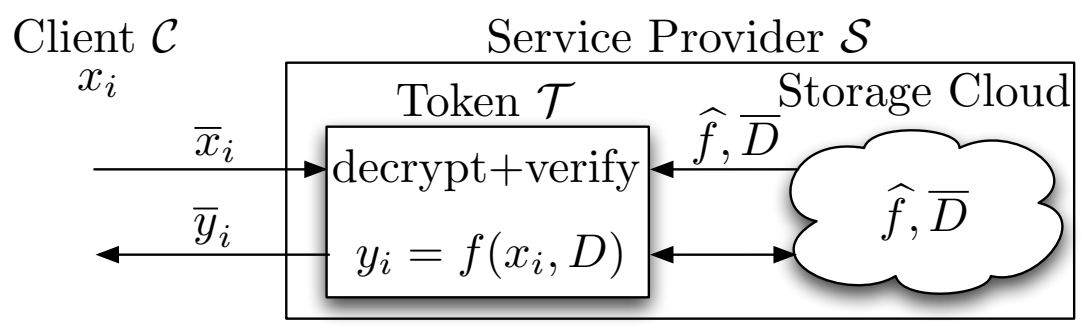

Figure 4.10: Architecture: Token Computes [JSM01]

Performance. In this approach, the latency of the online phase, i.e., the time from sending the query $x_{i}$ to receiving the response $y_{i}$, depends on the performance of $\mathcal{T}$ (in particular on the performance of secure external memory) and cannot be improved by using the computation cloud services offered by $\mathcal{S}$.

\footnotetext{
${ }^{14}$ In the worst case, the amount of required external memory can be up to linear in the size of the function as shown in 4.2 .3 .1 .
} 


\subsubsection{Cloud Computes}

The approach of GGP10 shown in Fig. 4.11 does not require a trusted HW token but combines GCs for verifiability (cf. UNMASK step in $\$ 4.2 .2 .3$ ) and integrity with fully $\mathrm{HE}$ for confidentiality of the outsourced data and computations. The main idea is to evaluate the same $\mathrm{GC} \widetilde{f}$ under fully $\mathrm{HE}$ and use the resulting homomorphically encrypted garbled output values to verify that the computation was performed correctly:

Setup Phase. During setup, $\mathcal{C}$ generates a $\mathrm{GC} \tilde{f}$ and sends it to $\mathcal{S}$ 's storage cloud 15 To outsource the database $D$, the corresponding garbled values $\widetilde{D}$ are encrypted with the fully $\mathrm{HE}$ scheme and $\llbracket \widetilde{D} \rrbracket$ is stored in $\mathcal{S}$ 's storage cloud as well.

Online Phase. In the online phase, $\mathcal{C}$ sends the homomorphically encrypted garbled query $\llbracket \widetilde{x}_{i} \rrbracket$ to $\mathcal{S}$ who evaluates the $\mathrm{GC} \widetilde{f}$ on $\llbracket \widetilde{x}_{i} \rrbracket$ and $\llbracket \widetilde{D} \rrbracket$ using the homomorphic properties of the fully $\mathrm{HE}$ scheme. As result, $\mathcal{S}$ obtains $\llbracket \widetilde{y}_{i} \rrbracket=\llbracket \widetilde{f}\left(\widetilde{x}_{i}, \widetilde{D}\right) \rrbracket$ and sends this back to $\mathcal{C}$. After decryption, $\mathcal{C}$ obtains $\widetilde{y}_{i}$ and can verify whether the computation was performed correctly. Otherwise, $\mathcal{C}$ outputs the failure symbol $\perp$.

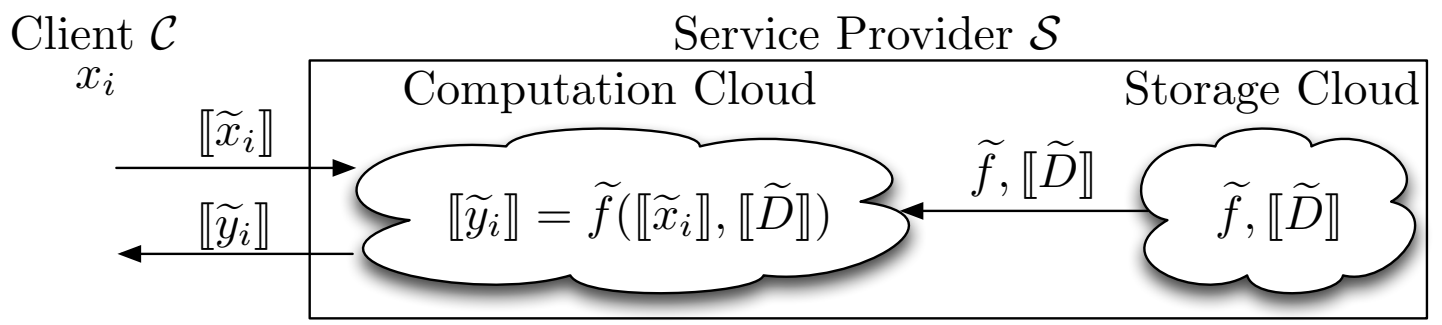

Figure 4.11: Architecture: Cloud Computes [GGP10]

Improvements. Several improvements have been proposed in CKV10] to avoid $\mathrm{GCs}$ and hence reduce the communication complexity in the setup phase. Still, these constructions require fully $\mathrm{HE}$.

Performance. The advantage of these approaches is that they do not require any trusted $\mathrm{HW}$ and hence can be computed in parallel in the computation cloud. However, due to the low performance of today's fully $\mathrm{HE}$ schemes, these approaches are unlikely to be used in practical applications in the near future (cf. \$2.2.1.2).

Impossibility Results. Indeed, as shown in [DJ10], the setting with a single client is the only one that allows for privacy-preserving cloud computing based on cryptography alone and hence $\mathrm{SW}$ only. When data is shared among more than one client, users

\footnotetext{
${ }^{15}$ This is a correction to the wrong notation and description in [SSW10, Sect. 4.2].
} 
of cloud services will also need to rely on other forms of privacy enforcement, such as tamper-proof HW, distributed computing, and complex trust ecosystems.

\subsubsection{Token Sets Up and Cloud Computes}

Our approach combines a tamper-proof HW token $\mathcal{T}$ used in the setup phase only with efficient computations performed in parallel in the computation cloud as shown in Fig. 4.12. The main idea is that $\mathcal{T}$ generates a GC during the setup phase and in the time-critical online phase the $\mathrm{GC}$ is evaluated in parallel by the computation cloud.

In detail, our architecture consists of the following three phases:

System Initialization. During System Initialization, the client $\mathcal{C}$ and the token $\mathcal{T}$ agree on a symmetric (long-term) key $k$ (cf. \$4.3.2.1). Additionally, $\mathcal{C}$ provides $\mathcal{S}$ with the authenticated function $\widehat{f}$ (represented as boolean circuit) and the authenticated and encrypted data $\bar{D}$ who stores them in the storage cloud.

Setup Phase. In the Setup Phase, $\mathcal{T}$ generates for protocol invocation $i$ an internal session key $k_{i}$ derived pseudo-randomly from the key $k$ and $i$. Using $k_{i}$ as seed for randomness generation, $\mathcal{T}$ generates a $\mathrm{GC} \widetilde{f}_{i}$ from the function $\widehat{f}$ and a corresponding garbled re-encryption $\widetilde{D}_{i}$ of the database $\bar{D}$ which are stored in the storage cloud: As described in $\$ 4.1 .4 .3$, the $\mathrm{GC}$ can be generated gate-by-gate using a constant amount of memory only. For each gate of $\widehat{f}, \mathcal{S}$ provides $\mathcal{T}$ with the description of the gate. $\mathcal{T}$ uses the session key $k_{i}$ to derive the gate's garbled input values and the garbled output value and returns the corresponding garbled table to $\mathcal{S}$. In parallel, $\mathcal{T}$ accumulates a hash of the gates requested for so far (e.g., by successively updating $h_{i}=H\left(h_{i-1} \| G_{i}\right)$ where $H$ is a cryptographic hash function and $G_{i}$ is the description of the $i$-th gate) which is finally used to verify authenticity of $\widehat{f}$ (cf. $\$ 4.1 .4 .1$ for details). Similarly, $\mathcal{T}$ can convert the authenticated and encrypted database $\bar{D}$ into its garbled equivalent $\widetilde{D}_{i}$ using constant memory only: For each element $\bar{d}$ in $\bar{D}, \mathcal{T}$ verifies and decrypts $\bar{d}$ and uses the session key $k_{i}$ to derive the corresponding garbled value $\widetilde{d}_{i}$ of $\widetilde{D}_{i}$. Finally, $\mathcal{T}$ provides $\mathcal{S}$ with an encrypted and authenticated OK message $\overline{\mathrm{OK}}_{i}$ that contains the session id and whether the verification of $\widehat{f}$ and all elements in $\bar{D}$ were successful $\left(\mathrm{OK}_{i}=\langle i, \top\rangle\right)$ or $\operatorname{not}\left(\mathrm{OK}_{i}=\langle i, \perp\rangle\right)$.

Online Phase. In the Online Phase, $\mathcal{C}$ derives the session key $k_{i}$ and uses this to create the garbled query $\widetilde{x}_{i}$ which is sent to $\mathcal{S}$. Now, the computation cloud evaluates the pre-computed GC $\widetilde{f}_{i}$ in parallel using the garbled query and the pre-computed garbled data $\widetilde{D}_{i}$ as inputs. The resulting garbled output $\widetilde{y}_{i}$ is sent back to $\mathcal{C}$ together with the $\mathrm{OK}$ message $\overline{\mathrm{OK}}_{i}$. Finally, $\mathcal{C}$ verifies that both phases have been performed 
correctly, i.e., $\overline{\mathrm{OK}}_{i}$ for the setup phase $\left(\mathrm{OK}_{i}=\langle i, \top\rangle\right)$ and valid garbled output keys $\widetilde{y}_{i}$ for the online phase.

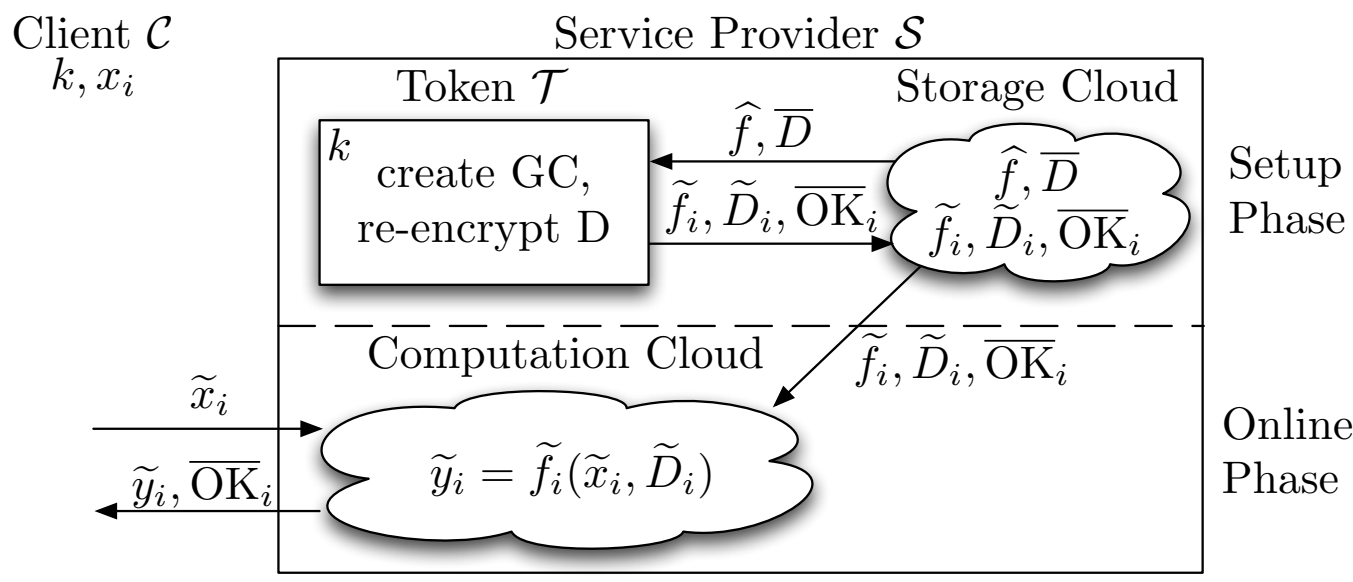

Figure 4.12: Our Architecture: Token Sets Up and Cloud Computes

Performance. Our entire architecture is based solely on symmetric cryptographic primitives and hence is very efficient. When $\mathcal{T}$ has access to a $\mathrm{HW}$ accelerator for GC creation (i.e., $\mathrm{HW}$ accelerators for Advanced Encryption Standard (AES) and SHA256 as described in \$4.1), the performance of the setup phase depends mostly on the speed of the interface between the token $\mathcal{T}$ and the storage cloud [JKSS10a]. The size of $\widetilde{f}_{i}$ and $\widetilde{D}_{i}$ is approximately $t$ times larger than the size of $\widehat{f}$ and $\bar{D}$, where $t$ is the symmetric security parameter (cf. $\$ 2.1 .1 .2$ ). To evaluate the $\mathrm{GC}$ in the online phase, one invocation of SHA-256 is needed for each non-XOR gate while XOR gates are "free" as described in $\$ 2.2 .2 .3$. GC evaluation can easily be parallelized for many practical functions that usually perform the same operations independently on every entry of the database, e.g., computing statistics or complex search queries.

Extensions. Our architecture can be naturally extended in several ways: To further speed up the setup phase, a trusted cloud consisting of multiple tokens can be used, that in parallel creates GCs and re-encrypts the database for multiple sessions of the same or different users, or even the same session. The function and the database can be updated dynamically when an additional monotonic revision number is used. Such updates can even be performed by multiple clients $\mathcal{C}_{i}$ by using public key encryption and signatures as described in $\$ 4.3 .2 .2$.

\subsubsection{Performance Comparison}

We conclude this section with a qualitative performance comparison of the proposed architectures and leave a prototype implementation for their quantitative performance 
comparison as future work.

As summarized in Table 4.6, the asymptotic complexity of the presented architectures is the same: the client $\mathcal{C}$ performs work linear in the size of the inputs $x_{i}$ and the outputs $y_{i}$, the storage cloud stores data linear in the size of the evaluated function $f$ and the outsourced data $D$ and the computation performed by the token $\mathcal{T}$ respectively the computation cloud is linear in the size of $f$. Hence, all three schemes are equally efficient from a complexity-theoretical point of view.

However, the online latency, i.e., the time between $\mathcal{C}$ submitting the encrypted query $x_{i}$ to the service provider $\mathcal{S}$ until obtaining the result $y_{i}$ differs substantially in practice.

For the token-based architecture of $\$ 4.3 .3 .1$, the online latency depends on the performance of the token $\mathcal{T}$ that evaluates $f$ and hence is hard to parallelize and might become a bottleneck in particular when $f$ is large and $\mathcal{T}$ must resort to secure external memory in the storage cloud.

The HE-based architecture of $\$ 4.3 .3 .2$ does not use a token and hence can exploit the parallelism offered by the computation cloud. However, this architecture is not ready for deployment in practical applications yet, as fully $\mathrm{HE}$ schemes are not yet sufficiently fast enough for evaluating a large functionality such as a GC under fully $\mathrm{HE}$.

Our proposed architecture of 4.3 .3 .3 achieves low online latency by combining both approaches: $\mathcal{T}$ is used in the setup phase only to generate a $\mathrm{GC}$ and to re-encrypt the database. In the online phase, the $\mathrm{GC} \widetilde{f}$ is evaluated in parallel by the computation cloud.

Table 4.6: Complexity: Architectures for Privacy-Preserving Cloud Computing

\begin{tabular}{|c|c|c|c|}
\hline Architecture & $\begin{array}{c}\mathcal{T} \text { Computes } \\
(\$ 4.3 .3 .1)\end{array}$ & $\begin{array}{c}\text { Cloud Computes } \\
\qquad(\$ 4.3 .3 .2)\end{array}$ & $\begin{array}{c}\mathcal{T} \text { Sets Up and } \\
\text { Cloud Computes } \\
(\$ 4.3 .3 .3)\end{array}$ \\
\hline Computation by $\mathcal{C}$ & $\mathcal{O}\left(\left|x_{i}\right|+\left|y_{i}\right|\right)$ & $\mathcal{O}\left(\left|x_{i}\right|+\left|y_{i}\right|\right)$ & $\mathcal{O}\left(\left|x_{i}\right|+\left|y_{i}\right|\right)$ \\
\hline Communication $\mathcal{C} \leftrightarrow \mathcal{S}$ & $\mathcal{O}\left(\left|x_{i}\right|+\left|y_{i}\right|\right)$ & $\mathcal{O}\left(\left|x_{i}\right|+\left|y_{i}\right|\right)$ & $\mathcal{O}\left(\left|x_{i}\right|+\left|y_{i}\right|\right)$ \\
\hline Storage in Cloud & $\mathcal{O}(|f|+|D|)$ & $\mathcal{O}(|f|+|D|)$ & $\mathcal{O}(|f|+|D|)$ \\
\hline Computation by $\mathcal{T}$ & $\mathcal{O}(|f|)$ (Online) & none & $\mathcal{O}(|f|)$ (Setup) \\
\hline Computation by Cloud & none & $\mathcal{O}(|f|)$ (Online) & $\mathcal{O}(|f|)$ (Online) \\
\hline Online Latency & $\mathcal{T}$ evals $f$ & Cloud evals $\widetilde{f}(\llbracket \cdot \rrbracket)$ & Cloud evals $\widetilde{f}(\cdot)$ \\
\hline
\end{tabular}




\section{Chapter 5}

\section{Modular Design of Efficient SFE Protocols}

For several years, two approaches for two-party Secure Function Evaluation (SFE) have co-existed - based on either Homomorphic Encryption (HE) (cf. \$2.2.1) or Garbled Circuits (GCs) (cf. \$2.3.1). Both approaches have their respective advantages and disadvantages, e.g., GC requires to transfer the Garbled Circuit (communication complexity is at least linear in the size of the function) but allows to pre-compute almost all expensive operations resulting in a low latency of the online phase, whereas most HE schemes require relatively expensive public-key operations in the online phase but can result in a smaller overall communication complexity. For a particular primitive, one of the techniques is usually more suitable than the other. For example, for comparisons or computing the maximum, $\mathrm{GC}$ is better than $\mathrm{HE}$ as described in $\$ 3.4 .1$, whereas multiplication can benefit from using $\mathrm{HE}$ as described in \$5.2.5.1. Therefore, simply switching from one approach for secure computation to the other can result in substantial performance improvements. For instance, for privacy-preserving DNA matching based on secure evaluation of finite automatons, the GC-based protocol [Fri09] is more efficient than the HE-based version [TPKC07].

Combination of Efficient SFE techniques. Going one step further, it would be beneficial to use the most efficient primitive for the respective sub-task even if they are based on different SFE paradigms. Indeed, secure and efficient composition of sub-protocols based on $\mathrm{HE}$ and $\mathrm{GC}$ can result in performance improvements as shown for several privacy-preserving applications (e.g., [BPSW07, BS09, BFK ${ }^{+}$09b, SSW09]).

Outline. In this chapter we present a framework to modularly combine efficient SFE protocols based on $\mathrm{GC}$ and $\mathrm{HE}$ in $\$ 5.1$. This framework has been implemented in the Tool for Automating Secure Two-partY computations (TASTY) which we describe in $\$ 5.2$. As application we consider privacy-preserving face recognition in $\$ 5.3$. 


\subsection{Framework for Modular SFE Protocols}

In this section we describe our framework for modularly composing efficient Secure Function Evaluation (SFE) protocols based on Garbled Circuit (GC) and Homomorphic Encryption (HE). We give instantiations of the primitives and conversions of our framework secure in the semi-honest model.

Publication Info: Parts of the following results are published in $\mathrm{BFK}^{+} 09 \mathrm{~b}, \mathrm{KSS} 09$, KSS10] and [BFL ${ }^{+} 11$, Sect. IV].

\subsubsection{Function Representations}

As the complexity of today's most efficient SFE protocols depends linearly on the size of the evaluated function, an obvious approach to improve efficiency is to look for a small representation of the function to be evaluated. However, it is not feasible to describe the optimal choice strategy as finding minimal function representations is hard [BW96, $\mathrm{KC00]}$.

Standard representations for functions which are particularly useful for SFE are boolean circuits (cf. \$2.1.3.1) and arithmetic circuits (cf. \$2.1.3.2).

Ordered Binary Decision Diagrams (OBDDs), Another function representation are OBDDs. These can be used to encode decision strategies in a compact way, whereas other functions such as multiplication require exponentially large OBDDs Bry91, Woe05. Protocols for SFE of a function represented as OBDD are similar to Yao's GC protocol for SFE of boolean circuits, except that they construct and evaluate a garbled OBDD instead of a GC. We do not consider OBDDs explicitly in the following description of our framework, but note that they can be used similarly to GCs. Methods for constructing garbled OBDDs were described first in [KJGB06] and improved in [Sch08, Sect. 3.4.1] and [BFK ${ }^{+}$09b].

Hybrid Functions. In our framework described below, we advocate a hybrid approach, where function blocks can be represented either way, i.e., as boolean circuit, arithmetic circuit, or OBDD. These blocks are evaluated using the corresponding SFE technique, and their encrypted intermediate results then glued together. This allows to choose the most efficient SFE technique for a specific sub-functionality. For example multiplication of $n$-bit integer values requires only a single multiplication gate in an arithmetic circuit over a sufficiently large ring, whereas the boolean circuit has size $\mathcal{O}\left(n^{2}\right)$ using school method or $\mathcal{O}\left(n^{\log _{2} 3}\right)$ with the method of [KO62] as described in 3.3.2. We determine experimentally which method for secure multiplication is the most efficient for a specific input length in 55.2 .5 .1 . 


\subsubsection{Modular SFE}

In $\$ 2$ we have described how arithmetic circuits can be evaluated securely using HE (\$2.2.1.3) and boolean circuits using GCs (2.3.1.1). In the following we give a universal framework that combines both approaches and allows to convert back and forth between them. This allows arbitrary compositions of both techniques and implies significant improvements to SFE.

The basic idea of our modular SFE framework is to compose SFE protocols as a sequence 1 of operations on encrypted data as shown in Fig. 5.1. both parties have Plain Values as their inputs into the protocol. These plain values, denoted as $x$, are first encrypted by converting them into their corresponding encrypted value: a Garbled Value, denoted as $\widetilde{x}$, held by $\mathcal{C}$, or a Homomorphic Value, denoted as $\llbracket x \rrbracket$ held by $\mathcal{S}$, depending on which operations should be applied. After encryption, the function is securely evaluated on the encrypted values, which may involve conversion of the encryptions into the respective other type of encryption (cf. \$5.1.3). Finally, the encrypted output values are revealed and can be decrypted by converting them into the corresponding plain output values.

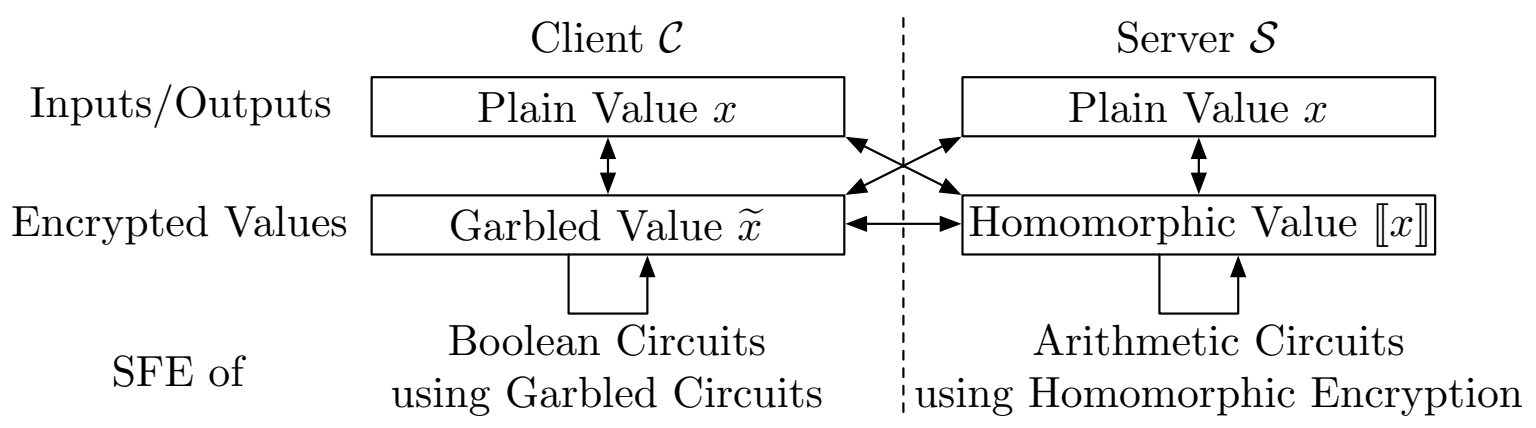

Figure 5.1: Hybrid SFE Protocols

In the following we define the two types of encryptions, garbled values (\$5.1.2.1) and homomorphic values $(\$ 5.1 .2 .2$ and describe their conversions from and to plain values. Afterwards we describe methods for converting between both types of encryptions in $\$ 5.1 .3$.

\subsubsection{Garbled Values and Conversions}

The interface for GC-based SFE protocols are garbled values (cf. Fig. 5.1). As described in 2.2 .2 .1 , a garbled boolean value $\widetilde{x}_{i}=\left\langle k_{i}, \pi_{i}\right\rangle$ represents a bit $x_{i}$. It consists

\footnotetext{
${ }^{1}$ As all building blocks are secure against semi-honest adversaries, their sequential composition inherits this security property (see e.g., Gol04]).
} 
of a key $k_{i} \in\{0,1\}^{t}$, where $t$ is the symmetric security parameter (cf. 2.1.1.2), and a permutation bit $\pi_{i} \in\{0,1\}$. The garbled value $\widetilde{x}_{i}$ is assigned to one of the two corresponding garbled values $\widetilde{x}_{i}^{0}=\left\langle k_{i}^{0}, \pi_{i}^{0}\right\rangle$ or $\widetilde{x}_{i}^{1}=\left\langle k_{i}^{1}, \pi_{i}^{1}\right\rangle$ with $\pi_{i}^{1}=1-\pi_{i}^{0}$. A garbled $\ell$-bit value can be viewed as a vector of $\ell$ garbled boolean values.

We show how to convert a plain value into its corresponding garbled value and back next.

Plain Value to Garbled Value for Inputs. To translate a plain value $x_{i}$ held by $\mathcal{S}$ into a garbled value $\widetilde{x}_{i}$ for $\mathcal{C}, \mathcal{S}$ sends the corresponding garbled value $\widetilde{x}_{i}^{0}$ or $\widetilde{x}_{i}^{1}$ to $\mathcal{C}$ depending on the value of $x_{i}$.

To convert a plain value $x_{i}$ held by $\mathcal{C}$ into a garbled value $\widetilde{x}_{i}$ for $\mathcal{C}$, both parties execute an Oblivious Transfer (OT) protocol where $\mathcal{C}$ inputs $x_{i}, \mathcal{S}$ inputs $\widetilde{x}_{i}^{0}$ and $\widetilde{x}_{i}^{1}$ and the output to $\mathcal{C}$ is $\widetilde{x}_{i}=\widetilde{x}_{i}^{0}$ if $x_{i}=0$ or $\widetilde{x}_{i}^{1}$ otherwise. OT can be implemented efficiently as described in 2.2 .3 .

Garbled Value to Plain Value for Outputs. To convert a garbled value $\widetilde{x}_{i}=$ $\left\langle k_{i}, \pi_{i}\right\rangle$ into its corresponding plain value $x_{i}$ for $\mathcal{C}, \mathcal{S}$ reveals the output permutation bit $\pi_{i}^{0}$ which was used during construction of the garbled wire and $\mathcal{C}$ obtains $x_{i}=\pi_{i} \oplus \pi_{i}^{0}$.

If the garbled value $\widetilde{x}_{i}$ should be converted into a plain value for $\mathcal{S}, \mathcal{C}$ can simply send $\widetilde{x}_{i}$ to $\mathcal{S}$ who obtains the plain value by decrypting it, e.g., compare with $\widetilde{x}_{i}^{0}$ and $\widetilde{x}_{i}^{1}$. We note that malicious $\mathcal{C}$ cannot cheat in this conversion as she only knows one of the two garbled values possible and is unlikely to guess the other one. Alternatively, if $\mathcal{C}$ is assumed to be semi-honest, it suffices to send $\pi_{i}$ to $\mathcal{S}$ who obtains $x_{i}=\pi_{i} \oplus \pi_{i}^{0}$.

\subsubsection{Homomorphic Values and Conversions}

The interface for HE-based SFE protocols are homomorphic values, i.e., HEs held by $\mathcal{S}$ and encrypted under the public key of $\mathcal{C}$ (cf. Fig. 5.1). These homomorphic values can be converted from or to plain values as described next.

Plain Value to Homomorphic Value for Inputs. To convert a plain $\ell$-bit value $x$ into a homomorphic value $\llbracket x \rrbracket, x$ held by $\mathcal{S}$ is simply encrypted under $\mathcal{C}$ 's public key. If $x$ belongs to $\mathcal{C}, \llbracket x \rrbracket$ is sent to $\mathcal{S}$.

Homomorphic Value to Plain Value for Outputs. To convert a homomorphic value into a plain value for $\mathcal{C}, \mathcal{S}$ sends the homomorphic value to $\mathcal{C}$ who decrypts and obtains the plain value. If only $\mathcal{S}$ should learn the plain value corresponding to a homomorphic $\ell$-bit value $\llbracket x \rrbracket, \mathcal{S}$ additively blinds the homomorphic value by choosing a random mask $r \in_{R}\{0,1\}^{\ell+\sigma}$, where $\sigma$ is the statistical security parameter (cf. $\$ 2.1 .1 .2$, and computing $\llbracket \bar{x} \rrbracket=\llbracket x \rrbracket \boxplus \llbracket r \rrbracket . \mathcal{S}$ sends this blinded value to $\mathcal{C}$ who decrypts and sends back $\bar{x}$ to $\mathcal{S}$. Finally, $\mathcal{S}$ computes $x=\bar{x}-r$. 
Packing (cf. \$2.2.1.3 can be used to improve efficiency of parallel output conversions.

Multiplication of Homomorphic Values with Additively-Homomorphic Encryption. If the $\mathrm{HE}$ scheme is only additively homomorphic, two homomorphic values can be multiplied with a single round of interaction as described in \$2.2.1.3. Efficiency of parallel multiplications can be improved by packing multiple blinded ciphertexts together.

\subsubsection{Conversion between Encrypted Values}

The main idea for converting a homomorphic value $\llbracket x \rrbracket$ into a garbled value $\widetilde{x}$ is that addition is a relatively cheap operation under both types of encryptions: One party chooses a random mask and additively blinds the encrypted value. The blinded value is converted into a plain value and encrypted with the target scheme. Finally, the random mask is taken off under encryption.

We give details on the conversion protocols next.

\subsubsection{Garbled Values to Homomorphic Values}

A garbled $\ell$-bit value $\widetilde{x}$ held by $\mathcal{C}$ (usually obtained from evaluating a GC) can be efficiently converted into a homomorphic value held by $\mathcal{S}$ by using additive blinding or bitwise encryption as described next.

Additive Blinding. $\mathcal{S}$ randomly chooses a random mask $r \in_{R}\{0,1\}^{\ell+\sigma}$, where $\sigma$ is the statistical security parameter (cf. 82.1 .1 .2 ) and $\ell+\sigma \leq|P|$ to avoid an overflow, and adds the random mask converted into garbled value $\widetilde{r}$ to $\widetilde{x}$ using a garbled $(\ell+\sigma)$ bit addition circuit (cf. $\$ 3.3 .1 .1$ that computes $\widetilde{\bar{x}}$ with $\bar{x}=x+r$. This value is converted into a plain output value $\bar{x}$ for $\mathcal{C}$ who homomorphically encrypts this value and sends the result $\llbracket \bar{x} \rrbracket$ to $\mathcal{S}$. Finally, $\mathcal{S}$ takes off the random mask under encryption as $\llbracket x \rrbracket=\llbracket \bar{x} \rrbracket \boxplus(-1) \llbracket r \rrbracket$. A detailed description of this conversion protocol is given in [KSS09].

Bitwise Encryption. If the bit length $\ell$ of $\widetilde{x}$ is small, a bitwise approach can be used as well in order to avoid the garbled addition circuit: $\mathcal{C}$ homomorphically encrypts the permutation bits $\pi_{i}$ of the garbled boolean output values $\widetilde{x}_{i}=\left\langle k_{i}, \pi_{i}\right\rangle$ and sends $\llbracket \pi_{i} \rrbracket$ to $\mathcal{S}$. $\mathcal{S}$ flips those encrypted permutation bits for which the permutation bit was set as $\pi_{i}^{0}=1$ during creation to $\llbracket \pi_{i}^{\prime} \rrbracket=\llbracket 1 \rrbracket \boxplus(-1) \llbracket \pi_{i}^{\prime} \rrbracket$ or otherwise sets $\llbracket \pi_{i}^{\prime} \rrbracket=\llbracket \pi_{i} \rrbracket$. Then, $\mathcal{S}$ combines these bit encryptions $\llbracket \pi_{i}^{\prime} \rrbracket$ using Horner's scheme as $\llbracket x \rrbracket=\llbracket \pi_{\ell}^{\prime}\|..\| \pi_{1}^{\prime} \rrbracket$. 
Performance Comparison. The conversion based on additive blinding requires a garbled addition circuit (cf. \$3.3.1.1) for $(\ell+\sigma)$-bit values and the transfer of the $(\ell+\sigma)$-bit garbled value $\widetilde{r}$. When using Garbled Row Reduction of [NP01] and free XORs of [KS08a] as described in $\$ 2.2 .2 .3$, this requires in total $(3+1)(\ell+\sigma)(t+1)$ bits sent from $\mathcal{S}$ to $\mathcal{C}$ in the pre-computation phase. In the online phase, the GC is evaluated and the result is homomorphically encrypted and sent to $\mathcal{S}$ (one ciphertext).

The conversion using bitwise encryption requires $\ell$ HEs and transfer of $\ell$ ciphertexts from $\mathcal{C}$ to $\mathcal{S}$ in the online phase. At least for converting a single bit, i.e., when $\ell=1$, this technique results in better performance.

\subsubsection{Homomorphic Values to Garbled Values}

In the following we describe how to convert a homomorphic $\ell$-bit value $\llbracket x \rrbracket$ into a garbled value $\widetilde{x}$. This protocol has been widely used to combine HE and GC, e.g., in [BPSW07, BS09, JP09, BFK ${ }^{+}$09b].

$\mathcal{S}$ additively blinds $\llbracket x \rrbracket$ with a random pad $r \in_{R}\{0,1\}^{\ell+\sigma}$, where $\sigma$ is the statistical security parameter (cf. $\$ 2.1 .1 .2$ ) and $\ell+\sigma \leq|P|$ to avoid an overflow, as $\llbracket \bar{x} \rrbracket=\llbracket x \rrbracket \boxplus \llbracket r \rrbracket$. $\mathcal{S}$ sends the blinded ciphertext $\llbracket \bar{x} \rrbracket$ to $\mathcal{C}$ who decrypts and inputs the $\ell$ least significant bits of $\bar{x}, \chi=\bar{x} \bmod 2^{\ell}$, to an $\ell$-parallel OT protocol (cf. $\$ 2.2 .3$ ) to obtain the corresponding garbled value $\tilde{\chi}$. Then, the mask is taken off within a garbled $\ell$-bit subtraction circuit (cf. $\$ 3.3 .1 .2$ ) which gets as inputs $\tilde{\chi}$ and $\widetilde{\rho}$ converted from $\rho=r$ $\bmod 2^{\ell}$ as input from $\mathcal{S}$. The output obtained by $\mathcal{C}$ is $\widetilde{x}$ which corresponds to $x=\chi-\rho$.

As proposed in $\left[\mathrm{BFK}^{+} 09 \mathrm{~b}\right]$, packing as described in $\$ 2.2 .1 .3$, can be used to improve parallel conversions from homomorphic to garbled values by packing multiple ciphertexts together before additive blinding and sending them to $\mathcal{C}$.

\subsection{Compiling Modular SFE Protocols}

In this section we present TASTY, a novel tool for automating, i.e., describing, generating, executing, benchmarking, and comparing, efficient secure two-party computation

protocols. TASTY is a new compiler that implements the modular Secure Function Evaluation (SFE) framework described in $\$ 5.1$ and can generate protocols based on Homomorphic Encryption (HE) and efficient Garbled Circuit (GC) as well as combinations of both, which often yields the most efficient protocols available today. The user provides a high-level description of the computations to be performed on encrypted data in a domain-specific language. This is automatically transformed into a protocol. TASTY provides most recent techniques and optimizations for practical secure twoparty computation with low online latency (cf. \$2.2). Moreover, it allows to efficiently evaluate circuits generated by the well-known Fairplay compiler [MNPS04, BDNP08].

We use TASTY to compare protocols for secure multiplication based on $\mathrm{HE}$ with those based on GCs and fast multiplication (cf. \$3.3.2.2). Further, we show how 
TASTY improves the online latency for securely evaluating the AES functionality by an order of magnitude compared to previous software implementations. TASTY allows to automatically generate efficient secure protocols for many privacy-preserving applications where we consider the use case of privacy-preserving face recognition in $\$ 5.3$.

Publication Info: Parts of the following results are published in $\left[\mathrm{HKS}^{+} 10\right]$.

\subsubsection{Introduction}

In the following, we motivate TASTY $(\$ 5.2 .1 .1)$, give the outline and our contribution $(\$ 5.2 .1 .2)$, and present related works $(\$ 5.2 .1 .3)$.

\subsubsection{Motivation}

The design of efficient two-party SFE protocols is vital for a variety of security-critical applications with sophisticated privacy and security requirements such as electronic auctions [NPS99], data mining [LP09b], remote diagnostics [BPSW07], classification of medical data $\left[\mathrm{BFK}^{+} 09 \mathrm{~b}\right]$, or face recognition $\left[\mathrm{EFG}^{+} 09\right.$, SSW09, OPJM10] to name some.

Modern cryptography provides various tools for secure computation. The concept of two-party SFE was introduced in 1982 by Yao Yao82. The idea is to let two mutually mistrusting parties compute an arbitrary function (known by both) on their private inputs without revealing any information about their inputs beyond the function's output (cf. \$2 for details). However, the real-world deployment of SFE was believed to be very limited and expensive for a relatively long time. Fortunately, the cost of SFE has been dramatically reduced in the recent years thanks to many algorithmic improvements and automatic tools, as well as faster computing platforms and communication networks.

In the recent years several cryptographic compilers and specification languages have been proposed that, after a programmer has manually mapped an existing algorithm to integer arithmetics, automatically compile a high-level program into a corresponding SFE protocol. We will give an overview on such previous works in \$5.2.1.3. However, such tools are currently restricted to generating protocols based on only one SFE paradigm, i.e., use either GCs or HE, which often results in protocols with suboptimal efficiency. For instance $\mathrm{HE}$ allows efficient addition and multiplication of large values (as confirmed by our implementation results in \$5.2.5.1), whereas GCs are better for non-linear functionalities such as comparison (cf. \$3.4.1). As shown in \$5.1, combining both approaches allows to obtain relatively efficient protocols when designing privacypreserving applications, e.g., remote diagnostics [BPSW07], classification [BFK $\left.{ }^{+} 09 \mathrm{~b}\right]$, or face recognition [SSW09]. 
TASTY is the first compiler that can automatically generate efficient protocols based on $\mathrm{HE}$ and GCs as well as combinations of both from a high-level description of the protocol.

\subsubsection{Outline and Contribution}

In summary, TASTY realizes and experimentally verifies many of the concepts and optimizations presented in this thesis. The remainder of this chapter presents the following contributions in the respective sections.

SFE Compiler. We present TASTY, a tool that allows to automatically generate, benchmark and compare the performance of efficient two-party SFE protocols in the semi-honest model (\$5.2.2). We show how TASTY is related to, improves over, and can be combined with existing tools for automatic generation of (two-party) SFE protocols $(\$ 5.2 .1 .3$.

Specification Language. The TASTY input Language (TASTYL) allows to describe SFE protocols as sequence of operations on encrypted data based on combinations of GCs and HE. Hence, it provides a compact and user-friendly description language for describing protocols in the modular SFE framework of \$5.1. TASTYL is based on the Python programming language and hides technical cryptographic details from the programmer $(5.2 .3)$.

Efficient Building Blocks. TASTY implements efficient building blocks for HE and $\mathrm{GC}$ which allow to shift most of the complexity into the less time critical setup phase resulting in SFE protocols with a low-latency online phase (\$5.2.4). While the implemented techniques have been known before, their combination and implementation in a single package is unique and useful. We show how the combination of these techniques speeds up the online phase for secure evaluation of AES (a large circuit with more than 30,000 gates) compared to the currently fastest software implementation of GCs PSSW09] from $5 \mathrm{~s}$ to only $0.5 \mathrm{~s}$, while the total costs for setup plus online phase stay almost the same $(\$ 5.2 .5 .2$.

Circuit Optimizations. Additionally, TASTY has built-in tools for on-the-fly generation and minimization of boolean circuits (\$5.2.4). As new circuit building block we implement fast multiplication circuits based on Karatsuba's method [KO62] described in 3.3 .2 .2 . We show that this is more efficient than textbook multiplication (used in previous SFE tools) already for 20 bit numbers.

Benchmarking. Using TASTY, we obtain measurements for a detailed performance comparison of multiplication protocols based on GCS with those based on HE. Our 
experiments show that GC-based multiplication has large communication and time complexity in the setup phase, but results in a more efficient online time than $\mathrm{HE}$. based multiplication for small values (\$5.2.5.1). In particular, multiplication of two garbled values with bit length $\ell \leq 16$ bits requires less online communication and time than the multiplication of two homomorphically encrypted values for short-term security parameters.

Applications. TASTY is a usable and useful tool for describing and automatically generating efficient protocols for several privacy-preserving applications. As representative example we concentrate on privacy-preserving face recognition in $\$ 5.3$. Further application examples are secure set intersection [FNP04, LP09b, $\mathrm{HKS}^{+}$10] and privacypreserving medical diagnostics $\left[\mathrm{BFK}^{+} 09 \mathrm{~b}, \mathrm{BFK}^{+}\right.$09a, SS09, SS10a].

\subsubsection{Existing Tools for Two-Party SFE}

While the theoretical foundations of two-party SFE have been laid already in the eighties Yao82, Yao86, recent optimizations and prototype implementations show that SFE is ready to be used in practical applications (e.g., [LPS08, [PSSW09]). To allow the deployment of SFE in a wide range of privacy-preserving applications it is not only important to maximize the efficiency of SFE protocols, but also to make SFE usable by automatically generating protocols from high-level descriptions. For this, several frameworks for SFE consisting of languages and corresponding tools have been developed in the last years. We review these proposals briefly in the following.

Existing SFE frameworks can be divided into three classes on different abstraction levels as summarized in Table 5.1 .

Table 5.1: Abstraction Levels: Automatic Generation of SFE Protocols

\begin{tabular}{|l|l|}
\hline Abstraction Level & Primitives \\
\hline \hline Function Description & I/O, computation \\
\hline Protocol Description & I/O, enc/dec, computation under encryption \\
\hline Protocol Implementation & I/O, protocols, messages, crypto primitives \\
\hline
\end{tabular}

Function Description languages allow to specify what function should be computed securely. The function is described in a domain-specific high-level programming language which allows programmers to write programs using SFE without any expert knowledge about SFE. Functions described in such languages can then be (formally) analyzed to ensure security of the function (e.g., no information leak to the other party) and are compiled (potentially through lower-level SFE languages) into 
SFE protocols. Examples are Fairplay's Secure Function Definition Language (SFDL) [MNPS04, BDNP08] which can be compiled to boolean circuits (see below), or the Secure Multiparty Computation Language (SMCL) [NS07] and its Python-based successor PySMCL [Nie09] which allow compilation into arithmetic circuit-based Secure Multi-Party Computation (SMPC) protocols such as the Virtual Ideal Functionality Framework (VIFF) [DGKN09].

Protocol Description languages allow to specify how the SFE protocol is composed as sequence of basic operations on encrypted (or secret-shared data). Examples (described in more detail below) are VIFF [DGKN09, the Secure Multiparty Computation language (SMC) [NSY04, Sil04], Sharemind [BLW08], and the compiler of MacKenzie et al. [MOR03]. These languages allow to specify SFE protocols while abstracting away the details of the underlying cryptographic protocols. The language and compiler we present in this section also fall into this class. However, in contrast to previous works which were restricted to using HE only, our compiler TASTY allows arbitrary combinations of computations under encryption based on GCs and/or HE for highly efficient SFE protocols.

Protocol Implementation languages allow to describe how exactly the target SFE protocol is composed as sequence of basic cryptographic protocol building blocks. They reside at the lowest level of the abstraction hierarchy and require a substantial amount of expert knowledge in cryptographic protocol design. For example the L1 language [SKB ${ }^{+}$09, SKM10] allows to describe secure computation protocols as sequence of basic primitives such as Oblivious Transfer (OT), encryption/decryption, creation and evaluation of GCs, and messages to be exchanged. Qilin [Mor] is a Java library for rapid prototyping of cryptographic protocols which currently provides common cryptographic protocols (e.g., OT NP01 and coin flipping) using cryptographic primitives (e.g., Pedersen Commitment [Ped92] and ElGamal Gam85]) implemented with Elliptic Curves (ECs).

Next we describe SFE frameworks which are closely related to ours. In contrast to TASTY, the existing SFE frameworks are based on either GCs or $\mathrm{HE}$, but not combinations of both.

Garbled Circuits (GCs), The most prominent example for automatic generation of SFE protocols is Fairplay [MNPS04] which is based on GCs. Fairplay provides a high-level function description language, SFDL, which allows to specify the function to be computed securely, i.e., the inputs and outputs of the involved parties, and how the outputs are to be computed from the inputs. The language resembles a simplified version of a hardware description language, such as Verilog or Very high speed integrated circuit Hardware Description Language (VHDL). It supports types, variables, 
functions, boolean operators $(\wedge, \vee, \oplus, \ldots)$, arithmetic operators $(+,-)$, comparison $(<, \geq,=, \ldots)$ and control structures like if-then-else or for-loops with constant range. The Fairplay compiler compiles and optimizes an SFDL program into a boolean circuit which is stored in a file. The circuit can then be evaluated using the Fairplay runtime environment, two Java programs which securely evaluate the circuit using Yao's GC protocol, communicating over a TCP socket. Fairplay is supplemented by FairplayMP [BDNP08], a multi-party version of Fairplay suited for three or more parties with the more powerful SFDL 2 input language (with support for $*, /$ and generic functions) and a corresponding circuit compiler. TASTY can serve as efficient runtime environment for the Fairplay compiler suite, i.e., it allows to read in circuits generated by the FairplayMP compiler from SFDL 2 programs $2^{2}$ and optimizes these for efficient secure evaluation with state-of-the-art GC evaluation techniques.

Homomorphic Encryption (HE), VIFF [DGKN09], the Virtual Ideal Functionality Framework, is an open source framework written in Python for specifying SMPC protocols as a sequence of operations performed on secret-shared (i.e., encrypted) data. While VIFF was mainly designed for secret-sharing based SMPC protocols with three or more parties, it also offers a two-player runtime based on the additively homomorphic Paillier cryptosystem [Pai99]. Using operator overloading, VIFF allows the programmer to express a desired secure computation directly as standard arithmetic without knowing about the used protocol. Indeed, TASTYL, the input language of our compiler, is inspired by the VIFF language, but additionally allows to combine HE with GC-based computations.

In contrast to general-purpose compilers such as Fairplay, VIFF, and TASTY, the compilers described below are built for specific application scenarios, e.g., use specific number representations [MOR03, BLW08] or require $n \geq 3$ parties [NSY04, Sil04, BLW08]: The compiler of MacKenzie et al. [MOR03] implements secure two-party computations over values which are secret-shared between both parties using $\left(\begin{array}{l}2 \\ 2\end{array}\right)$ secret-sharing over a prime field. The computations are composed as sequence of basic operations on the shared data (e.g., addition or multiplication). The compiler can be used for specific functions such as cryptographic primitives defined over prime fields, e.g., signatures or encryption schemes, where the secret key is shared between both parties.

SMC [NSY04, Sil04], the Secure Multiparty Computation language, provides a declarative language for describing SMPC based on constraint programming. A program is distributed among the parties in the computation along with an interpreter, each party gives its secret inputs and the interpreter calculates the result. Computations are specified as arithmetic circuits and at least 3 parties are required as the underlying multiplication protocol is based on the BGW protocol [BGW88].

Sharemind [BLW08] allows secure computation over the ring of 32-bit integers for three parties and provides an assembly-like programming language. As this setting is fixed

\footnotetext{
${ }^{2}$ FairplayMP's compiler can also be used to generate circuits for two parties.
} 
and very specific it allows highly efficient protocols.

\subsubsection{Tool for Automating Secure Two-partY computations (TASTY)}

In the following we present TASTY, our tool for describing and automatically generating, benchmarking, and evaluating hybrid two-party SFE protocols.

Design Goals. TASTY was designed and developed to meet the following goals:

1. SFE protocols are programmed in TASTYL, an intuitive high-level language for describing the protocol as sequence of operations on encrypted data (cf. \$5.2.3).

2. TASTYallows to test, benchmark, and compare the performance of the generated $\mathrm{SFE}$ protocols (cf. [HKS ${ }^{+}$10] for details).

3. The generated SFE protocols aim at minimizing the latency of the online phase, i.e., the time from providing the inputs until obtaining the outputs. This is achieved by using a combination of highly efficient primitives and pre-computations (cf. 55.2 .4 ).

Architecture and Workflow (cf. Fig. 5.2). The workflow for using TASTY is as follows:

1. Both users, client $\mathcal{C}$ and server $\mathcal{S}$, agree on a Protocol Description of the SFE protocol in the TASTY input Language (TASTYL) as described in detail in $\$ 5.2 .3$.

2. Both users invoke TASTY ${ }^{\text {T }}$ Runtime Environment (details in $\left[\mathrm{HKS}^{+} 10\right]$ ), a program that can automatically analyze, run, test, and benchmark the SFE protocol:

a) In the Analyzation Phase, the runtime environment checks the syntactical correctness of the protocol description, exchanges a hash of it to ensure that both parties run the same protocol, and analyzes the protocol to automatically determine which parts of the protocol can be pre-computed.

b) In the Setup Phase, the parties pre-compute those parts of the protocol which are independent of their inputs, e.g., create/send GCs and precompute OTs (cf. \$5.2.4 for details).

c) Finally, in the Online Phase, both parties provide their inputs to the computation, and the online part of the SFE protocol is executed (e.g., encryptions and decryptions, online OTs, and evaluation of GCs to jointly compute the respective outputs for both parties. 
3. TASTY provides a tool to compare the performance costs of multiple SFE protocols (cf. [HKS ${ }^{+}$] for details).

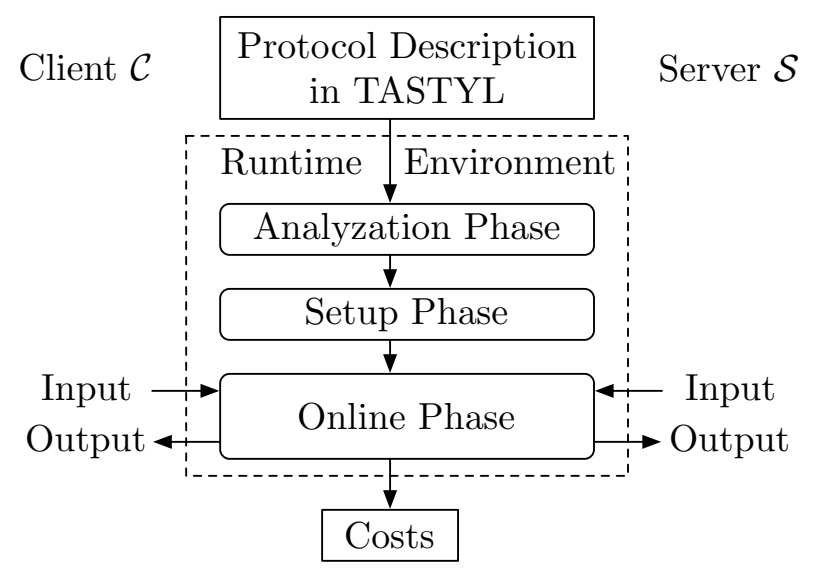

Figure 5.2: Architecture and Workflow of TASTY

Implementation. As implementation language for TASTY we selected Python Pyt as it combines elements from both, object oriented and functional programming paradigms. In particular the built-in support for generators, a function which yields a value and can be resumed afterwards, was useful for intuitive programming of streamlined large data structures, e.g., for dynamic generation of circuits which allows TASTY to generate and evaluate very large circuits with low memory footprint.

\subsubsection{TASTY input Language (TASTYL)}

TASTYL, the input language for TASTY, allows to formulate secure computations as sequence of operations on encrypted data, allowing to abstract away all details of the underlying cryptographic protocols. We start with an overview of the types and operators provided by TASTYL in $\$ 5.2 .3 .1$ and explain the concrete syntax afterwards in $\$ 5.2 .3 .2$.

\subsubsection{TASTYL Types and Operators}

The type system of TASTYL and the operators supported by each type are shown in Fig. 5.3. Each variable in TASTYL is either a scalar Value (cf. top half of Fig. 5.3) or a Vector (cf. bottom half of Fig. 5.3) which consists of $N$ Values. They can be either unencrypted Plain Values/Vectors or encrypted Garbled or Homomorphic Values/Vectors. 


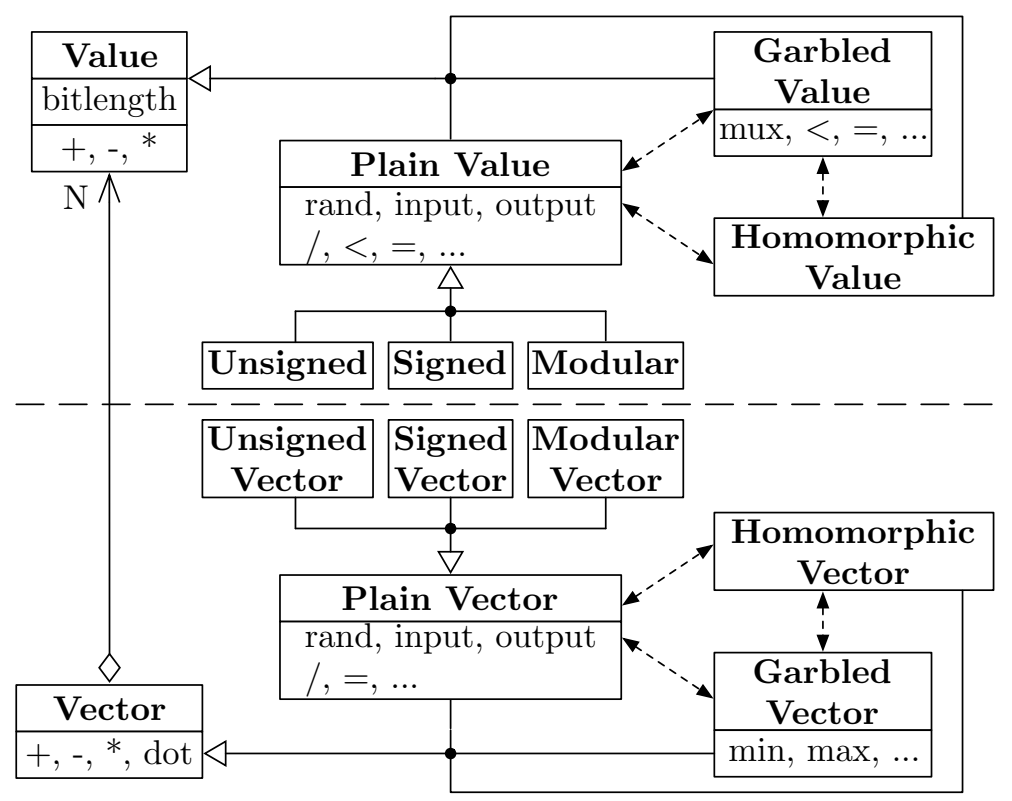

Figure 5.3: TASTYL: Types and Operators

All Values and Vectors provide the basic operators for (component-wise) addition, subtraction, and multiplication; Vectors also provide dot multiplication: $\boldsymbol{v} \cdot \boldsymbol{w}=$ $\sum_{i=1}^{N} v_{i} w_{i}$.

Number Representation. Each Value has a bit length $\ell$ that represents the number of bits needed for its representation. Unsigned are unsigned integer values in the range $\left[0,2^{\ell}[\right.$, Signed are signed integers in the range $]-2^{\ell-1}, 2^{\ell-1}[3$, and Modular are elements in the plaintext space of the homomorphic cryptosystem, i.e., $\mathbb{Z}_{n}$ for Paillier.

In addition to the operations of Value/Vector, the plain/encrypted types support further operations and conversions:

Plain Value/Vector. Inputs and outputs of the two parties are Plain Values/Vectors. They can be chosen uniformly at random and provide additional operations (integer) division ${ }^{4}$ and comparison.

Homomorphic Value/Vector. Unsigned, Signed and Modular Values/Vectors can be converted into and from homomorphically encrypted Homomorphic Values/Vectors of server $\mathcal{S}$. Unsigned and Modular values are mapped directly to $0, \ldots, n-1$. For

\footnotetext{
${ }^{3}$ Note, we exclude the value $-2^{\ell-1}$ for signed integers to also allow sign-magnitude representation.

${ }^{4}$ Division raises an exception for division by zero or (the unlikely event of) a non-invertible Modular value.
} 
Signed values, the positive values are mapped to the elements $0,1, \ldots$ of the plaintext space of the underlying homomorphic cryptosystem, and the negative values to $n-1, n-2, \ldots$ as described in [KSS10]. Addition of two Homomorphic, and (dot) multiplication of a Homomorphic with a Plain Value/Vector provided by $\mathcal{S}$ is done non-interactively. (Dot) multiplication of two Homomorphic Values/Vectors requires one round of interaction (cf. $\$ 2.2 .1 .3$ ).

Garbled Value/Vector. Unsigned/Signed Plain and Homomorphic Values/Vectors can be converted into and from Garbled Values/Vectors of client $\mathcal{C}$. A Garbled Value can be compared with another one resulting in a Garbled Value of length one bit. This can be used to multiplex (mux), i.e., choose one out of, two Garbled Values. Similarly, the minimum or maximum value and/or index of the components of a Garbled Vector can be determined as Garbled Value(s), e.g., min_value computes the minimum value. For each operation on Garbled Values/Vectors, TASTY automatically infers the underlying $\mathrm{GC}$ (cf. §3.3).

\subsubsection{TASTYL Syntax and Example}

TASTYL is a subset of the Python language; we use the following example to explain its syntax and semantics.

Example 9 (TASTYL Example). Client $\mathcal{C}$ and server $\mathcal{S}$ have vectors $\boldsymbol{v}$ and $\boldsymbol{w}$ of $N=4$ unsigned 32 -bit values as inputs. As output, $\mathcal{C}$ obtains $\min _{i=1, \ldots, N}\left(v_{i} \cdot w_{i}\right)$. The products $v_{i} \cdot w_{i}$ are computed with $\mathrm{HE}$ and the minimum with GCs.

This protocol can be directly formulated in TASTYL as shown in Fig. 5.4 and described in the following: The protocol gets two parties client and server as inputs to whom the variables used throughout the protocol are bound (details below). At the beginning, two constants $N=4$ and $L=32$ are defined. Then, the input of $\mathcal{C}$, client.v, is defined as an unsigned vector of bit length $L$ and dimension $N$, and read from standard input. Similarly, the input of $\mathcal{S}$, server.w, is defined and read. Then, $\mathcal{C}$ 's input vector client.v is converted into a homomorphic vector server.hv for $\mathcal{S}$ who multiplies this component-wise with his input vector server.w resulting in the homomorphic vector server. $\mathrm{hx}$. This homomorphic vector is converted into a garbled vector client.gx and its minimum value client.gmin is computed. Finally, $\mathcal{C}$ obtains the intended output by decrypting (converting) client.gmin into the unsigned value client.min.

Type Conversions. Types can be naturally converted into each other by providing them as input to the constructor of the target type, e.g., in Fig. 5.4, the unsigned vector client. $\mathrm{v}$ is converted into the homomorphic vector client.hv via client.hv=HomomorphicVec $(\mathrm{val}=\mathrm{client.v})$. The underlying conversion protocols are described in $\$ 5.1$. 


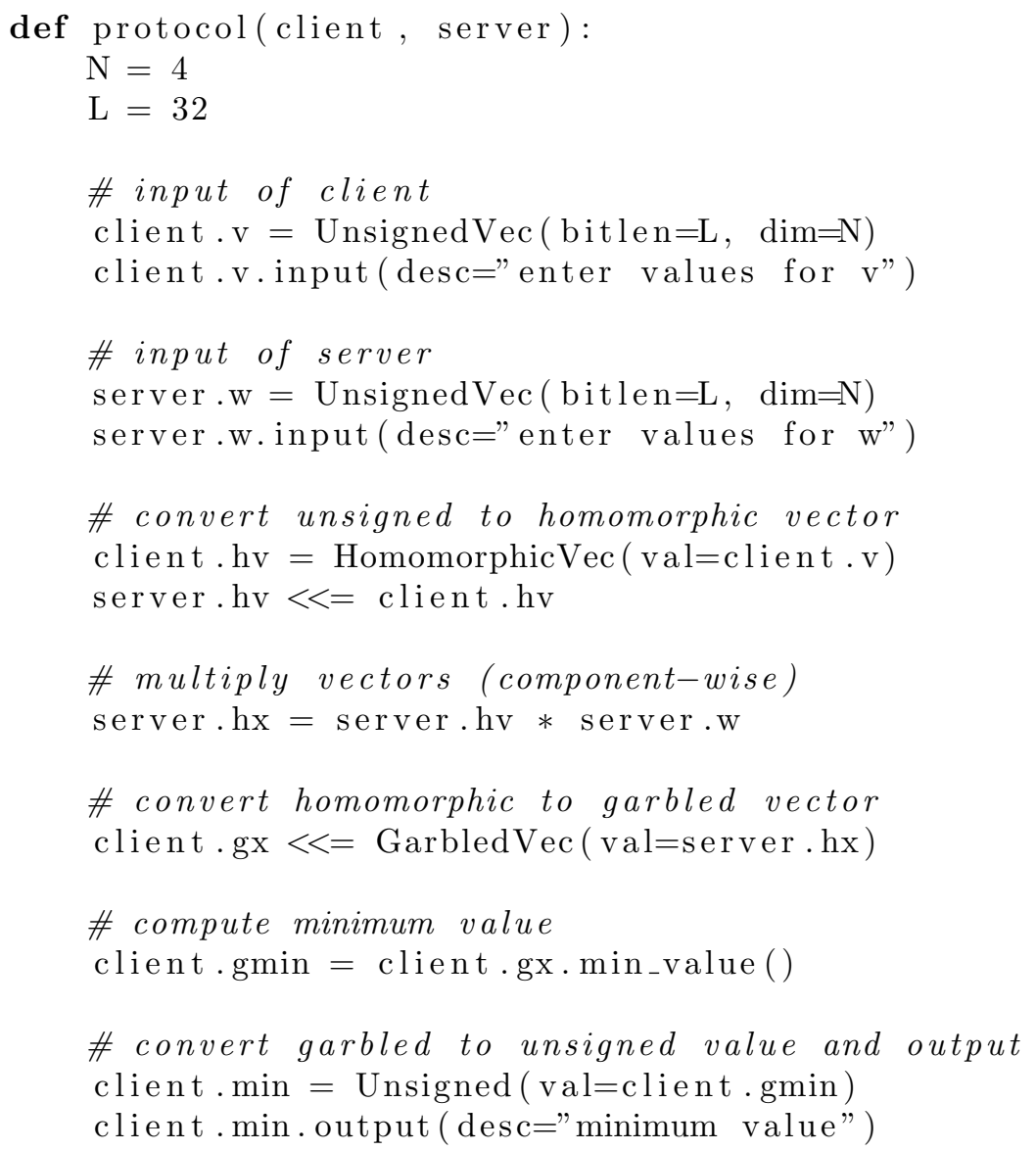

Figure 5.4: TASTYL: Example Program

Send Operator. The send operator $<<=$ transfers variables between the parties, e.g., in Fig. 5.4, hv is sent from $\mathcal{C}$ to $\mathcal{S}$ with server.hv $\ll<=$ client.hv. When combined with a type conversion, the send operator invokes the corresponding conversion protocol, e.g., in Fig. 5.4, homomorphic vector hx held by $\mathcal{S}$ is converted into garbled vector gx held by $\mathcal{C}$ with client.gx $<<=$ GarbledVec(val=server.hx).

Binding of Variables. While constants can be declared globally (e.g., N and L in Fig. (5.4), each variable has to be assigned to one of the parties as an attribute.

Inferring Type and Length Automatically. For each operator, TASTY automatically infers the bit length and type of the output variables from those of the input variables s.t. no overflow occurs. Homomorphic variables raise an exception if the result does not fit into the plaintext space of the homomorphic cryptosystem. For example, in Fig. 5.4 the component-wise product of two vectors with $\mathrm{N}$ components of 
unsigned L-bit values results in the homomorphic vector server. $\mathrm{hx}$ with $\mathrm{N}$ components of unsigned 2L-bit values.

Multiple Outputs. GCs can also have multiple garbled output values written as comma separated list on the left side of the assignment operator, e.g., the garbled minimum value gv and its index gi can be computed as (client.gv, client.gi)=client.gx.min_value_index ().

Circuits from File. TASTY allows secure evaluation of boolean circuits read from an external file, e.g., circuits generated by the FairplayMP compiler [BDNP08]. For this, the labels of the input- and output wires of the circuit are mapped to Garbled Values of corresponding bit length. An example TASTYL file with the concrete syntax for evaluating a garbled file circuit is available at http://tastyproject.net.

\subsubsection{Primitives and Optimizations}

In TASTY we implemented the following efficient primitives and automatic optimizations that allow to move expensive operations as pre-computations into the setup phase (cf. Fig. 5.2) in order to achieve an online phase with low latency. The modular architecture of TASTY allows extension with other primitives as well. In the following we mention the key-features of the used primitives and refer to the previous parts of this thesis and the original papers for details.

Pre-Defined Security Levels. TASTY has pre-defined security levels following standard recommendations of NIST and ECRYPT II [GQ10] as described in \$2.1.1.2. By using matching basic primitives both security and efficiency are optimized simultaneously. We use ECs from the SECG standard [SEC00] and SHA-256 [NIS02] as cryptographic hash function.

Homomorphic Encryption (HE), We use the additively homomorphic cryptosystem of Paillier [Pai99] (cf. \$2.2.1.1). As key generation for Paillier (an RSA modulus $n$ ) is computationally expensive and can be used over multiple protocol runs, the public key is generated and exchanged in the analyzation phase. For efficient encryption we use the extensions of [DJ01, Sect. 6] for pre-computing expensive modular exponentiations of the form $r^{n} \bmod n^{2}$ in the setup phase and only two modular multiplications per encryption in the online phase. As $\mathcal{C}$ knows the factorization $p, q$ of $n$, she uses Chinese remaindering modulo $p$ and $q$ for pre-computing $r^{n} \bmod n^{2}$ and efficient decryption. Paillier ciphertexts have twice the length of the asymmetric security parameter $T$ as the ciphertext space is $\mathbb{Z}_{n^{2}}^{*}$. For modular arithmetics we use gmpy gmpb, a Python wrapper for the GNU Multiple Precision Arithmetic Library (GMP) [gmpa]. 
Garbled Circuit (GC). We use the GC construction with free XORs and garbled row reduction of [PSSW09] secure in the Random Oracle (RO) model (cf. \$2.2.2.3). This GC construction provides free XOR gates (no garbled table and negligible computation). For non-XOR $d$-input gates, the garbled table consists of $2^{d-1}$ entries (of size $t+1$ bit each with symmetric security parameter $t$ ); creation requires $2^{d}$ and evaluation 1 invocation of SHA-256 modeled as RO.

Circuits. For computations on Garbled Values/Vectors, TASTY dynamically generates circuits using the efficient circuit constructions of \$3.3 which are optimized for a low number of non-XOR gates. Alternatively, circuits can be generated externally, e.g., using the Fairplay compiler [MNPS04, BDNP08, and read from a file (cf. §5.2.3.2). TASTY optimizes the circuits to a low number of non-XOR gates using the optimization of $\$ 3.2 .2$ which replaces 3 -input gates with a low number of 2 -input non-XOR gates. XNOR gates are replaced by an XOR gate and an inversion gate which is propagated into successor gates (cf. \$3.2.1.2). Generating, reading, and optimizing circuits is mostly pipelined to allow processing of large circuits with low memory footprint.

Oblivious Transfer (OT), All OTs are pre-computed already in the setup phase (cf. Fig. 5.2) using the construction of [Bea95]; the resulting online phase for OT] is highly efficient (transfer and XOR of bitstrings) and depends mostly on the network latency for two messages (cf. $\$ 2.2 .3 .3$ ). To minimize the computation complexity of the setup phase, we use the efficient OT extension of [IKNP03] to reduce the usually large number of OTs needed in the protocol down to at most $t$ real OTs and some invocations of SHA-256 modeled as $\mathrm{RO}$, where $t$ is the symmetric (computational) security parameter (cf. $\$ 2.2 .3 .2$ ). The remaining real OTs (at most $t$ ) are implemented with the OT protocol of [NP01, Sect. 3.1] using ECs and SHA 256 as RO (cf. \$2.2.3.1). The EC implementation provides point compression to reduce communication at the cost of a negligibly larger computation overhead.

\subsubsection{Performance Measurements}

We have measured the performance of the primitives implemented in TASTY and compared different protocols against each other and with existing SFE implementations: multiplication protocols based on $\mathrm{GC}$ or $\mathrm{HE}$ (5.2.5.1) and SFE of an AES circuit generated by the Fairplay compiler (\$5.2.5.2).

System Setup. All performance measurements were performed on two desktop PCs with Intel Core 2 Duo CPU (E6850) running at $3.0 \mathrm{GHz}$ and $4 \mathrm{~GB}$ Random Access Memory (RAM) connected via Gigabit Ethernet. The system runs on 64bit Gentoo Linux with Python version 2.6.5, gmpy version 1.11 gmpb and GMP version 4.3.2 
[gmpa]. Unless stated otherwise, all measurements were performed for short-term security (cf. Table 2.2 in $\$ 2.1 .1 .2$ ) and using point compression for ECs (cf. \$5.2.4).

\subsubsection{Multiplication Protocols}

As arithmetic circuits can express arbitrary computations as sequence of additions and multiplications (cf. \$2.1.3.2), multiplication is a fundamental basic operation. Indeed, the main difference between SFE protocols based on arithmetic and boolean circuits is the cost for multiplications.

Using TASTY we compare the performance of different secure multiplication protocols based on $\mathrm{HE}$ and GCs using fast multiplication circuits (cf. \$3.3.2). For this we constructed four basic test cases. For each SFE paradigm we consider the case where both inputs are provided by one party $(\mathcal{S}$ for GC1 and $\mathcal{C}$ for HE1), or one by each of the parties (GC2 and HE2). The inputs are Unsigned $\ell$-bit values and the output, a $2 \ell$-bit Unsigned value, is converted into a Plain output for $\mathcal{C}$. In the following, we compare the communication- and the computation complexity of the setup- and online phase of the protocols.

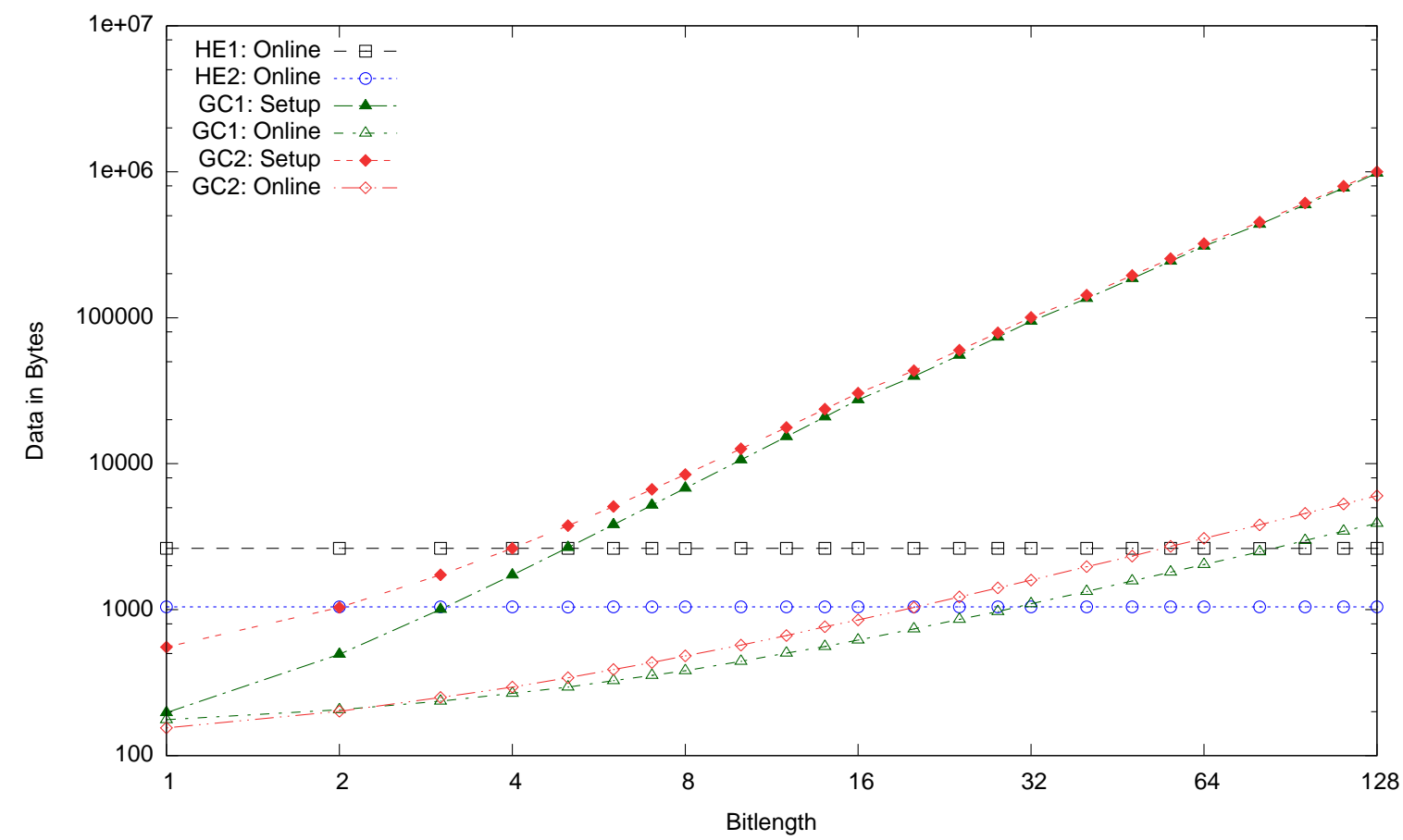

Figure 5.5: Secure Multiplication Protocols: Communication 
Communication (cf. Fig. 5.5). Our experiments show that GC-based multiplication requires a substantial amount of setup communication (for transfer of GCs) whereas the online communication of $\mathrm{GC}$ is better than $\mathrm{HE}$ for multiplication of small values. The online communication for multiplying with $\overline{\mathrm{HE}}$ is independent of the bit length $\ell$ as a constant number of ciphertexts (2 for HE1 and 5 for HE2) is exchanged. For multiplying with $\mathrm{GC}$, the setup communication grows rapidly due to the large size of the GCs, whereas the online communication complexity grows much slower.

Setup Time (cf. Fig. $\mathbf{5 . 6 ( a )}$ ). The time of the setup phase for GC-based multiplication protocols depends on the bit length $\ell$ as GCs need to be computed; for better visualization we do not plot GC setup times for $\mathcal{S}$ in Fig. 5.6(a) as they are similar to those of $\mathcal{C}$ (in our current implementation, $\mathcal{C}$ waits until $\mathcal{S}$ has created the GC). For HE-based multiplication, the setup time is independent of $\ell$ as a constant number of encryptions is pre-computed.

Online Time (cf. Fig. 5.6(b)). For GC-based multiplication, the time needed by $\mathcal{C}$ depends on the size of the evaluated $\mathrm{GC}$ which grows with the bit length $\ell$; GC]s online time for $\mathcal{S}$ is negligible. For HE based multiplication, the time in the online phase is almost independent of $\ell$ for small bit lengths.

Conclusion. The setup phase for GC-based multiplication is substantially more expensive than that of HE-based multiplication. However, for small values, GC-based multiplication can result in a faster online time than HE-based multiplication. Furthermore, GC-based multiplication, in contrast to HE-based multiplication, needs no (when composed with other GC-based computations) or negligible online interaction and workload for $\mathcal{S}$.

Parallel Multiplications. When $N$ multiplications are done in parallel, e.g., component-wise multiplication of two vectors of $N$ components, time and data complexity of GC-based multiplication grows linearly in $N$. HE-based parallel multiplication increases slower as multiple homomorphic values can be packed before sending from $\mathcal{S}$ to $\mathcal{C}$ (cf. 2.2 .1 .3 ).

Dependence on Security Level. We note that when the security level is increased to medium- or even long-term security, the performance of HE-based multiplication decreases rapidly while the performance of GC-based multiplication is affected only moderately, as the asymmetric security parameter grows substantially faster than the symmetric one (cf. Table 2.2 in 2.1.1.2). 


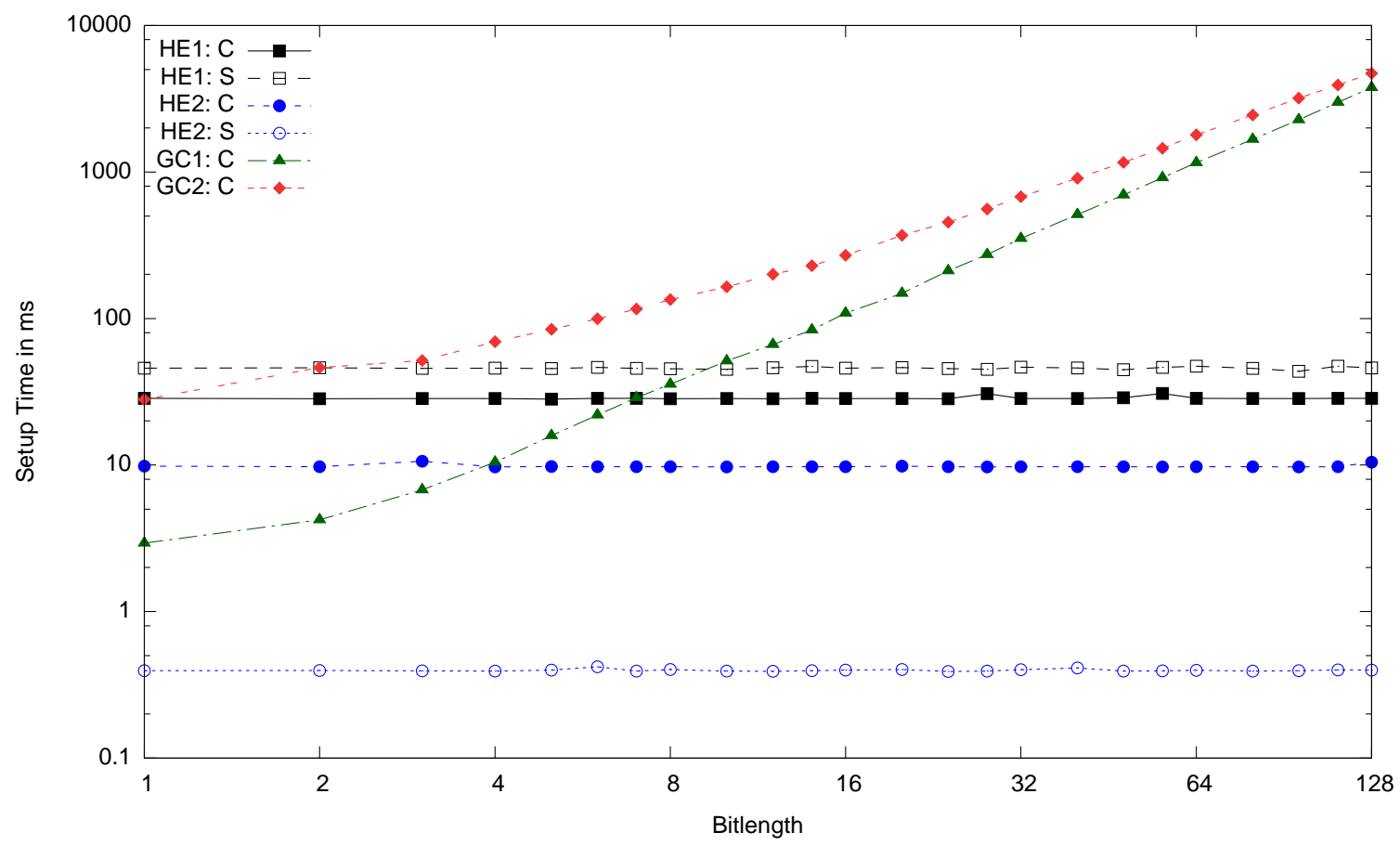

(a) Setup Time

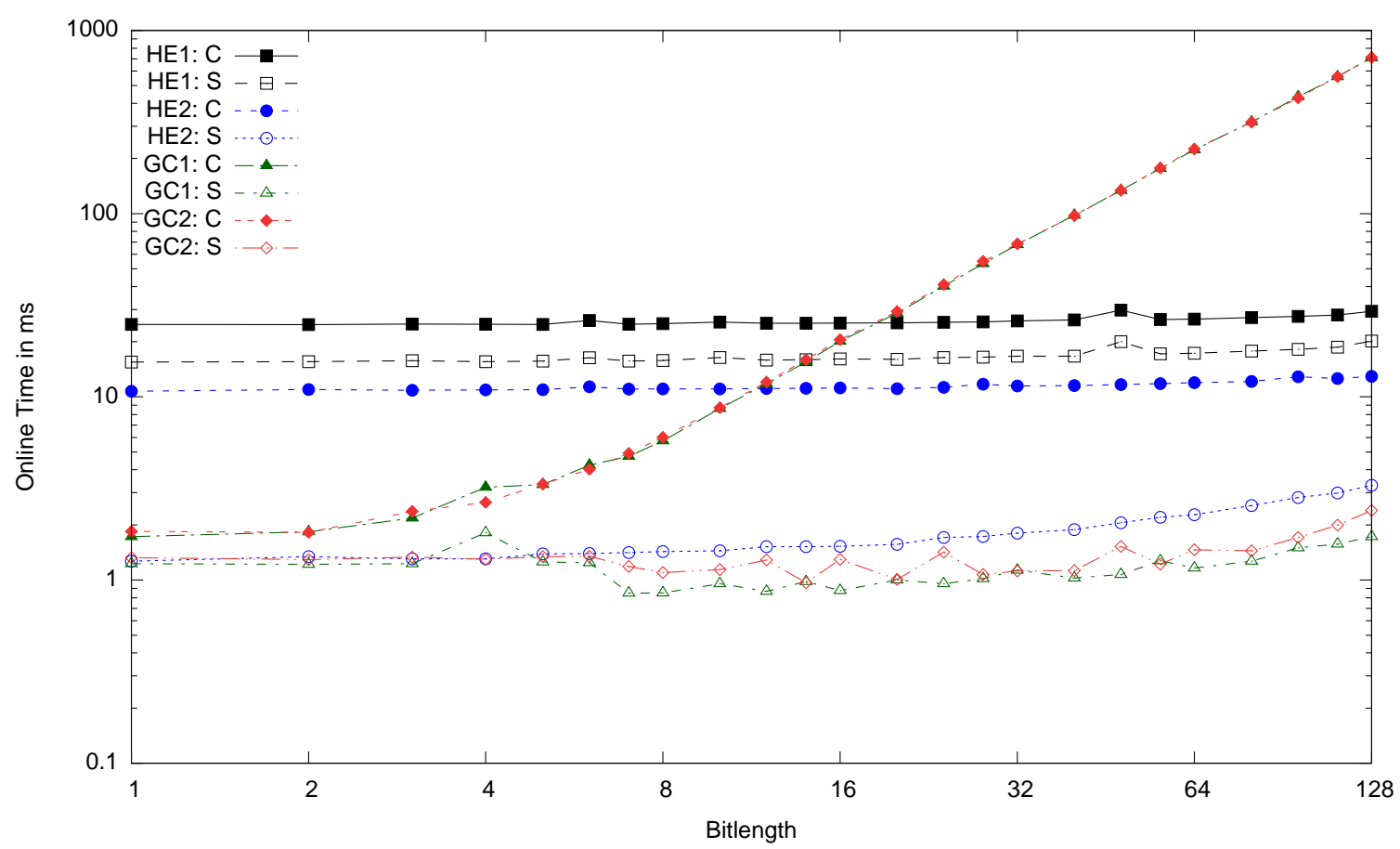

(b) Online Time

Figure 5.6: Secure Multiplication Protocols: Timings 


\subsubsection{Evaluation of Fairplay Circuits and AES}

As described in $\$ 5.2 .3 .2$, TASTY can evaluate circuits generated by the FairplayMP compiler BDNP08. Using this feature, we compare the performance of TASTY for evaluation of the AES functionality with the state of the art software implementation of GCs reported in [PSSW09, Table 2] which is implemented in $\mathrm{C}++$ and measured on two machines also with Intel Core 2 Duo's running at $3.0 \mathrm{GHz}$ and $4 \mathrm{~GB}$ of RAM connected by gigabit ethernet. We use the AES circuit of [PSSW09] which has 128 input bits provided by each party, 128 output bits for $\mathcal{C}$ and is optimized for a low number of non-XOR gates (22,594 XOR gates and 11, 286 non-XOR 2-input gates).

Table 5.2: Performance Comparison: GC Evaluation of AES (times in seconds)

\begin{tabular}{c|c||c|c|c||c|}
\cline { 4 - 6 } \multicolumn{1}{c|}{} & \multicolumn{3}{c||}{ Time } & KByte \\
\cline { 2 - 5 } \multicolumn{1}{c|}{} & Security & Setup & Online & Total & Total \\
\hline \hline [MNPS04 & ultra-short & - & - & $\mathbf{4}$ & 3760 \\
\hline TASTY & ultra-short & 2.9 & $\mathbf{0 . 4}$ & 3.3 & 567 \\
\hline \hline TSSW09 & long & 2 & $\mathbf{5}$ & 7 & 503 \\
\hline TASTY & long & 4.0 & $\mathbf{0 . 5}$ & 4.5 & 860 \\
\hline
\end{tabular}

The performance of different GC implementations for evaluating the AES functionality is compared in Table 5.2 .

For ultra-short-term security (cf. Table 2.2 in 82.1 .1 .2 ), when evaluating AES with Fairplay's Java runtime [MNPS04], we see that Fairplay requires substantially more communication than TASTY, as Fairplay provides no free XOR gates $(2 / 3$ of the gates are XOR gates). Also TASTY s time complexity is slightly better than that of Fairplay due to free XOR and more efficient OT over ECs.

Also for long-term security (cf. Table 2.2 in $\$ 2.1 .1 .2$ ), TASTY s online phase is faster than that of [PSSW09] by an order of magnitude. Recall, a short online phase, i.e., latency from providing the inputs until obtaining the outputs, is important for many real-world applications. To minimize this, TASTY] shifts most computations into the less time-critical setup phase (cf. 55.2 .2 ). Also TASTY has a slightly shorter total time than [PSSW09], whereas the data complexity is slightly larger due to less optimal data serialization in our implementation. More detailed, the setup time of [PSSW09] is $1 \mathrm{~s}$ for $\mathrm{GC}$ creation and $1 \mathrm{~s}$ for data transfer, and the online time is $3 \mathrm{~s}$ for OT and $2 \mathrm{~s}$ for $\mathrm{GC}$ evaluation. In TASTY, the setup time is dominated by $1.1 \mathrm{~s}$ for $\mathrm{OT}$ and $1.8 \mathrm{~s}$ for $\mathrm{GC}$ creation, and the online time is dominated by $0.4 \mathrm{~s}$ for $\mathrm{GC}$ evaluation.

\footnotetext{
${ }^{5} \mathrm{As}$ OT seemed not to be the performance bottleneck in PSSW09, this implementation used a less
} efficient, universally composable $\mathrm{OT}$ protocol also in the semi-honest setting. 


\subsection{Application: Privacy-Preserving Face Recognition}

Automatic recognition of human faces is becoming increasingly popular in civilian and law enforcement applications that require reliable recognition of humans. However, the rapid improvement and widespread deployment of this technology raises strong concerns regarding the violation of individuals' privacy. A typical application scenario for privacy-preserving face recognition concerns a client who privately searches for a specific face image in the face image database of a server.

In this section we present protocols for privacy-preserving face recognition based on the Eigenfaces algorithm that substantially improve over previous works that are based only on Homomorphic Encryption (HE) in terms of efficiency and security: the protocol proposed in $\left[\mathrm{EFG}^{+} 09\right](\$ 5.3 .3 .2)$ requires $\mathcal{O}(\log M)$ rounds to recognize a face in a database of $M$ faces, whereas the proposal in [BBC $\left.{ }^{+} 10\right]$ ( $\$ 5.3 .3 .4$ achieves only a weaker definition of security where the client is allowed to learn intermediate information.

Our protocols require only $\mathcal{O}(1)$ rounds and allow to shift most computations into a setup phase. The protocols are based on a combination of HE and Garbled Circuits $(\mathrm{GCs})$ in the modular Secure Function Evaluation (SFE) framework of \$5.1. Alternatively, our protocols can also be used for more efficient and secure privacy-preserving fingercode authentication.

Publication Info: Parts of the following results are published in [SSW09] and $\left[\mathrm{HKS}^{+} 10\right]$.

\subsubsection{Motivation}

In the last decade biometric identification and authentication have increasingly gained importance for a variety of enterprise, civilian and law enforcement applications. Examples vary from fingerprinting and iris scanning systems, to voice and face recognition systems, etc. Many governments have already rolled out electronic passports [nt03] and IDs [NH08] that contain biometric information (e.g., image, fingerprints, and iris scan) of their legitimate holders.

In particular it seems that facial recognition systems have become popular aimed to be installed in surveillance of public places Gro08, and access and border control at airports [Bow] to name some. For some of these use cases one requires online search with short response times and low amount of online communication.

Moreover, face recognition is ubiquitously used also in online photo albums such as Google Picasa and social networking platforms such as Facebook which have become popular to share photos with family and friends. These platforms support automatic 
detection and tagging of faces in uploaded images ${ }^{6}$ Additionally, images can be tagged with the place they were taken. 7

The widespread use of such face recognition systems, however, raises also privacy risks since biometric information can be collected and misused to profile and track individuals against their will. These issues raise the desire to construct privacy-preserving face recognition systems $\left.\left[\mathrm{EFG}^{+} 09\right]\right]^{8}$

We concentrate on efficient privacy-preserving face recognition systems. The typical scenario here is a client-server application where the client needs to know whether a specific face image is contained in the database of a server with the following requirements: the client trusts the server to correctly perform the matching algorithm for the face recognition but without revealing any useful information to the server about the requested image as well as about the outcome of the matching algorithm. The server requires privacy of its database beyond the outcome of the matching algorithm to the client. In $\left[\mathrm{BBC}^{+} 10\right]$ it was shown that similar techniques can be used for privacy-preserving fingercode authentication, where a fingerprint is matched against a database of fingerprints (cf. \$5.3.3.4).

In the proposal for privacy-preserving face recognition of [EFG ${ }^{+}$09] (cf. \$5.3.3.2 for details) the authors use the standard and popular Eigenface [TP91b, TP91a] recognition algorithm and design a protocol that performs operations on encrypted images by means of $\mathrm{HE}$ only. They demonstrate that privacy-preserving face recognition is possible in principle and give required choices of parameter sizes to achieve a good classification rate. However, the proposed protocol requires $\mathcal{O}(\log M)$ rounds of online communication as well as computationally expensive operations on homomorphically encrypted data to recognize a face in the database of $M$ faces. Due to these restrictions, the proposed protocol cannot be deployed in practical large-scale applications. We address this aspect and show that one can do better w.r.t. efficiency.

The subsequent proposal of [ $\left.\mathrm{BBC}^{+} 10\right]$ (details in $\$ 5.3 .3 .4$ ) is also based on $\mathrm{HE}$ only and improves efficiency to $\mathcal{O}(1)$ rounds at the cost of the client learning additional information.

Our Contribution. We give an efficient and secure privacy-preserving face recognition protocol based on the Eigenfaces recognition algorithm [TP91b, TP91a and a hybrid SFE protocol (using the modular SFE framework of \$5.1) which combines the advantages of $\mathrm{HE}$ (low communication for linear operations) and GCs (constant round complexity for minimum search). Compared to previous protocols of $\left[\mathrm{EFG}^{+} 09\right.$, $\left.\mathrm{BBC}^{+} 10\right]$, our protocol has only a constant number of $\mathcal{O}(1)$ rounds and allows to shift most of the computation and communication into a pre-computation phase. The re-

\footnotetext{
${ }^{6}$ http://picasa.google.com/features-nametags.html; http://face.com

${ }^{7}$ Geotagging can be done either manually or automatically on iPhones using GPS http://www. saltpepper.net/geotag

${ }^{8}$ Similar concerns motivated previous research directions on privacy-preserving iris scanning $\mathrm{BDK}^{+} 05$, or fingerprinting $\mathrm{TAK}^{+} 05$.
} 
maining online phase is highly efficient and allows for a quick response time which is especially important in applications such as biometric access control.

Related Work. Privacy-Preserving Face Recognition allows a client to obliviously detect if the image of a face is contained in a database of faces held by server. We give a detailed summary of previous works on privacy-preserving face recognition based on the Eigenface recognition algorithm in $\$ 5.3 .3 .2$ for $\left[\mathrm{EFG}^{+} 09\right]$ and $\$ 5.3 .3 .4$ for $\left[\mathrm{BBC}^{+} 10\right]$.

The subsequent work of [HMEK11] considers the simplified setting where parts of the recognition algorithm, namely the Projection phase (cf. \$5.3.2), are not performed securely. As discussed, in [EFG+09], this setting might be justified in some scenarios where the Eigenfaces are computed from a (sufficiently large) public database of faces, or the server is willing to reveal this information to the client.

SCiFI, a recently proposed system for secure face identification OPJM10 combines a novel recognition algorithm with a co-designed highly efficient special-purpose SFE protocol. As described in OPJM10, this combination is more accurate and robust (tolerates environmental conditions such as light or persons wearing glasses) than Eigenface-based protocols.

The related problem of Privacy-Preserving Face Detection [AB06] allows a client to detect faces on her image using a private classifier held by server without revealing the face or the classifier to the other party.

In order to preserve privacy, faces can be de-identified such that face recognition software cannot reliably recognize de-identified faces, even though many facial details are preserved as described in [NSM05.

\subsubsection{Face Recognition using Eigenfaces}

A well-known algorithm for face recognition is the so-called Eigenfaces algorithm introduced in [TP91b, TP91a]. This algorithm achieves reasonable classification rates of approximately $96 \%\left[\mathrm{EFG}^{+} 09\right]$ and can be implemented as privacy-preserving protocol (cf. \$5.3.3). The Eigenfaces algorithm transforms face images into their characteristic feature vectors in a low-dimensional vector space (face space), whose basis consists of Eigenfaces. The Eigenfaces are determined through Principal Component Analysis (PCA) from a set of training images; every face is represented as a vector in the face space by projecting the face image onto the subspace spanned by the Eigenfaces. Recognition is done by first projecting the face image into the face space and afterwards locating the closest feature vector. For details on the enrollment process we refer to [EFG ${ }^{+}$09] and original papers on Eigenfaces [TP91b, TP91a]. In the following we briefly summarize the recognition process of the Eigenfaces algorithm. A pseudocode description and the naming conventions is given in Algorithm 3; the naming conventions and parameter sizes determined in $\left[\mathrm{EFG}^{+} 09\right]$ are listed in Table 5.3. 
Inputs and Outputs: The algorithm obtains as input the query face image $\Gamma$ represented as a pixel image with $N$ pixels. Additionally, the algorithm obtains the parameters determined in the enrollment phase as inputs: the average face $\Psi$ which is the mean of all training images, the Eigenfaces $u_{1}, . ., u_{K}$ which span the $K$-dimensional face space, the projected faces $\Omega_{1}, . ., \Omega_{M}$ being the projections of the $M$ faces in the database into the face space, and the threshold value $\tau$. The output $r$ of the recognition algorithm is the index of that face in the database which is closest to the query face $\Gamma$ or the special symbol $\perp$ if no match was found, i.e., all faces have a larger distance than the threshold $\tau$.

Recognition Algorithm: The recognition algorithm consists of three phases:

1. Projection: First, the average face $\Psi$ is subtracted from the face $\Gamma$ and the result is projected into the $K$-dimensional face space using the Eigenfaces $u_{1}, . ., u_{K}$. The result is the projected $K$-dimensional face $\bar{\Omega}$.

2. Distance: Afterwards, the square of the Euclidean distance $D_{i}$ between the projected $K$-dimensional face $\bar{\Omega}$ and all projected $K$-dimensional faces in the database $\Omega_{i}, i=1, . ., M$, is computed.

3. Minimum: Finally, the minimum distance $D_{\min }$ is selected. If $D_{\min }$ is smaller than threshold $\tau$, the index of the minimum value, i.e., the identifier $i_{\min }$ of the match found, is returned to $\mathcal{C}$ as result $r=i_{\text {min }}$. Otherwise, the image was not found and the special symbol $r=\perp$ is returned.

\begin{tabular}{|r|l|l|}
\hline Parameter & Size $\left[\mathrm{EFG}^{+} 09\right]$ & Description \\
\hline$M$ & $=10304$ & number of faces in database \\
$K$ & $=12$ & size of a face in pixels \\
$\Gamma, \Psi$ & $\in\left[0,2^{8}-1\right]^{N}$ & number of Eigenfaces \\
face, average face \\
$u_{1}, . ., u_{K}$ & $\in\left[-2^{7}, 2^{7}-1\right]^{N}$ & Eigenfaces \\
$\bar{\Omega}, \Omega_{1}, . ., \Omega_{M}$ & $\in\left[-2^{31}, 2^{31}-1\right]^{K}$ & projected face, projected faces in database \\
$D_{1}, . ., D_{M}$ & $\in\left[0,2^{50}-1\right]$ & squared distances between projected images \\
$\tau$ & $\in\left[0,2^{50}-1\right]$ & threshold value \\
\hline
\end{tabular}

Table 5.3: Parameters and Sizes: Privacy-Preserving Face Recognition

\subsubsection{Privacy-Preserving Face Recognition}

Privacy-Preserving Face Recognition allows a client to obliviously detect if the image of a face is contained in a database of faces held by a server. This can be achieved by 


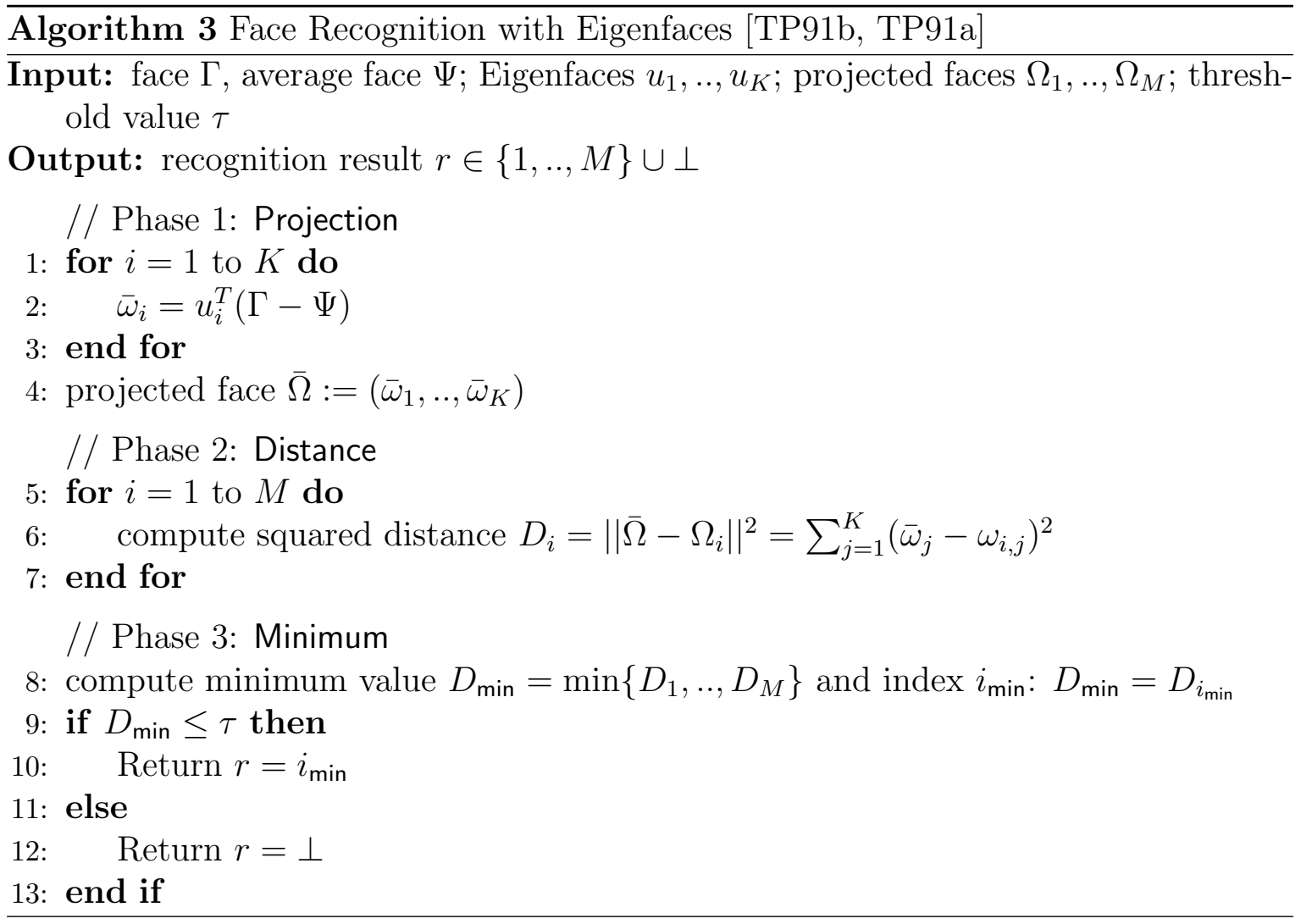

securely evaluating a face recognition algorithm within a cryptographic protocol. In the following we concentrate on the Eigenface algorithm described in $\$ 5.3 .2$ which was also used in $\left[\mathrm{EFG}^{+} 09\right]$.

\subsubsection{Privacy-Preserving Face Recognition using Eigenfaces}

The inputs and outputs of the Eigenfaces algorithm are distributed between client $\mathcal{C}$ and server $\mathcal{S}$ as shown in Fig. 5.7. Both parties want to hide their inputs from the other party during the protocol run, i.e., $\mathcal{C}$ does not want to reveal for which face she is searching while $\mathcal{S}$ does not want to reveal the faces in his database or the details of the applied transformation into the face space (including Eigenfaces which might reveal critical information about faces in DB).

In the semi-honest model we are working in (cf. \$2.1.4), parties are assumed to follow the protocol but try to learn additional information from the protocol trace beyond what can be derived from the inputs and outputs of the algorithm when used as a black-box. In particular this requires that all internal results of the Eigenfaces algorithm, including the values passed between the different phases $\bar{\Omega}$ and $D_{1}, . ., D_{M}$, are "hidden" from both parties. For practical applications it is sufficient to assume that both parties are computationally bounded, i.e., no polynomial-time adversary can 
derive information from "hidden" values.

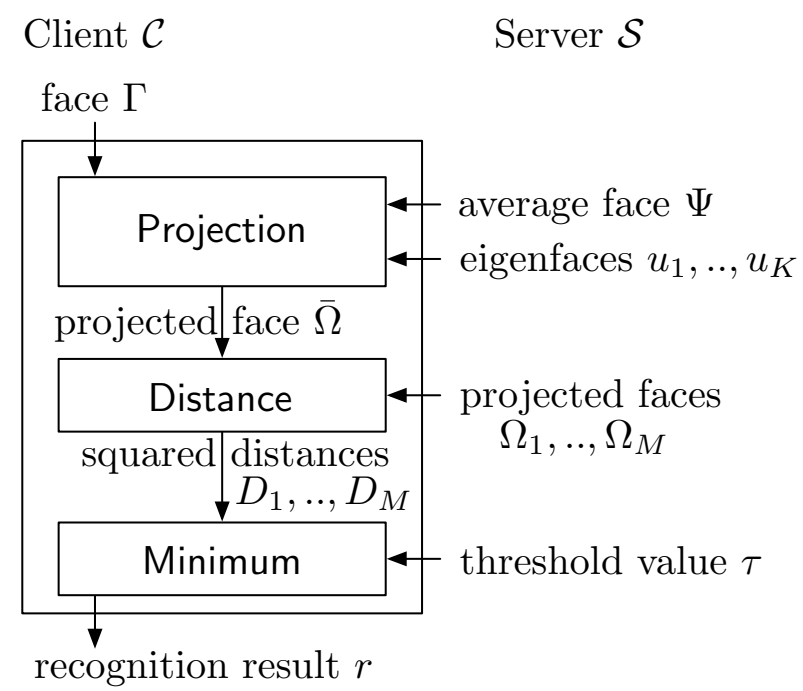

Figure 5.7: Protocol Structure: Secure Face Recognition using Eigenfaces

In the following we present the initial proposal of [EFG $\left.{ }^{+} 09\right]$ for implementing the privacy-preserving Eigenfaces algorithm and "hiding" the intermediate values.

\subsubsection{Initial Protocol of $\mathrm{EFG}^{+} 09$}

In $\mathrm{EFG}^{+}$09], the authors describe a protocol for privacy-preserving face recognition which implements the Eigenfaces recognition algorithm of $\$ 5.3 .2$ using HE. Their protocol is secure in the semi-honest model, i.e., players are honest-but-curious $\mathrm{EFG}^{+} 09$, Appendix A].

Projection. First, $\mathcal{C}$ and $\mathcal{S}$ jointly compute the projection of the face image $\Gamma$ into the Eigenspace spanned by the Eigenfaces $u_{1}, . ., u_{K}$ as follows: $\mathcal{C}$ generates a secret/public key pair of a HE scheme (cf. \$2.2.1.1) and encrypts the face $\Gamma$ as $\llbracket \Gamma \rrbracket=\left(\llbracket \Gamma_{1} \rrbracket, . ., \llbracket \Gamma_{N} \rrbracket\right)$. $\mathcal{C}$ sends the encrypted face $\llbracket \Gamma \rrbracket$ along with the public key to $\mathcal{S}$. Using the homomorphic properties, $\mathcal{S}$ projects the encrypted face into the low-dimensional face space and obtains the encryption of the projected face $\llbracket \bar{\Omega} \rrbracket=\left(\llbracket \bar{\omega}_{1} \rrbracket, . ., \llbracket \bar{\omega}_{K} \rrbracket\right)$ by computing for $i=1, . ., K: \llbracket \bar{\omega}_{i} \rrbracket=\llbracket-\sum_{j=1}^{N} u_{i, j} \Psi_{j} \rrbracket \boxplus \sum_{j=1}^{N} u_{i, j} \llbracket \Gamma_{j} \rrbracket$. The first factor can already be computed in the pre-computation phase.

Performance Improvement. Additionally we observe that the values $\llbracket \bar{\omega}_{i} \rrbracket$ can be accumulated in parallel by using a parallel fast exponentiation algorithm which reuses the same squared values of $\llbracket \Gamma_{j} \rrbracket$ in the square-and-multiply method. 
Distance. After Projection, $\mathcal{C}$ and $\mathcal{S}$ jointly compute the encryption of the Euclidean distances between the projected face $\llbracket \bar{\Omega} \rrbracket$ and all projected faces $\Omega_{1}, . ., \Omega_{M}$ in the database held by $\mathcal{S}$. This is done by computing for $i=1, . ., M$ : $\llbracket D_{i} \rrbracket=$ $\llbracket|| \Omega_{i}-\bar{\Omega} \|^{2} \rrbracket=\llbracket S_{1, i} \rrbracket \cdot \llbracket S_{2, i} \rrbracket \cdot \llbracket S_{3} \rrbracket$, where $\llbracket S_{1, i} \rrbracket=\llbracket \sum_{j=1}^{K} \omega_{i, j}^{2} \rrbracket=\sum_{j=1}^{K} \llbracket \omega_{i, j}^{2} \rrbracket$ and $\llbracket S_{2, i} \rrbracket=\llbracket \sum_{j=1}^{K}\left(-2 \omega_{i, j} \bar{\omega}_{j}\right) \rrbracket=\sum_{j=1}^{K}-2 \omega_{i, j} \llbracket \bar{\omega}_{j} \rrbracket$ can be computed by $\mathcal{S}$ from $\llbracket \bar{\Omega} \rrbracket$ without interaction with $\mathcal{C}$.

We note that the values $\llbracket S_{1, i} \rrbracket$ can be pre-computed entirely and online computation of $\llbracket S_{2, i} \rrbracket$ can be speeded up by accumulating these values in parallel in order to re-use the same squares in the square-and-multiply exponentiation algorithm.

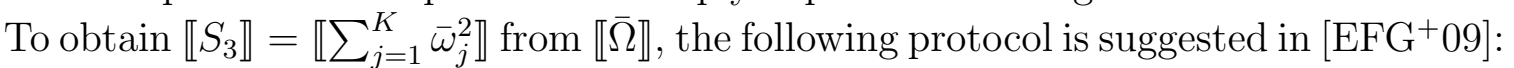
For $j=1, . ., K: \mathcal{S}$ chooses $r_{j} \in_{R} \mathbb{Z}_{n}$, computes $\llbracket x_{j} \rrbracket=\llbracket \bar{\omega}_{j}+r_{j} \rrbracket=\llbracket \bar{\omega}_{j} \rrbracket \boxplus \llbracket r_{j} \rrbracket$ and sends $\llbracket x_{j} \rrbracket$ to $\mathcal{C}$. $\mathcal{C}$ decrypts $\llbracket x_{j} \rrbracket$, computes $\llbracket S_{3}^{\prime} \rrbracket=\llbracket \sum_{j=1}^{K} x_{j}^{2} \rrbracket$, and sends $\llbracket S_{3}^{\prime} \rrbracket$ to $\mathcal{S}$. $\mathcal{S}$ finally computes $\llbracket S_{3} \rrbracket=\llbracket S_{3}^{\prime} \rrbracket \boxplus \llbracket-\sum_{j=1}^{K} r_{j}^{2} \rrbracket \boxplus \sum_{j=1}^{K}-2 r_{j} \llbracket \bar{\omega}_{j} \rrbracket$.

Performance Improvement. As proposed in [SSW09, Full Version, Appendix C], $\llbracket S_{3} \rrbracket$ can be computed more efficiently by choosing shorter random masks and using packing, similar to the parallel multiplication protocol of $\$ 2.2 .1 .3$.

Minimum. Finally, $\mathcal{C}$ and $\mathcal{S}$ jointly compute the minimum value $D$ from $\llbracket D_{1} \rrbracket, . ., \llbracket D_{M} \rrbracket$ and its index Id. If the minimum value $D$ is smaller than or equal to the threshold value $\tau$ known by $\mathcal{S}$, then $\mathcal{C}$ obtains the result Id. To achieve this, EFG $\mathrm{EF}^{+}$suggests the following protocol: Choose the minimum value and index from the list of encrypted value and id pairs $\left(\llbracket D_{0}=\tau \rrbracket, \llbracket \operatorname{ld}_{0}=\perp \rrbracket\right),\left(\llbracket D_{i} \rrbracket, \llbracket \operatorname{ld}_{i} \rrbracket\right)_{i=1}^{M}$. For this, they apply a straight-forward recursive algorithm for minimum selection based on a subprotocol which compares two encrypted distances and returns a re-randomized encryption of the minimum and its index to $\mathcal{S}$. For this sub-protocol, they adapt the comparison protocol of [BK04] using the Damgård, Geisler and Krøigaard (DGK) [DGK07, DGK08a, DGK08b] cryptosystem (cf. \$3.4.1).

Complexity of Minimum protocol of $\left[\mathbf{E F G}^{+} \mathbf{0 9}\right]$. The Minimum protocol proposed in [EFG $\left.{ }^{+} 09\right]$ requires a logarithmic number of $6\left\lceil\log _{2}(M+1)\right\rceil+1$ moves. Overall, $8 M$ Paillier ciphertexts (of size $2 T$ bits each) and $2 \ell^{\prime} M$ DGK ciphertexts (of size $T$ bits each) are sent in the online phase, where $\ell^{\prime}=50$ is the length of the squared distances $D_{1}, . ., D_{M}$ among which the minimum is selected (cf. Table 5.3). This results in a communication complexity of $\left(16+2 \ell^{\prime}\right) M T$ bits. The asymptotic online computation complexity is dominated by approximately $2 M$ Paillier decryptions and $\ell^{\prime} M$ DGK decryptions for $\mathcal{C}$ and the same number of exponentiations for $\mathcal{S}$.

As shown in 3.4 .1 , in the two-party case, there exist protocols for computing the minimum of two values that are substantially more efficient than the DGK protocol. 
These are the key for computing the Minimum step more efficiently and hence improving privacy-preserving face recognition as described in the following.

\subsubsection{Improved Minimum Protocol of [SSW09]}

A first approach is to construct a hybrid protocol using the modular SFE framework of $§ 5.1$, where the Minimum step is computed more efficiently using a GCs: For this, the vector of $\mathrm{HEs} \llbracket D_{1} \rrbracket, . ., \llbracket D_{M} \rrbracket$ is first converted into its garbled equivalent $\widetilde{D}_{1}, . ., \widetilde{D}_{M}$ using the conversion protocol of $\$ 5.1 .3 .2$. Then, a $\mathrm{GC}$ is evaluated that computes the minimum value and index as described in 3.3 .3 .3 . Finally, the resulting minimum distance is compared against the threshold value and depending on this, either $\perp$ or the minimum index is output to $\mathcal{C}$.

This approach immediately yields constant round complexity and allows to shift most computations into the setup phase. The resulting complexity of the online phase is substantially more efficient than the protocol of [EFG+ 09] as verified theoretically and experimentally in [SSW09]. Using TASTY, the protocol can be generated automatically from the TASTY input Language (TASTYL) code shown in Fig. 5.8 $\left[\mathrm{HKS}^{+} 10\right]$.

\subsubsection{Alternative Minimum Protocol of $\left[\mathrm{BBC}^{+} 10\right]$}

The techniques for privacy-preserving face recognition can also be adapted for privacypreserving fingerprint matching as proposed in $\left[\mathrm{BBC}^{+} 10\right]$. In their alternative application scenario, the minimum phase needs to return all the identifiers whose distance is less than a given threshold. Clearly, this is easier to achieve than in the case of privacy-preserving face recognition where this information needs to be hidden from $\mathcal{C}$.

The minimum protocol proposed in $\left[\mathrm{BBC}^{+} 10\right]$ is similar to that of $\left[\mathrm{EFG}^{+} 09\right]$ with the following modifications. For better efficiency they use EC-ElGamal instead of DGK encryption (cf. $\$ 3.4 .1$ ). To reduce the round complexity from logarithmic to constant, they compare each of the homomorphically encrypted distances $\llbracket D_{i} \rrbracket$ with the threshold value in parallel. We will extend on this idea in our more improved hybrid protocol described in $\$ 5.3 .4$.

Remarks. The authors of $\left[\mathrm{BBC}^{+} 10\right]$ claim that, in comparison with the Minimum protocols of [EFG+ 09, SSW09], their protocol described in $\left[\mathrm{BBC}^{+} 10\right.$, Sect. 4.4.1] is

1. functionally equivalent $\left[\mathrm{BBC}^{+} 10\right.$, Sect. 4.4.1], and

2. notably more communication efficient $\left[\overline{\mathrm{BBC}^{+}} 10\right.$, Sect. 4.5$] .9$

\footnotetext{
${ }^{9}$ The communication efficiency of the protocol of $\left[\mathrm{BBC}^{+} 10\right.$, Sect. 4.4 .1$]$ is exactly the same as that
} of $\left[\mathrm{BBC}^{+} 10\right.$, Fig. 2]. 


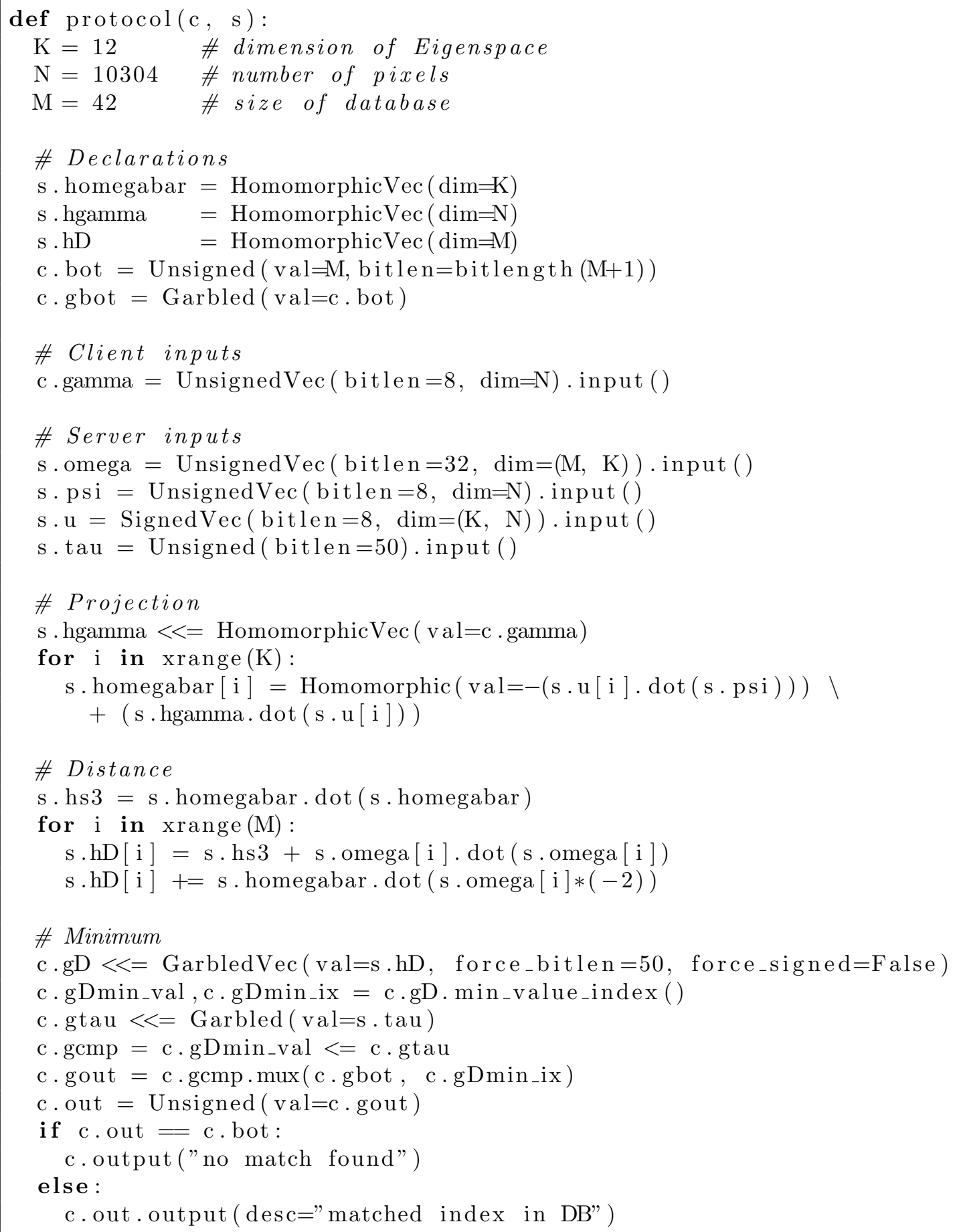

Figure 5.8: TASTYL, Improved Privacy-Preserving Face Recognition of [SSW09] 
With respect to functional equivalency we observe that, in contrast to the protocols of [EFG ${ }^{+}$09, SSW09], in the protocol of $\left[\mathrm{BBC}^{+} 10\right.$, Sect. 4.4.1] the client $\mathcal{C}$ obtains as additional information how many of the entries in the database match the query, i.e., $\# D_{i}<\tau: 10$ It depends on the application scenario, whether this additional information can be tolerated or not.

With respect to efficiency we will show in $\$ 5.3 .4$ how the protocol of [SSW10] can be slightly changed such that, even when pre-computations are not possible, the protocol is slightly more communication efficient than the protocol of $\left[\mathrm{BBC}^{+} 10\right.$, Sect. 4.4.1] for the fingerprint matching scenario, while enjoying the additional security property that $\mathcal{C}$ does not learn the cardinality of matching entries.

Complexity of Minimum protocol of $\left[\mathrm{BBC}^{+} 10\right.$, Sect. 4.4.1]. The Minimum protocol of $\left[\mathrm{BBC}^{+} 10\right.$, Sect. 4.4.1] requires a constant number of 5 moves. Using packing, $2 M+m^{\prime}$ Paillier ciphertexts (of size $2 T$ bits each) and $2 M \ell^{\prime}$ EC-ElGamal ciphertexts (of size 2(2t+1) bits each using point compression) are sent in the online phase, where $m$ Paillier ciphertexts are needed to transfer $M$ packed $\left(\sigma+\ell^{\prime}+1\right)$ bit homomorphically encrypted blinded values, where $\sigma$ is the statistical security parameter (cf. \$2.1.1.2). As $\left\lfloor\frac{T-1}{\sigma+\ell^{\prime}+1}\right\rfloor$ values can be packed into one Paillier ciphertext, $m^{\prime}=\left\lceil M /\left\lfloor\frac{T-1}{\sigma+\ell^{\prime}+1}\right\rfloor\right\rceil$. The resulting communication complexity is $\left(2 M+m^{\prime}\right) \cdot 2 T+2 M \ell^{\prime} \cdot 2(2 t+1)$ bits. The computation complexity of the protocol is $\mathcal{O}\left(\ell^{\prime} M\right)$ public-key operations for each party.

\subsubsection{A More Improved Hybrid Minimum Protocol}

We improve the hybrid Minimum protocol of $\$ 5.3 .3 .3$ by exploiting the observation of $\$ 5.3 .3 .4$ that the distances can be compared first with the threshold and the minimum is determined afterwards. This results in a smaller circuit and hence better efficiency of the protocol.

The resulting Minimum protocol works as follows: After converting the vector of HEs $\llbracket D_{1} \rrbracket, . ., \llbracket D_{M} \rrbracket$ into ints garbled equivalent $\widetilde{D}_{1}, . ., \widetilde{D}_{M}$, each garbled value is compared with the threshold value $\tau$ using $M$ comparison circuits (cf. \$3.3.3.1). The result is a vector of $M$ garbled bits whose components are encryptions of 1 for each matched entry. Afterwards, the leftmost maximum value and index are determined using a corresponding circuit (cf. 3.3 .3 .3 ) and output to $\mathcal{C}$. If the maximum value is 0 , then no match was found. Otherwise, the maximum index is the id of the matched entry.

The TASTYL code for the privacy-preserving face recognition protocol using the improved Minimum protocol is listed in Fig. 5.9.

\footnotetext{
${ }^{10}$ Although the outcomes of the comparison do not reveal the identities of the matching entries as they are permuted randomly (see final note in observation $\left[\mathrm{BBC}^{+} 10\right.$, Sect. 5.3]), they do reveal the cardinality of matching entries.
} 


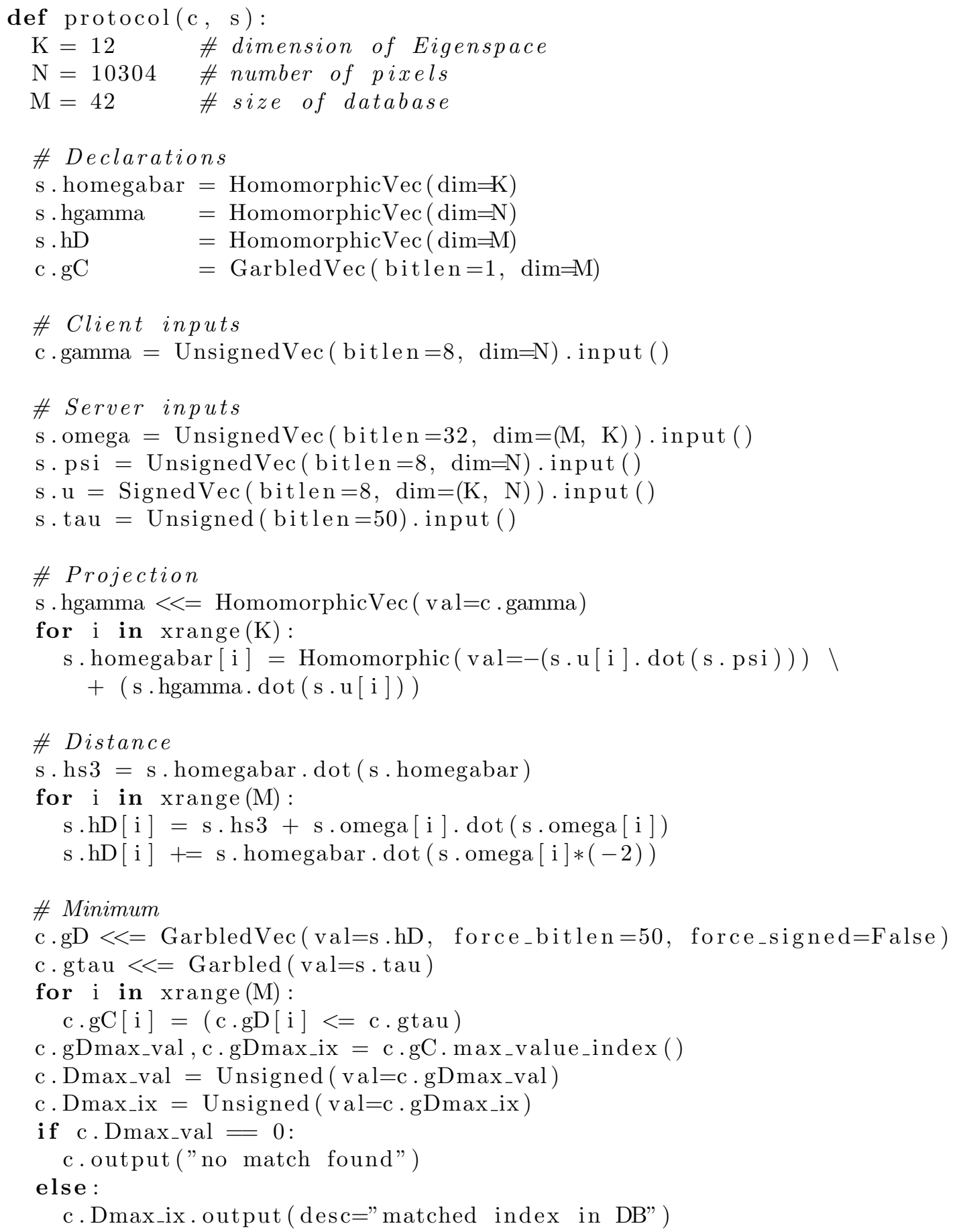

Figure 5.9: TASTYL: More Improved Privacy-Preserving Face Recognition 


\subsubsection{Protocol Complexity}

We determine the complexity of our more improved Minimum protocol described above: In addition to the $M$ comparison circuits of size $\ell^{\prime}$ non-XOR gates each (cf. \$3.3.3.1) the circuit for computing the maximum value and index of 1-bit values requires $2 M$ non-XOR gates (cf. 33.3 .3 .3$)$. This results in $M\left(\ell^{\prime}+2\right)$ non-XOR gates.

The $M$ homomorphically encrypted $\ell^{\prime}$-bit values can be converted into their garbled equivalents with the protocol of $\$ 5.1 .3 .2$. This protocol requires to evaluate a circuit with $M \ell^{\prime}$ non-XOR gates, send $M \ell^{\prime}$ garbled bits from $\mathcal{S}$ to $\mathcal{C}$, and execute an $\ell^{\prime} M$ parallel Oblivious Transfer (OT) protocol (cf. \$2.2.3). Additionally, this protocol requires to send $m$ packed Paillier ciphertexts, where $m=\left\lceil M /\left\lfloor\frac{T-1-\sigma}{\ell^{\prime}}\right\rfloor\right\rceil$ as each packed ciphertext can contain up to $\left\lfloor\frac{T-1-\sigma}{\ell^{\prime}}\right\rfloor$ values. Using the OT protocol of $\$ 2.2 .3 .4$ and garbled row reduction [NPS99] with free XORs [KS08a] for GCs (cf. 32.2.2.3) we obtain the following communication complexity in the Random Oracle (RO) model:

With pre-computations. When pre-computations are allowed, most of the communication complexity can be shifted into the setup phase: $\mathrm{OTs}\left(\approx 4 M \ell^{\prime} t+6 t^{2} \mathrm{bits}\right)$, transfer of the $\mathrm{GC}\left(3(t+1) \cdot 2 M\left(\ell^{\prime}+1\right)\right.$ bits $)$, and transfer of garbled values $((t+$ 1) $M \ell^{\prime}$ bits). The resulting communication complexity of the setup phase is $\approx 6 t^{2}+$ $11 M \ell^{\prime} t$ bits. In the highly efficient online phase, only $m$ Paillier ciphertexts are sent ( $2 m T$ bits), and the online phase of the OT protocol is executed (2M $\ell^{\prime} t$ bits).

Without pre-computations. In ad-hoc application scenarios, where $\mathcal{C}$ and $\mathcal{S}$ meet spontaneously, no pre-computations are possible. In this case the communication complexity of our Minimum protocol consists of the above setup- and online communication complexity without the online OT protocol.

\subsubsection{Performance Comparison}

Finally, we compare the Minimum protocols presented above with respect to their communication and computation complexity.

Communication Complexity. We choose the size of security parameters according to $\$ 2.1 .1 .2$ and set the statistical security parameter to $\sigma=80$. For the bit length $\ell^{\prime}$ of the values $D_{i}$ we consider two application scenarios: Eigenface-based face recognition of $\left[\mathrm{EFG}^{+} 09\right]\left(\ell^{\prime}=50\right)$ as shown in Fig. 5.10 on page 126 and Fingerprint matching of $\left[\mathrm{BBC}^{+} 10\right]\left(\ell^{\prime}=19\right)$ as shown in Fig. 5.11 on page 127 . In particular we compare the values for a database with $M=4500$ entries.

We observe that the Minimum protocol of [EFG+09] (\$5.3.3.2) requires substantially more communication than the other protocols (by factor of 4 to 5 ).

For our more improved hybrid protocol of \$5.3.4 we see that, if pre-computations are possible, the online phase is substantially more efficient than the setup phase and 
the protocol of $\left[\mathrm{BBC}^{+} 10\right.$, Sect. 4.4.1] $(\$ 5.3 .3 .4)$ which does not allow to move any communication into a setup phase (by factor of 5 ). In the Ad-Hoc scenario, where no pre-computations are possible, the communication complexity of our protocol is only slightly larger than that of the setup phase in case of pre-computations.

The relation between the communication complexity of our Ad-Hoc protocol and that of the protocol of $\left[\mathrm{BBC}^{+} 10\right.$, Sect. 4.4.1] depends on the bit length $\ell^{\prime}$ : in the face-recognition scenario $\left(\ell^{\prime}=50\right)$ our protocol is slightly less efficient (cf. Fig. 5.10), whereas in the fingerprint matching scenario $\left(\ell^{\prime}=19\right)$ our improved Minimum protocol of $\$ 5.3 .4$ is even more efficient than that of $\left[\mathrm{BBC}^{+} 10\right.$, Sect. 4.4.1]. For increasing security level we observe that the communication complexity of the HE-based protocols is affected more than that of our hybrid protocol as the asymmetric security parameter $T$ grows faster than the symmetric one $t$ (cf. $\$ 2.1 .1 .2$ ).

Computation Complexity. In all Minimum protocols presented before, the $M$ Paillier ciphertexts need to be processed, where the workload in our hybrid protocols is smaller than that of the HE-based ones due to packing. Besides the Paillier operations the following computations are needed.

In both $\mathrm{HE}$-based Minimum protocols $\left[\mathrm{EFG}^{+} 09, \mathrm{BBC}^{+} 10\right]$, both $\mathcal{C}$ and $\mathcal{S}$ need to perform $\mathcal{O}\left(\ell^{\prime} M\right)$ public key operations in the underlying homomorphic cryptosystem (DGK respectively EC-ElGamal) during the online phase.

When pre-computations are possible, the online phase of our hybrid Minimum protocols requires only $\mathcal{C}$ to perform $\mathcal{O}\left(\ell^{\prime} M\right)$ symmetric cryptographic operations $($ SHA-256 evaluations), while $\mathcal{S}$ performs only XORs of bitstrings for online OT (cf. \$2.2.3.3). In the setup phase (or additionally in the online phase when pre-computations are not possible), $\mathcal{S}$ performs $\mathcal{O}\left(\ell^{\prime} M\right)$ symmetric cryptographic operations (SHA-256 evaluations) to generate the GC. For OT, both parties perform only $\mathcal{O}(t)$ public-key operations (EC multiplications) and $\mathcal{O}\left(\ell^{\prime} M\right)$ symmetric cryptographic operations (SHA-256 evaluations) as described in $\$ 2.2 .3 .4$. As SHA 256 can be evaluated more efficiently than public-key operations, the computation complexity of our hybrid protocols is lower than that of the HE-based protocols for sufficiently large databases $\left(\ell^{\prime} M>t\right)$.

Our implementation results in [SSW10] show that, with pre-computations, the online phase of the hybrid face-recognition protocol of $\$ 5.3 .3 .3$ (and hence also that of the more improved protocol of $\$ 5.3 .4$ is substantially faster than that of the HE-only protocols of $\left[\mathrm{EFG}^{+} 09, \mathrm{BBC}^{+} 10\right]$ and scales well with increasing security level, even when implemented in the relatively slow Python programming language (compared to optimized $\mathrm{C}++$ in which the HE-based protocols were implemented). For $\ell^{\prime} M=320$, runtimes on an Intel Core 2 Duo at $2.4 \mathrm{GHz}$ were $18 \mathrm{~s}$ [EFG $\mathrm{Eg}^{+}$], $16 \mathrm{~s}$ [BBC $\left.{ }^{+} 10\right]$, and $8 \mathrm{~s}$ [SSW10], respectively. 


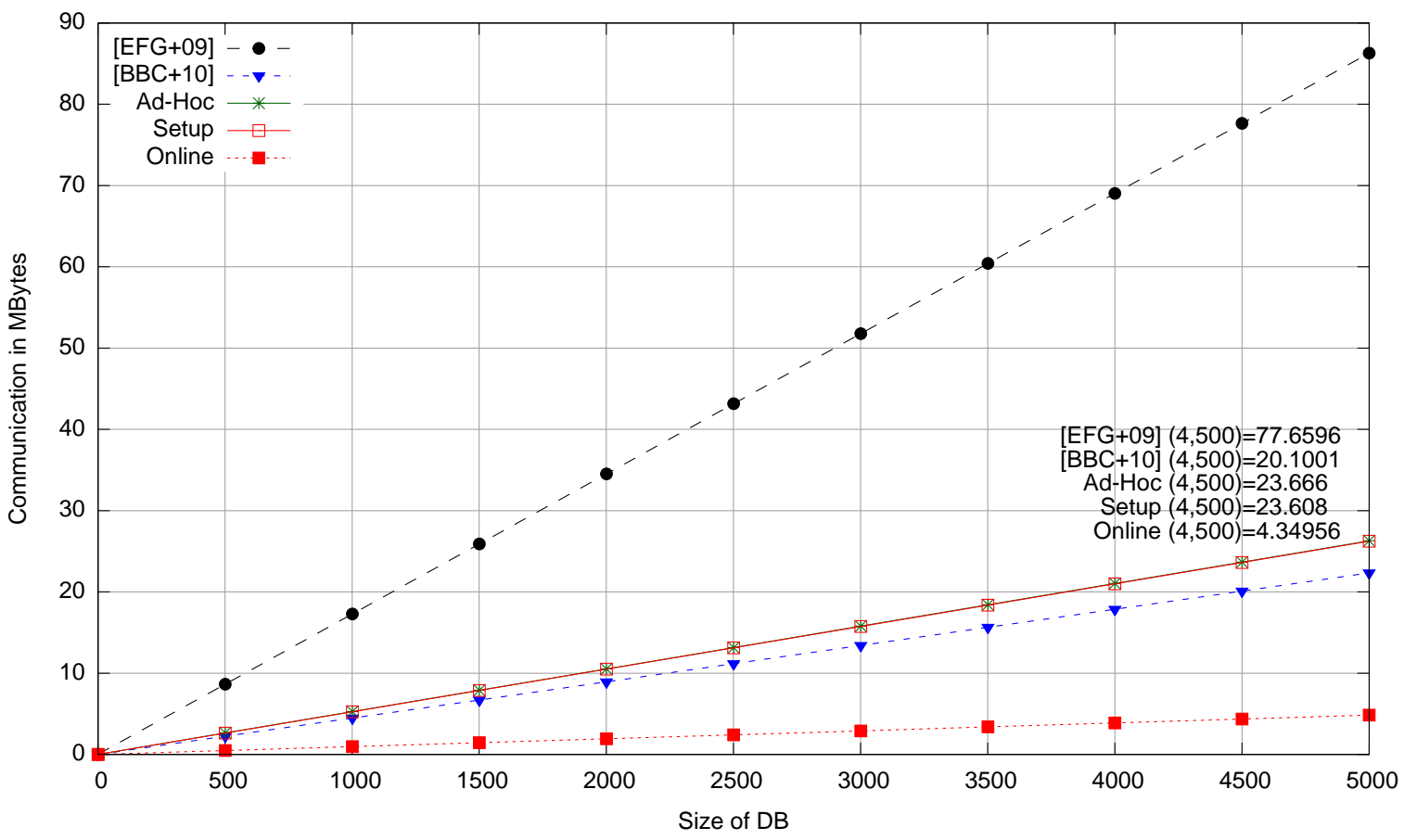

(a) ultra-short-term security

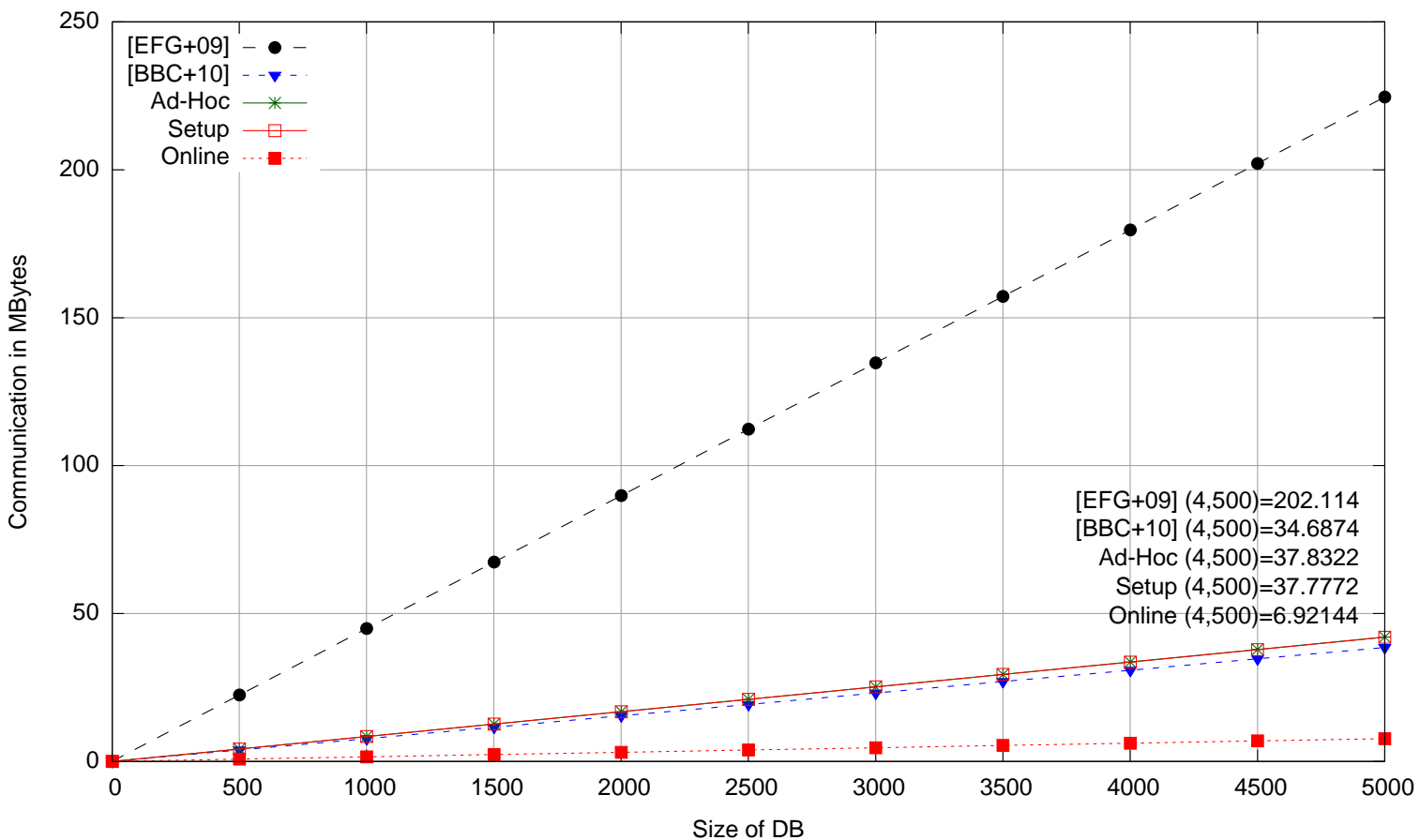

(b) long-term security

Figure 5.10: Minimum: Communication Complexity (Face-Recognition) 


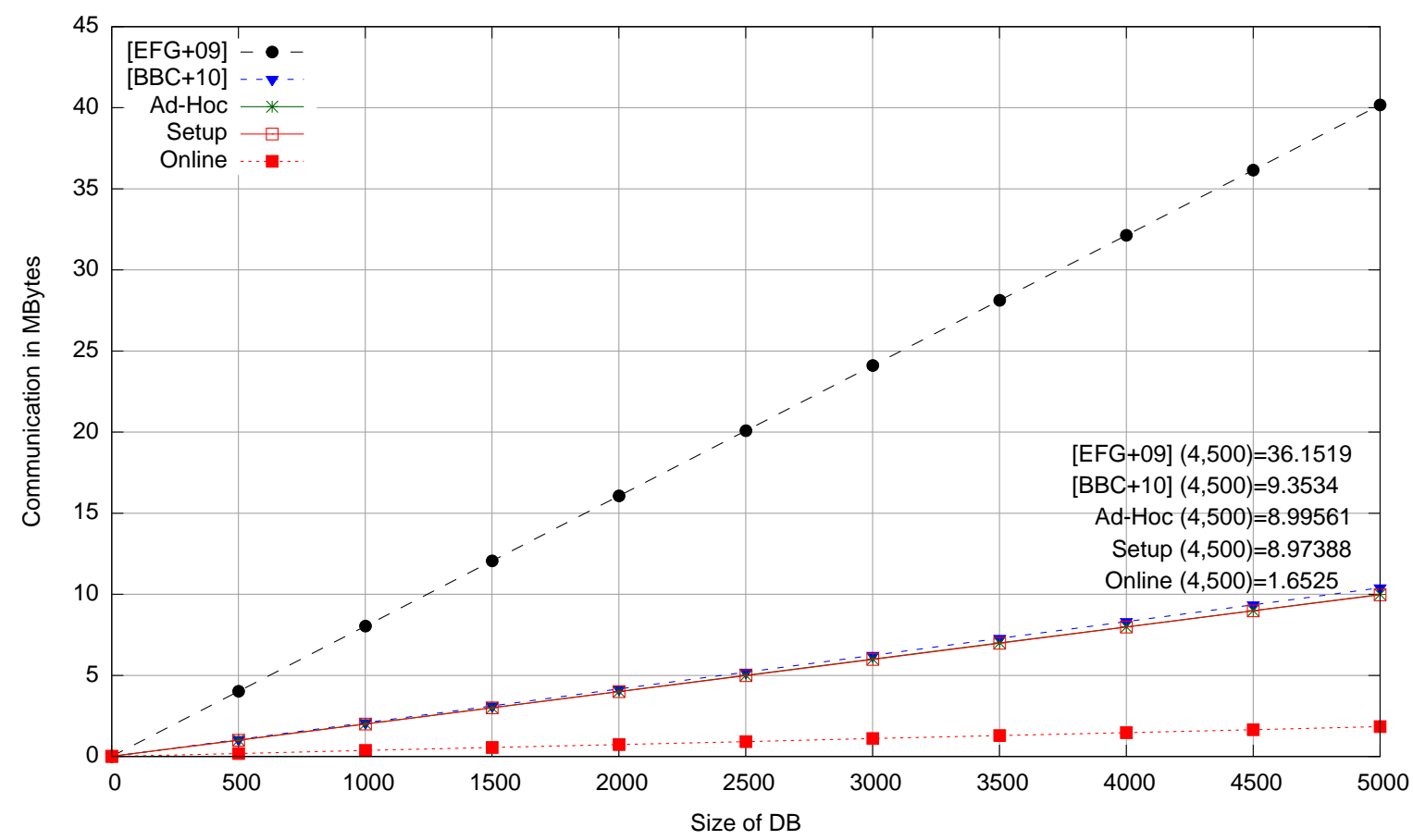

(a) ultra-short-term security

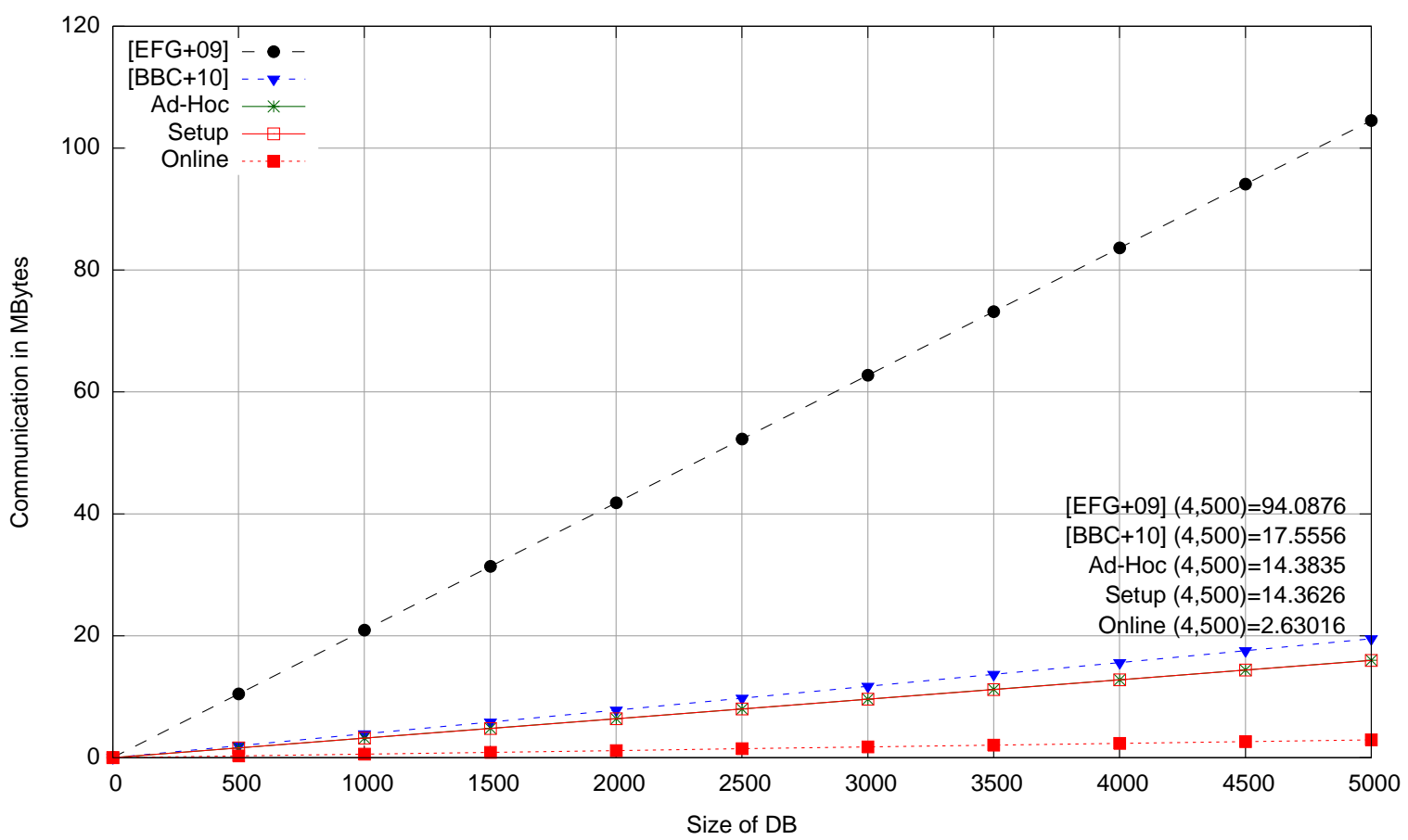

(b) long-term security

Figure 5.11: Minimum: Communication Complexity (Fingerprint Matching) 


\section{Chapter 6}

\section{Conclusion}

In this chapter we summarize the findings of this thesis in $\$ 6.1$ (see also $\$ 1.1$ for the outline and $\$ 1.2$ for the main results) before concluding with possible directions for future research in $\S 6.2$.

\subsection{Summary}

SFE using HE vs. GC, Two-party Secure Function Evaluation (SFE) protocols can be constructed based on either Homomorphic Encryption (HE) or Garbled Circuits $(\mathrm{GCs})$ which we have summarized in detail in \$2. Each of these techniques has its respective advantages and disadvantages: Concerning communication, $\mathrm{HE}$ allows to operate directly on the ciphertexts, whereas for GC-based SFE, helper information in form of the GC needs to be transferred for each operation (this can be done already in a setup phase). With regard to computation, $\mathrm{HE}$ requires computationally expensive public-key operations (these can be partly pre-computed) whereas GC is mostly based on faster symmetric-key operations - indeed, GC-based SFE protocols enable to shift most of the complexity into a less time-critical setup phase and require only a constant (in the security parameter) number of public-key operations $(2.3 .1 .1)$.

Use HE or GC? Today's most efficient GC constructions allow "free XORs", i.e., secure evaluation of XOR gates requires no transfer of garbled tables and only negligible computation (\$2.2.2.3). The performance of GC-based SFE protocols can be improved by exploiting free XORs, e.g., replacing costly non-XOR gates with smaller gates and some free XORs $(\$ 3.2)$. These optimizations result in improved circuit constructions for many standard functionalities (\$3.3). Using these optimized circuits we have shown that GCs allow for more efficient secure comparison and first-price auctions (\$3.4), whereas $\mathrm{HE}$ is suited better for secure ( $\ell$-bit integer) multiplication $(\$ 5.2 .5 .1)$ as publickey operations are faster than transferring and evaluating a large GC for multiplication with $\mathcal{O}\left(\ell^{1.6}\right)$ gates $(3.3 .2 .2$.

Use HW with GC. If available, hardware (HW) can be used to enhance GC-based protocols in several ways. A tamper-proof $\mathrm{HW}$ token issued by the server can be used 
to locally generate GCs for the client which completely eliminates the need to transfer GCs over the Internet (\$4.1). A similar token can be used for secure outsourcing of computations and arbitrary computations in a cloud computing scenario where fast response times are needed $(4.3)$. GCs can also be evaluated efficiently in $\mathrm{HW}$ which enables leakage-resilient One-Time Programs (OTPs) (\$4.2).

Use $\mathrm{HE}$ and GC! The combination of both SFE techniques, $\mathrm{HE}$ and GC, in our modular SFE framework allows to construct highly efficient SFE protocols that combine the advantages of both techniques (\$5.1). Our novel SFE compiler, called Tool for Automating Secure Two-partY computations (TASTY) (\$5.2), implements this framework and makes it accessible to non-experts. We consider privacy-preserving face recognition based on Eigenfaces as representative example application that benefits from the combination of $\mathrm{HE}$ and $\mathrm{GC}(\$ 5.3$.

\subsection{Directions for Future Research}

Finally, we give some directions for future research in engineering Secure Function Evaluation (SFE) protocols for practical applications that can build upon and continue beyond the fundaments laid in this thesis.

In the future we hope that SFE will be increasingly used in real-life applications where privacy needs to be preserved, particularly in the e-health sector. Before SFE can be used in a specific application, it needs to be investigated, whether the efficiency of SFE is sufficient. On the other hand, before the functionalities to be evaluated in the specific target applications are known, it is impossible to identify the bottlenecks of current most efficient SFE constructions that need to be optimized further. This chickenand-egg problem can be tackled with techniques from algorithm engineering [San09] in a step-wise refinement process: starting from some target applications a generic tool is built with which new applications are evaluated. The encountered restrictions and bottlenecks of the tool show what aspects need to be optimized in a next version of the tool and so on. With the Tool for Automating Secure Two-partY computations (TASTY) presented in $\$ 5.2$ we went through the first cycle of this process: We started with a few applications including privacy-preserving face recognition (\$5.3). Meanwhile, we have preliminary results including the use of TASTY for a new application privacy-preserving classification of medical ECG data $\left[\mathrm{BFK}^{+} 09 \mathrm{~b}, \mathrm{BFK}^{+} 09 \mathrm{a}, \mathrm{SS} 10 \mathrm{a}\right]$.

For future applications we expect that the functionalities that need to be evaluated securely will be substantially larger and more complex. Additionally, system requirements, e.g., for mobile devices, will be more stringent than in the applications considered so far. Therefore, we focus on SFE of large functionalities (\$6.2.1) and automatic partitioning of complex functionalities $(86.2 .2)$ in the following. 


\subsubsection{SFE of Large Functionalities}

Today's tools for Garbled Circuit (GC)-based SFE (e.g., MNPS04, LPS08, PSSW09, $\left.\mathrm{HKS}^{+} 10\right]$ ) store the entire functionality and intermediate values during its evaluation in primary memory which currently limits the size of circuits that can be evaluated to a few million gates (approximately 4 Mio. in Fairplay and 8 Mio. in TASTY, see full version of $\left.\left[\mathrm{HKS}^{+} 10\right]\right)$. Without fundamental changes of the architecture, evaluation of larger functionalities could be achieved by using virtual memory, i.e., transparently swapping into slower but substantially larger secondary memory, which, however, would have severe impact on the performance. The possibility and associated problems of using secondary memory applies to SFE implementations in software (SW) as well as in hardware (HW): SW implementations, run on today's PCs, have access to Gigabytes of primary memory (RAM) and Terabytes of secondary memory (hard disk storage). Today's smartphones have only hundreds of Megabytes of primary memory (RAM) and a few Gigabytes of secondary (FLASH) memory. For HW implementations in embedded systems, memory access is even more strict as primary memory (registers and on-chip memory) is rare while access to secondary (off-chip) memory is relatively expensive. Indeed, memory access was the major bottleneck of our FPGAbased implementation for GC evaluation as discussed in $\$ 4.2 .3 .4$.

Streaming. To overcome these memory restrictions, a streamlined evaluation is needed that never holds the entire functionality in memory as follows: Firstly, the circuit can be compiled on-the-fly using a constant amount of memory as implemented in TASTY (see discussion in full version of $\left[\mathrm{HKS}^{+} 10\right]$ ). Further, this stream of gates can be directly combined with our constant-memory $\mathrm{GC}$ creation technique of \$4.1.4.3. and the garbled tables can be streamed directly over the network 1 to the evaluator who evaluates them on-the-fly as suggested in 4.1 .4 .1 and $\$ 4.2$. The intermediate values during evaluation can be cached as described and used in 84.2 .3 . Finally, Oblivious Transfer (OT) can be extended dynamically as mentioned in [IKNP03. s.t. only a constant (in the security parameter) number of public key operations is needed for an arbitrary (and unknown in advance) number of OTs. We note, however, that some circuits cannot be streamed as their evaluation requires memory linear in the circuit size as described in 4.2.3.1.

For Secure Multi-Party Computation (SMPC) based on Homomorphic Encryption (HE), streaming was implemented already in the VIFF framework [DGKN09]. The recently proposed VMCrypt library [MK10] specifically aims to maximize GC streaming in the two-party setting. The techniques described above as well as our framework for modular SFE that combines the advantages of $\mathrm{HE}$ and $\mathrm{GC}(\$ 5.1)$ can be used with their architecture, resulting in corresponding performance improvements.

\footnotetext{
${ }^{1}$ Alternatively, when pre-computations should be used, the GC can be buffered sequentially in secondary memory.
} 
Parallelism. In addition to streaming, the computations in SFE protocols could also be performed in parallel to exploit the large variety of capabilities for multi-processing available today. Parallelization can be done either on one system (e.g., using multicore CPUs, general purpose Graphics Processing Units (GPUs), stream processors such as the Cell Broadband Engine Architecture, or Field-Programmable Gate Arrays (FPGAs) , or even distributed among multiple systems in a grid or outsource it into the cloud ${ }^{2}$ The allocation of computing resources could even be performed dynamically during runtime depending on the system load (e.g., dynamic allocation of multi-core CPUs and GPUs in a grid [Bla10]).

To allow this parallelization, it needs to be investigated how to partition the evaluated function and how to allocate sub-computations to the different computing devices efficiently.

\subsubsection{Automatic Partitioning into Hybrid SFE Protocols}

As soon as the evaluated functions grow larger and more complex, it will be infeasible to manually find a good partition into sub-functionalities that can be evaluated securely in our modular SFE framework of \$5.1. Instead, the functionality to be evaluated should be input in a function description language (cf. \$5.2.1.3) and tools should automatically determine a good partitioning into SFE sub-protocols, targeted for the specific system on which the protocol should be deployed. Possible optimization parameters could be the total amount of communication, availability of $\mathrm{HW}$ accelerators for $\mathrm{GC}$ evaluation $(\$ 4.2 .3$, trusted $\mathrm{HW}$ for GC creation (\$4.1), parallel computing devices and memory (\$6.2.1), network bandwidth, or even power consumption for mobile devices. The tool could automatically choose the most efficient SFE sub-protocols, what to compute where, and whether to use pre-computations or streaming. The partitioning could even be done adaptively depending on the current system state such as load or battery level. As a first step into this direction, a compiler could be built on top of TASTY ( $\$ 5.2$ ) that automatically compiles a high-level function description into different TASTY input Language (TASTYL) programs. Afterwards, the most efficient protocol could be selected among the different choices using TASTY's benchmarking capabilities.

\footnotetext{
${ }^{2}$ Today's high performance computing services offered by cloud providers even include high-end
} GPUs, e.g., AMEb. 


\section{Bibliography}

[AARR02] Dakshi Agrawal, Bruce Archambeault, Josyula R. Rao, and Pankaj Rohatgi. The EM side-channel(s). In Cryptographic Hardware and Embedded Systems (CHES'O2), volume 2523 of $L N C S$, pages 29-45. Springer, 2002.

[AB06] Shai Avidan and Moshe Butman. Efficient methods for privacy preserving face detection. In Advances in Neural Information Processing Systems (NIPS'06), pages 57-64. MIT Press, 2006.

$\left[\mathrm{ABB}^{+} 10\right] \quad$ José Bacelar Almeida, Endre Bangerter, Manuel Barbosa, Stephan Krenn, Ahmad-Reza Sadeghi, and Thomas Schneider. A certifying compiler for zero-knowledge proofs of knowledge based on sigma-protocols. In 15th European Symposium on Research in Computer Security (ESORICS'10), volume 6345 of LNCS, pages 151-167. Springer, September 20-22, 2010. Full version available at http://eprint.iacr.org/2010/339.

[ACCK01] Joy Algesheimer, Christian Cachin, Jan Camenisch, and Günter Karjoth. Cryptographic security for mobile code. In IEEE Symposium on Security and Privacy (SEP'01), pages $2-11,2001$.

[AG01] Mehdi-Laurent Akkar and Christophe Giraud. An implementation of DES and AES, secure against some attacks. In Cryptographic Hardware and Embedded Systems (CHES'01), volume 2162 of LNCS, pages 309-318. Springer, 2001.

[AHI11] Benny Applebaum, Danny Harnik, and Yuval Ishai. Semantic security under related-key attacks and applications. In Innovations in Computer Science (ICS'11), 2011. To appear. Preliminary version available at http://eprint.iacr.org/2010/544,

[AIR01] William Aiello, Yuval Ishai, and Omer Reingold. Priced oblivious transfer: How to sell digital goods. In Advances in Cryptology - EUROCRYPT'01, volume 2045 of LNCS, pages 119-135. Springer, 2001.

[AL07] Yonatan Aumann and Yehuda Lindell. Security against covert adversaries: Efficient protocols for realistic adversaries. In Theory of Cryptography (TCC'07), volume 4392 of LNCS, pages 137-156. Springer, 2007.

[ALR99] Eric Allender, Michael C. Loui, and Kenneth W. Regan. Complexity classes. In Mikhail J. Atallah, editor, Algorithms and Theory of Computation Handbook, chapter 27. CRC Press, 1999.

[AmEa] Amazon Elastic Compute Cloud (EC2). http://aws.amazon.com/ec2.

[AMEb] Amazon High Performance Computing (HPC). http://aws.amazon.com/ec2/ hpc-applications/.

[AMP04] Gagan Aggarwal, Nina Mishra, and Benny Pinkas. Secure computation of the $\mathrm{k}$ thranked element. In Advances in Cryptology - EUROCRYPT'04, volume 3027 of LNCS, pages 40-55. Springer, 2004.

[AmS] Amazon Simple Storage Service (S3). http://aws.amazon.com/s3 
[APRS01] Mikhail J. Atallah, K. N. Pantazopoulos, John R. Rice, and Eugene H. Spafford. Secure outsourcing of scientific computations. Advances in Computers, 54:216-272, 2001.

$\left[\mathrm{BBB}^{+} 09\right]$ Endre Bangerter, Manuel Barbosa, Daniel J. Bernstein, Ivan Damgård, Dan Page, Jakob I. Pagter, Ahmad-Reza Sadeghi, and Sampo Sovio. Using compilers to enhance cryptographic product development. In Information Security Solutions Europe (ISSE'09), pages 291-301. Vieweg+Teubner, January 2009.

$\left[\mathrm{BBC}^{+} 10\right]$ Mauro Barni, Tiziano Bianchi, Dario Catalano, Mario Di Raimondo, Ruggero Donida Labati, Pierluigi Failla, Dario Fiore, Riccardo Lazzeretti, Vincenzo Piuri, Fabio Scotti, and Alessandro Piva. Privacy-preserving fingercode authentication. In ACM Workshop on Multimedia and Security (MMESec'10), pages 231-240. ACM, 2010. Corrected version available at http://www.dmi.unict.it/diraimondo/uploads/papers/ fingercode-protocol.pdf.

$\left[\mathrm{BBH}^{+} 09\right]$ Endre Bangerter, Thomas Briner, Wilko Henecka, Stephan Krenn, Ahmad-Reza Sadeghi, and Thomas Schneider. Automatic generation of sigma-protocols. In 6th European Workshop on Public Key Services, Applications and Infrastructures (EUROPKI'09), volume 6391 of $L N C S$, pages 67-82. Springer, September 10-11, 2009. Implementation available at http://www. cace-project.eu/index.php?Itemid=15.

$\left[\mathrm{BBK}^{+} 09\right]$ Endre Bangerter, Stefania Barzan, Stephan Krenn, Ahmad-Reza Sadeghi, Thomas Schneider, and Joe-Kai Tsay. Bringing zero-knowledge proofs of knowledge to practice. In 17th International Workshop on Security Protocols (SPW'09), April 1-3, 2009. Full version available at http://eprint.iacr.org/2009/211.

[BBP91] Joan Boyar, Gilles Brassard, and René Peralta. Subquadratic zero-knowledge. In Foundations of Computer Science (FOCS'91), pages 69-78. IEEE, 1991.

[BBP95] Joan Boyar, Gilles Brassard, and René Peralta. Subquadratic zero-knowledge. Journal of the ACM, 42(6):1169-1193, 1995.

[BC86] Gilles Brassard and Claude Crépeau. Zero-knowledge simulation of boolean circuits. In Advances in Cryptology - CRYPTO'86, volume 263 of LNCS, pages 223-233. Springer, 1986.

$\left[\mathrm{BCG}^{+}\right.$06] Stefan Berger, Ramón Cáceres, Kenneth A. Goldman, Ronald Perez, Reiner Sailer, and Leendert v. Doorn. vTPM: Virtualizing the Trusted Platform Module. In USENIX Security Symposium (Security'06), pages 305-320. USENIX, 2006.

$\left[\mathrm{BCK}^{+} 09\right]$ Endre Bangerter, Jan Camenisch, Stephan Krenn, Ahmad-Reza Sadeghi, and Thomas Schneider. Automatic generation of sound zero-knowledge protocols. 28th Advances in Cryptology - EUROCRYPT 2009 Poster Session, April 26-30, 2009. Full version available at http://eprint.iacr.org/2008/471

$\left[\mathrm{BDK}^{+} 05\right]$ Xavier Boyen, Yevgeniy Dodis, Jonathan Katz, Rafail Ostrovsky, and Adam Smith. Secure remote authentication using biometric data. In Advances in Cryptology - EUROCRYPT'05, volume 3494 of LNCS, pages 147-163. Springer, 2005.

[BDNP08] Assaf Ben-David, Noam Nisan, and Benny Pinkas. FairplayMP: a system for secure multiparty computation. In ACM Conference on Computer and Communications Security (CCS'08), pages 257-266. ACM, 2008. http://fairplayproject.net/fairplayMP. html.

[BDP00] Joan Boyar, Ivan Damgård, and René Peralta. Short non-interactive cryptographic proofs. Journal of Cryptology, 13(4):449-472, 122000. 
[Bea95] Donald Beaver. Precomputing oblivious transfer. In Advances in Cryptology CRYPTO'95, volume 963 of LNCS, pages 97-109. Springer, 1995.

[Ber91] C. Leonard Berman. Circuit width, register allocation, and ordered binary decision diagrams. IEEE Trans. on CAD of Integrated Circuits and Systems, 10(8):1059-1066, 1991.

[BFK ${ }^{+}$09a] Mauro Barni, Pierluigi Failla, Vladimir Kolesnikov, Riccardo Lazzeretti, Annika Paus, Ahmad-Reza Sadeghi, and Thomas Schneider. Efficient privacy-preserving classification of ECG signals. In 1st IEEE International Workshop on Information Forensics and Security (IEEE WIFS'09), pages 91-95. IEEE, December 6-9, 2009.

$\left[\mathrm{BFK}^{+}\right.$09b] Mauro Barni, Pierluigi Failla, Vladimir Kolesnikov, Riccardo Lazzeretti, Ahmad-Reza Sadeghi, and Thomas Schneider. Secure evaluation of private linear branching programs with medical applications. In 14th European Symposium on Research in Computer Security (ESORICS'09), volume 5789 of LNCS, pages 424-439. Springer, September 21-25, 2009. Full version available at http://eprint.iacr.org/2009/195.

$\left[\mathrm{BFL}^{+} 11\right] \quad$ Mauro Barni, Pierluigi Failla, Riccardo Lazzeretti, Ahmad-Reza Sadeghi, and Thomas Schneider. Privacy-preserving ECG classification with branching programs and neural networks. IEEE Transactions on Information Forensics and Security (TIFS), 2011. Accepted for publication.

$\left[\mathrm{BGJ}^{+} 05\right]$ Anthony Bussani, John L. Griffin, Bernhard Jasen, Klaus Julisch, Guenter Karjoth, Hiroshi Maruyama, Megumi Nakamura, Ronald Perez, Matthias Schunter, Axel Tanner, Leendert v. Doorn, Els A. v. Herreweghen, Michael Waidner, and Sachiko Yoshihama. Trusted Virtual Domains: Secure Foundations for Business and IT Services. Technical Report Research Report RC23792, IBM Research, November 2005.

[BGN05] Dan Boneh, Eu-Jin Goh, and Kobbi Nissim. Evaluating 2-DNF formulas on ciphertexts. In Theory of Cryptography Conference (TCC'05), volume 3378 of LNCS, pages 325-341. Springer, 2005.

[BGW88] Michael Ben-Or, Shafi Goldwasser, and Avi Wigderson. Completeness theorems for non-cryptographic fault-tolerant distributed computation. In Symposium on Theory of Computing (STOC'88), pages 1-10. ACM, 1988.

[BK04] Ian F. Blake and Vladimir Kolesnikov. Strong conditional oblivious transfer and computing on intervals. In Advances in Cryptology - ASIACRYPT'04, volume 3329 of LNCS, pages 515-529. Springer, 2004.

[BKSS10] Endre Bangerter, Stephan Krenn, Ahmad-Reza Sadeghi, and Thomas Schneider. YAZKC: Yet Another Zero-Knowledge Compiler. 19th USENIX Security Symposium (Security'10) Poster Session, August 11-13, 2010.

[Bla10] Thorsten Blass. Multi-GPU Cluster use for Java/OpenMP. Master's thesis, FriedrichAlexander University Erlangen-Nürnberg, Germany, 2010.

[BLP93] Joan Boyar, Carsten Lund, and René Peralta. On the communication complexity of zero-knowledge proofs. J. Cryptology, 6(2):65-85, 1993.

[BLW08] Dan Bogdanov, Sven Laur, and Jan Willemson. Sharemind: A framework for fast privacypreserving computations. In European Symposium on Research in Computer Security (ESORICS'08), volume 5283 of LNCS, pages 192-206. Springer, 2008.

[Bow] Owen Bowcott. Interpol wants facial recognition database to catch suspects. Guardian (October 20, 2008), http://www.guardian.co.uk/world/2008/oct/20/ interpol-facial-recognition. 
[BP96] Joan Boyar and René Peralta. Short discrete proofs. In Advances in Cryptology EUROCRYPT'96, volume 1070 of LNCS, pages 131-142. Springer, 1996.

[BP06] Joan Boyar and René Peralta. Concrete multiplicative complexity of symmetric functions. In Mathematical Foundations of Computer Science (MFCS'06), volume 4162 of LNCS, pages 179-189. Springer, 2006.

[BP10] Joan Boyar and René Peralta. A new combinational logic minimization technique with applications to cryptology. In Symposium on Experimental Algorithms (SOA'10), volume 6049 of $L N C S$, pages 178-189. Springer, 2010.

[BPP00] Joan Boyar, René Peralta, and Denis Pochuev. On the multiplicative complexity of Boolean functions over the basis $(\wedge, \oplus, 1)$. Theoretical Computer Science, 235(1):43-57, 2000 .

[BPSW07] Justin Brickell, Donald E. Porter, Vitaly Shmatikov, and Emmett Witchel. Privacypreserving remote diagnostics. In ACM Computer and Communications Security (CCS'07), pages 498-507. ACM, 2007.

[BR93] Mihir Bellare and Phillip Rogaway. Random oracles are practical: A paradigm for designing efficient protocols. In ACM Conference on Computer and Communications Security (CCS'93), pages 62-73. ACM, 1993.

[Bri04] Thomas Briner. Compiler for zero-knowledge proof-of-knowledge protocols. Master's thesis, ETH Zurich, Switzerland, 2004.

[Bry91] Randal E. Bryant. On the complexity of VLSI implementations and graph representations of boolean functions with application to integer multiplication. IEEE Transactions on Computers, 40(2):205-213, 1991.

[BS09] Justin Brickell and Vitaly Shmatikov. Privacy-preserving classifier learning. In Financial Cryptography and Data Security (FC'09), volume 5628 of LNCS, pages 128-147. Springer, 2009.

[BW96] Beate Bollig and Ingo Wegener. Improving the variable ordering of OBDDs is NPcomplete. IEEE Transactions on Computers, 45(9):993-1002, 1996.

$\left[\mathrm{CAC}^{+} 81\right]$ Gregory J. Chaitin, Marc A. Auslander, Ashok K. Chandra, John Cocke, Martin E. Hopkins, and Peter W. Markstein. Register allocation via coloring. Computer Languages, 6(1):47-57, 1981.

[Can01] Ran Canetti. Universally composable security: A new paradigm for cryptographic protocols. In Foundations of Computer Science (FOCS'01), pages 136-145, 2001.

[CCKM00] Christian Cachin, Jan Camenisch, Joe Kilian, and Joy Müller. One-round secure computation and secure autonomous mobile agents. In International Colloquium on Automata, Languages and Programming (ICALP'00), volume 1853 of LNCS, pages 512-523. Springer, 2000.

$\left[\mathrm{CDE}^{+} 10\right]$ Serdar Cabuk, Chris I. Dalton, Konrad Eriksson, Dirk Kuhlmann, HariGovind V. Ramasamy, Gianluca Ramunno, Ahmad-Reza Sadeghi, Matthias Schunter, and Christian Stüble. Towards automated security policy enforcement in multi-tenant virtual data centers. Journal of Computer Security, 18:89-121, 2010.

[CGH04] Ran Canetti, Oded Goldreich, and Shai Halevi. The random oracle methodology, revisited. Journal of the ACM, 51(4):557-594, 2004. 
$\left[\mathrm{CGJ}^{+}\right.$09] Richard Chow, Philippe Golle, Markus Jakobsson, Elaine Shi, Jessica Staddon, Ryusuke Masuoka, and Jesus Molina. Controlling data in the cloud: outsourcing computation without outsourcing control. In ACM Workshop on Cloud Computing Security (CCSW'09), pages 85-90. ACM, 2009.

[CGS08] Nishanth Chandran, Vipul Goyal, and Amit Sahai. New constructions for UC secure computation using tamper-proof hardware. In Advances in Cryptology - EUROCRYPT'08, volume 4965 of $L N C S$, pages 545-562. Springer, 2008.

[CKV10] Kai-Min Chung, Yael Kalai, and Salil Vadhan. Improved delegation of computation using fully homomorphic encryption. In Advances in Cryptology - CRYPTO'10, volume 6223 of $L N C S$, pages 583-501. Springer, 2010.

[Clo10] Cloud Security Alliance (CSA). Top threats to cloud computing, version 1.0. http: //www.cloudsecurityalliance.org/topthreats/csathreats.v1.0.pdf, March 2010.

[CLOS02] Ran Canetti, Yehuda Lindell, Rafail Ostrovsky, and Amit Sahai. Universally composable two-party and multi-party secure computation. In ACM Symposium on Theory of Computing (STOC'02), pages 494-503, 2002.

[CLRS01] Thomas H. Cormen, Charles E. Leiserson, Ronald L. Rivest, and Clifford Stein. Introduction to Algorithms, Second Edition. The MIT Press, September 2001.

[CRS05] Jan Camenisch, Markus Rohe, and Ahmad-Reza Saodeghi. Sokrates - a compiler framework for zero-knowledge protocols. In Western European Workshop on Research in Cryptology (WEWoRC'05), 2005.

[DFI03] Camil Demetrescu, Irene Finocchi, and Giuseppe F. Italiano. Algorithm engineering, algorithmics column. Bulletin of the EATCS, 79:48-63, 2003.

[DGHV10] Marten van Dijk, Craig Gentry, Shai Halevi, and Vinod Vaikuntanathan. Fully homomorphic encryption over the integers. In Advances in Cryptology - EUROCRYPT'10, volume 6110 of $L N C S$, pages 24-43. Springer, 2010.

[DGK07] Ivan Damgård, Martin Geisler, and Mikkel Krøigaard. Efficient and secure comparison for on-line auctions. In Australasian Conference on Information Security and Privacy (ACISP'07), volume 4586 of LNCS, pages 416-430. Springer, 2007.

[DGK08a] Ivan Damgård, Martin Geisler, and Mikkel Krøigaard. A correction to "efficient and secure comparison for on-line auctions". Cryptology ePrint Archive, Report 2008/321, 2008.

[DGK08b] Ivan Damgård, Martin Geisler, and Mikkel Krøigaard. Homomorphic encryption and secure comparison. Journal of Applied Cryptology, 1(1):22-31, 2008.

[DGKN09] Ivan Damgård, Martin Geisler, Mikkel Krøigård, and Jesper B. Nielsen. Asynchronous multiparty computation: Theory and implementation. In Public Key Cryptography (PKC'O9), volume 5443 of $L N C S$, pages 160-179. Springer, 2009. http://viff.dk.

[DJ01] Ivan Damgård and Mats Jurik. A generalisation, a simplification and some applications of Paillier's probabilistic public-key system. In Public-Key Cryptography (PKC'01), LNCS, pages 119-136. Springer, 2001.

[DJ10] Marten van Dijk and Ari Juels. On the impossibility of cryptography alone for privacypreserving cloud computing. In USENIX Workshop on Hot Topics in Security (HotSec'10). USENIX, 2010. 
[DKM11] Nico Döttling, Daniel Kraschewski, and Jörn Müller-Quade. Unconditional and composable security using a single stateful tamper-proof hardware token. In Theory of Cryptography (TCC'11), LNCS. Springer, 2011. To appear.

[DNW09] Ivan Damgård, Jesper Buus Nielsen, and Daniel Wichs. Universally composable multiparty computation with partially isolated parties. In Theory of Cryptography (TCC'09), volume 5444 of $L N C S$, pages 315-331. Springer, 2009.

[DSV10] Maria Dubovitskaya, Alessandra Scafuro, and Ivan Visconti. Efficient non-interactive oblivious transfer with tamper-proof hardware. Cryptology ePrint Archive, Report 2010/509, 2010. http://eprint.iacr.org/.

[ECR10] ECRYPT II. Yearly report on algorithms and keysizes (2010), March 2010. http: //www.ecrypt.eu.org/documents/D.SPA.13.pdf.

[EFG ${ }^{+}$09] Zekeriya Erkin, Martin Franz, Jorge Guajardo, Stefan Katzenbeisser, Inald Lagendijk, and Tomas Toft. Privacy-preserving face recognition. In Privacy Enhancing Technologies Symposium (PETS'09), volume 5672 of LNCS, pages 235-253. Springer, 2009.

[FAL04] Keith B. Frikken, Mikhail J. Atallah, and Jiangtao Li. Hidden access control policies with hidden credentials. In ACM Workshop on Privacy in the Electronic Society (WPES'04), page 27. ACM, 2004.

[FAL06] Keith B. Frikken, Mikhail J. Atallah, and Jiangtao Li. Attribute-based access control with hidden policies and hidden credentials. IEEE Transactions on Computers, 55(10):1259$1270,2006$.

[FAZ05] Keith B. Frikken, Mikhail J. Atallah, and Chen Zhang. Privacy-preserving credit checking. In ACM conference on Electronic Commerce, pages 147-154. ACM, 2005.

$\left[\mathrm{FFP}^{+}\right.$06] Milan Fort, Felix C. Freiling, Lucia Draque Penso, Zinaida Benenson, and Dogan Kesdogan. Trustedpals: Secure multiparty computation implemented with smart cards. In European Symposium on Research in Computer Security (ESORICS'06), volume 4189 of LNCS, pages 34-48. Springer, 2006.

[Fis01] Marc Fischlin. A cost-effective pay-per-multiplication comparison method for millionaires. In Cryptographer's Track at RSA Conference (CT-RSA'01), volume 2020 of LNCS, pages 457-472. Springer, 2001.

[FLA06] Keith B. Frikken, Jiangtao Li, and Mikhail J. Atallah. Trust negotiation with hidden credentials, hidden policies, and policy cycles. In Network and Distributed System Security Symposium (NDSS'06), pages 157-172. The Internet Society, 2006.

[FNP04] Michael J. Freedman, Kobbi Nissim, and Benny Pinkas. Efficient private matching and set intersection. In Advances in Cryptology - EUROCRYPT'04, volume 3027 of LNCS, pages 1-19. Springer, 2004.

[FPRS04] Joan Feigenbaum, Benny Pinkas, Raphael S. Ryger, and Felipe Saint-Jean. Secure computation of surveys. In EU Workshop on Secure Multiparty Protocols (SMP). ECRYPT, 2004.

[FPS $\left.{ }^{+} 11\right] \quad$ Marc Fischlin, Benny Pinkas, Ahmad-Reza Sadeghi, Thomas Schneider, and Ivan Visconti. Secure set intersection with untrusted hardware tokens. In 11th Cryptographers' Track at the RSA Conference (CT-RSA'11), volume 6558 of $L N C S$, pages 1-16. Springer, February 14-18, 2011. To appear. 
[Fri09] Keith B. Frikken. Practical private DNA string searching and matching through efficient oblivious automata evaluation. In Data and Applications Security (DBSec'09), volume 5645 of LNCS, pages 81-94. Springer, 2009.

[Gam85] Taher El Gamal. A public key cryptosystem and a signature scheme based on discrete logarithms. In Advances in Cryptology - CRYPTO'84, volume 196 of LNCS, pages 10-18. Springer, 1985.

[Gen09a] Craig Gentry. A fully homomorphic encryption scheme. PhD thesis, Stanford University, 2009. http://crypto.stanford.edu/craig.

[Gen09b] Craig Gentry. Fully homomorphic encryption using ideal lattices. In ACM Symposium on Theory of Computing (STOC'09), pages 169-178. ACM, 2009.

[GGP10] Rosario Gennaro, Craig Gentry, and Bryan Parno. Non-interactive verifiable computing: Outsourcing computation to untrusted workers. In Advances in Cryptology CRYPTO'10, volume 6223 of LNCS, pages 465-482. Springer, 2010.

[GH10] Craig Gentry and Shai Halevi. Implementing Gentry's fully-homomorphic encryption scheme. Cryptology ePrint Archive, Report 2010/520, 2010. http://eprint.iacr.org/.

[GHV10] Craig Gentry, Shai Halevi, and Vinod Vaikuntanathan. A simple BGN-type cryptosystem from LWE. In Advances in Cryptology - EUROCRYPT'10, volume 6110 of LNCS, pages 506-522. Springer, 2010. Updated version available at http://eprint.iacr.org/2010/ 182 .

[GIS $\left.{ }^{+} 10\right] \quad$ Vipul Goyal, Yuval Ishai, Amit Sahai, Ramarathnam Venkatesan, and Akshay Wadia. Founding cryptography on tamper-proof hardware tokens. In Theory of Cryptography (TCC'10), volume 5978 of LNCS, pages 308-326. Springer, 2010.

[GKM09] Juan A. Garay, Vladimir Kolesnikov, and Rae McLellan. MAC precomputation with applications to secure memory. In Information Security Conference (ISC'09), volume 5735 of $L N C S$, pages 427-442. Springer, 2009.

[GKR08] Shafi Goldwasser, Yael Tauman Kalai, and Guy N. Rothblum. One-time programs. In Advances in Cryptology - CRYPTO'08, volume 5157 of LNCS, pages 39-56. Springer, 2008.

[GM84] Shafi Goldwasser and Silvio Micali. Probabilistic encryption. Journal of Computer and System Sciences, 28(2):270-299, 1984.

[GMO01] Karine Gandolfi, Christophe Mourtel, and Francis Olivier. Electromagnetic analysis: Concrete results. In Cryptographic Hardware and Embedded Systems (CHES'01), volume 2162 of $L N C S$, pages 251-261. Springer, 2001.

[gmpa] GMP - GNU multi precision arithmetic library. http://gmplib.org.

[gmpb] gmpy - multiprecision arithmetic for Python. http://code.google.com/p/gmpy.

[GMS08] Vipul Goyal, Payman Mohassel, and Adam Smith. Efficient two party and multi party computation against covert adversaries. In Advances in Cryptology - EUROCRYPT'08, volume 4965 of $L N C S$, pages 289-306. Springer, 2008.

[GMY04] Juan A. Garay, Philip MacKenzie, and Ke Yang. Efficient and universally composable committed oblivious transfer and applications. In Theory of Cryptography (TCC'04), volume 2951 of $L N C S$, pages 297-316. Springer, 2004.

[GoA] Google App Engine. https://appengine.google.com 
[Gol04] Oded Goldreich. Foundations of Cryptography, volume 2: Basic Applications. Cambridge University Press, 2004. Draft available at http://www .wisdom.weizmann.ac.il/ oded/ foc-vol2.html.

[GQ10] Damien Giry and Jean-Jacques Quisquater. Cryptographic key length recommendation, 2010. http://keylength.com.

[Gro08] Thomas K. Grose. When surveillance cameras talk, 2008. Time Magazine (February 11, 2008), http://www.time.com/time/world/article/0, 8599, 1711972, 00.html.

[GSV07] Juan A. Garay, Berry Schoenmakers, and José Villegas. Practical and secure solutions for integer comparison. In Public Key Cryptography (PKC'O7), volume 4450 of LNCS, pages 330-342. Springer, 2007.

[GT08] Vandana Gunupudi and Stephen R. Tate. Generalized non-interactive oblivious transfer using count-limited objects with applications to secure mobile agents. In Financial Cryptography and Data Security (FC'08), volume 5143 of LNCS, pages 98-112. Springer, 2008.

[HKS $\left.{ }^{+} 10\right]$ Wilko Henecka, Stefan Kögl, Ahmad-Reza Sadeghi, Thomas Schneider, and Immo Wehrenberg. TASTY: Tool for Automating Secure Two-partY computations. In 17th ACM Conference on Computer and Communications Security (CCS'10), pages 451-462. ACM, October 4-8, 2010. Full version available at http://eprint.iacr.org/2010/365. http://tastyproject.net.

[HL08] Carmit Hazay and Yehuda Lindell. Constructions of truly practical secure protocols using standard smartcards. In ACM Conference on Computer and Communications Security (CCS'08), pages 491-500. ACM, 2008.

[HMEK11] Yan Huang, Lior Malka, David Evans, and Jonathan Katz. Efficient privacy-preserving biometric identification. In Network and Distributed System Security (NDSS'11). The Internet Society, 2011. To appear. Code available at http://mightbeevil.org/ secure-biometrics/

[HMU05] Dennis Hofheinz, Jörn Müller-Quade, and Dominique Unruh. Universally composable zero-knowledge arguments and commitments from signature cards. In Central European Conference on Cryptology (MoraviaCrypt'05), 2005.

[HS10] Amir Herzberg and Haya Shulman. Secure guaranteed computation. Cryptology ePrint Archive, Report 2010/449, 2010. http://eprint.iacr.org/

$\left[\mathrm{HSH}^{+} 08\right]$ John A. Halderman, Seth D. Schoen, Nadia Heninger, William Clarkson, William Paul, Joseph A. Calandrino, Ariel J. Feldman, Jacob Appelbaum, and Edward W. Felten. Lest we remember: Cold boot attacks on encryption keys. In USENIX Security Symposium (Security'08), pages 45-60. USENIX, 2008.

[IBM] IBM. IBM Cryptocards. http://www-03.ibm.com/security/cryptocards/.

[IKNP03] Yuval Ishai, Joe Kilian, Kobbi Nissim, and Erez Petrank. Extending oblivious transfers efficiently. In Advances in Cryptology - CRYPTO'03, volume 2729 of LNCS, pages 145161. Springer, 2003.

$\left[\mathrm{IKO}^{+} 11\right]$ Yuval Ishai, Eyal Kushilevitz, Rafail Ostrovsky, Manoj Prabhakaran, and Amit Sahai. Efficient non-interactive secure computation. In Advances in Cryptology - EUROCRYPT'11, LNCS. Springer, 2011. To appear.

[Ili09] Alexander Iliev. Hardware-Assisted Secure Computation. PhD thesis, Dartmouth College, Hanover, NH, USA, 2009. http://www.cs.dartmouth.edu/ trust/Faerieplay. 
[Int03] Interational Civil Aviation Organization (ICAO). Machine Readable Travel Documents (MRTD), Doc 9303, Part 1, Fifth Edition, 2003.

[IPS08] Yuval Ishai, Manoj Prabhakaran, and Amit Sahai. Founding cryptography on oblivious transfer - efficiently. In Advances in Cryptology - CRYPTO'08, volume 5157 of LNCS, pages 572-591. Springer, 2008.

[IS05] Alexander Iliev and Sean W. Smith. More efficient secure function evaluation using tiny trusted third parties. Technical Report TR2005-551, Dartmouth College, Computer Science, Hanover, NH, July 2005.

[IS06] Alexander Iliev and Sean W. Smith. Faerieplay on tiny trusted third parties (work in progress). In Workshop on Advances in Trusted Computing (WATC'06), 2006.

[IS10] Alexander Iliev and Sean W. Smith. Small, stupid, and scalable: Secure computing with Faerieplay. In ACM Workshop on Scalable Trusted Computing (STC'10), pages 41-51. ACM, 2010.

[ISW03] Yuval Ishai, Amit Sahai, and David Wagner. Private circuits: Securing hardware against probing attacks. In Advances in Cryptology - CRYPTO'03, volume 2729 of LNCS, pages 463-481. Springer, 2003.

[JKSS10a] Kimmo Järvinen, Vladimir Kolesnikov, Ahmad-Reza Sadeghi, and Thomas Schneider. Embedded SFE: Offloading server and network using hardware tokens. In 14th International Conference on Financial Cryptography and Data Security (FC'10), volume 6052 of $L N C S$, pages 207-221. Springer, January 25-28, 2010. Full version available at http://eprint.iacr.org/2009/591

[JKSS10b] Kimmo Järvinen, Vladimir Kolesnikov, Ahmad-Reza Sadeghi, and Thomas Schneider. Efficient secure two-party computation with untrusted hardware tokens. In Ahmad-Reza Sadeghi and David Naccache, editors, Towards Hardware Intrinsic Security: Foundation and Practice, Information Security and Cryptography, pages 367-386. Springer Berlin Heidelberg, 2010.

[JKSS10c] Kimmo Järvinen, Vladimir Kolesnikov, Ahmad-Reza Sadeghi, and Thomas Schneider. Garbled circuits for leakage-resilience: Hardware implementation and evaluation of onetime programs. In 12th International Workshop on Cryptographic Hardware and Embedded Systems (CHES'10), volume 6225 of LNCS, pages 383-397. Springer, August 17-20, 2010. Full version available at http://eprint.iacr.org/2010/276.

[JP09] Ayman Jarrous and Benny Pinkas. Secure hamming distance based computation and its applications. In Applied Cryptography and Network Security (ACNS'09), volume 5536 of LNCS, pages 107-124. Springer, 2009.

[JS07] Stanislaw Jarecki and Vitaly Shmatikov. Efficient two-party secure computation on committed inputs. In Advances in Cryptology - EUROCRYPT'O7, volume 4515 of LNCS, pages 97-114. Springer, 2007.

[JSM01] Shan Jiang, Sean Smith, and Kazuhiro Minami. Securing web servers against insider attack. In Annual Computer Security Applications Conference (ACSAC'01), pages 265276. IEEE, 2001.

[Kat07] Jonathan Katz. Universally composable multi-party computation using tamper-proof hardware. In Advances in Cryptology - EUROCRYPT'O7, volume 4515 of LNCS, pages 115-128. Springer, 2007. 
[KBC97] Hugo Krawczyk, Mihir Bellare, and Ran Canetti. HMAC: Keyed-hashing for message authentication. RFC 2104 (Informational), February 1997. http://tools.ietf.org/ html/rfc2104

[KC00] Valentine Kabanets and Jin-Yi Cai. Circuit minimization problem. In ACM Symposium on Theory of Computing (STOC'00), pages 73-79. ACM, 2000.

[KJGB06] Louis Kruger, Somesh Jha, Eu-Jin Goh, and Dan Boneh. Secure function evaluation with ordered binary decision diagrams. In ACM Computer and Communications Security (CCS'06), pages 410-420. ACM, 2006.

[KJJ99] Paul C. Kocher, Joshua Jaffe, and Benjamin Jun. Differential power analysis. In Advances in Cryptology - CRYPTO'99, volume 1666 of LNCS, pages 388-397. Springer, 1999.

[KL10] Seny Kamara and Kristin Lauter. Cryptographic cloud storage. In Financial Cryptography Workshops: Real-Life Cryptographic Protocols and Standardization (RLCPS'10), volume 6054 of $L N C S$, pages 136-149. Springer, 2010.

[KM10] Jonathan Katz and Lior Malka. Private function evaluation with linear complexity. Cryptology ePrint Archive, Report 2010/528, 2010. http://eprint.iacr.org/.

[KO62] Anatolii A. Karatsuba and Yu Ofman. Multiplication of many-digital numbers by automatic computers. SSSR Academy of Sciences, 145:293-294, 1962.

[Kol05] Vladimir Kolesnikov. Gate evaluation secret sharing and secure one-round two-party computation. In Advances in Cryptology - ASIACRYPT'05, volume 3788 of LNCS, pages 136-155. Springer, 2005.

[Kol10] Vladimir Kolesnikov. Truly efficient string oblivious transfer using resettable tamperproof tokens. In Theory of Cryptography Conference (TCC'10), volume 5978 of LNCS, pages 327-342. Springer, 2010.

[KS06] Mehmet S. Kiraz and Berry Schoenmakers. A protocol issue for the malicious case of Yao's garbled circuit construction. In Symposium on Information Theory in the Benelux, pages 283-290, 2006.

[KS08a] Vladimir Kolesnikov and Thomas Schneider. Improved garbled circuit: Free XOR gates and applications. In 35th International Colloquium on Automata, Languages and Programming (ICALP'08), volume 5126 of LNCS, pages 486-498. Springer, July 6-13, 2008.

[KS08b] Vladimir Kolesnikov and Thomas Schneider. A practical universal circuit construction and secure evaluation of private functions. In 12th International Conference on Financial Cryptography and Data Security (FC'08), volume 5143 of LNCS, pages 83-97. Springer, January 28-31, 2008. Implementation available at http://thomaschneider. de/FairplayPF

[KSS09] Vladimir Kolesnikov, Ahmad-Reza Sadeghi, and Thomas Schneider. Improved garbled circuit building blocks and applications to auctions and computing minima. In 8th International Conference on Cryptology And Network Security (CANS'09), volume 5888 of $L N C S$, pages 1-20. Springer, December 12-14, 2009. Full version available at http://eprint.iacr.org/2009/411

[KSS10] Vladimir Kolesnikov, Ahmad-Reza Sadeghi, and Thomas Schneider. From dust to dawn: Practically efficient two-party secure function evaluation protocols and their modular design. Cryptology ePrint Archive, Report 2010/079, 2010. http://eprint.iacr.org/ $2010 / 079$ 
[KSWH98] John Kelsey, Bruce Schneier, David Wagner, and Chris Hall. Side channel cryptanalysis of product ciphers. In European Sumposium on Research in Computer Security (ESORICS'98), volume 1485 of LNCS, pages 97-110. Springer, 1998.

[Lem06] Kerstin Lemke. Embedded security: Physical protection against tampering attacks. In Christof Paar Kerstin Lemke and Marko Wolf, editors, Embedded Security in Cars, chapter 2, pages 207-217. Springer, 2006.

[LP07] Yehuda Lindell and Benny Pinkas. An efficient protocol for secure two-party computation in the presence of malicious adversaries. In Advances in Cryptology - EUROCRYPT'07, volume 4515 of $L N C S$, pages 52-78. Springer, 2007.

[LP09a] Yehuda Lindell and Benny Pinkas. A proof of Yao's protocol for secure two-party computation. Journal of Cryptology, 22(2):161-188, 2009. Preprint available at http: //eprint.iacr.org/2004/175

[LP09b] Yehuda Lindell and Benny Pinkas. Secure multiparty computation for privacy-preserving data mining. Journal of Privacy and Confidentiality, 1(1):59-98, 2009.

[LP10] Yehuda Lindell and Benny Pinkas. Secure two-party computation via cut-and-choose oblivious transfer. In Theory of Cryptography (TCC'11), LNCS. Springer, 2010. To appear. Preliminary version available at http://eprint.iacr.org/2010/284

[LPS08] Yehuda Lindell, Benny Pinkas, and Nigel P. Smart. Implementing two-party computation efficiently with security against malicious adversaries. In Security and Cryptography for Networks (SCN'08), volume 5229 of LNCS, pages 2-20. Springer, 2008.

$\left[\mathrm{MEK}^{+} 10\right]$ Sarah Meiklejohn, Chris Erway, Alptekin Küpçü, Theodora Hinkle, and Anna Lysyanskaya. ZKPDL: A language-based system for efficient zero-knowledge proofs and electronic cash. In USENIX Security Symposium (Security'10), pages 193-206. USENIX, 2010 .

[MiA] Microsoft SQL Azure. http://www.microsoft.com/windowsazure.

[MK10] Lior Malka and Jonathan Katz. VMCrypt - modular software architecture for scalable secure computation. Cryptology ePrint Archive, Report 2010/584, 2010. http: //eprint.iacr.org/.

[MNPS04] Dahlia Malkhi, Noam Nisan, Benny Pinkas, and Yaron Sella. Fairplay - a secure twoparty computation system. In USENIX Security Symposium (Security'04), pages 287302. USENIX, 2004. http://fairplayproject.net/fairplay.html.

[Mor] Tal Moran. The qilin crypto SDK - an open-source java SDK for rapid prototyping of cryptographic protocols. http://qilin.seas.harvard.edu.

[MOR03] Philip D. MacKenzie, Alina Oprea, and Michael K. Reiter. Automatic generation of twoparty computations. In ACM Conference on Computer and Communications Security (CCS'03), pages 210-219. ACM, 2003.

[MS08] Tal Moran and Gil Segev. David and Goliath commitments: UC computation for asymmetric parties using tamper-proof hardware. In Advances in Cryptology - EUROCRYPT'08, volume 4965 of LNCS, pages 527-544. Springer, 2008.

[NH08] Ingo Naumann and Giles Hogben. Privacy features of European eID card specifications. Network Security, 2008(8):9-13, 2008. European Network and Information Security Agency (ENISA). 
[Nie07] Jesper Buus Nielsen. Extending oblivious transfers efficiently - how to get robustness almost for free. Cryptology ePrint Archive, Report07/215, 2007. http://eprint.iacr. org/.

[Nie09] Janus D. Nielsen. Languages for Secure Multiparty Computation and Towards Strongly Typed Macros. PhD thesis, University of Aarhus, Denmark, 2009.

[NIS01] NIST, U.S. National Institute of Standards and Technology. Federal Information Processing Standards (FIPS 197). Advanced Encryption Standard (AES), November 2001. http://csrc.nist.gov/publications/fips/fips197/fips-197.pdf.

[NIS02] NIST, U.S. National Institute of Standards and Technology. Federal information processing standards (FIPS 180-2). Announcing the Secure Hash Standard, August 2002. http://csrc.nist.gov/publications/fips/fips180-2/fips-180-2.pdf

[NO09] Jesper Buus Nielsen and Claudio Orlandi. Lego for two-party secure computation. In Theory of Cryptography (TCC'09), volume 5444 of LNCS, pages 368-386. Springer, 2009.

[NP01] Moni Naor and Benny Pinkas. Efficient oblivious transfer protocols. In ACM-SIAM Symposium On Discrete Algorithms (SODA'01), pages 448-457. Society for Industrial and Applied Mathematics, 2001.

[NPS99] Moni Naor, Benny Pinkas, and Reuben Sumner. Privacy preserving auctions and mechanism design. In ACM Conference on Electronic Commerce, pages 129-139, 1999.

[NS07] Janus D. Nielsen and Michael I. Schwartzbach. A domain-specific programming language for secure multiparty computation. In Workshop on Programming Languages and Analysis for Security (PLAS'07), pages 21-30. ACM, 2007.

[NSM05] Elaine M. Newton, Latanya Sweeney, and Bradley Malin. Preserving privacy by deidentifying face images. IEEE Transactions on Knowledge and Data Engineering, $17(2): 232-243,2005$.

[NSY04] Josiane Nzouonta, Marius C. Silaghi, and Makoto Yokoo. Secure computation for combinatorial auctions and market exchanges. In Autonomous Agents and Multiagent Systems (AAMAS'04), pages 1398-1399. IEEE, 2004.

[OPJM10] Margarita Osadchy, Benny Pinkas, Ayman Jarrous, and Boaz Moskovich. SCiFI - a system for secure face identification. In IEEE Symposium on Security \& Privacy (S\&P'10), pages 239-254. IEEE, 2010.

[OST06] Dag Arne Osvik, Adi Shamir, and Eran Tromer. Cache attacks and countermeasures: The case of AES. In Cryptographers' Track at RSA Conference (CT-RSA'06), volume 3860 of $L N C S$, pages 1-20. Springer, 2006.

[Pag02] Dan Page. Theoretical use of cache memory as a cryptanalytic side-channel. Technical Report CSTR-02-003, University of Bristol, 2002.

[Pai99] Pascal Paillier. Public-key cryptosystems based on composite degree residuosity classes. In Advances in Cryptology - EUROCRYPT'99, volume 1592 of LNCS, pages 223-238. Springer, 1999.

[Ped92] Torben P. Pedersen. Non-interactive and information-theoretic secure verifiable secret sharing. In Advances in Cryptology - CRYPTO'91, volume 576 of LNCS, pages 129-140. Springer, 1992 .

[Pie09] Krzysztof Pietrzak. Provable security for physical cryptography. In Western European Workshop on Research in Cryptology (WEWoRC'09), 2009. Survey available at http: //homepages.cwi.nl/ pietrzak/publications/Pie09b.pdf. 
[PSS09] Annika Paus, Ahmad-Reza Sadeghi, and Thomas Schneider. Practical secure evaluation of semi-private functions. In 7th International Conference on Applied Cryptography and Network Security (ACNS'09), volume 5536 of LNCS, pages 89-106. Springer, June 25, 2009. Implementation available at http://www.trust.rub.de/FairplaySPF, Full version available at http://eprint.iacr.org/2009/124.

[PSSW09] Benny Pinkas, Thomas Schneider, Nigel P. Smart, and Stephen C. Williams. Secure two-party computation is practical. In 15th Advances in Cryptology - ASIACRYPT 2009, volume 5912 of $L N C S$, pages 250-267. Springer, December 6-10, 2009. Full version available at http://eprint.iacr.org/2009/314.

[Pyt] Python programming language - official website. http://www.python.org.

[QS01] Jean-Jaques Quisquater and David Samyde. Electromagnetic analysis (EMA): Measures and countermeasures for smart cards. In Research in Smart Cards (E-smart'01), volume 2140 of $L N C S$, pages 200-210. Springer, 2001.

[San09] Peter Sanders. Algorithm engineering - an attempt at a definition. In Efficient Algorithms, volume 5760 of LNCS, pages 321-340. Springer, 2009.

[Sch08] Thomas Schneider. Practical secure function evaluation. Master's thesis, University Erlangen-Nürnberg, Germany, February 27, 2008. http://thomaschneider.de/theses/ da/.

[SEC00] Standards for efficient cryptography, SEC 2: Recommended elliptic curve domain parameters. Technical report, Certicom Research, 2000. http://www.secg.org.

[Sec10] Heise Security. Hacker extracts crypto key from TPM chip, Feb 10, 2010. http://www.h-online.com/security/news/item/ Hacker-extracts-crypto-key-from-TPM-chip-927077.html.

[Sha49] Claude E. Shannon. The synthesis of two-terminal switching circuits. Bell Systems Technical Journal, 28(1):59-98, 1949.

[Sha79] Adi Shamir. How to share a secret. Communications of the ACM, 22(11):612-613, 1979.

[Sil04] Marius C. Silaghi. SMC: Secure multiparty computation language. http://cs.fit. edu/ msilaghi/SMC/, 2004.

$\left[\mathrm{SKB}^{+}\right.$09] Axel Schröpfer, Florian Kerschbaum, Debmalya Biswas, Steffen Geißinger, and Christoph Schütz. L1 - faster development and benchmarking of cryptographic protocols. In ECRYPT Workshop on Software Performance Enhancements for Encryption and Decryption and Cryptographic Compilers (SPEED-CC'O9), October 12-13, 2009.

[SKM10] Axel Schroepfer, Florian Kerschbaum, and Guenter Mueller. L1 - a programming language for mixed-protocol secure computation. Cryptology ePrint Archive, Report 2010/578, 2010. http://eprint.iacr.org/

[Sko05] Sergei P. Skorobogatov. Data remanence in flash memory devices. In Cryptographic Hardware and Embedded Systems (CHES'05), volume 3659 of LNCS, pages 339-353. Springer, 2005.

[Smi03] Sean W. Smith. Fairy dust, secrets, and the real world. IEEE Security \& Privacy, 1(1):89-93, 2003.

[SPLI06] Junhyuk Song, Radha Poovendran, Jicheol Lee, and Tetsu Iwata. The AES-CMAC Algorithm. RFC 4493 (Informational), June 2006. http://tools.ietf.org/html/rfc4493. 
$\left[\mathrm{SPY}^{+}\right.$09] Francois-Xavier Standaert, Olivier Pereira, Yu Yu, Jean-Jacques Quisquater, Moti Yung, and Elisabeth Oswald. Leakage resilient cryptography in practice. Cryptology ePrint Archive, Report 2009/341, 2009. http://eprint.iacr.org/

[SS02] Y. N. Srikant and Priti Shankar, editors. The Compiler Design Handbook: Optimizations and Machine Code Generation. CRC Press, 2002.

[SS08] Ahmad-Reza Sadeghi and Thomas Schneider. Generalized universal circuits for secure evaluation of private functions with application to data classification. In 11th International Conference on Information Security and Cryptology (ICISC'08), volume 5461 of $L N C S$, pages 336-353. Springer, December 3-5, 2008. Full version available at http://eprint.iacr.org/2008/453

[SS09] Ahmad-Reza Sadeghi and Thomas Schneider. Ask your e-doctor without telling: Privacypreserving medical diagnostics. Section Days of Ruhr-University Bochum Research School, November 6, 2009. (Poster prize awarded).

[SS10a] Ahmad-Reza Sadeghi and Thomas Schneider. Verschlüsselt Rechnen: Sichere Verarbeitung verschlüsselter medizinischer Daten am Beispiel der Klassifikation von EKGDaten. In Workshop Innovative und sichere Informationstechnologie für das Gesundheitswesen von morgen (perspeGKtive'10), volume P-174 of LNI, pages 11-25, September 8, 2010.

[SS10b] Damien Stehlé and Ron Steinfeld. Faster fully homomorphic encryption. In Advances in Cryptology - ASIACRYPT'10, volume 6477 of LNCS, pages 377-394. Springer, 2010.

[SSA ${ }^{+}$09] Marc Stevens, Alexander Sotirov, Jacob Appelbaum, Arjen K. Lenstra, David Molnar, Dag Arne Osvik, and Benne de Weger. Short chosen-prefix collisions for MD5 and the creation of a rogue CA certificate. In Advances in Cryptology - CRYPTO'09, volume 5677 of $L N C S$, pages 55-69. Springer, 2009.

[SSW08] Ahmad-Reza Sadeghi, Christian Stüble, and Marcel Winandy. Property-based TPM virtualization. In Information Security Conference (ISC'08), volume 5222 of LNCS, pages 1-16. Springer, 2008.

[SSW09] Ahmad-Reza Sadeghi, Thomas Schneider, and Immo Wehrenberg. Efficient privacypreserving face recognition. In 12th International Conference on Information Security and Cryptology (ICISC'09), volume 5984 of LNCS, pages 229-244. Springer, December 2-4, 2009. Full version available at http://eprint.iacr.org/2009/507.

[SSW10] Ahmad-Reza Sadeghi, Thomas Schneider, and Marcel Winandy. Token-based cloud computing - secure outsourcing of data and arbitrary computations with lower latency. In 3rd International Conference on Trust and Trustworthy Computing (TRUST'10) - Workshop on Trust in the Cloud, volume 6101 of LNCS, pages 417-429. Springer, June 21-23, 2010.

[ST98] Tomas Sander and Christian Tschudin. Protecting mobile agents against malicious hosts. In Mobile Agents and Security, volume 1419 of LNCS, pages 44-60. Springer, 1998.

[STM08] STMicroelectronics. Smartcard MCU with 32-bit ARM SecurCore SC300 CPU and 1.25 Mbytes high-density Flash memory. Data brief, October 2008. http://www.st.com/stonline/products/literature/bd/15066/st33f1m.pdf

[SV10] Nigel P. Smart and Fre Vercauteren. Fully homomorphic encryption with relatively small key and ciphertext sizes. In Public Key Cryptography (PKC'10), volume 6056 of LNCS, pages 420-443. Springer, 2010. 
[SW99] Sean W. Smith and Steve Weingart. Building a high-performance, programmable secure coprocessor. Computer Networks, 31(8):831-860, April 1999. Special Issue on Computer Network Security.

[SYY99] Thomas Sander, Adam Young, and Moti Yung. Non-interactive cryptocomputing for $N C^{1}$. In IEEE Symposium on Foundations of Computer Science (FOCS'99), pages 554566. IEEE, 1999.

[TAK $\left.{ }^{+} 05\right]$ Pim Tuyls, Anton H.M. Akkermans, Tom A.M. Kevenaar, Geert-Jan Schrijen, Asker M. Bazen, and Raymond N.J. Veldhuis. Practical biometric authentication with template protection. In Audio- and Video-Based Biometric Person Authentication, volume 3546 of LNCS, pages 436-446. Springer, 2005.

[TCGT09] Trusted Computing Group (TCG). TPM main specification. Main specification, Trusted Computing Group, May 2009. http://www.trustedcomputinggroup.org.

$\left[\mathrm{THH}^{+} 05\right]$ Kris Tiri, David Hwang, Alireza Hodjat, Bo-Cheng Lai, Shenglin Yang, Patrick Schaumont, and Ingrid Verbauwhede. Prototype IC with WDDL and differential routing - DPA resistance assessment. In Cryptographic Hardware and Embedded Systems (CHES'05), volume 3659 of LNCS, pages 354-365. Springer, 2005.

[TP91a] Matthew A. Turk and Alex P. Pentland. Eigenfaces for recognition. Journal of Cognitive Neuroscience, 3(1):71-86, 1991.

[TP91b] Matthew A. Turk and Alex P. Pentland. Face recognition using eigenfaces. In IEEE Computer Vision and Pattern Recognition (CVPR'91), pages 586-591. IEEE, 1991.

[TPKC07] Juan R. Troncoso-Pastoriza, Stefan Katzenbeisser, and Mehmet U. Celik. Privacy preserving error resilient DNA searching through oblivious automata. In ACM Computer and Communications Security (CCS'07), pages 519-528. ACM, 2007.

[Tur96] Brian C. H. Turton. Extending Quine-McCluskey for exclusive-or logic synthesis. IEEE Transactions on Education, 39:81-85, 1996.

[TV09] Stephen R. Tate and Roopa Vishwanathan. Improving cut-and-choose in verifiable encryption and fair exchange protocols using trusted computing technology. In Data and Applications Security (DBSec'09), volume 5645 of LNCS, pages 252-267. Springer, 2009.

[TX03a] Stephen R. Tate and Ke Xu. Mobile agent security through multi-agent cryptographic protocols. In International Conference on Internet Computing (IC'03), pages 462-470. CSREA Press, 2003.

[TX03b] Stephen R. Tate and Ke Xu. On garbled circuits and constant round secure function evaluation. Technical Report 2003-02, CoPS Labi, 2003. Technical Report 2003-02.

[Val76] Leslie G. Valiant. Universal circuits (preliminary report). In ACM Symposium on Theory of Computing (STOC'76), pages 196-203. ACM, 1976.

[Vol99] Heribert Vollmer. Introduction to Circuit Complexity: A Uniform Approach. Springer, Secaucus, NJ, USA, 1999.

[VS07] Ingrid Verbauwhede and Patrick Schaumont. Design methods for security and trust. In Design, Automation and Test in Europe (DATE'07), pages 672-677. ACM, 2007.

[Wei00] Steve H. Weingart. Physical security devices for computer subsystems: A survey of attacks and defences. In Cryptographic Hardware and Embedded Systems (CHES'00), volume 1965 of $L N C S$, pages 302-317. Springer, 2000. 
[Woe05] Philipp Woelfel. Bounds on the OBDD-size of integer multiplication via universal hashing. Journal of Computer and System Sciences, 71(4):520-534, 2005.

[WYY05] Xiaoyun Wang, Yiqun Lisa Yin, and Hongbo Yu. Finding collisions in the full SHA-1. In Advances in Cryptology - CRYPTO'05, volume 3621 of LNCS, pages 17-36. Springer, 2005.

[XT04] Ke Xu and Stephen R. Tate. Universally composable secure mobile agent computation. In Information Security Conference (ISC'04), volume 3225 of LNCS, pages 304-317. Springer, 2004.

[Yao82] Andrew C. Yao. Protocols for secure computations. In IEEE Symposium on Foundations of Computer Science (FOCS'82), pages 160-164. IEEE, 1982.

[Yao86] Andrew C. Yao. How to generate and exchange secrets. In IEEE Symposium on Foundations of Computer Science (FOCS'86), pages 162-167. IEEE, 1986.

[Yee94] Bennet S. Yee. Using Secure Coprocessors. PhD thesis, School of Computer Science, Carnegie Mellon University, May 1994. CMU-CS-94-149.

[YLP05] Yu Yu, Jussipekka Leiwo, and Benjamin Premkumar. Securely utilizing external computing power. In International Symposium on Information Technology: Coding and Computing (ITCC'05), volume 1, pages 762-767. IEEE Computer Society, 2005.

[YLP06] Yu Yu, Jussipekka Leiwo, and Benjamin Premkumar. On developing privacy-preserving compilers. International Journal of Computer Science and Network Security (IJCSNS), 6(3):154-160, March 2006. 
About the Author

\section{About the Author}

Thomas Schneider

\begin{tabular}{|c|c|}
\hline CONTACT & thomaschneider@gmail.com; http://thomaschneider.de \\
\hline PERSONAL & Born on June 1, 1983 in Koblenz, Germany. Citizen of Germany. \\
\hline EDUCATION & $\begin{array}{l}\text { Doctorate candidate in Information Sciences (eq. to Ph.D.) with dis- } \\
\text { tinction at Ruhr-University Bochum, Germany. February 2009. Advisors: } \\
\text { Prof. Dr.-Ing. Ahmad-Reza Sadeghi (Ruhr-University Bochum, Germany) } \\
\text { and Prof. Dr. Benny Pinkas (Bar Ilan University, Ramat Gan, Israel). } \\
\text { Diploma in Computer Science (Dipl.-Inf. (Univ.) eq. to M. Sc.) with } \\
\text { distinction at University Erlangen-Nürnberg, Germany. February 2008. } \\
\text { Major: computer science with specialization in theoretical computer science } \\
\text { (cryptology), hardware-software co-design, programming systems, and techni- } \\
\text { cal electronics. Minor: electrical engineering. } \\
\text { Thesis: Practical Secure Function Evaluation. Written at Alcatel-Lucent Bell } \\
\text { Labs, USA. Advisors: Dr. Vladimir Kolesnikov and Prof. Dr. Volker Strehl. } \\
\text { Intermediate Diploma in Computer Science (eq. to B.Sc.) with distinc- } \\
\text { tion at University Erlangen-Nürnberg, Germany. April 2006. } \\
\text { Thesis: Secure task migration and interprocess communication in reconfig- } \\
\text { urable, distributed, embedded systems. } \\
\text { Baccalaureate (Abitur) with distinction at Leibniz-Gymnasium Altdorf, } \\
\text { Germany. June 2002. }\end{array}$ \\
\hline $\begin{array}{l}\text { WORK } \\
\text { EXPERIENCE }\end{array}$ & $\begin{array}{l}\text { Research Assistant at Ruhr-University Bochum, System Security Lab } \\
\text { headed by Prof. Dr.-Ing. Ahmad-Reza Sadeghi, Germany (since April 2008). } \\
\text { Projects: Computer Aided Cryptography Engineering (CACE), Signal Pro- } \\
\text { cessing in the EncryptEd Domain (SPEED), European Network of Excellence } \\
\text { in Cryptology (ECRYPT) II. } \\
\text { Research Intern at Alcatel-Lucent Bell Labs, Security Solutions/ } \\
\text { Cryptographic Systems, Murray-Hill, NJ, USA with Dr. Vladimir Kolesnikov } \\
\text { (June-November 2007). } \\
\text { Teaching Assistant at University Erlangen-Nürnberg, Germany. } \\
\text { - Network Security held by Dr.-Ing. Falko Dressler (Winter 2007/08). } \\
\text { - Theoretical Computer Science 3: Complexity of Algorithms held by Prof. } \\
\text { Dr. Volker Strehl (Winter 2006/07). } \\
\text { Student Research Assistant at University Erlangen-Nürnberg, Germany, } \\
\text { Chair for Hardware-Software Co-Design headed by Prof. Dr.-Ing. Jürgen Teich } \\
\text { (June-September 2006). Project: ReCoNets. } \\
\text { Civilian Service at Information Center for Communication Assistance (ELE- } \\
\text { COK), Wichernhaus Altdorf, Germany, 2002-2003. }\end{array}$ \\
\hline HONORS & $\begin{array}{l}\text { Member of Ruhr University Research School (part of German Excel- } \\
\text { lence Initiative), 2008-2011. } \\
\text { - Scholar of German National Academic Foundation (Studienstiftung des } \\
\text { Deutschen Volkes), 2004-2008. } \\
\text { - Scholar of Bavarian Elite Program (BayBFG), 2003-2008. } \\
\text { Finalist of 21. German Informatics Contest (Bundeswettbewerb Infor- } \\
\text { matik), } 2003 \text {. }\end{array}$ \\
\hline
\end{tabular}

